\title{
Dynamic contrast-enhanced MR imaging of artherosclerotic plaque microvasculature
}

Citation for published version (APA):

van Hoof, R. H. M. (2017). Dynamic contrast-enhanced MR imaging of artherosclerotic plaque microvasculature. [Doctoral Thesis, Maastricht University]. Datawyse / Universitaire Pers Maastricht. https://doi.org/10.26481/dis.20170602rvh

Document status and date:

Published: 01/01/2017

DOI:

10.26481/dis.20170602rvh

Document Version:

Publisher's PDF, also known as Version of record

\section{Please check the document version of this publication:}

- A submitted manuscript is the version of the article upon submission and before peer-review. There can be important differences between the submitted version and the official published version of record.

People interested in the research are advised to contact the author for the final version of the publication, or visit the DOI to the publisher's website.

- The final author version and the galley proof are versions of the publication after peer review.

- The final published version features the final layout of the paper including the volume, issue and page numbers.

Link to publication

\footnotetext{
General rights rights.

- You may freely distribute the URL identifying the publication in the public portal. please follow below link for the End User Agreement:

www.umlib.nl/taverne-license

Take down policy

If you believe that this document breaches copyright please contact us at:

repository@maastrichtuniversity.nl

providing details and we will investigate your claim.
}

Copyright and moral rights for the publications made accessible in the public portal are retained by the authors and/or other copyright owners and it is a condition of accessing publications that users recognise and abide by the legal requirements associated with these

- Users may download and print one copy of any publication from the public portal for the purpose of private study or research.

- You may not further distribute the material or use it for any profit-making activity or commercial gain

If the publication is distributed under the terms of Article $25 \mathrm{fa}$ of the Dutch Copyright Act, indicated by the "Taverne" license above, 
ISBN 9789461596925

This research was performed within the framework of the Center of Translational Molecular Medicine, project PARISk (grant OIC-202), and was supported by a grant of the Dutch Heart Foundation (DHF-2008-T094).

Financial support by the Dutch Heart Foundation for the publication of this thesis is gratefully acknowledged.

Financial support for the publication of this thesis by Maastricht University, MEDIS Medical Imaging Systems BV, and Stichting Hartsvrienden RESCAR is gratefully acknowledged.

Printed by Datawyse / Universitaire Pers, Maastricht, The Netherlands

Cover design and lay-out by Cynthia Vermeulen, www.cynthiavermeulen.nl

Copyright $\odot 2017$ by Raf H.M. van Hoof. All rights reserved. No part of this publication may be reproduced, stored in a retrieval system, or transmitted in any form, or by any means without the prior written permission by the author, or when appropriate, by the publisher of the publications. 


\section{Dynamic Contrast-Enhanced MR Imaging of Atherosclerotic Plaque Microvasculature}

\section{PROEFSCHRIFT}

ter verkrijging van de graad van doctor aan de Universiteit Maastricht, op gezag van de Rector Magnificus, Prof.dr. Rianne M. Letschert volgens het besluit van het College van Decanen, in het openbaar te verdedigen op vrijdag 2 juni 2017 om 12:00 uur

door

Raf Henricus Maria van Hoof geboren op 26 oktober 1988 , te Grubbenvorst, Nederland 


\section{Promotores}

Prof.dr. J.E. Wildberger

Prof.dr. S. Heeneman

\section{Copromotor}

Dr. M.E. Kooi

\section{Beoordelingscommissie}

Prof.dr. F.M. Mottaghy (voorzitter)

Prof.dr. E. Biessen

Prof.dr. T. Leiner (UMC Utrecht)

Dr. M.E.P. Philippens (UMC Utrecht)

Prof.dr. M.J. Post 


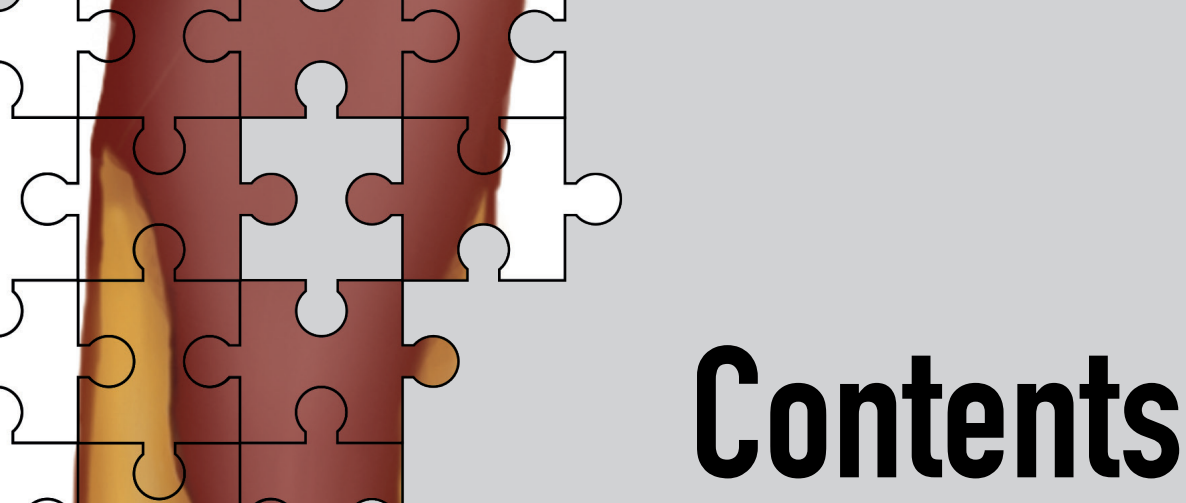

I. General Introduction 7

2. Dynamic Contrast-Enhanced MRI to Study $\quad$ I3 Atherosclerotic Plaque Microvasculature

3. Phase-Based Vascular Input Function: Improved Quantitative DCE-MRI of Atherosclerotic Plaques

4. Vessel Wall and Adventitial DCE-MRI Parameters Demonstrate Similar Correlations with Carotid Plaque Microvasculature on Histology

5. Microvasculature and Intraplaque Hemorrhage in Atherosclerotic Carotid Lesions: an MR Imaging Study

6. Combined 18-FDG PET-CT and DCE-MRI to Assess Inflammation and Microvascularization in Atherosclerotic Plaques

7. Increased Plaque Microvasculature is Associated with 91 Stroke Subtype in Symptomatic Patients Plaque: an MRI study

8. Heart Rate Lowering Treatment Leads to a Reduction 103 in Vulnerable Plaque Features in Rabbits

9. General Discussion 



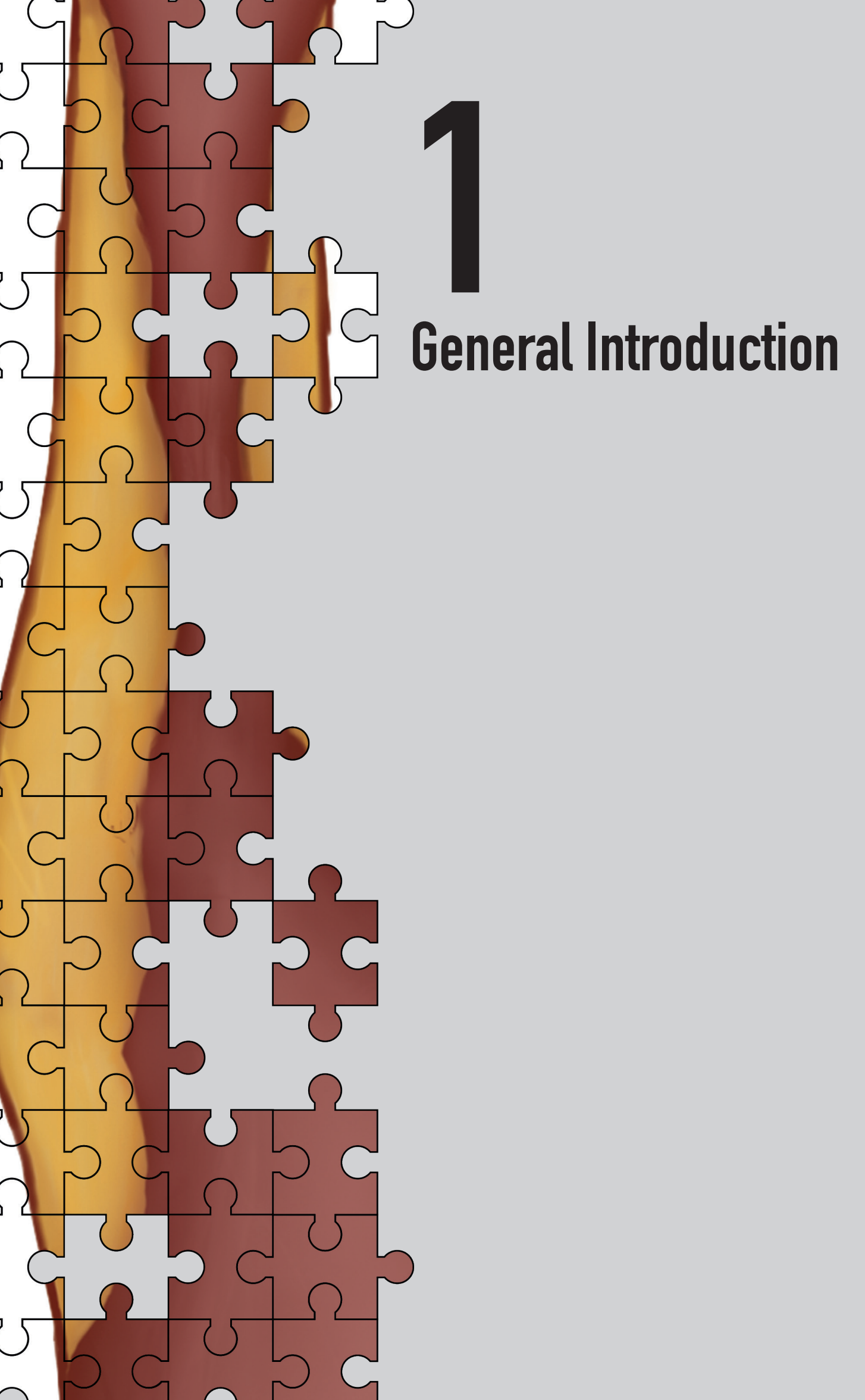


Stroke continues to be a large, worldwide health problem $(I, 2)$. Yearly, worldwide five million people die due to a stroke and an additional five million people remain permanently disabled (3). In The Netherlands, nearly 10.000 people died in 2014 due to a stroke, which accounted for $21 \%$ of all cardiovascular deaths (4). Ischemic strokes account for almost $90 \%$ of all strokes, while the other remaining $10 \%$ are hemorrhagic strokes ( 1 ). Atherosclerosis of the carotid artery accounts for 15-20\% of ischemic strokes (5), in which blood supply to (part of) the brain is (temporarily) impaired. Atherosclerosis is a systemic disease in which the arterial wall is locally thickened due to infiltration of macrophages and build up of fatty deposits and scar tissue. Treatment of patients with a carotid plaque is currently based on clinical symptoms and degree of carotid stenosis. Patients are eligible for surgical removal of the carotid artery plaque (carotid endarterectomy, CEA) when they have experienced a recent cerebrovascular ischemic event and have a carotid stenosis of $\geq 70 \%$ (6). Men with a recent ischemic event within two weeks and a carotid stenosis of $\geq 50 \%$ are also eligible for CEA (6). Large trials have shown that symptomatic patients with a 70-99\% stenosis benefit from CEA (number needed to treat $(N N T)=6$ ), however for patients with a 50-69\% stenosis the NNT increases to 22 (7). Symptomatic patients that are not eligible to undergo CEA receive best medical treatment, consisting of anti-platelet therapy and cholesterol synthesis inhibitors (6).

Histological studies on CEA specimens have shown a higher incidence of specific characteristics of atherosclerotic plaques in symptomatic patients compared to asymptomatic patients (8-12). Owing to technical improvements of non-invasive imaging modalities, the interest for these "vulnerable plaques" has increased greatly over the past decade. Vulnerable plaques are atherosclerotic plaques with an increased risk of rupture which can lead to clinical symptoms (I3). Features of these vulnerable atherosclerotic plaques are a large lipid-rich necrotic core (LRNC) with a thin fibrous cap, presence of inflammatory cells, ulcerations, and intraplaque hemorrhage (IPH) (I3), while fibrous tissue is considered a stabilizing feature of atherosclerotic lesions (14). Besides these plaque features, increased plaque microvasculature has also been suggested as an important marker of plaque vulnerability (I5). These microvessels grow from the outer layer of the vessel wall into the plaque tissue and generally have a low integrity of the endothelium (16). Therefore, they may provide an entry point for inflammatory cells and erythrocytes into the plaque tissue. Recent research has shown a weak link between increased plaque microvasculature and plaque inflammation $(17,18)$ and the presence of intraplaque hemorrhage (19). However, it was shown that the correlation between the plaque inflammation and microvasculature varied depending on clinical condition (I8). Therefore, plaque microvasculature may provide additional information compared to other vulnerable plaque features. Investigation of the plaque microvasculature may also provide further insight into pathological mechanisms involved in plaque development and destabilization.

In vivo visualization of the plaque microvasculature can be performed using contrast-enhanced ultrasound (CEUS) (20,2I), positron emission tomography (PET) (22-24), or dynamic contrast-enhanced magnetic resonance imaging (DCE-MRI) (25-3I). Due to its superior soft tissue contrast, MR imaging allows to study plaque microvasculature in relation to other plaque components (LRNC, fibrous tissue, and IPH) (32). Moreover, MRI enables the study of 
patients with a mild to moderate carotid stenosis, who rarely undergo CEA and are therefore not available for histopathological studies. Therefore, this thesis will focus on non-invasive quantification of the plaque microvasculature with DCE-MRI using semi-quantitative and quantitative parameters.

\section{THESIS HYPOTHESIS}

The hypothesis of this thesis was that non-invasive imaging of the plaque microvasculature with DCE-MRI can aid to gain further insight in the atherosclerotic process. To investigate this hypothesis, symptomatic patients with carotid atherosclerosis underwent an MRI examination and a preclinical study in atherosclerotic rabbits was conducted in order to address the following objectives:

- To further improve and validate DCE-MRI methodology

- To investigate the association between plaque microvasculature and important features of plaque vulnerability (plaque inflammation and presence of intraplaque hemorrhage)

- To explore whether plaque microvasculature is related to the type of cerebrovascular symptoms in patients with mild to moderate carotid stenosis

- To evaluate if DCE-MRI can be used as evaluation tool to investigate the effect of a heartrate reducing therapy on features of plaque vulnerability

\section{OUTLINE OF THIS THESIS}

In chapter 2 an overview of the current state of DCE-MRI to study plaque microvasculature in a preclinical and clinical setting is provided. This chapter focuses on recent literature on I) DCE-MRI methods of acquisition and image analysis of atherosclerotic plaques; 2) the association between microvasculature and other key features of plaque vulnerability (e.g. inflammation and intraplaque hemorrhage); and 3) the effects of therapeutic interventions on the plaque microvasculature measured with DCE-MRI.

Accurate determination of the contrast medium (CM) concentration within the blood plasma is essential for quantitative analysis of the plaque microvasculature with DCE-MRI. In chapter 3 , an alternative method based on the phase MRI signal to measure the CM concentration is introduced and compared to the commonly used method that is based on the magnitude of the MRI signal. Simulations and flow phantom experiments are employed to determine the effect of local blood flow velocity on magnitude and phase signal enhancement.

Some studies report DCE-MRI parameters from the adventitial region, while other studies include the entire vessel wall in DCE-MRI analysis. In chapter 4, a direct comparison between DCE-MRI parameters of these two regions is performed. Parameters are also validated with histology in symptomatic patients that were scheduled for surgical removal of carotid plaque. 
It is suggested that IPH is caused by leakage of erythrocytes from immature plaque microvessels. The exact mechanisms responsible for the development of IPH are not fully understood. The aim of the study presented in chapter 5 was to investigate in a relatively large imaging study whether there is a positive association between plaque perfusion as determined by DCE-MRI and IPH.

Increased macrophage activity may lead to hypoxia within the plaque tissue, which stimulates angiogenesis in the atherosclerotic lesion. In chapter 6, we studied whether plaque inflammation as assessed with ${ }^{18} \mathrm{~F}$-fluorodeoxyglucose $\left({ }^{18} \mathrm{~F}-\mathrm{FDG}\right)$ PET and DCE-MRI measurements of the plaque microvasculature are related.

It is known that patients who recently experienced a stroke are at increased risk for a recurrent stroke compared to patients with a recent (ocular) transient ischemic attack (TIA). The goal of the study described in chapter 7 was to explore whether carotid plaque microvasculature is associated with the type of cerebrovascular event in (ocular) TIA versus stroke patients with a mild to moderate carotid stenosis.

Deformation of the atherosclerotic lesion during the cardiac cycle during every heartbeat may play an important role in the development of vulnerable plaques. Large epidemiologic studies have shown that an elevated resting heart rate is an independent predictor of life expectancy. Chapter 8 presents the results of a preclinical rabbit study in which the effect of a heart-rate reducing agent on features of plaque vulnerability was investigated.

Chapter 9 provides a general discussion about the results of this thesis by placing them in a broader perspective and elaborates about possible future (research) goals. 


\section{REFERENCES}

I. Mozaffarian D, Benjamin EJ, Go AS, Arnett DK, Blaha MJ, Cushman M et al. Heart Disease and Stroke Statistics-2016 Update: A Report from the American Heart Association. Circulation 2015;10.116I/ CIR.0000000000000350.

2. Mozaffarian D, Benjamin EJ, Go AS, Arnett DK, Blaha MJ, Cushman M et al. Executive Summary: Heart Disease and Stroke Statistics - 2016 Update: A Report from the American Heart Association. Circulation 20 I 6; I 33:447454.

3. Mackay J, Mensah G. The Atlas of Heart Disease and Stroke - Global Burden of Stroke. 2004.

4. Van Dis I, Buddeke J, Vaartjes I, Visseren FLJ, Bots ML. Hart- En Vaatziekten in Nederland 20I5, Cijfers over Heden, Verleden En Toekomst. Hartstichting 2015.

5. Chaturvedi S, Bruno A, Feasby T, Holloway R, Benavente O, Cohen SN et al. Carotid Endarterectomy-an Evidence-Based Review: Report of the Therapeutics and Technology Assessment Subcommittee of the American Academy of Neurology. Neurology 2005;65:794-80I.

6. Neurologie NVv. Richtlijn Diagnostiek, Behandeling En Zorg Voor Patiënten Met Een Beroerte. 2008.

7. Rothwell PM, Eliasziw M, Gutnikov SA, Fox AJ, Taylor DW, Mayberg MR et al. Analysis of Pooled Data from the Randomised Controlled Trials of Endarterectomy for Symptomatic Carotid Stenosis. Lancet 2003;36I:I07-I I6.

8. Spagnoli LG, Mauriello A, Sangiorgi G, Fratoni S, Bonanno E, Schwartz RS et al. Extracranial Thrombotically Active Carotid Plaque as a Risk Factor for Ischemic Stroke. JAMA : the journal of the American Medical Association 2004;292: 1845-1852.

9. Redgrave JN, Lovett JK, Gallagher PJ, Rothwell PM. Histological Assessment of 526 Symptomatic Carotid Plaques in Relation to the Nature and Timing of Ischemic Symptoms: The Oxford Plaque Study. Circulation 2006; I 13:2320-2328.

10. Park AE, McCarthy WJ, Pearce WH, Matsumura JS, Yao JS. Carotid Plaque Morphology Correlates with Presenting Symptomatology. J Vasc Surg 1998;27:872-878; discussion 878-879.

II. Ballotta E, Da Giau G, Renon L. Carotid Plaque Gross Morphology and Clinical Presentation: A Prospective Study of 457 Carotid Artery Specimens. J Surg Res 2000;89:78-84.

12. Gao P, Chen ZQ, Bao YH, Jiao LQ, Ling F. Correlation between Carotid Intraplaque Hemorrhage and Clinical Symptoms: Systematic Review of Observational Studies. Stroke 2007;38:2382-2390.

13. Naghavi M, Libby P, Falk E, Casscells SW, Litovsky S, Rumberger J et al. From Vulnerable Plaque to Vulnerable Patient: A Call for New Definitions and Risk Assessment Strategies: Part I. Circulation 2003; I08: I664- 1672.

14. Seeger JM, Barratt E, Lawson GA, Klingman N. The Relationship between Carotid Plaque Composition, Plaque Morphology, and Neurologic Symptoms. J Surg Res 1995;58:330-336.

15. Moreno PR, Purushothaman KR, Fuster V, Echeverri D, Truszczynska H, Sharma SK et al. Plaque Neovascularization Is Increased in Ruptured Atherosclerotic Lesions of Human Aorta: Implications for Plaque Vulnerability. Circulation 2004; I I0:2032-2038.

16. Sluimer JC, Kolodgie FD, Bijnens AP, Maxfield K, Pacheco E, Kutys B et al. Thin-Walled Microvessels in Human Coronary Atherosclerotic Plaques Show Incomplete Endothelial Junctions Relevance of Compromised Structural Integrity for Intraplaque Microvascular Leakage. J Am Coll Cardiol 2009;53:I5 I7-I527.

17. Calcagno C, Ramachandran S, Izquierdo-Garcia D, Mani V, Millon A, Rosenbaum D et al. The Complementary Roles of Dynamic Contrast-Enhanced Mri and I8f-Fluorodeoxyglucose Pet/Ct for Imaging of Carotid Atherosclerosis. European journal of nuclear medicine and molecular imaging 20। 3;40: I 884- I 893.

18. Wang J, Liu H, Sun J, Xue H, Xie L, Yu S et al. Varying Correlation between I8f-Fluorodeoxyglucose Positron Emission Tomography and Dynamic Contrast-Enhanced Mri in Carotid Atherosclerosis: Implications for Plaque Inflammation. Stroke 2014;45: I842- I845.

19. Sun J, Song Y, Chen H, Kerwin WS, Hippe DS, Dong L et al. Adventitial Perfusion and Intraplaque Hemorrhage: A Dynamic Contrast-Enhanced Mri Study in the Carotid Artery. Stroke 2013;44:I03 I- 036. 
20. Staub D, Schinkel AF, Coll B, Coli S, van der Steen AF, Reed JD et al. Contrast-Enhanced Ultrasound Imaging of the Vasa Vasorum: From Early Atherosclerosis to the Identification of Unstable Plaques. JACC Cardiovasc Imaging 2010;3:761-77I.

2I. Shalhoub J, Owen DR, Gauthier T, Monaco C, Leen EL, Davies AH. The Use of Contrast Enhanced Ultrasound in Carotid Arterial Disease. Eur J Vasc Endovasc Surg 2010;39:38I-387.

22. Beer AJ, Pelisek J, Heider P, Saraste A, Reeps C, Metz S et al. Pet/Ct Imaging of Integrin Alphavbeta3 Expression in Human Carotid Atherosclerosis. JACC Cardiovasc Imaging 20 I 4;7: I 78- I87.

23. Golestani R, Zeebregts C], Terwisscha van Scheltinga AG, Lub-de Hooge MN, van Dam GM, Glaudemans AW et al. Feasibility of Vascular Endothelial Growth Factor Imaging in Human Atherosclerotic Plaque Using (89)ZrBevacizumab Positron Emission Tomography. Mol Imaging 20I3; I2:235-243.

24. Golestani R, Mirfeizi L, Zeebregts C], Westra J, de Haas HJ, Glaudemans AW et al. Feasibility of [I8f]-Rgd for Ex Vivo Imaging of Atherosclerosis in Detection of Alphavbeta3 Integrin Expression. J Nucl Cardiol 2015; $10.1007 /$ s|2350-0|4-006I-8.

25. Calcagno C, Mani V, Ramachandran S, Fayad ZA. Dynamic Contrast Enhanced (Dce) Magnetic Resonance Imaging (Mri) of Atherosclerotic Plaque Angiogenesis. Angiogenesis 2010;13:87-99.

26. Gaens ME, Backes WH, Rozel S, Lipperts M, Sanders SN, Jaspers K et al. Dynamic Contrast-Enhanced Mr Imaging of Carotid Atherosclerotic Plaque: Model Selection, Reproducibility, and Validation. Radiology 20I3;266:27I-279.

27. Kerwin W. Quantitative Magnetic Resonance Imaging Analysis of Neovasculature Volume in Carotid Atherosclerotic Plaque. Circulation 2003; 107:85I-856.

28. Kerwin WS, O'Brien KD, Ferguson MS, Polissar N, Hatsukami TS, Yuan C. Inflammation in Carotid Atherosclerotic Plaque: A Dynamic Contrast-Enhanced Mr Imaging StudyI. Radiology 2006;24I:459-468.

29. Kerwin WS, Oikawa M, Yuan C, Jarvik GP, Hatsukami TS. Mr Imaging of Adventitial Vasa Vasorum in Carotid Atherosclerosis. Magn Reson Med 2008;59:507-5।4.

30. Calcagno C, Cornily JC, Hyafil F, Rudd JHF, Briley-Saebo KC, Mani V et al. Detection of Neovessels in Atherosclerotic Plaques of Rabbits Using Dynamic Contrast Enhanced Mri and I8f-Fdg Pet. Arterioscler Thromb Vasc Biol 2008;28: I31 I- I317.

3I. Calcagno C, Vucic E, Mani V, Goldschlager G, Fayad ZA. Reproducibility of Black Blood Dynamic ContrastEnhanced Magnetic Resonance Imaging in Aortic Plaques of Atherosclerotic Rabbits. J Magn Reson Imaging 2010;32:191-198.

32. Yuan C, Mitsumori LM, Beach KW, Maravilla KR. Carotid Atherosclerotic Plaque: Noninvasive Mr Characterization and Identification of Vulnerable Lesions. Radiology 200 I;221:285-299 


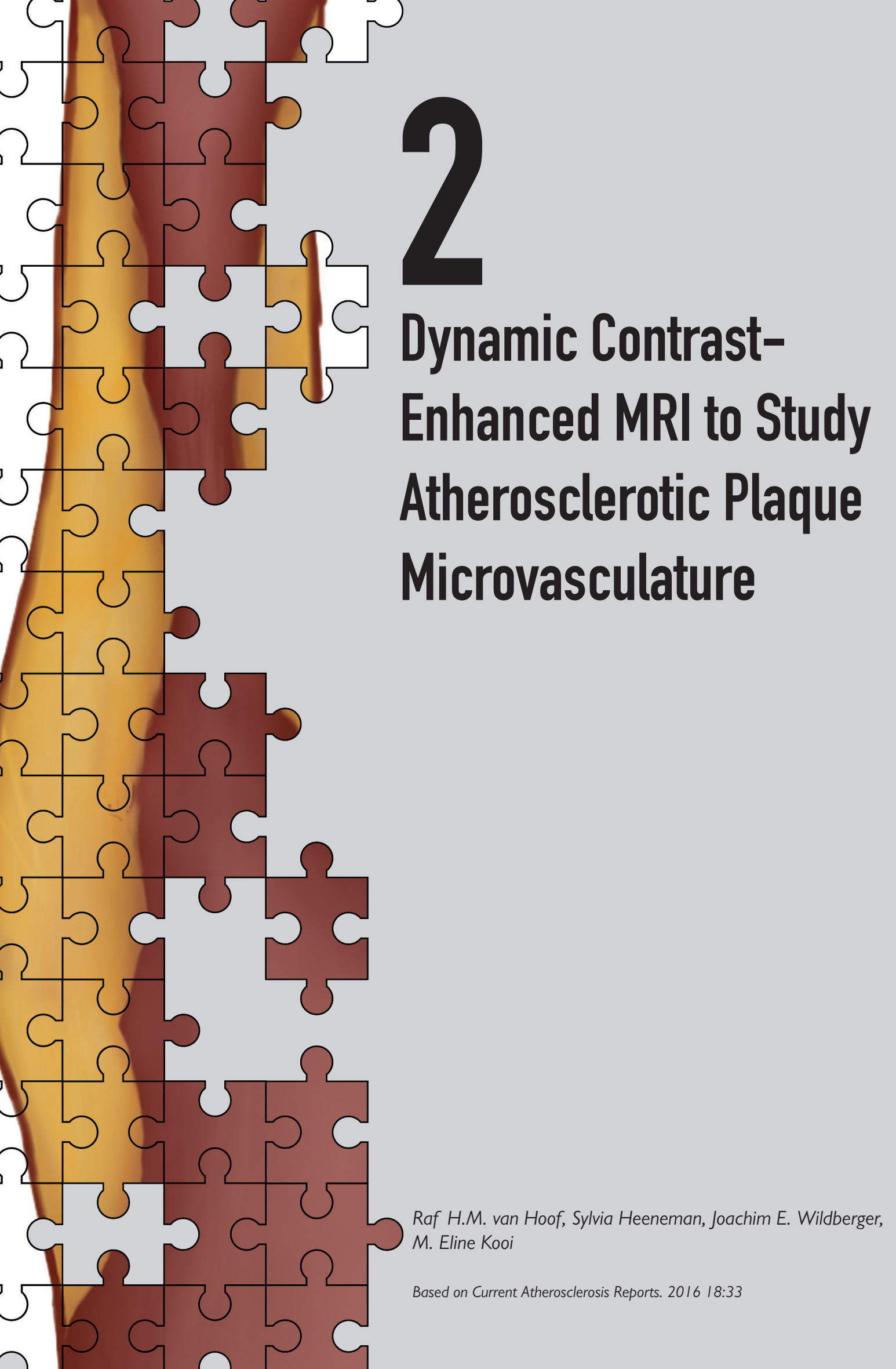




\section{Abstract}

Rupture of a vulnerable atherosclerotic plaque of the carotid artery is an important underlying cause of clinical ischemic events, such as stroke. Abundant microvasculature has been identified as an important aspect contributing to plaque vulnerability. Plaque microvasculature can be studied noninvasively with dynamic contrast-enhanced (DCE-)MRI in animals and patients. In recent years several DCE-MRI studies have been published evaluating the association between microvasculature and other key features of plaque vulnerability (e.g. inflammation and intraplaque hemorrhage), as well as the effects of novel therapeutic interventions. The present chapter reviews this literature, focusing on DCE-MRI methods of acquisition and analysis of atherosclerotic plaques, and the current state of DCE-MRI in the evaluation of plaque microvasculature in clinical and preclinical settings. 


\section{INTRODUCTION}

Rupture of a vulnerable atherosclerotic plaque is an important underlying cause of clinical ischemic events, such as stroke (I). Therefore, visualization of vulnerable plaques may aid in the identification of patients who have an increased risk for a clinical event. Inflammatory cells play an important role during the development and progression of atherosclerosis (2). Within atherosclerotic plaques, activated macrophages have a high metabolic rate, inducing hypoxia which stimulates the formation of new microvessels originating from the outer layer of the vessel wall, the adventitia $(3,4)$. These newly formed microvessels generally have impaired endothelial integrity, which can lead to extravasation of inflammatory cells and erythrocytes from the microvessel lumen into plaque tissue (4). Extravasation of erythrocytes is generally considered as an important contributing factor to intraplaque hemorrhage (IPH) (5). Because lipids constitute forty percent of the erythrocyte membrane (5), extravasation of erythrocytes leads to increased cholesterol deposition in the plaque tissue, which in turn stimulates further recruitment of inflammatory cells. All the above biological events, especially leaky plaque microvasculature, are considered key features in plaque destabilization (6).

The microvasculature in plaques are very small (up to $\sim 100 \mu \mathrm{m}$ in diameter) but can be studied non-invasively by several imaging modalities, including contrast-enhanced ultrasound (CEUS) $(7,8)$, positron emission tomography (PET) $(9-1 \mathrm{I})$, and dynamic contrast-enhanced magnetic resonance imaging (DCE-MRI) (I2). MRI is a well-established imaging modality that can be used to visualize the main plaque components: areas of $\mathrm{IPH}$, the lipid-rich necrotic core, and fibrous cap status (13-15). Early studies developed MRI for the detection of morphological and chemical components by studying specimen from surgery (carotid endarterectomy (CEA)). These ex vivo specimens were advantageous for testing and developing MRI sequences, but the lack of blood precludes studying of the dynamics from DCE $(16,17)$.

In recent years, a number of studies have applied DCE-MRI to study atherosclerotic plaque microvasculature. The present chapter reviews the current state and future potential of DCE$M R I$ in the evaluation of plaque microvasculature with applications in animals and patients. First, because the methods of DCE-MRI are now well-developed and widely applied but are not familiar to a general audience, we begin with principles and acquisition methods of DCEMRI and methods for (semi-)quantitative analysis of DCE-MRI data. Second, an overview is given of publications on DCE-MRI of plaque microvasculature (Table I) used to study one of the following aspects: I) associations between plaque microvasculature and other plaque features; 2) longitudinal changes in plaque microvasculature; 3) comparison of different animal groups and human subjects with a different cardiovascular risk profile; and 4) evaluation of therapy response. Finally, future challenges and potential for DCE-MRI to study plaque microvasculature will be discussed. 


\section{Table I: Overview of DCE-MRI studies of atherosclerotic plaque microvasculature.}

Overview of studies investigating the atherosclerotic plaque microvasculature using dynamic contrast-enhanced MRI: Subjects (human or rabbits); analysis method (quantitative or semi-quantitative); main study purpose; and study outcome are shown. Used abbreviations: contrast medium (CM), dynamic contrast-enhanced MRI (DCE-MRI), I8fluorine-fluorodeoxyglucose (I8F-FDG), positron emission tomography/computed tomography (PET-CT), area under the curve (AUC), NIRF (near-infrared fluorescence), cardiovascular disease (CVD), carotid endarterectomy (CEA), coronary heart disease (CHD), target-to-background ratio (TBR), New Zealand White (NZW).

\begin{tabular}{|c|c|c|c|}
\hline Reference & Subjects & Main Study Purpose & Main Study Outcome \\
\hline Chen et al. (I8) & $\begin{array}{l}\text { Patients with CVD } \\
\text { (AIM-HIGH Trial (19) }\end{array}$ & $\begin{array}{l}\text { Scan-rescan } \\
\text { reproducibility }\end{array}$ & $\begin{array}{l}\text { Moderate reproducibility for } \mathrm{K}^{\text {trans }} \\
\text { (Patlak) with a } 25 \% \text { coefficient of } \\
\text { variation. To limit dropout, intensive } \\
\text { operator training, optimized imaging, } \\
\text { and quality control is required. }\end{array}$ \\
\hline Kerwin et al. (20) & CEA patients & Method development & $\begin{array}{l}\text { Development of a motion } \\
\text { correcting and noise reducing } \\
\text { algorithm for the analysis of DCE- } \\
\text { MRI of carotid arteries }\end{array}$ \\
\hline Kerwin et al. (2I) & $\begin{array}{l}\text { Patients with a carotid } \\
\text { lesion } \geq \text { AHA type IV }\end{array}$ & Method comparison & $\begin{array}{l}\text { Quantitative enhancement } \\
\text { characteristics, such as } \mathrm{K}^{\text {trans }} \\
\text { (Patlak), depend on the used } \\
\text { CM (gadobenate dimeglumine vs } \\
\text { gadodiamide) }\end{array}$ \\
\hline $\begin{array}{l}\text { Ramachandran } \\
\text { et al. (22) }\end{array}$ & Humans with CVD risk & Method development & $\begin{array}{l}\text { Development of a registration } \\
\text { method for alignment of different } \\
\text { time frames of DCE-MRI of carotid } \\
\text { arteries }\end{array}$ \\
\hline Chen et al. (23) & $\begin{array}{l}\text { Humans with advanced } \\
\text { carotid disease }\end{array}$ & Method development & $\begin{array}{l}\text { Extended graphical model exhibits } \\
\text { a reduced bias in } \mathrm{K}^{\text {trans }} \text { estimation } \\
\text { compared to the Patlak model. }\end{array}$ \\
\hline Van Hoof et al. (24) & $\begin{array}{l}\text { Symptomatic patients } \\
\text { (30-99\% carotid } \\
\text { stenosis) }\end{array}$ & Method comparison & $\begin{array}{l}\text { Comparison between phase- and } \\
\text { magnitude-based vascular input } \\
\text { functions and resulting effect on } \\
\text { pharmacokinetic parameters. No } \\
\text { signal saturation due to blood } \\
\text { flow for phase-based determined } \\
\text { vascular input function }\end{array}$ \\
\hline Calcagno et al. (25) & Humans with CVD risk & Method development & $\begin{array}{l}\text { Demonstration of feasibility of } \\
\text { simultaneous VIF and vessel wall } \\
\text { imaging (extended Tofts). }\end{array}$ \\
\hline Wan et al. (26) & NZW Rabbit ${ }^{1,2}$ & Method development & $\begin{array}{l}\text { Spatio-temporal texture based } \\
\text { features (like AUC) are able to } \\
\text { distinguish between vulnerable and } \\
\text { stable plaques. }\end{array}$ \\
\hline Calcagno et al. (27) & NZW Rabbit ${ }^{3}$ & Method comparison & $\begin{array}{l}\text { Excellent reproducibility of DCE- } \\
M R I \text { derived } A \cup C \text { (interscan, } \\
\text { intraobserver, and interobserver } \\
I C C s>0.75, P<0.00 I \text { ) }\end{array}$ \\
\hline Wu et al. (28) & NZW Rabbit' & Method development & $\begin{array}{l}\text { Demonstration of feasibility of } \\
\text { simultaneous VIF and vessel wall } \\
\text { imaging with accurate estimation } \\
\text { of pharmacokinetic parameters } \\
\text { (Patlak) }\end{array}$ \\
\hline
\end{tabular}




\begin{tabular}{|c|c|c|c|}
\hline Reference & Subjects & Main Study Purpose & Main Study Outcome \\
\hline Calcagno et al. (29) & NZW Rabbit ${ }^{3}$ & Histological validation & $\begin{array}{l}\text { Positive correlation }(\rho=0.89 \\
p=0.016) \text { between AUC and } \\
\text { amount of neovessels in the intima }\end{array}$ \\
\hline Calcagno et al. (30) & NZW Rabbit ${ }^{3}$ & Histoligical validation & $\begin{array}{l}3 D \text { DCE-MRI (AUC }(\rho=0.45) \text { and } \\
K^{\text {trans }}(\text { Patlak })(\rho=0.38) \text { is able to } \\
\text { quantify microvascular permeability } \\
\text { in the entire abdominal aorta plaque }\end{array}$ \\
\hline Chen et al. (3I) & NZW Rabbit ${ }^{4}$ & $\begin{array}{l}\text { To study plaque } \\
\text { progression }\end{array}$ & $\begin{array}{l}\text { DCE-MRI (AUC) is able to } \\
\text { quantitatively assess temporal } \\
\text { changes of atherosclerotic plaques } \\
\text { over a period of three months }\end{array}$ \\
\hline Kim et al. (32) & NZW Rabbit ${ }^{3}$ & $\begin{array}{l}\text { Validation of a chip for } \\
\text { the development of } \\
\text { nanomedicines }\end{array}$ & $\begin{array}{l}\text { Increased AUC for atherosclerotic } \\
\text { animals compared to control } \\
\text { animals. Lipid-polymer hybrid } \\
\text { nanoparticle translocation is } \\
\text { correlated with AUC }(\rho=0.79 \text {, } \\
p<0.000 \text { I) }\end{array}$ \\
\hline Lobatto et al. (33) & NZW Rabbit ${ }^{3}$ & $\begin{array}{l}\text { Evaluation of } \\
\text { glucocorticoid } \\
\text { treatment for } \\
\text { atherosclerosis }\end{array}$ & $\begin{array}{l}\text { DCE-MRI (AUC) reveals early } \\
\text { changes in plaque microvascular } \\
\text { permeability after liposomal } \\
\text { glucocorticoid treatment }\end{array}$ \\
\hline Vucic et al. (34) & NZW Rabbit ${ }^{3}$ & $\begin{array}{l}\text { Evaluation of } \\
\text { pioglitazone treatment } \\
\text { for atherosclerosis }\end{array}$ & $\begin{array}{l}\text { DCE-MRI (AUC) can demonstrate } \\
\text { the anti-inflammatory effect of } \\
\text { pioglizatone on atherosclerotic } \\
\text { plaques }\end{array}$ \\
\hline Vucic et al. (35) & NZW Rabbit ${ }^{3}$ & $\begin{array}{l}\text { Evaluation of } L X R \\
\text { agonist R2 I I } 945 \\
\text { treatment for } \\
\text { atherosclerosis }\end{array}$ & $\begin{array}{l}\text { DCE-MRI (AUC) showed a } \\
\text { trend towards a decreased } \\
\text { microvasculature after treatment } \\
\text { with atorvastatin }\end{array}$ \\
\hline Chen et al. (36) & $\begin{array}{l}\text { Patients with }>50 \% \\
\text { carotid stenosis }\end{array}$ & $\begin{array}{l}\text { Comparison of plaque } \\
\text { components }\end{array}$ & $\begin{array}{l}\mathrm{K}^{\text {trans }} \text { and } \mathrm{v}_{\mathrm{p}} \text { (Patlak) differed } \\
\text { significantly between plaque } \\
\text { components (lipid core, IPH, } \\
\text { calcifications, loose matrix, and } \\
\text { fibrous tissue), except between } \\
\text { calcifications and IPH. }\end{array}$ \\
\hline Calcagno et al. (37) & $\begin{array}{l}\text { Patients with } \mathrm{CHD} \text { or } \\
\mathrm{CHD} \text { risk equivalent }\end{array}$ & $\begin{array}{l}\text { Correlation with } \\
\text { I8F-FDG PET-CT }\end{array}$ & $\begin{array}{l}\text { Weak, inverse relationship between } \\
\text { inflammation }\left({ }^{18} \mathrm{~F}-\mathrm{FDG} \text { PET-CT, }\right. \\
\text { mean TBR) and plaque perfusion } \\
\text { (DCE-MRI, } \mathrm{K}^{\text {trans }} \text { (extended TK)) }\end{array}$ \\
\hline Dong et al. (38) & $\begin{array}{l}\text { Humans (carotid plaque } \\
\text { thickness } \geq 2 \mathrm{~mm} \text { ) }\end{array}$ & $\begin{array}{l}\text { Evaluation of intensive } \\
\text { lipid therapy in } \\
\text { the treatment of } \\
\text { atherosclerosis }\end{array}$ & $\begin{array}{l}\text { Intensive lipid therapy (using } \\
\text { atorvastatin, niacin, and } \\
\text { colesevelam) results in a reduction } \\
\text { in } \mathrm{K}^{\text {trans }} \text { (Patlak) after one year }\end{array}$ \\
\hline Gaens et al. (39) & $\begin{array}{l}\text { Symptomatic patients } \\
\text { (30-99\% carotid } \\
\text { stenosis) }\end{array}$ & $\begin{array}{l}\text { Pharmacokinetic model } \\
\text { comparison }\end{array}$ & $\begin{array}{l}\text { The Patlak model is the most suited } \\
\text { quantitative model for description } \\
\text { of carotid plaque microvasculature }\end{array}$ \\
\hline Kerwin et al. (40) & CEA Patients & $\begin{array}{l}\text { Validation against } \\
\text { microvascalature on } \\
\text { histology }\end{array}$ & $\begin{array}{l}\text { Strong correlation }(\rho=0.80 \text {, } \\
P<0.00 \mathrm{I} \text { ) between } \mathrm{DCE}-\mathrm{MRI} \text { and } \\
\text { histological measured fractional } \\
\text { vascular areas. }\end{array}$ \\
\hline
\end{tabular}




\begin{tabular}{|c|c|c|c|}
\hline Reference & Subjects & Main Study Purpose & Main Study Outcome \\
\hline Kerwin et al. (4I) & CEA Patients & $\begin{array}{l}\text { Validation against } \\
\text { microvasculature } \\
\text { and inflammation on } \\
\text { histology }\end{array}$ & $\begin{array}{l}K^{\text {trans }}(\text { Patlak }) \text { is a quantitative and } \\
\text { non-invasive marker of plaque } \\
\text { inflammation }(\rho=0.75, \rho<0.00 \mathrm{I}) \text { and } \\
\text { microvasculature }(\rho=0.7 \mathrm{I}, \rho<0.00 \mathrm{I})\end{array}$ \\
\hline Kerwin et al. (42) & CEA Patients & $\begin{array}{l}\text { Validation against } \\
\text { microvasculature } \\
\text { and inflammation on } \\
\text { histology }\end{array}$ & $\begin{array}{l}\text { Adventitial } \mathrm{K}^{\text {trans }} \text { (Patlak) was } \\
\text { significantly correlated with the } \\
\text { amount of microvasculature } \\
(\rho=0.4 \mathrm{I}, P=0.04) \text { and macrophages } \\
(\rho=0.49, p=0.0 \mathrm{I})\end{array}$ \\
\hline Mani et al. (43) & $\begin{array}{l}\text { Humans with and } \\
\text { without exposure to } \\
\text { particle matter }\end{array}$ & Risk stratification & $\begin{array}{l}\text { High exposure to particle matter } \\
\text { may be associated with plaque } \\
\text { neovascularization, measured with } \\
\text { DCE-MRI (AUC) }\end{array}$ \\
\hline O’Brien et al. (44) & $\begin{array}{l}\text { Patients with CVD } \\
\text { (AIM-HIGH Trial) (19) }\end{array}$ & $\begin{array}{l}\text { Association of DCE- } \\
\text { MRI with statin therapy }\end{array}$ & $\begin{array}{l}\text { Shorter duration of statin therapy } \\
\text { before occurrence of clinical event is } \\
\text { associated with increased } v_{p} \text { (Patlak) }\end{array}$ \\
\hline Sun et al. (45) & $\begin{array}{l}\text { Symptomatic patients } \\
\text { (ischemic event }<6 \mathrm{~m} \text { ) }\end{array}$ & $\begin{array}{l}\text { Correlation between } \\
\text { DCE-MRI (Ktrans) and } \\
\text { presence of IPH }\end{array}$ & $\begin{array}{l}\text { Presence of IPH was associated with } \\
\text { an increase of } 28 \% \text { of adventitial } \\
\text { Ktrans (Patlak) }\end{array}$ \\
\hline Truijman et al. (46) & $\begin{array}{l}\text { Symptomatic patients } \\
\text { (30-69\% carotid } \\
\text { stenosis) }\end{array}$ & $\begin{array}{l}\text { Correlation with } \\
{ }^{18} \text { F-FDG PET-CT }\end{array}$ & $\begin{array}{l}\text { Weak, positive relationship between } \\
\text { inflammation }\left({ }^{18} \mathrm{~F}-\mathrm{FDG} \text { PET-CT, }\right. \\
\text { TBR) and plaque perfusion (DCE- } \\
\text { MRI, Krans (Patlak)) }\end{array}$ \\
\hline Wang et al. (47) & $\begin{array}{l}\text { Human (carotid plaque } \\
\text { thickness } \geq 2 \mathrm{~mm} \text { ) }\end{array}$ & $\begin{array}{l}\text { Correlation with } \\
{ }^{18} \mathrm{~F}-\mathrm{FDG} \text { PET-CT }\end{array}$ & $\begin{array}{l}\text { Correlation between }{ }^{18} \mathrm{~F}-\mathrm{FDG} \text { PET } \\
(\mathrm{TBR}) \text { and DCE-MRI (K }{ }^{\text {trans }}, \text { Patlak) } \\
\text { measurements varied with clinical } \\
\text { conditions (symptomatic status) }\end{array}$ \\
\hline
\end{tabular}

'Atherosclerosis was induced by a balloon injury of the aorta in combination with a high cholesterol-enriched diet (1.0\%).

${ }^{2}$ Pharmacologic triggering was performed to stimulate plaque disruption

${ }^{3}$ Atherosclerosis was induced by a balloon injury of the aorta in combination with a low cholesterol enriched diet $(<1.0 \%)$ combined with palm oil.

${ }^{4}$ Atherosclerosis was induced by a balloon injury of the aorta in combination with a low cholesterol enriched diet $(<1.0 \%)$. 


\section{DCE-MRI METHODS TO STUDY PLAQUE MICROVASCULATURE}

\section{Principles of DCE-MRI}

All DCE-MRI experiments require serial acquisition of MR images acquired in a brief time interval to study atherosclerotic plaque microvasculature (Figure I). After acquisition of anatomical references (Figure IA), the first images of the series, acquired before contrast injection, are used to determine baseline signal intensity of the atherosclerotic plaque tissue. Bolus injection of a low molecular weight non-specific Gadolinium based contrast medium (CM) follows, and image acquisition is continued for several minutes. During this period, the bolus of CM will be distributed, resulting in signal enhancement of the blood vessel lumen, vessel wall due to leakage of the CM through damaged endothelial, and other tissues, such as skeletal muscle (Figure IB). In this image, the vessel lumen (circle) appears bright. A ring of enhancement in the outer (adventitial layer) part of the vessel wall (indicated by white arrows) can be clearly observed. The signal enhancement in the vessel wall depends on flow, microvascular density, the ability of the CM to leak from the microvasculature into the extravascular extracellular space, and reflux. After analysis of the DCE-MR images, parametric maps (Figure IC) of the resulting parameter can be generated, indicating local leaky plaque microvasculature. In DCEMRI studies of atherosclerosis to date, linear or cyclic Gadolinium-based contrast media have been used.

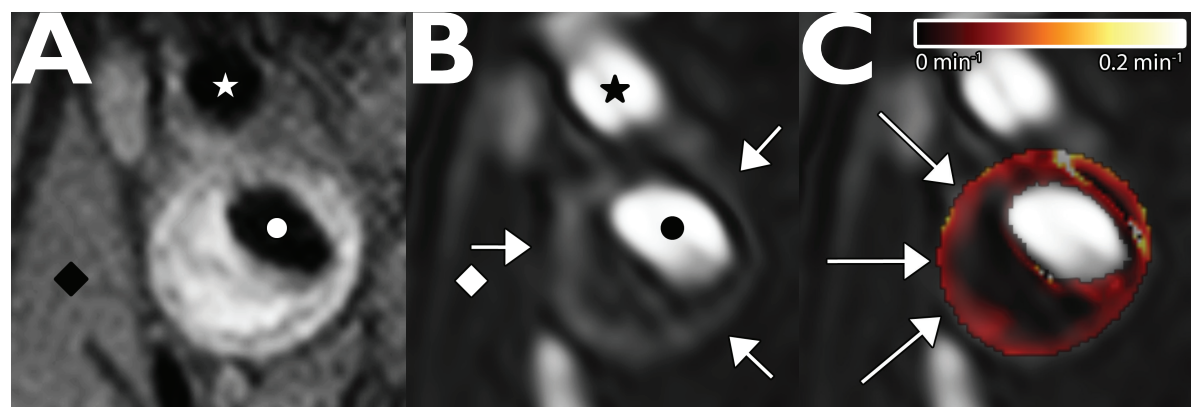

\section{Figure I}

MR images (A-C) of a transverse section of the carotid plaque in the interal carotid artery from a 64-year-old man. Figure $A$ shows a black blood $\mathrm{TI}$-weighted turbo spin echo MR image as an anatomical reference. In this image, the vessel lumen $(\bullet)$ appears in black. The atherosclerotic plaque of this patient appears hyperintense compared to the sternocleidoid muscle ( $\bullet$ ). In Figue B, a three-dimensional TI-weighted fast field-echo dynamic contrast-enhanced MR image that is acquired six minutes after contrast injection is shown. In this image, the vessel lumen $(\bullet)$ appears bright compared to the atherosclerotic plaque and surrounding tissues. A ring of enhancement can be observed at the outer part of the vessel wall (indicated by white arrows), which is attributed to the microvasculature originating from the adventitia. Finally, in Figure C, a parametric $K^{\text {trans }}$ map is overlaid on DCE-MRI image shown in Figure B. In this parametric map voxelwise determined $K^{\text {trans }}$ values are color encoded from 0 to 0.2 $\mathrm{min}^{-1}$. Within this overlay, the lipid-rich necrotic core in the center of the plaque, exhibits low Krans values (dark), while the highly vascularized adventitia (high K $K^{\text {trans }}$ values) at the outer rim (indicated by the arrows) is clearly visualized (red regions). $\bullet$, Internal carotid artery; $\star$, External carotid artery; and $\bullet$, sternocleidoid muscle. Figure adapted from Truijman et al. (46). 
DCE-MRI studies of brain and tumor perfusion mostly use a CM injection rate of $2 \mathrm{ml} / \mathrm{second}$ (typically $0.1 \mathrm{mmol} / \mathrm{kg}$ ). Such fast injection rates result in quick passage of the bolus through the vessel and a high CM peak concentration, necessitating a higher temporal resolution for $M R$ acquisition and compromising spatial resolution. For the evaluation of carotid atherosclerosis using DCE-MRI, however, high spatial resolution is required for accurate visualization of the vessel wall. Therefore, some DCE-MRI studies $(24,39)$ have used a slower injection rate of $0.5 \mathrm{ml} /$ second in plaque imaging. Previous research has shown that a high injection rate is most beneficial for high $\mathrm{K}^{\text {trans }}$ values $\left(>0.2 \mathrm{~min}^{-1}\right)$ (48). Typically, within the atherosclerotic lesion mean $\mathrm{K}^{\text {trans }}$ values below 0.15 are reported (24) and therefore a lower injection rate may be applied.

Signal enhancement-time curves of DCE-MR images can be analyzed voxelwise or using a region-of-interest. Especially in the voxelwise analysis, movement of the subject during acquisition of the different DCE-MRI sequence time frames may pose a problem. A solution is to manually shift individual time frames to correctly align the images, or alternatively, to use post-processing methods for automated movement correction and noise reduction $(20,22)$.

\section{Pulse sequences for DCE-MRI of plaque microvasculature}

Currently two main categories of pulse sequences for DCE-MRI of atherosclerotic plaque microvasculature are employed: "bright blood" or "black blood". Black blood imaging facilitates improved delineation of the inner vessel wall, whereas bright blood imaging enables to determine the CM concentration in the vessel lumen for each patient individually. Because the luminal CM concentration cannot be quantified accurately, quantitative analysis of black blood DCE-MRI with pharmacokinetic models can only be performed using a reference region model (49) or previously determined generalized input functions (50). Recently, dedicated imaging methods have been proposed combining bright and black blood images in an interleaved fashion, allowing improved delineation of the vessel wall from black blood images as well as extraction of vascular input function based on lumen signal intensity from bright blood images $(25,28)$.

A compromise between the desired spatial and the required temporal resolution must be made regardless of the imaging method used. Current studies (both in rabbits and patients) employed an in-plane spatial acquisition resolution of approximately $0.5 \times 0.5 \mathrm{~mm}^{2}$. The preclinical rabbit studies have employed a temporal resolution of 5 seconds for 2D acquisition techniques, and lower temporal resolution (30 seconds) for 3D techniques. In patient studies, the temporal resolution ranges from 15 to 30 seconds per time frame.

\section{Semi-quantitative assessment of the microvasculature}

The microvasculature can be assessed semi-quantitatively using the area-under-the-curve $(A \cup C)$ of the (relative) signal enhancement curve. This requires that start- and end time points are selected over which the AUC will be calculated. Generally, the moment of contrast arrival in the tissue of interest is chosen as the starting point, and the end time point is chosen empirically. It must be noted that when the end point is chosen relatively close to the contrast 
injection, the AUC reflects early contrast arrival, whereas a later end point will cause the AUC to reflect total leakage (and entrapment) of CM in the plaque tissue.

The main advantage of semi-quantitative analyses is the relatively easy implementation. However, the information is limited because there is no direct relationship between the AUC and (patho)physiological parameters. Although research in the field of oncology (5I) has shown that the AUC reflects pathophysiology, it does so non-specifically, meaning that one particular AUC value can indicate a number of biological properties. Thus an increased AUC can indicate increased leakage of the CM from the microvasculature, increased density of microvessels, increased flow through the microvasculature, a decrease in reflux from the extracellular extravascular space to the microvasculature, or a combination of these. Therefore, changes or differences in the AUC may result from a variety of phenomena, so that it may be difficult to attribute these changes to a single, underlying physiological cause. Similarly, effects of therapeutic interventions may potentially be obscured using the AUC. Another drawback of semi-quantitative analysis is the difficulty of direct comparison of results between studies because the AUC also depends on settings of the MR system, such as receiver gain.

\section{Validation of semi-quantitative DCE-MRI parameters}

Validation of semi-quantitative DCE-MRI was performed in several balloon injured cholesterol-fed New Zealand White rabbit studies. It was found that the AUC positively correlated with microvessel count in the intima of histological specimens (Pearson's $\rho$ of $0.89(p=0.016)$ and $0.9 \mathrm{I}(\mathrm{p}=0.0 \mathrm{I} \mathrm{I})$ for the AUC two and seven minutes after contrast injection, respectively) (29). Furthermore, later research (27) showed a good interscan and excellent intra- and inter-observer reproducibility (all ICCs $>0.75, \mathrm{p}<0.0 \mathrm{I}$ ).

Another atherosclerotic rabbit study compared two three-dimensional (3D) high spatial resolution DCE-MRI sequences (3D turbo field echo (TFE) with motion-sensitized-driven equilibrium (MSDE) preparation and a 3D turbo spin echo (TSE) sequence) (30). A moderate Pearson correlation was found between AUC and ex vivo permeability measurements using Evans Blue (an albumin binding dye used for quantification of ex vivo vascular permeability) near-infrared fluorescence (NIRF) ( $\rho=0.45$ for 3D TFE MRI and $\rho=0.39$ for 3D TSE MRI). In addition, a four-fold improvement of temporal resolution was achieved when using compressed sensing by retrospective undersampling and reconstruction. In another study, comparison between in vivo (3D DCE-MRI) and ex vivo (Cy7-labeld Near-Infrared Fluorescence [NIRF]) measures of microvascular permeability in the aortic wall of atherosclerotic rabbits showed a high degree of correlation between both imaging modalities $\left(r^{2}=0.65, p<0.0001\right)(32)$.

These studies $(27,29,30,32)$ have demonstrated reproducible representation of plaque microvasculature through semi-quantitative DCE-MRI parameters. 


\section{Quantitative assessment of the microvasculature}

Pharmacokinetic modeling

Pharmacokinetic modeling allows the quantification of CM distribution over a tissue of interest with the main advantage of deriving parameters of the in vivo physical quantities of the amount, flow and leakiness of the microvasculature.

A number of quantitative DCE-MRI data analysis models have been applied in the evaluation of atherosclerotic plaque microvasculature (Table 2). These models describe the relationship between the concentration of the (extracellular) CM in the blood plasma $\left(C_{p}\right)$ and the extracellular extravascular space $\left(\mathrm{C}_{\mathrm{e}}\right)$ according to the two-compartment model and using the parameters $K^{\text {trans }}, v_{e}$, and $v_{p}$. $K^{\text {trans }}$, the transfer constant of CM from plasma to the tissue compartment, serves as an indicator of blood supply and vessel permeability within the atherosclerotic tissue. The parameters $v_{e}$ and $v_{p}$ represent the extravascular extracellular space and the plasma fractional volume, respectively. A schematic representation of the physiological meaning of the parameters is shown in Figure 2.

\section{Table 2: Overview of quantitative DCE-MRI models used in the analysis of atherosclerosis.}

Quantitative pharmacokinetic models used for the analysis of atherosclerosis based on the two-compartment model. The modified/extended Tofts and Kermode model is the analytical solution for the two-compartment model. The extended graphical model is based on a second order Taylor expansion of the modified/extended Tofts and Kermode model.

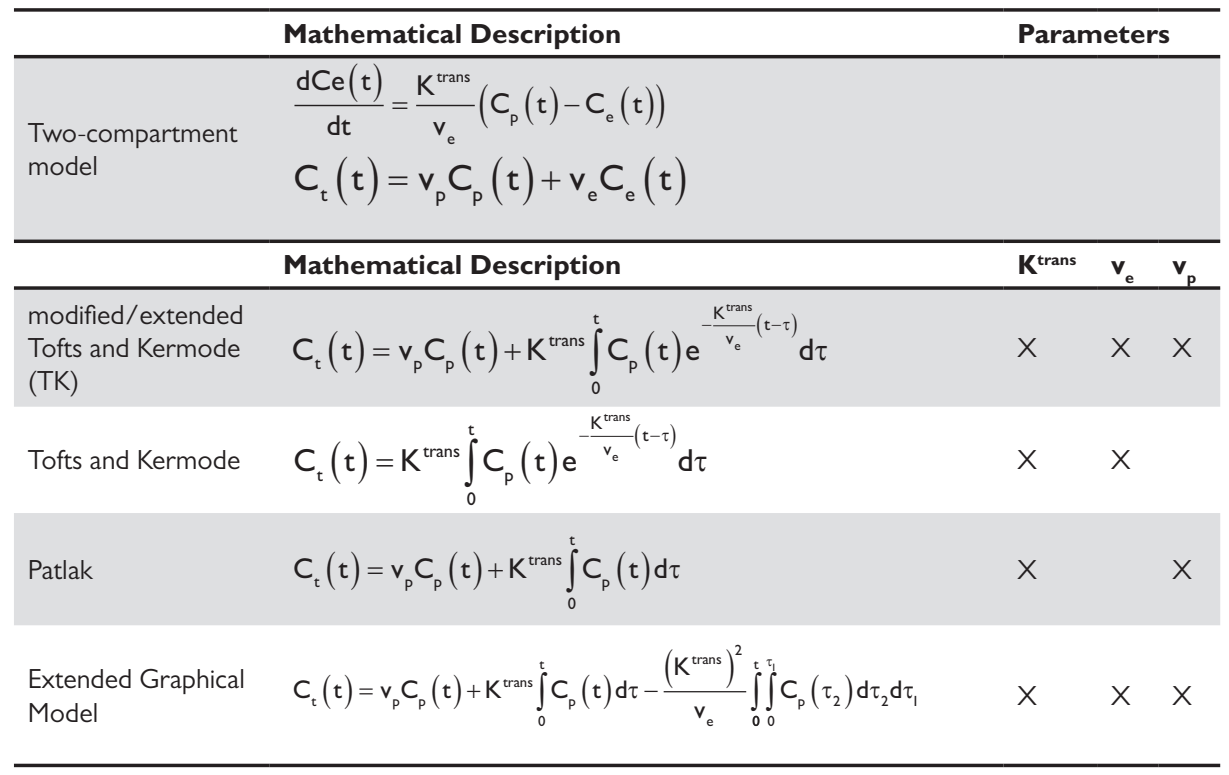




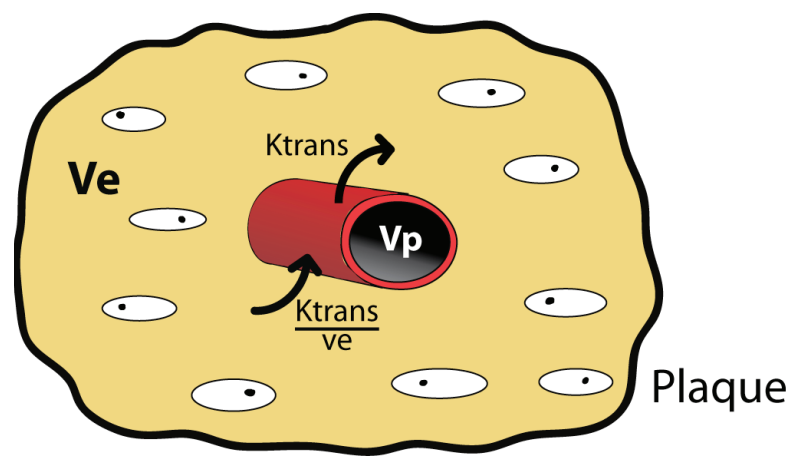

Figure 2: Schematic drawing of atherosclerotic plaque microvasculature in quantitative DCE-MRI analysis.

Schematic representation of parameters used in pharmacokinetic models for analysis of atherosclerotic plaque microvasculature. Within a single region of interest or voxel, the fractional blood volume (microvasculature) is represented by $v_{p}$, while the fraction of the extracellular extravascular space is represented by $v_{e}$. CM transfer rate from the microvasculature to the extracellular extravascular space is given by $\mathrm{K}^{\text {trans; }}$; the reflux is described by $\mathrm{K}^{\text {trans }} /$ $v_{\mathrm{e}}$. In most DCE-MRI studies an extracellular CM with a low molecular weight is used. For quantitative data analysis, therefore, a two-compartment model can be used (i.e vascular- and extracellular extra vascular compartments). Based on this general concept and setting various assumptions, several different quantitative models can be derived. An overview of these models is presented in Table 2.

The modified/extended Tofts and Kermode (TK) model $(52,53)$ is a commonly employed analytical solution for the two-compartment model $(54,55)$, estimating all three pharmacokinetic parameters $\left(K^{\text {trans }}, v_{\mathrm{e}}\right.$, and $\mathrm{v}_{\mathrm{p}}$ ). The original TK model, which was proposed for the study of multiple sclerosis (56), does not take vascular contribution into account (i.e. $v_{p}$ is assumed to be negligible). The Patlak model (57) assumes that reflux, i.e. transfer of CM from the tissue compartment back to the blood plasma $\left(\mathrm{K}^{\text {trans }} / \mathrm{v}_{\mathrm{e}}\right)$, is negligible. Recently an approximation of the modified TK model has been introduced as an intermediate solution between the modified TK and the Patlak model: the extended graphical model (23). This model uses the first-order term of a Taylor series from the modified TK model to estimate $v_{\mathrm{e}}$.

\section{Vascular Input Function}

One essential requirement for quantitative analysis of DCE-MRI data is knowledge of the CM concentration in the blood vessel over time, commonly, referred to as the arterial or vascular input function (AIF/VIF). Two main features of the VIF are a high relative peak concentration and a short bolus passage compared to other tissues. Accurate determination of the VIF requires a relatively high temporal resolution, which usually results in compromise with regard to the spatial resolution that can be achieved.

Two strategies can be employed for the determination of VIF. The first strategy is based on the assumption that VIF is similar in all subjects and a generalized population-averaged VIF, obtained from literature or determined in a cohort is used $(24,39,46,50)$. An advantage of this method is that data acquisition and analysis requirements are simplified (49). The second strategy involves measurement of patient-specific function, giving the potential advantage of accounting 
for variations between subjects (58). Previous research in oncology found comparable results using either method, and the use of population-averaged vascular input functions resulted in increased (50) or comparable (59) reproducibility. In clinical studies of atherosclerotic plaque microvasculature a generalized VIF is the most commonly chosen method, probably because of the required spatial resolution for accurate imaging of atherosclerotic plaque in the carotid artery. The generalized VIF can be obtained from a separate study cohort where acquisition is performed with a higher temporal resolution and a lower spatial resolution.

The VIF with MRI can be calculated by two different methods. The first method uses the magnitude of the acquired MR signal and is based on conversion of the relative signal enhancement to CM concentration using the Ernst equation (60). For this conversion, blood relaxation- and CM relaxivity rates are taken into account. A second method based on MR signal phase has been developed more recently $(61,62)$. First used in brain perfusion studies with dynamic susceptibility MRI (63), the technique is increasingly used in DCE-MRI $(24,64)$. Efforts have been made to compare the magnitude- and phase-based techniques $(24,59,65$ 67), showing a strong potential for the phase-based technique, allowing accurate VIF quantification. In a DCE-MRI study of 17 symptomatic patients with a mild to severe carotid stenosis, it was found that the magnitude-based VIF resulted in a strong underestimation of lumen CM concentration as compared to the phase-based VIF (24). Simulations and phantom experiments showed that this underestimation is caused by local blood flow velocity, which leads to saturation of the magnitude MR signal caused by the shortened $T$, relaxation time in the presence of CM. Analysis of $\mathrm{K}^{\text {trans }}$ values using population-averaged input functions showed a strong positive correlation between the two methods, although absolute values significantly differed.

\section{Validation of quantitative DCE-MRI parameters}

Histological validation of carotid plaque DCE-MRI has been carried out using reference specimens from patients after carotid endarterectomy (CEA). However, the drawback of all such validation studies is that these are performed in patients scheduled for CEA. Large randomized trials have shown that symptomatic patients with severe ipsilateral stenosis benefit the most from CEA (68). This population is more likely to have developed advanced atherosclerotic plaques. In addition, the surgeon removes the intima and part of the media of the vessel wall and the adventitia, from which microvasculature originates (6), is missing in the CEA specimen. An additional limitation of the comparison of in vivo MRI with histological measurements as a reference standard is the comparison of a thin histological slice to thicker MR imaging slice (typically $2 \mathrm{~mm}$ ). Due to the heterogeneous nature of atherosclerotic lesions, this may result in partial volume effects.

Despite these drawbacks, a strong and positive correlation between fractional blood volume derived from in vivo $\mathrm{MRI}$ and post surgical histology $(0.80, \mathrm{p}<0.00 \mathrm{I})$ was found in $16 \mathrm{CEA}$ patients (40). In addition, a significant Pearson correlation was reported between the transfer constants $\mathrm{K}^{\text {trans }}$, calculated from in vivo DCE-MRI with postsurgical histologic measurements of the microvessel area $\left(\rho=0.7 \mathrm{I}, \mathrm{P}<0.00 \mathrm{I}\right.$ for the entire vessel wall $K^{\text {trans }}$ and $\rho=0.4 \mathrm{I}, \mathrm{p}<0.04$ for 
adventitial $\left.\mathrm{K}^{\text {trans }}\right)$. Additionally an association between $\mathrm{K}^{\text {trans }}$ and other post surgical histological parameters was reported, i.e. macrophage density $\left(\rho=0.75, p<0.00 \mathrm{I}\right.$ for the vessel wall $K^{\text {trans }}$ and $\rho=0.49, p<0.0$ I for adventitial $\left.K^{\text {trans }}\right)$, loose matrix area $(\rho=0.50, p=0.0$ I, for vessel wall $\left.K^{\text {trans }}\right)(4 I, 42)$. It was also shown that $K^{\text {trans }}$ and $v_{p}$ differed significantly between different plaque components (lipid core, IPH, calcifications, loose matrix, and fibrous tissue), except between calcifications and IPH (36).

Reproducibility, fit error, parameter uncertainty, and correlation with histology of carotid plaque DCE-MRI was compared for four pharmacokinetic models in patients with mild to severe carotid stenosis (39). Analysis of 43 patients showed the highest relative fit error for the Tofts model, while the other three models did not differ in this regard. The Patlak model had a significant lower parameter uncertainty for $\mathrm{K}^{\text {trans }}$ as compared to the other models. Reproducibility was studied in 16 asymptomatic patients with 30-69\% carotid stenosis who underwent imaging twice with several $(4.3 \pm 2.8)$ days between the two examinations. Results showed a good reproducibility for all considered pharmacokinetic models (ICC>0.6, $\mathrm{P}<0.05$ ) for $\mathrm{K}^{\text {trans }}$, and significant scan-rescan ICCs for $v_{\mathrm{e}}$ (Tofts) and $\mathrm{v}_{\mathrm{p}}$ (Patlak). Correlation with histologic findings in 13 CEA patients showed significant positive Pearson's correlation $(\rho=0.7 ; p<0.0$ I $)$ with the entire vessel wall microvasculature for all models, with the exception of the Tofts model. It was concluded that the Patlak model was the most suited of these four models for pharmacokinetic modeling of the microvasculature in atherosclerotic plaques (39). Another study (23), however, found favorable results for the extended graphical model for simulatedand selected in vivo data of carotid plaques with good to excellent image quality. Their results showed that a compromise between noise and bias sensitivity has to be made when choosing between the Patlak and extended graphical models.

The scan-rescan reproducibility of DCE-MRI was also investigated in a multi-center study ( 8 ) of 35 subjects with established cardiovascular disease recruited from 15 hospitals. Results showed a moderate reproducibility for $\mathrm{K}^{\text {trans }}$ with a coefficient of variation of $25 \%$. The relatively high dropout rate within the study (31.4\%) suggested a need for intensive operator training, an optimized imaging protocol, and quality control.

The dependence of model parameters on CM was investigated in a study comparing two extracellular contrast media (2I). Quantitative analysis of DCE-MR images demonstrated a lower $\mathrm{K}^{\text {trans }}$ when using gadobenate dimeglumine $\left(0.0846 \mathrm{~min}^{-1}\right)$ as compared to gadodiamide $\left(0.101 \mathrm{~min}^{-1}, \mathrm{p}<0.0 \mathrm{I}\right)$, while no difference in $\mathrm{v}_{\mathrm{p}}$ was found. In order to facilitate direct comparison of quantitative DCE-MRI parameters between- or in longitudinal studies, the use of the same $C M$ is recommended.

Taken together, despite the recognized limitations, the above studies demonstrate the suitability of quantitative DCE-MRI parameters for reproducibly determining plaque microvasculature characteristics. 


\section{OVERVIEW OF DCE MRI STUDIES TO STUDY PLAQUE MICROVASCULATURE}

\section{Association between DCE-MRI parameters and other plaque features}

Many plaque characteristics and pathological features contribute to the risk for disruption and thrombosis, and studies have been designed to investigate possible associations between plaque microvasculature and other plaque features. In recent years, several studies (3I,37,45-47) were carried out to investigate associations between DCE-MRI parameters, plaque inflammation, and the presence of IPH. In a preclinical study of cholesterol-fed balloon injured atherosclerotic rabbits $(3 \mathrm{I})$, a positive Pearson correlation $(\rho=0.70, p=0.0 \mathrm{I})$ was found between DCE-MRI derived parameters and histologically determined plaque macrophage content.

The relationship between DCE-MRI parameters and plaque inflammation using ${ }^{8}$ fluorine-fluorodeoxyglucose $\left({ }^{18} \mathrm{~F}\right.$-FDG) PET-computed tomography (CT) has been investigated in several clinical studies $(37,46,47)$. One study of 49 symptomatic patients with mild to moderate carotid stenosis (46) reported a weak positive correlation (Spearman $\rho=0.30, p=0.035$ ) between plaque inflammation (mean Target-to-Background Ratio (TBR) on ${ }^{18} \mathrm{~F}-\mathrm{FDG}$ PET-CT) and plaque perfusion (mean $\mathrm{K}^{\text {trans }}$ ). Another study of 33 patients (37) with coronary heart disease (CHD) or CHD risk equivalent and a carotid plaque with TBR $\geq 1.6$ on ${ }^{18}$ F-FDG PET-CT (69), found a significant inverse relationship between plaque perfusion ( $\left.\mathrm{K}^{\text {trans }}\right)$ and plaque inflammation on ${ }^{18} \mathrm{~F}-\mathrm{FDG}$ PET-CT of $\rho=-0.24(\mathrm{p}<0.05)$. A subsequent study of $4 \mathrm{I}$ patients with carotid plaque (47) found that correlations depend on the clinical condition of patients. Overall, a weak, marginal non-significant correlation (Spearman $\rho=0.22, p=0.068$ ) was found for all, both symptomatic and asymptomatic, carotid plaques. A significant difference in Spearman correlation coefficients between TBR and $\mathrm{K}^{\text {trans }}$ was found when grouped according to the symptomatic and asymptomatic carotid plaques $(p=0.033)$ : a significant correlation (Spearman $\rho=0.59, p=0.006$ ) was found for symptomatic carotid plaques, not seen for asymptomatic plaques (Spearman $\rho=0.07, p=0.625$ ). Also, an inverse relationship was found between the time since the last neurological event and both parameters (Spearman $p=-0.94$ for TBR and Spearman $\rho=-0.69$ for $\left.\mathrm{K}^{\text {trans }}\right)$. These results point towards a complex, time-dependent interplay between inflammation and microvasculature in atherosclerotic plaques that is difficult to capture in clinical imaging.

The link between plaque microvasculature and the specific feature of IPH has been investigated in symptomatic patients with moderate to severe carotid stenosis (45). The presence of IPH on MP-RAGE MR images was associated with a significant increase in $\mathrm{K}^{\text {trans }}$ of $28 \%$ $(P<0.00 \mathrm{I})$ in the adventitial layer of the vessel wall as compared to arteries where IPH was absent $(\mathrm{p}<0.00 \mathrm{I})$. A multivariate analysis adjusting for symptomatic status, degree of stenosis and male sex, showed that the increased $\mathrm{K}^{\text {trans }}$ in arteries with IPH remained significant $(p=0.018)$. 
These studies show the potential of DCE-MRI as a tool to gain more insight in relation between plaque microvasculature and other features of vulnerable atherosclerotic lesions.

\section{Monitoring longitudinal changes in plaque microvasculature}

DCE-MRI can be used to follow progression of atherosclerotic plaques, as illustrated by a preclinical study (3I) of cholesterol-fed atherosclerotic rabbits. One group of rabbits was imaged three months after balloon denudation, immediately followed by euthanasia, and a second group at three and six months after balloon denudation. From three to six months after balloon denudation, an increase of $40 \%$ in $\mathrm{K}^{\text {trans }}$ was found measured by DCE-MRI, suggesting that DCE-MRI can be used to investigate plaque microvasculature development.

\section{Differences between different animal groups and human subjects with a different cardiovascular risk profile}

In a recent rabbit study (32), investigating the development of a microfluidic chip for potential future nanomedicines an increased AUC within the abdominal aorta for atherosclerotic animals as compared to control animals was reported. In another study of cholesterol-fed rabbits with induced plaque disruption (26), it was shown that ruptured plaques can be distinguished from stable plaques by spatial-temporal texture-based features of DCE-MRI. The effect of exposure to high particulate airborne matter on atherosclerosis was investigated in "Ground Zero" workers in New York City with high and low exposure to particulate matter using DCEMRI (43). Subjects with high exposure had a significantly higher AUC in the carotid artery $(+41 \%)$ as compared to subjects with low exposure $(p=0.016)$, indicating increased changes of the plaque microvasculature. These changes may range from increased leakage of CM from the microvasculature, increased microvessel density, increased flow through the microvasculature, decreased reflux from the extracellular extravascular space to the microvasculature, or a combination. The authors of the study concluded that a high exposure to particulate matter may lead to increased plaque microvasculature, potentially indicating an increased risk for further development of atherosclerosis.

\section{Evaluation of therapies}

DCE-MRI enables the study of plaque microvasculature changes over time, making it useful in animal and patient drug effect studies. Changes in microvasculature may reflect changes in phenotype and/or vulnerability of the atherosclerotic plaque.

DCE-MRI has been employed in several preclinical cholesterol-fed balloon injured atherosclerotic rabbit studies investigating potential anti-inflammatory treatments of atherosclerosis (3335). The effect of liposome-encapsulated prednisolone phosphate (L-PLP) on atherosclerosis was investigated using MR imaging before treatment, immediately after injection with L-PLP, and over time (33). A reduction of the plaque AUC was found from pre-treatment to two days post-treatment, revealing early changes in microvascular permeability after treatment. In a further study, the anti-inflammatory effects of pioglitazone on atherosclerotic plaques were investigated (34). DCE-MRI analysis showed a $22 \%$ decrease in AUC for the treatment 
group $(p<0.0 \mathrm{I})$ over the study time-period of three months, while no decrease in plaque enhancement was found for the control group. No changes in vessel wall area measurements were found during the study period for either animal group. A third study (35) evaluated the anti-inflammatory effects of a liver $X$ receptor $(L X R)$ agonist which induces reversal cholesterol transport, as compared to atorvastatin. The three-month treatment with $L X R$ agonist did not lead to changes of the microvasculature, whereas treatment with atorvastatin caused a trend towards a decrease in microvasculature $(p=0.06)$. No differences in vessel wall area measurements were found. Combined, these studies have shown the potential of DCE-MRI to study changes of the plaque microvasculature in the evaluation of potential new therapies. A limitation of these studies, however, is that the rabbits did not exhibit plaque disruption with luminal thrombosis, the clinical endpoint of high risk plaques.

DCE-MRI has been used to study the effect of intensive lipid therapy over a period of twelve months (38) in patients with coronary artery disease or carotid disease and increased levels $(\geq 120 \mathrm{mg} / \mathrm{dl})$ of apolipoprotein B from the Carotid Plaque Composition study (70). Results of the study show that twelve-month therapy leads to a significant reduction of $21 \%$ in $\mathrm{K}^{\text {trans. }}$. This is consistent with the hypothesis that intensive lipid therapy results in a reduction of the extent and permeability of atherosclerotic plaque microvasculature. A study with 98 subjects with established cardiovascular disease (44) selected from the AIM-HIGH trial (19) found an inverse association between $v_{p}$ (plaque microvasculature fraction) and the duration of statin therapy. Statins are commonly used to lower lipid levels and also possess anti-inflammatory properties (7I). These results suggest that a relationship exists between duration of statin therapy and plaque microvasculature, which could reflect a decreased level of vascular inflammation.

The above studies on DCE-MRI of plaque microvasculature have measured differences between treatment groups or subjects with increased cardiovascular risk, and shown that DCE$\mathrm{MRI}$ can be employed effectively as an evaluation tool.

\section{Conclusion}

Over the past decade DCE-MRI has developed from a novel imaging tool to a useful non-invasive research tool used in animal and patient studies of plaque microvasculature. DCE-MRI has been used to investigate the relationship between plaque microvasculature and other plaque features such as inflammation and intraplaque hemorrhage, for assessing effectiveness of therapeutic interventions, and in the evaluation of plaque microvasculature changes over time and between groups with increased cardiovascular risks. 


\section{Search Strategy}

The studies discussed in the present review have been identified through a database search in MEDLINE in December 2015 using the following search terms: "carotid atherosclerosis"/"atherosclerosis"/"atherosclerotic plaque"/"atherosclerotic plaques"/"plaque" AND "human"/"rabbit" AND "DCE-MRI”/“dynamic contrast enhanced MRI”/"MRI”/"dynamic contrast enhanced magnetic resonance imaging" /"magnetic resonance imaging" AND "neovessels" /"neovascularization"/"neovasculature"/"vasa vasorum"/"microvasculature"/"inflammation”. Resulting abstracts and articles were screened and references checked for possible additional studies.

\section{Acknowledgements}

The authors would like to thank Walter H. Backes and Quido G. de Lussanet for providing Figure 2 of the manuscript. 


\section{References}

I. Chaturvedi S, Bruno A, Feasby T, Holloway R, Benavente O, Cohen SN et al. Carotid Endarterectomy-an Evidence-Based Review: Report of the Therapeutics and Technology Assessment Subcommittee of the American Academy of Neurology. Neurology 2005;65:794-80 I.

2. Moore KJ, Tabas I. Macrophages in the Pathogenesis of Atherosclerosis. Cell 20I I;I45:34I-355.

3. Sluimer JC, Gasc JM, van Wanroij JL, Kisters N, Groeneweg M, Sollewijn Gelpke MD et al. Hypoxia, HypoxiaInducible Transcription Factor, and Macrophages in Human Atherosclerotic Plaques Are Correlated with Intraplaque Angiogenesis. J Am Coll Cardiol 2008;5I:I258-1265.

4. Sluimer JC, Daemen MJ. Novel Concepts in Atherogenesis: Angiogenesis and Hypoxia in Atherosclerosis. J Pathol 2009;21 8:7-29.

5. Kolodgie FD, Gold HK, Burke AP, Fowler DR, Kruth HS, Weber DK et al. Intraplaque Hemorrhage and Progression of Coronary Atheroma. N Engl J Med 2003;349:2316-2325.

6. Moreno PR, Purushothaman K-R, Sirol M, Levy AP, Fuster V. Neovascularization in Human Atherosclerosis. Circulation 2006; I I 3:2245-2252.

7. Staub D, Schinkel AF, Coll B, Coli S, van der Steen AF, Reed JD et al. Contrast-Enhanced Ultrasound Imaging of the Vasa Vasorum: From Early Atherosclerosis to the Identification of Unstable Plaques. JACC Cardiovasc Imaging 2010;3:761-77I.

8. Shalhoub J, Owen DR, Gauthier T, Monaco C, Leen EL, Davies AH. The Use of Contrast Enhanced Ultrasound in Carotid Arterial Disease. Eur J Vasc Endovasc Surg 2010;39:38I-387.

9. Beer AJ, Pelisek J, Heider P, Saraste A, Reeps C, Metz S et al. Pet/Ct Imaging of Integrin Alphavbeta3 Expression in Human Carotid Atherosclerosis. JACC Cardiovasc Imaging 20 14;7: I 78- 187.

10. Golestani R, Zeebregts C], Terwisscha van Scheltinga AG, Lub-de Hooge MN, van Dam GM, Glaudemans AW et al. Feasibility of Vascular Endothelial Growth Factor Imaging in Human Atherosclerotic Plaque Using (89)ZrBevacizumab Positron Emission Tomography. Mol Imaging 2013; 12:235-243.

II. Golestani R, Mirfeizi L, Zeebregts C], Westra J, de Haas HJ, Glaudemans AW et al. Feasibility of [18f]-Rgd for Ex Vivo Imaging of Atherosclerosis in Detection of Alphavbeta3 Integrin Expression. J Nucl Cardiol 20 I5; $10.1007 /$ s|2350-0|4-006I-8.

12. Calcagno C, Mani V, Ramachandran S, Fayad ZA. Dynamic Contrast Enhanced (Dce) Magnetic Resonance Imaging (Mri) of Atherosclerotic Plaque Angiogenesis. Angiogenesis 2010;13:87-99.

13. Yuan C, Kerwin WS, Yarnykh VL, Cai J, Saam T, Chu B et al. Mri of Atherosclerosis in Clinical Trials. NMR Biomed 2006; 19:636-654.

14. Kwee RM, van Oostenbrugge RJ, Hofstra L, Teule GJ, van Engelshoven JM, Mess WH et al. Identifying Vulnerable Carotid Plaques by Noninvasive Imaging. Neurology 2008;70:240I-2409.

15. Kwee RM, van Oostenbrugge RJ, Mess WH, Prins MH, van der Geest RJ, ter Berg JWM et al. Mri of Carotid Atherosclerosis to Identify Tia and Stroke Patients Who Are at Risk of a Recurrence. Journal of Magnetic Resonance Imaging 2012;10.1002 jmri.23918:n/a-n/a.

16. Toussaint JF, Southern JF, Fuster V, Kantor HL. T2-Weighted Contrast for Nmr Characterization of Human Atherosclerosis. Arterioscler Thromb Vasc Biol I995; I5: I533-I542.

17. Clarke SE, Hammond RR, Mitchell JR, Rutt BK. Quantitative Assessment of Carotid Plaque Composition Using Multicontrast Mri and Registered Histology. Magn Reson Med 2003;50: I I99- 1208.

18. Chen HJ, Sun J, Kerwin WS, Balu N, Neradilek MB, Hippe DS et al. Scan-Rescan Reproducibility of Quantitative Assessment of Inflammatory Carotid Atherosclerotic Plaque Using Dynamic Contrast-Enhanced 3t Cmr in a Multi-Center Study. Journal of Cardiovascular Magnetic Resonance 2014;16:51.

19. Investigators A-H, Boden WE, Probstfield JL, Anderson T, Chaitman BR, Desvignes-Nickens P et al. Niacin in Patients with Low Hdl Cholesterol Levels Receiving Intensive Statin Therapy. N Engl J Med 20 I I;365:2255-2267

20. Kerwin WS, Cai J, Yuan C. Noise and Motion Correction in Dynamic Contrast-Enhanced Mri for Analysis of Atherosclerotic Lesions. Magnetic Resonance in Medicine 2002;47:12 1 I- 1217. 
2I. Kerwin WS, Zhao X, Yuan C, Hatsukami TS, Maravilla KR, Underhill HR et al. Contrast-Enhanced Mri of Carotid Atherosclerosis: Dependence on Contrast Agent. Journal of Magnetic Resonance Imaging 2009;30:35-40.

22. Ramachandran S, Calcagno C, Mani V, Robson PM, Fayad ZA. Registration of Dynamic Contrast-Enhanced Mri of the Common Carotid Artery Using a Fixed-Frame Template-Based Squared-Difference Method. J Magn Reson Imaging 2014;39:1017.

23. Chen H, Li F, Zhao X, Yuan C, Rutt B, Kerwin WS. Extended Graphical Model for Analysis of Dynamic Contrast-Enhanced Mri. Magn Reson Med 20II;66:868-878.

24. van Hoof RH, Hermeling E, Truijman MT, van Oostenbrugge RJ, Daemen JW, van der Geest RJ et al. Phase-Based Vascular Input Function: Improved Quantitative Dce-Mri of Atherosclerotic Plaques. Med Phys 2015;42:46I9.

25. Calcagno C, Robson PM, Ramachandran S, Mani V, Kotys-Traughber M, Cham M et al. Shilo, a Novel Dual Imaging Approach for Simultaneous Hi-/Low Temporal (Low-/Hi-Spatial) Resolution Imaging for Vascular Dynamic Contrast Enhanced Cardiovascular Magnetic Resonance: Numerical Simulations and Feasibility in the Carotid Arteries. Journal of cardiovascular magnetic resonance : official journal of the Society for Cardiovascular Magnetic Resonance 2013;15:42.

26. Wan T, Madabhushi A, Phinikaridou A, Hamilton JA, Hua N, Pham T et al. Spatio-Temporal Texture (Sptet) for Distinguishing Vulnerable from Stable Atherosclerotic Plaque on Dynamic Contrast Enhancement (Dce) Mri in a Rabbit Model. Med Phys 2014;4I:-.

27. Calcagno C, Vucic E, Mani V, Goldschlager G, Fayad ZA. Reproducibility of Black Blood Dynamic ContrastEnhanced Magnetic Resonance Imaging in Aortic Plaques of Atherosclerotic Rabbits. J Magn Reson Imaging 2010;32:191-198.

28. Wu T, Wang J, Song Y, Deng X, Li A, Wei J et al. Homologous Homologous Black-Bright-Blood and Flexible Interleaved Imaging Sequence (Hobbi) for Dynamic Contrast-Enhanced Mri of the Vessel Wall. Magn Reson Med 2015;73:1754-1763.

29. Calcagno C, Cornily JC, Hyafil F, Rudd JHF, Briley-Saebo KC, Mani V et al. Detection of Neovessels in Atherosclerotic Plaques of Rabbits Using Dynamic Contrast Enhanced Mri and I8f-Fdg Pet. Arterioscler Thromb Vasc Biol 2008;28:131 I-1317.

30. Calcagno C, Lobatto ME, Dyvorne H, Robson PM, Millon A, Senders ML et al. Three-Dimensional Dynamic Contrast-Enhanced Mri for the Accurate, Extensive Quantification of Microvascular Permeability in Atherosclerotic Plaques. NMR Biomed 2015;28:1304-1314.

31. Chen H, Ricks J, Rosenfeld M, Kerwin WS. Progression of Experimental Lesions of Atherosclerosis: Assessment by Kinetic Modeling of Black-Blood Dynamic Contrast-Enhanced Mri. Magn Reson Med 2013;69:I7I2-I720.

32. Kim Y, Lobatto ME, Kawahara T, Lee Chung B, Mieszawska AJ, Sanchez-Gaytan BL et al. Probing Nanoparticle Translocation across the Permeable Endothelium in Experimental Atherosclerosis. Proc Natl Acad Sci U S A 2014; I II:1078-1083.

33. Lobatto ME, Fayad ZA, Silvera S, Vucic E, Calcagno C, Mani V et al. Multimodal Clinical Imaging to Longitudinally Assess a Nanomedical Anti-Inflammatory Treatment in Experimental Atherosclerosis. Mol Pharm 20 I0;7:20202029.

34. Vucic E, Dickson SD, Calcagno C, Rudd JH, Moshier E, Hayashi K et al. Pioglitazone Modulates Vascular Inflammation in Atherosclerotic Rabbits Noninvasive Assessment with Fdg-Pet-Ct and Dynamic ContrastEnhanced Mr Imaging. JACC Cardiovasc Imaging 20I I;4: I I00- I 109.

35. Vucic E, Calcagno C, Dickson SD, Rudd JH, Hayashi K, Bucerius J et al. Regression of Inflammation in Atherosclerosis by the Lxr Agonist R21 1945: A Noninvasive Assessment and Comparison with Atorvastatin. JACC Cardiovasc Imaging 2012;5:819-828.

36. Chen H, Cai J, Zhao X, Underhill H, Ota H, Oikawa M et al. Localized Measurement of Atherosclerotic Plaque Inflammatory Burden with Dynamic Contrast-Enhanced Mri. Magn Reson Med 2010;64:567-573. 
37. Calcagno C, Ramachandran S, Izquierdo-Garcia D, Mani V, Millon A, Rosenbaum D et al. The Complementary Roles of Dynamic Contrast-Enhanced Mri and I8f-Fluorodeoxyglucose Pet/Ct for Imaging of Carotid Atherosclerosis. European journal of nuclear medicine and molecular imaging 2013;40: I884- 1893.

38. Dong L, Kerwin WS, Chen H, Chu B, Underhill HR, Neradilek MB et al. Carotid Artery Atherosclerosis: Effect of Intensive Lipid Therapy on the Vasa Vasorum—Evaluation by Using Dynamic Contrast-Enhanced Mr Imaging. Radiology 201 I;260:224-231.

39. Gaens ME, Backes WH, Rozel S, Lipperts M, Sanders SN, Jaspers K et al. Dynamic Contrast-Enhanced Mr Imaging of Carotid Atherosclerotic Plaque: Model Selection, Reproducibility, and Validation. Radiology 20I3;266:27I-279.

40. Kerwin W, Hooker A, Spilker M, Vicini P, Ferguson M, Hatsukami T et al. Quantitative Magnetic Resonance Imaging Analysis of Neovasculature Volume in Carotid Atherosclerotic Plaque. Circulation 2003; I07:85 I-856.

4I. Kerwin WS, O’Brien KD, Ferguson MS, Polissar N, Hatsukami TS, Yuan C. Inflammation in Carotid Atherosclerotic Plaque: A Dynamic Contrast-Enhanced Mr Imaging Study I. Radiology 2006;24I:459-468.

42. Kerwin WS, Oikawa M, Yuan C, Jarvik GP, Hatsukami TS. Mr Imaging of Adventitial Vasa Vasorum in Carotid Atherosclerosis. Magn Reson Med 2008;59:507-5।4.

43. Mani V, Wong SK, Sawit ST, Calcagno C, Maceda C, Ramachandran S et al. Relationship between Particulate Matter Exposure and Atherogenic Profile in "Ground Zero" Workers as Shown by Dynamic Contrast Enhanced Mr Imaging. Int J Cardiovasc Imaging 2013;29:827-833.

44. O’Brien KD, Hippe DS, Chen H, Neradilek MB, Probstfield JL, Peck S et al. Longer Duration of Statin Therapy Is Associated with Decreased Carotid Plaque Vascularity by Magnetic Resonance Imaging. Atherosclerosis 20I5;245:74-8I.

45. Sun J, Song Y, Chen H, Kerwin WS, Hippe DS, Dong L et al. Adventitial Perfusion and Intraplaque Hemorrhage: A Dynamic Contrast-Enhanced Mri Study in the Carotid Artery. Stroke 2013;44: I03 I- 1036.

46. Truijman MT, Kwee RM, van Hoof RH, Hermeling E, van Oostenbrugge RJ, Mess WH et al. Combined I8f-Fdg Pet-Ct and Dce-Mri to Assess Inflammation and Microvascularization in Atherosclerotic Plaques. Stroke 2013;44:3568-3570.

47. Wang J, Liu H, Sun J, Xue H, Xie L, Yu S et al. Varying Correlation between I8f-Fluorodeoxyglucose Positron Emission Tomography and Dynamic Contrast-Enhanced Mri in Carotid Atherosclerosis: Implications for Plaque Inflammation. Stroke 20।4;45: I842-I845.

48. Aerts HJ, van Riel NA, Backes WH. System Identification Theory in Pharmacokinetic Modeling of Dynamic Contrast-Enhanced Mri: Influence of Contrast Injection. Magn Reson Med 2008;59: I I I I- I I I 9.

49. Yankeelov TE, Luci JJ, Lepage M, Li R, Debusk L, Lin PC et al. Quantitative Pharmacokinetic Analysis of Dce-Mri Data without an Arterial Input Function: A Reference Region Model. Magn Reson Imaging 2005;23:5I 9-529.

50. Parker GJ, Roberts C, Macdonald A, Buonaccorsi GA, Cheung S, Buckley DL et al. Experimentally-Derived Functional Form for a Population-Averaged High-Temporal-Resolution Arterial Input Function for Dynamic Contrast-Enhanced Mri. Magn Reson Med 2006;56:993-1000.

5I. Walker-Samuel S, Leach MO, Collins DJ. Evaluation of Response to Treatment Using Dce-Mri: The Relationship between Initial Area under the Gadolinium Curve (laugc) and Quantitative Pharmacokinetic Analysis. Phys Med Biol 2006;51:3593-3602.

52. Tofts PS, Brix G, Buckley DL, Evelhoch JL, Henderson E, Knopp MV et al. Estimating Kinetic Parameters from Dynamic Contrast-Enhanced T(I)-Weighted Mri of a Diffusable Tracer: Standardized Quantities and Symbols. J Magn Reson Imaging 1999; 10:223-232.

53. Tofts PS. Modeling Tracer Kinetics in Dynamic Gd-Dtpa Mr Imaging. J Magn Reson Imaging 1997;7:91-101.

54. O'Connor JP, Tofts PS, Miles KA, Parkes LM, Thompson G, Jackson A. Dynamic Contrast-Enhanced Imaging Techniques: Ct and Mri. Br J Radiol 20I I;84 Spec No 2:SI I 2-I 20. 
55. Ingrisch M, Sourbron S. Tracer-Kinetic Modeling of Dynamic Contrast-Enhanced Mri and Ct: A Primer. J Pharmacokinet Pharmacodyn 2013;40:28I-300.

56. Tofts PS, Kermode AG. Measurement of the Blood-Brain Barrier Permeability and Leakage Space Using Dynamic Mr Imaging. I. Fundamental Concepts. Magn Reson Med I99|; 17:357-367.

57. Patlak CS, Blasberg RG, Fenstermacher JD. Graphical Evaluation of Blood-to-Brain Transfer Constants from Multiple-Time Uptake Data. J Cereb Blood Flow Metab 1983;3:I-7.

58. van Osch MJ, Vonken EJ, Viergever MA, van der Grond J, Bakker CJ. Measuring the Arterial Input Function with Gradient Echo Sequences. Magn Reson Med 2003;49: I067- 1076.

59. Cron GO, Wallace JC, Stevens WD, Fortin T, Pappas BA, Wilkins RC et al. A Comparison of T2*-Weighted Magnitude and Phase Imaging for Measuring the Arterial Input Function in the Rat Aorta Following Intravenous Injection of Gadolinium Contrast Agent. Magn Reson Imaging 2005;23:619-627.

60. Schabel MC, Parker DL. Uncertainty and Bias in Contrast Concentration Measurements Using Spoiled Gradient Echo Pulse Sequences. Phys Med Biol 2008;53:2345-2373.

61. Akbudak E, Norberg RE, Conturo TE. Contrast-Agent Phase Effects: An Experimental System for Analysis of Susceptibility, Concentration, and Bolus Input Function Kinetics. Magn Reson Med 1997;38:990- 1002.

62. Akbudak E, Conturo TE. Arterial Input Functions from Mr Phase Imaging. Magn Reson Med I996;36:809-8I5.

63. Bleeker EJ, van Buchem MA, Webb AG, van Osch MJ. Phase-Based Arterial Input Function Measurements for Dynamic Susceptibility Contrast Mri. Magn Reson Med 2010;64:358-368.

64. Garpebring A, Wirestam R, Yu J, Asklund T, Karlsson M. Phase-Based Arterial Input Functions in Humans Applied to Dynamic Contrast-Enhanced Mri: Potential Usefulness and Limitations. Magma 20 I I;24:233-245.

65. Cron GO, Foottit C, Yankeelov TE, Avruch LI, Schweitzer ME, Cameron I. Arterial Input Functions Determined from Mr Signal Magnitude and Phase for Quantitative Dynamic Contrast-Enhanced Mri in the Human Pelvis. Magn Reson Med 201 I;66:498-504.

66. Korporaal JG, van den Berg CA, van Osch MJ, Groenendaal G, van Vulpen M, van der Heide UA. PhaseBased Arterial Input Function Measurements in the Femoral Arteries for Quantification of Dynamic ContrastEnhanced (Dce) Mri and Comparison with Dce-Ct. Magn Reson Med 20I I;66: I 267-I 274.

67. Simonis FF, Sbrizzi A, Beld E, Lagendijk JJ, van den Berg CA. Improving the Arterial Input Function in Dynamic Contrast Enhanced Mri by Fitting the Signal in the Complex Plane. Magn Reson Med 201 5; 10. 1002 /mrm.26023.

68. Rothwell PM, Eliasziw M, Gutnikov SA, Fox AJ, Taylor DW, Mayberg MR et al. Analysis of Pooled Data from the Randomised Controlled Trials of Endarterectomy for Symptomatic Carotid Stenosis. Lancet 2003;36I:I07-I 16.

69. Fayad ZA, Mani V, Woodward M, Kallend D, Bansilal S, Pozza J et al. Rationale and Design of Dal-Plaque: A Study Assessing Efficacy and Safety of Dalcetrapib on Progression or Regression of Atherosclerosis Using Magnetic Resonance Imaging and I8f-Fluorodeoxyglucose Positron Emission Tomography/Computed Tomography. American heart journal 201 I; I62:214-221 e212.

70. Zhao XQ, Phan BA, Chu B, Bray F, Moore AB, Polissar NL et al. Testing the Hypothesis of Atherosclerotic Plaque Lipid Depletion During Lipid Therapy by Magnetic Resonance Imaging: Study Design of Carotid Plaque Composition Study. American heart journal 2007; I54:239-246.

7I. Jain MK, Ridker PM. Anti-Inflammatory Effects of Statins: Clinical Evidence and Basic Mechanisms. Nat Rev Drug Discov 2005;4:977-987. 



\section{ABSTRACT}

\section{Purpose}

Quantitative pharmacokinetic modeling of dynamic contrast-enhanced (DCE)-MRI can be used to assess atherosclerotic plaque microvasculature, which is an important marker of plaque vulnerability. Purpose of the present study was ( $I$ ) to compare magnitude- versus phase-based vascular input functions ( $\mathrm{m}$-VIF vs ph-VIF) used in pharmacokinetic modeling and (2) to perform model calculations and flow phantom experiments to gain more insight into the differences between $\mathrm{m}$-VIF and ph-VIF.

\section{Methods}

Population averaged $\mathrm{m}$-VIF and ph-VIFs were acquired from II patients with carotid plaques and used for pharmacokinetic analysis in another 17 patients. Simulations, using the Bloch equations and the MRI scan geometry, and flow phantom experiments were performed to determine the effect of local blood velocity on the magnitude and phase signal enhancement.

\section{$\underline{\text { Results }}$}

Simulations and flow phantom experiments revealed that flow within the lumen can lead to severe underestimation of $\mathrm{m}$-VIF, while this is not the case for the ph-VIF. In line, the peak concentration of the $m-V I F$ is significantly lower than ph-VIF $(p<0.00 \mathrm{I})$, in vivo. Quantitative model parameters for $\mathrm{m}$ - and ph-VIF differed in absolute values but were moderate to strongly correlated with each other ( $K^{\text {trans }}$ Spearman's $\rho>0.93(p<0.00 I)$ and $v_{p}$ Spearman's $\rho>0.58$ $(p<0.05))$.

\section{Conclusions}

$\mathrm{m}$-VIF is strongly influenced by local blood velocity, which leads to underestimation of the contrast medium concentration. Therefore, it is advised to use ph-VIF for DCE-MRI analysis of carotid plaques for accurate quantification. 


\section{INTRODUCTION}

Rupture of an atherosclerotic plaque of the internal carotid artery is an important cause of stroke. Identification of carotid artery plaques that are prone to rupture, the so-called vulnerable plaques, is of great importance. The characteristics of a vulnerable plaque have been described in numerous histological studies (I-3); however, it remains difficult to assess plaque vulnerability in vivo. Up to now, the degree of stenosis alone is used in clinical practice, but is known to be poor in predicting vulnerability of plaques $(4,5)$. It is generally accepted that atherosclerosis is an inflammatory disease. Abundant macrophages within the inflamed plaque can lead to hypoxia due to their high rate of oxygen consumption. Hypoxia triggers the formation of new vessels from the adventitial vasa vasorum, i.e., neovascularization (6), which is considered to be a marker for plaque vulnerability (7). From these newly formed, immature, leaky vessels, it is thought that cholesterol-rich erythrocytes may enter into the plaque tissue and contribute to further destabilization of the plaque (8). MRI has emerged as a very promising imaging modality to assess plaque vulnerability in patients with carotid plaque. In recent years, an increasing number of studies assessed the microvasculature in atherosclerotic plaques using dynamic contrast-enhanced magnetic resonance imaging (DCE-MRI) (9-I5). In DCE-MRI, a series of magnetic resonance (MR) images is acquired before and after injection of a bolus of a gadolinium (Gd)-based contrast medium (CM). The CM will extravasate from the vasa vasorum into the extravascular extracellular space, resulting in enhancement of the plaque. Pharmacokinetic modeling can be used to extract parameters from the contrast-enhanced time-curve.

A variety of pharmacokinetic models exist, which are based on different underlying assumptions (16-18). Previous research has shown that the pharmacokinetic model parameters correlate with histological measures of carotid plaques, including microvessel density $(1|| 2,, \mid 5)$. A comparison of four known pharmacokinetic models (Patlak, Tofts, extended Tofts, and extended graphical model) for their ability to describe dynamic contrast medium-enhanced MR imaging of carotid atherosclerotic plaques, their scan-rescan reproducibility, and their correlation between model parameters and microvessel content on histological slices, showed that the Patlak model is most suited for describing carotid plaque enhancement (I5).

The common way to assess pharmacokinetic model parameters is by measurement of the CM concentration-time curve, both in the tissue of interest and in blood plasma. The latter is known as the vascular input function (VIF). For carotid plaque imaging, the VIF is either usually measured in the carotid artery (CA) or the jugular vein (JV). When the VIF is measured in an artery, it is also referred to as arterial input function. Obtaining a VIF from the jugular vein has the advantage that the pulsatility of the blood flow is negligible. In previous DCE-MRI studies of carotid plaques $(12,13)$, the VIF was determined using the magnitude of the acquired MR signal ( $m-V I F)$. Drawbacks of this method are (I) the nonlinear relation between the CM concentration and MRI signal enhancement and (2) the fact that flow may influence the accuracy of the determined CM concentration $(19,20)$. In recent years, the phase of the MR signal is emerging more and more into a promising alternative to measure the Gd-concentration in the vessel (ph-VIF). After initial phantom studies $(21,22)$, ph-VIFs were first used for brain perfusion studies with dynamic susceptibility MRI (23). Recently, the usability of ph-VIF in 
DCE-MRI has been demonstrated (24). The applicability of ph-VIF for the evaluation of the microvasculature in carotid plaques with its additional challenges due to the small dimensions of the carotid lesion has not been studied. The benefits of phase-based determination of the CM concentration include ( $\mathrm{I}$ ) a linear relationship between the CM concentration and phase difference $(22,25)$, (2) a favorable signal-to-noise ratio (SNR) compared to m-VIFs (20) and (3) no dependency on flow (19). There has been quite some research into the accuracy and reliability $(24,26-28)$ of phase-based techniques, focusing on partial volume effects and their corrections $(26,27)$, as well as a comparison between the magnitude and phase-based determined VIFs (29-3I).

Direct in vivo comparison between VIFs determined from both the magnitude and the phase of the MR signal has not been investigated extensively until recently. The main focus of previous research (29) has been on the reproducibility of the determined pharmacokinetic parameters. However, a detailed analysis of the influence of flow-velocity on CM concentration determined using the magnitude- and phase-based method in vivo is lacking. This comparison is, however, of great interest, since either $\mathrm{m}$-VIF or ph-VIF curves can be used for pharmacokinetic analysis. The choice may influence the resulting pharmacokinetic parameter values and their association with histological measures and clinical risk factors. For the future establishment of appropriate risk thresholds, it is essential that parameter values can be compared across centers and between studies, with minimal influence of the VIF.

The purpose of the present study was (I) to compare the m-VIF and ph-VIF from the internal jugular vein, (2) to compare ph-VIF from the internal jugular vein and common carotid artery, (3) to gain more insight into the differences between m-VIF and ph-VIF using simulations, flow phantom experiments, and in vivo imaging DCE-MRI data of patients with carotid plaques, and (4) to investigate the influence of different VIFs on quantitative DCE-MRI model parameters in carotid plaques. 


\section{Material ANd Methods}

The present study was performed within the scope of the Plaque At RISk (PARISk) study (32) and approval of the local Institutional Review Board was obtained. MRI experiments were performed on a $3 \mathrm{~T}$ whole body MRI system (Achieva, Philips Healthcare, Best, The Netherlands) using a dedicated eight-channel carotid RF coil (Shanghai Chenguan Medical Technologies Co., Shanghai, China). Five MR pulse sequences were used in all patients to facilitate delineation of plaque boundaries: (I) a three-dimensional (3D) TI-weighted turbo field-echo sequence (IR-TFE), (2) a 3D time-of-flight sequence (3D TOF), (3) a multislice two-dimensional (2D) T2-weighted turbo spin echo sequence (T2w TSE), and (4) unenhanced and (5) contrast-enhanced 2D TI-weighted turbo spin-echo sequences (TIW QIR TSE), the latter performed 6 min after contrast injection. MRI scan parameters have been described in detail previously (32).

\section{Determination of the vascular input function}

\section{MR Imaging}

For the determination of the vascular input function, I I consecutive patients ( 10 men, I women; mean age \pm standard deviation, $70.1 \pm 6.0 \mathrm{yr}$ ) with a recent (less than three months) ischemic stroke/transient ischemic attack (TIA) and $<70 \%$ carotid artery stenosis, based on the NASCET criteria (33), with a plaque thickness of at least 2-3 $\mathrm{mm}$, corresponding to an ECST stenosis of $30 \%(32,34)$, were imaged in the enddiastolic phase using an ECG-gated TI-FFE sequence with the following imaging parameters: repetition time (TR)/echo time (TE) $23.2 / 3.2 \mathrm{~ms}$, flip angle (FA) $35^{\circ}$, field of view (FOV) $150 \times 130 \mathrm{~mm}$, acquisition matrix $96 \times 70$ (linear K-space sampling), reconstructed to a matrix size of $336 \times 366$ resulting in an acquired and reconstructed pixel size of $1.56 \times 1.56$ and $0.45 \times 0.45 \mathrm{~mm}$, respectively. A single transverse slice, positioned at the common carotid artery (where no stenosis was present), with a thickness of $1.5 \mathrm{~mm}$ was acquired. In addition, a spatial saturation slab with thickness of 60 $\mathrm{mm}$ was positioned at a distal position, upstream to the jugular vein, at a distance of $15 \mathrm{~mm}$ parallel to the imaging plane. A steady-state of the MR signal was maintained by incorporation of dummy excitation pulses during the MR acquisition. At the start of the tenth time frame (scan duration approximately $4 \mathrm{~s}$ per time frame), $0.1 \mathrm{mmol} / \mathrm{kg}$ of gadobutrol (Gadovist, Bayer Health-Care, Berlin, Germany) was injected with a power injector (Spectris Solaris EP, Medrad,Warrendale) at a rate of $0.5 \mathrm{ml} / \mathrm{s}$ followed by a $20 \mathrm{ml}$ saline chaser at the same rate.

\section{Manual contour selection}

Regions-of-interest within the lumen of the bilateral common carotid arteries and internal jugular veins were drawn on the 3D TOF MR images using dedicated vessel wall analysis software (VesselMASS, Leiden University Medical Center, Leiden, The Netherlands). Care was taken to avoid partial volume effects, by keeping sufficient distance from the vessel wall. All contours were transferred to the DCE-MR images. If necessary, contours were manually adjusted and shifted to correct for small patient displacements. To correct for phase drifts of the phase-based VIF, the sternocleidomastoid muscle was used as reference region. 


\section{Magnitude-based determination of Gd concentration}

For the magnitude-based determination of the CM concentration, the average relative signal enhancement ( $\Xi$ equation $(I)$ ) was determined within the entire region-of-interest and converted to $C M$ concentration $\left(c_{C M}(t)\right)$ using the Ernst equation (equation (2)) for a spoiled steady state gradient echo pulse sequence (35) and the literature values for the longitudinal $\left(R_{1,0}\right)$ and transversal $\left(R_{2,0}\right)$ relaxation rates for blood (36) and the $r_{1}$ and $r_{2}$ relaxation rates of the $\mathrm{CM}$,

$$
\Xi(\mathrm{t})=\frac{\mathrm{S}(\mathrm{t})-\mathrm{S}(0)}{\mathrm{S}(0)}
$$

with

$$
S(t)=M_{0} \frac{\sin (F A)\left(I-e^{-T R \cdot R_{1}\left(c_{C M}(t)\right)}\right) e^{-T E \cdot R_{2}\left(c_{C M}(t)\right)}}{I-e^{-T R \cdot R_{1}\left(c_{C M}(t)\right)} \cos (F A)}
$$

in which $R_{1}$ and $R_{2}$ are given by

$$
\begin{aligned}
& \mathrm{R}_{1}\left(\mathrm{c}_{C M}(\mathrm{t})\right)=\mathrm{R}_{1,0}+\mathrm{r}_{1} \cdot \mathrm{c}_{C M}(\mathrm{t}) \\
& \mathrm{R}_{2}\left(\mathrm{c}_{C M}(\mathrm{t})\right)=\mathrm{R}_{2,0}+\mathrm{r}_{2} \cdot \mathrm{c}_{C M}(\mathrm{t})
\end{aligned}
$$

\section{Phase-based determination of the Gd concentration}

As an alternative to the $\mathrm{m}$-VIF, the phase of the MR signal can be used to determine the CM concentration, according to the following equation:

$$
c_{C M}(t)=\frac{\left(\phi(t)-\phi_{0}\right)-\left(\phi_{\text {ref }}(t)-\phi_{\text {ref }, 0}\right)}{\gamma B_{0} \chi_{m}\left(\cos ^{2}(\theta)-\frac{1}{3}\right) T E}
$$

where $\phi(t)$ is the phase in the lumen, $\phi_{\text {ref }}(t)$ is the reference phase from the sternocleidomastoid muscle, $\phi_{0}$ and $\phi_{\text {ref, } 0}$ are the baseline phase before contrast injection from the lumen and a reference region, respectively, $\gamma$ is the proton gyromagnetic ratio $\left(42.58 \mathrm{MHz} \mathrm{T}^{-1}\right), \mathrm{B}_{0}$ is the main magnetic field strength, $\chi_{\mathrm{m}}$ is the molar susceptibility for $\mathrm{Gd}\left(3.4 \times 10^{-7} \mathrm{mM}^{-1}\right), \theta$ is the angle of the vessel relative to the main magnetic field, which was determined using the 3D TOF MRI images.

\section{Generalized group averaged vascular input function curves}

A group averaged vascular input function was determined using a generalized equation which was first introduced by Parker et al. (37). and slightly modified by Gaens et al. (15). The function compromises of a single Gaussian and an exponential modulated with a sigmoid function,

$$
c_{V I F}(t)=\frac{A}{\sigma \sqrt{2 \pi}} e^{-(t-T)^{2} / 2 \sigma^{2}}+\frac{\alpha \cdot e^{-\beta t}}{1+e^{-s(t-\tau)}}
$$


where A, $\sigma$, and $\mathrm{T}$ are the scaling constant, width, and center of the Gaussian, respectively; $\alpha$ and $\beta$ are the amplitude and decay constant of the exponential function, respectively; and $s$ and $\tau$ the width and center of the sigmoid, respectively. For the group averaged VIF, the individual CM concentration-time curves for these II patients from the vessels at both sides were used to construct a single fit for every method, i.e., the group averaged $\mathrm{m}$-VIF of the jugular vein and group averaged ph-VIF of the jugular vein and the carotid artery. The intersubject variation was estimated for each method as the root mean square error relative to the peak CM concentration for all the group averaged VIFs.

\section{DCE-MRI of carotid plaque}

MR Imaging

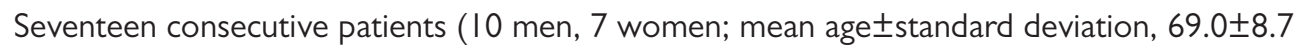
$\mathrm{yr}$ ) with a recent (less than three months) ischemic stroke/TIA and a carotid artery stenosis of $<99 \%$ (i.e., no complete occlusion) and a plaque thickness at least 2-3 mm, corresponding to an ECST stenosis of 30\% (32,34) were imaged at end-diastole using an ECG-gated 3D TI-TFE MRI pulse sequence with the following parameters: TR/TE $11.6 / 5.7 \mathrm{~ms}$, FA $35^{\circ}$, FOV I $30 \times 130$ mm, acquisition matrix 208×206 (linear K-space sampling), reconstructed to $512 \times 512$ resulting in an acquired and reconstructed in plane resolution of $0.63 \times 0.63$ and $0.25 \times 0.25 \mathrm{~mm}$, respectively, and with five consecutive slices (thickness $2 \mathrm{~mm}$ ). At the beginning of the third time frame (scan duration approximately $20 \mathrm{~s}$ per time frame), $0.1 \mathrm{mmol} /$ kg of gadobutrol (Gadovist, Bayer HealthCare, Berlin, Germany) was injected with a power injector (Spectris Solaris EP, Medrad,Warrendale) at a rate of $0.5 \mathrm{ml} / \mathrm{s}$ followed by a $20 \mathrm{ml}$ saline chaser at the same rate.

\section{Contour selection}

Contours were drawn manually for the vascular lumen, similar as described above, with the difference that the contour now contained the complete lumen. Outer plaque contours were drawn by using a combination of T2-w TSE, and pre- and postcontrast TI-w QIR TSE. Pharmacokinetic modeling was performed as previously described (I5) using the Patlak model, resulting in quantitative model parameters $\left(K^{\text {trans }}\right.$ and $\left.v_{p}\right)$. The agreement between the model parameters obtained for each of the three VIFs was analyzed by performing a Wilcoxon signed rank test and by calculating the Spearman correlation coefficient.

\section{The effect of local blood inflow on magnitude signal enhancement}

Simulations

The possible effect of flow on the CM concentration-time curve based on the magnitude images from the jugular vein is estimated as described below. In steady state, saturated spins will move from the saturation slab $(\mathrm{Mz}=0)$ to the imaging slice, and hence the longitudinal relaxation of the magnetization of the inflowing spins is dependent on their travel time according to

$$
M_{z}\left(t_{\text {blood }}, c_{C M}\right)=M_{0}\left(I-e^{-t_{\text {blood }} \cdot R_{1}\left(c_{C M}\right)}\right)
$$

In equation (7), the equilibrium magnetization is given by $M_{0}, R_{l}\left(c_{C M}\right)$ is the longitudinal 
relaxation rate of blood, which is influenced by the CM concentration $\left(\mathrm{c}_{\mathrm{CM}}\right)$, and $\mathrm{t}_{\text {blood }}$ is the travel time for the blood between the spatial saturation slab and the imaging plane, which can be determined from equation (8), in which $d_{\text {slab }}$ is the distance between the lower part of the saturation slab and the upper part of the imaging plane $(15 \mathrm{~mm})$, and $v_{\text {blood }}(r)$ is the local blood flow velocity within the vessel at position $r$, which is the (radial) distance to the axis of the blood vessel,

$$
\mathrm{t}_{\text {blood }}=\frac{\mathrm{d}_{\text {slab }}}{\mathrm{v}_{\text {blood }}(r)}
$$

At the imaging plane, the resulting longitudinal magnetization (described by equation (7)) is excitated with an excitation pulse with a FA, for which the subsequent signal relaxation is described by expressions ( 9 ) and ( 10$)$, respectively, in which $t_{n}$ is the time after the $n$-th excitation pulse $\left(0<t_{n}<T R\right)$. To take into account possible multiple excitations of the protons within the imaging slice, the maximum number of excitations $\left(\mathrm{n}_{\max }\right)$ that is experienced by the spins is determined according to equation (I I),

$$
\begin{aligned}
& M_{z}\left(t_{\text {blood }}, n, t_{n}, c_{C M}\right)=\left(M_{z}\left(t_{\text {blood }}, n-I, T R, c_{C M}\right) \cdot \cos (F A)\right) \cdot e^{-t_{n} R_{1}\left(c_{C M}\right)}+M_{0}\left(I-e^{-t_{n} \cdot R_{1}\left(c_{C M}\right)}\right)(9) \\
& M_{x y}\left(t_{\text {blood }}, n, t_{n}, c_{C M}\right)=\left(M_{z}\left(t_{\text {blood }}, n-I, T R, c_{C M}\right) \cdot \sin (F A)\right) \cdot e^{-t_{n} R_{2}\left(c_{C M}\right)} \\
& n_{\max }(r)=\left\lceil\frac{d_{\text {slice }}}{v_{\text {blood }}(r) \cdot T R}\right]
\end{aligned}
$$

Finally, a weighted summation of the MR signal is calculated according to

$$
M_{x y}\left(r, t_{b l o o d}, t, c_{C M}\right)=\sum_{n}^{n_{\max }} w_{n}(r) \cdot M_{x y}\left(t_{b l o o d}, n, t, c_{C M}\right)
$$

with weighting factors $w_{n}$ representing the fraction of spins at radial position $r$ within the imaging slice that experience $n$ excitations,

$$
w_{n}(r)= \begin{cases}\frac{T R \cdot v_{\text {blood }}(r)}{d_{\text {slice }}} & n<n_{\text {max }} \\ \frac{d_{\text {slice }}-(n-l) T R \cdot v_{\text {blood }}(r)}{d_{\text {slice }}} & n=n_{\text {max }}\end{cases}
$$

After contrast administration, the longitudinal $T_{1}$ and transverse $T_{2}$ relaxation times are shortened and the relative signal enhancement $(\Xi)$ of the voxel is given by

$$
\Xi\left(r, c_{C M}\right)=\frac{M_{x y}\left(r, t_{\text {blood }}, T E, c_{C M}\right)-M_{x y}\left(r, t_{\text {blood }}, T E, 0\right)}{M_{x y}\left(r, t_{\text {blood }}, T E, 0\right)}
$$

The relative signal enhancement as described in the equations above is solved numerically using the CM concentration as obtained by the generalized group averaged ph-VIF curve determined in the jugular vein and assuming a blood flow with a Poiseuille profile and a maximum 
blood velocity of $10 \mathrm{~cm} / \mathrm{s}$ within the vessel, which is comparable to the end-diastolic velocity in the common carotid artery(38) and the blood velocity in the jugular vein.

\section{Phantom measurements}

The effect of blood velocity on the magnitude and phase MR signals and the subsequent determination of CM concentration were investigated using a custom build flow phantom that consisted of tubing with an internal diameter of $20 \mathrm{~mm}$ connected to a pump with adjustable current. The tubing was positioned parallel to the main magnetic field in the MRI (3T whole body MRI system, Achieva, Philips Healthcare, Best, The Netherlands) with a dedicated 32-channel cardiac coil and imaging was performed using a TI-FFE sequence with the following imaging parameters: TR/TE $22.03 / 8.32 \mathrm{~ms}$, FA $35^{\circ}$, FOV $230 \times 230 \mathrm{~mm}$, acquisition matrix $382 \times 384$, reconstructed to a matrix size of $576 \times 576$ resulting in an acquired and reconstructed pixel size of $0.60 \times 0.60$ and $0.40 \times 0.40 \mathrm{~mm}$, respectively. A single transverse slice with a thickness of $1.5 \mathrm{~mm}$ was acquired. In addition, a spatial saturation slab with thickness of $60 \mathrm{~mm}$ was positioned upstream compared to the imaging slice parallel at a distance of 15 $\mathrm{mm}$ parallel to the imaging plane. Imaging was performed both without flow and under two static flow conditions $\left(v_{\text {mean }}=7.9\right.$ and $\left.12.9 \mathrm{~cm} / \mathrm{s}\right)$. Manganese chloride $(0.05 \mathrm{mM})$ was used as a solvent in order to lower the $T$, relaxation time of water to approximate the $T$, relaxation time of blood. Then, Gadobutrol (Gadovist, Bayer HealthCare, Berlin, Germany) was added in five linear steps from 0 to $4.4 \mathrm{mM}$ to the phantom and, after complete mixture of the gadobutrol, imaging was repeated. To examine the effect of blood velocity, data were analyzed for five radial regions, based on their distance to the center, separately. For analysis of the phase MR signal, a ring-shaped region surrounding the vessel was used as reference region in order to correct for background phase differences between different acquisitions.

\section{$\underline{\text { In vivo measurements }}$}

To illustrate the inflow effects, the MRI signal was analyzed according to the radial position for both the m-VIF and ph-VIF. For this, the luminal center of gravity was determined from the manually drawn contours and voxels were divided into five radial positions according to their relative distance to the center of gravity. For each radial position, the relative signal enhancement and phase difference were determined from the magnitude and phase MR images, respectively. Finally, these parameters were converted to the CM concentrations according to equations (2) and (5), respectively. 


\section{RESULTS}

\section{Determination of the vascular input function}

In Figure I, typical magnitude and phase MR images of the high temporal resolution scan are depicted before and after contrast injection. The signal in the lumen of the jugular vein is suppressed in the precontrast magnitude images due to the use of the saturation slab. After contrast injection, the signal in the lumen of the jugular vein is enhanced. The phase images show an enhancement of the signal after contrast injection, in both the jugular vein and the carotid artery. A generalized group averaged input function was determined using the average luminal signal for the jugular vein (average diameter $10.2 \pm 2.8 \mathrm{~mm}$ ) using both the magnitude and phase-based method (shown in Figure 2) and for the carotid artery (average diameter 8.2 \pm I.3 $\mathrm{mm}$ ) using the phase-based method. In the current study, the magnitude based VIF could not be determined reliably for the carotid artery since the signal intensity of the carotid lumen is already bright in the precontrast image [Figure I(A)]. ph-VIFs of the carotid artery and jugular vein show strong similarities. As expected, contrast arrival in the jugular vein appears delayed and dispersed in comparison to the carotid artery. For the jugular vein, peak CM concentrations derived from the ph-VIF were found to be on average fourfold increased compared to the $\mathrm{m}-\mathrm{VIF}(\mathrm{p}<0.00 \mathrm{I})$. The relative intersubject variations were $(\mathrm{I}) \mathrm{I} \mathrm{I} .0 \%$ (ph-VIF carotid artery), (2) $14.7 \%$ (ph-VIF jugular vein), and (3) $19.7 \%$ (m-VIF jugular vein).

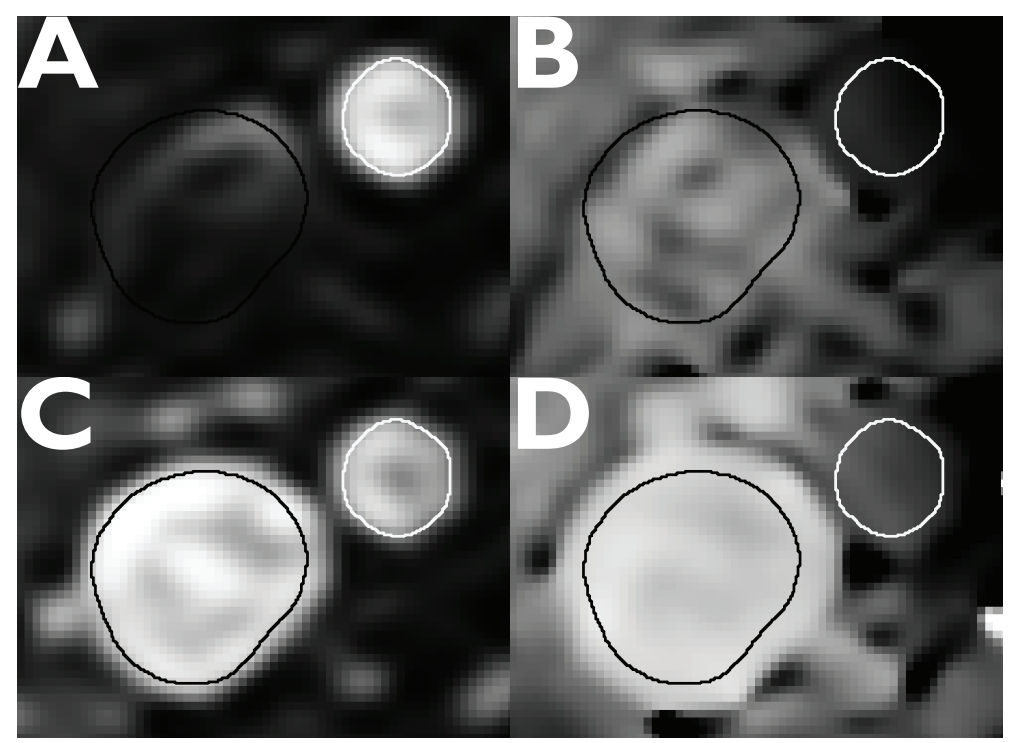

\section{Figure I}

Example images from the internal jugular vein (black contour line) and common carotid artery (white contour line) for the signal enhancement in both the magnitude $(A)$ and $(C)$ and phase (B) and (D) MR images from a patient with a carotid stenosis of $50 \%$ in the internal carotid artery (as measured with Doppler ultrasound). Before contrast injection (A) and (B), the lumen of the carotid artery (white contour) appears bright in the magnitude MR image (A) while the lumen of the jugular vein (black contour) is dark due to the presence of the spatial saturation slab (cranially). After contrast injection (C) and (D), the magnitude MR signal (C) in the jugular vein is enhanced due to shortening of the blood relaxation time in the presence of the Gd contrast medium (CM). For the phase MR images (B) and (D), an increase in phase is seen after (D) compared to before (B) contrast injection, in both the carotid artery and jugular vein, due to the change of the magnetic susceptibility in the presence of the CM. 


\section{DCE-MRI of carotid plaque: influence on model parameters}

Significant correlations were found between quantitative model parameters $\left(K^{\text {trans }}\right.$ and $\left.v_{p}\right)$ using the three VIFs ( $m$-VIF jugular vein, ph-VIF jugular vein, and ph-VIF carotid artery, Figure 3 and Table I), which ranged from 0.93 to 0.99 for $K^{\text {trans }}$ and from 0.58 to 0.90 for $v_{p}$. $A$ considerable difference in absolute values was found between the quantitative parameters, indicating dependency on the used VIF.

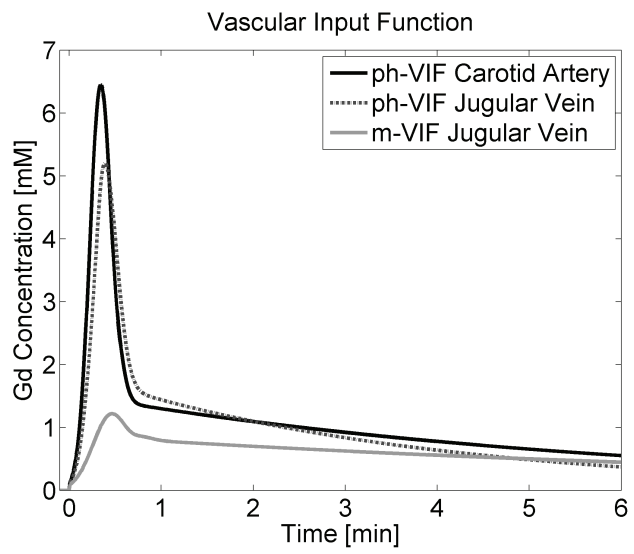

\section{Figure 2}

Population averaged vascular input functions from the carotid artery (phase) and the jugular vein (phase and magnitude).
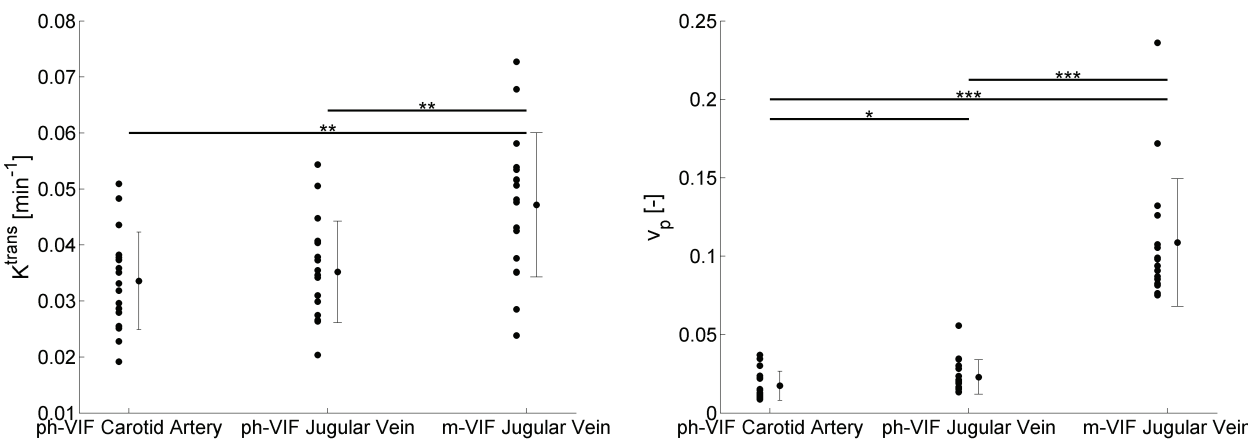

\section{Figure 3}

Parameter agreement for the quantitative parameters $K^{\text {trans }}$ (left figure) and $v_{p}$ (right figure) determined using the three different VIFs (shown in Figure 2). " $\mathrm{p}$-value $<0.05$, " $\mathrm{p}$-value $<0.0 \mathrm{I}$, " $\mathrm{p}$-value $<0.00 \mathrm{I}$. Mean and standard deviation are indicated with error bars next to the individual points. 


\section{Estimation of effect of local blood inflow on signal enhancement}

Simulations

Analytical analysis of equation (14) showed that, with the imaging parameters described for the high temporal resolution DCE-MRI acquisition, blood with a velocity lower than $6 \mathrm{~cm} / \mathrm{s}$ will experience multiple excitations. From the calculated relative signal enhancement curves (Figure 4), it is clear that near the vessel wall, where the blood velocity is low, the magnitude MR signal is saturated at CM concentrations of approximately $2 \mathrm{mM}$; the first pass peak of the CM looks blunted in the resulting curve. This phenomenon is less pronounced (though still visible) with an increased blood flow velocity, i.e., at the center of the lumen.

\section{Table I}

Parameter agreement for $\mathrm{K}^{\text {trans }}$ and $\mathrm{v}_{\mathrm{p}}$ between the three different vascular input functions determined in the current study (a phase-based vascular input function in the carotid artery (ph-VIF CA), and the jugular vein (ph-VIF JV), and a magnitude-based vascular input function in the jugular vein ( $m$-VIF JV)). P-values were calculated using a Wilcoxon signed rank test, and Spearman's correlation coefficient was calculated.

\begin{tabular}{lllll}
\hline & \multicolumn{3}{l}{ P-value (Wilcoxon singed rank test) } & $R$ (Spearman) \\
& $K^{\text {trans }}\left[\mathrm{min}^{-1}\right]$ & $\mathrm{v}_{\mathrm{p}}$ & $\mathrm{K}^{\text {trans }}\left[\mathrm{min}^{-1}\right]$ & $\mathrm{v}_{\mathrm{p}}$ \\
\hline ph-VIF CA vs ph-VIF JV & $<0.00 \mathrm{I}$ & $<0.0 \mathrm{I}$ & $0.99(\mathrm{p}<0.00 \mathrm{I})$ & $0.90(\mathrm{p}<0.00 \mathrm{I})$ \\
ph-VIF CA vs m-VIF JV & $<0.00 \mathrm{I}$ & $<0.00 \mathrm{I}$ & $0.93(\mathrm{p}<0.00 \mathrm{I})$ & $0.58(\mathrm{p}<0.05)$ \\
ph-VIF JV vs m-VIF JV & $<0.00 \mathrm{I}$ & $<0.00 \mathrm{I}$ & $0.94(\mathrm{p}<0.00 \mathrm{I})$ & $0.72(\mathrm{p}<0.0 \mathrm{I})$ \\
\hline
\end{tabular}

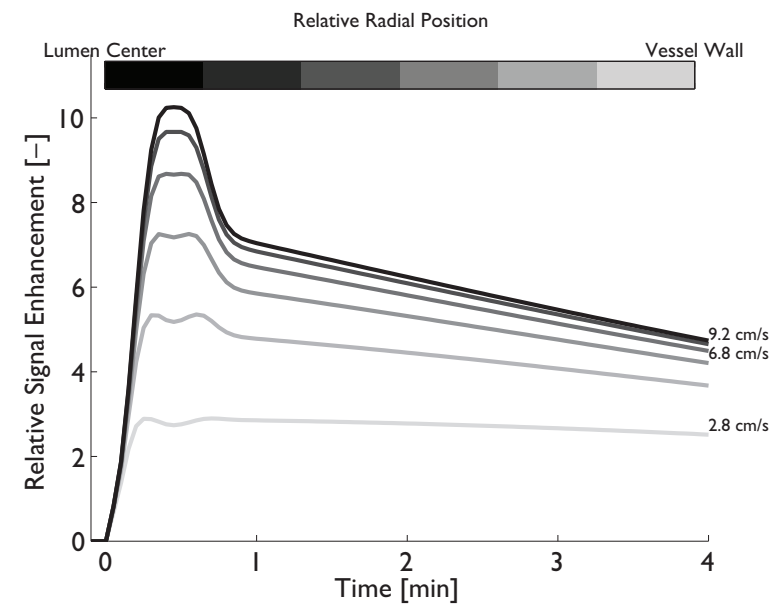

\section{Figure 4}

Relative signal enhancement curves for a theoretical blood vessel with a Poisseuille blood flow profile (maximum velocity of $10 \mathrm{~cm} / \mathrm{s}$ ) calculated based on the Bloch equations and with gadolinium concentrations derived from the ph-VIF of the jugular vein (Figure 2) as concentration input. Signal saturation of the first pass peak can clearly be observed, especially for lower blood velocities. For lower blood velocities, signal saturation occurs even at low concentrations, i.e., after the first pass peak, while this is less pronounced for voxels with a higher velocity, closer to the center of the lumen. 


\section{Phantom}

The MR magnitude and phase signal have been plotted as a function of CM concentration for various radial regions, corresponding with different velocities in Figure 5. The results showed a nonlinear relationship between the CM concentration and the relative magnitude MR signal, while for the phase difference, a linear relationship was observed. In the presence of flow, this underestimation of the magnitude MR signal will result in an underestimation of the CM concentration, especially for decreasing velocity, while this will not occur when using the phase MR signal.

\section{$\underline{\text { In vivo }}$}

In Figure 6(A), the CM concentration determined from magnitude MR images as function of the radial position is depicted. Pixels located far from the center of mass, i.e., at a radial distance of $7 \mathrm{~mm}$, had on average a lower signal enhancement and thus lower calculated CM concentration, compared to the pixels in the center. Moreover, the first pass peak is hardly discernible. Figure 6(B) shows the CM concentration determined using the phase MR images. Note that in this case, the CM concentration was similar at every radial position and the first pass peak is clearly visible. Both were measured in the jugular vein of the same subject.
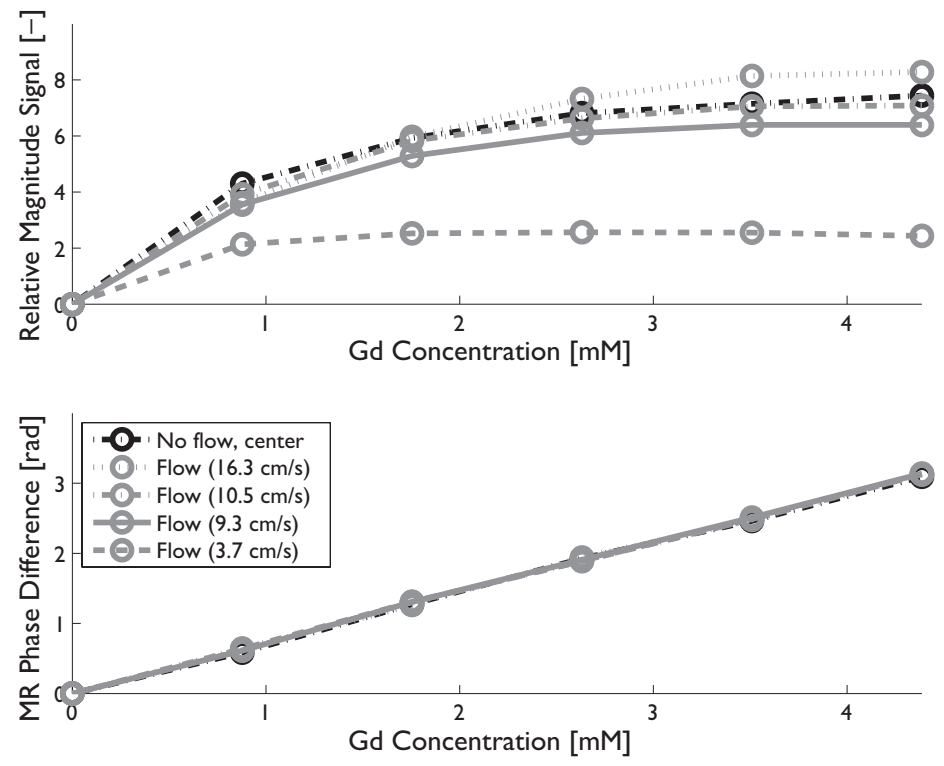

\section{Figure 5}

Experimentally determined relative signal enhancement and phase difference using either the magnitude or phasebased method in the condition of no flow and with flow. Analyses with flow were performed for several radial positions within the tube, corresponding to different flow velocities ( $v=16.3,10.5,9.3$, and $3.7 \mathrm{~cm} / \mathrm{s}$ ). For the magnitude MR signal, a flow velocity dependent effect can be seen in the curves, while for the phase MR signal, this effect is clearly not visible. 

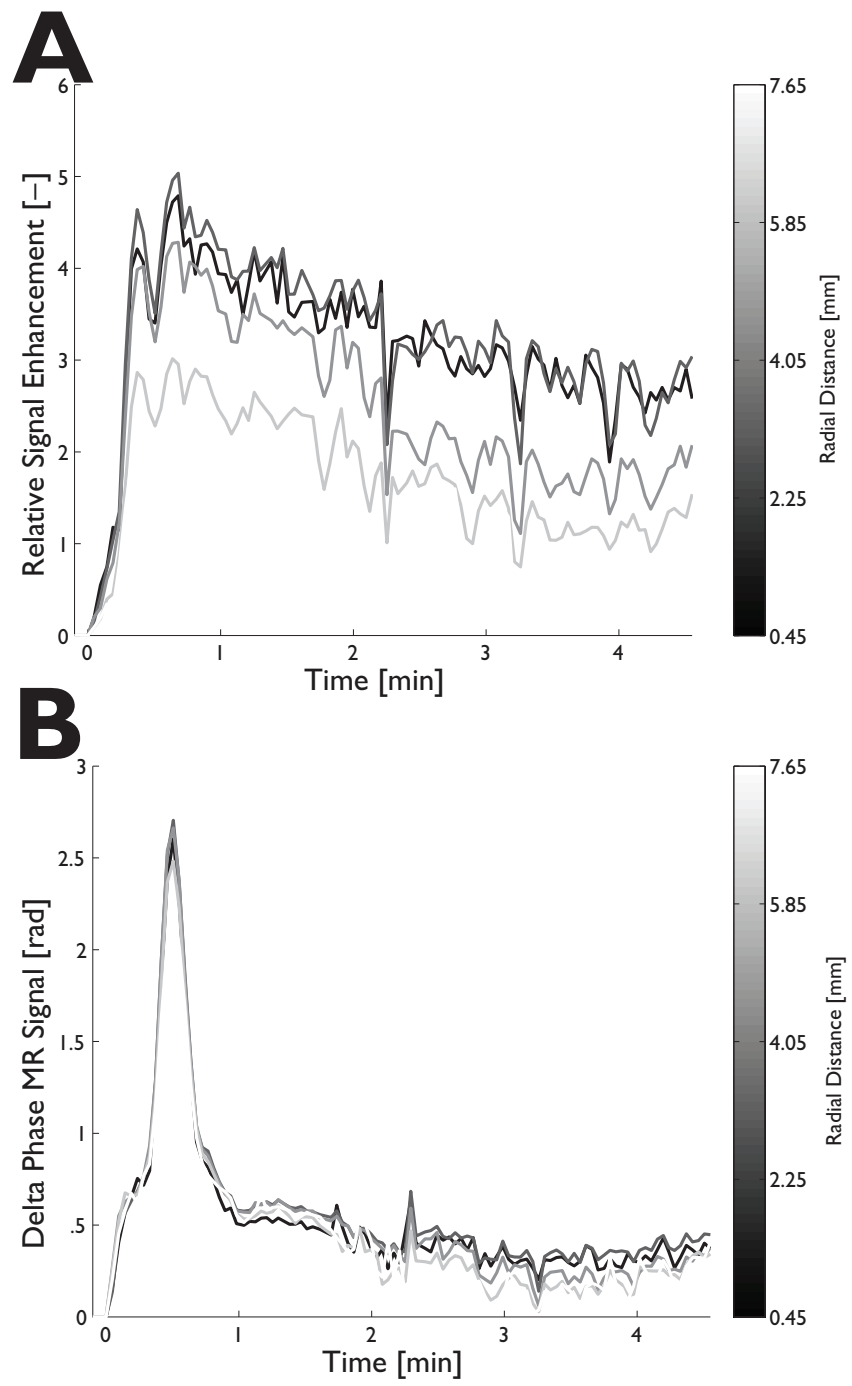

\section{Figure 6}

Experimentally measured concentration curves determined using (A) magnitude $M R$ images, and (B) phase signal change using phase MR images in the jugular vein of a single patient. Pixels were grouped according to their radial distance (reconstructed resolution) to the center of the lumen. In agreement with the simulations and flow phantom experiments, an underestimation of the CM concentration is found using the magnitude MR images with increasing radial distance to the center of the lumen. This dependency on radial position (and thus local blood velocity) is not seen when the phase MR images were used. 


\section{Discussion}

In the present study, we have demonstrated a significant underestimation of the peak CM concentrations in magnitude based m-VIF, compared to the phase-based ph-VIF. The underestimation of the first pass peak concentration of $\mathrm{m}$-VIF with the current imaging and contrast injection protocol was approximately fourfold. Simulations, phantom, and in vivo experiments revealed that the amount of underestimation of the CM concentration based on the magnitude MR signal is related to the blood velocity and thus to the location relative to the center of the vessel. This resulted in signal saturation at higher CM concentrations and lower local blood velocities. In contrast, as demonstrated by the phantom experiments, the phase-based VIF was not affected by flow. This resulted in a phase difference independent of the radial position relative to the center of the vessel in both the phantom and in vivo experiments. The phase-based method allowed determination of the VIF from both the carotid artery as well as the jugular vein. Previously, it was shown that the phase-based method is less influenced by the measurement location within a $3 \mathrm{D}$ volume compared to the magnitude-based method (26), but, as far as we are aware of, the dependency of m-VIF on the radial position due to different local blood velocities within the vessel was not previously shown (29). A low blood velocity results in an increased longitudinal relaxation of the MR signal between the saturation and imaging slice, leading to less relative signal enhancement after contrast injection in the magnitude images and thus an underestimation of the CM concentration of the $\mathrm{m}-\mathrm{VIF}$. Previous research (39) has focused on the effect of (unsteady) blood flow in the vessel on the CM concentration. Despite the substantial differences in calculated CM concentration, significant correlations were found between the pharmacokinetic parameters, determined by either a m-VIF or ph-VIF, with Spearman correlation coefficients ranging from 0.93 to 0.99 (all $P$-value $<0.00 \mathrm{I}$ ) for $\mathrm{K}^{\text {trans }}$ and 0.58 to 0.90 for $\mathrm{v}_{\mathrm{p}}$ (all $\mathrm{P}$-values $<0.05$ ). Previous studies $(\mathrm{II}, \mathrm{I} \mathrm{I})$, in which DCE-MRI was related to histologically determined plaque features, used an $\mathrm{m}-\mathrm{VIF}$ for the pharmacokinetic analysis. It is likely that an underestimation of the VIF has occurred, due to the blood flow effects as described in the current study. Nevertheless, due to the strong correlation between the model parameters when using different VIFs, similar results regarding the association between DCE-MRI and histology can be expected with the use of different VIFs. However, considerable differences were found between absolute values of quantitative parameters when using different VIFs. From this, we conclude that care should be taken when comparing absolute values across different studies. Thus, in order to determine appropriate thresholds of model parameters for patient risk stratification, the use of a ph-VIF is recommended. Taking into account the challenges that accompany reliable determination of a patient-specific VIF, we chose to generate a population-averaged VIF for the analysis of the microvasculature in the atherosclerotic carotid plaques using a separate DCE-MRI scan with high temporal resolution. Previous studies that compared population-averaged VIFs to patient specific AIFs showed an increased (37) or comparable (29) reproducibility of DCE-MRI derived parameters for population averaged AIFs. A patient-specific VIF (40) could account for inter individual variations (25). Atherosclerotic plaques in the carotid artery have a relatively small size and, hence, a high spatial resolution is crucial. As a result, the temporal resolution is limited and therefore accurate determination of the first pass peak of the VIF immediately after CM injection is impossible with the high spatial resolution MRI pulse sequence. The injection rate of the CM is low compared to previously performed studies which investigate 
atherosclerotic plaques. However, since CM uptake in the plaque is rather slow, i.e., low $\mathrm{K}^{\text {trans }}$ values $\left(<0.2 \mathrm{~min}^{-1}\right)$ are expected (I5), a slower injection rate may be applied (4I). The slow CM uptake also justifies the use of a high spatial resolution at the cost of temporal resolution. The phase signal is likely to contain some sources of error itself. Motion-induced phase shifts and accumulation of contrast agent in background tissue regions have been identified as the main sources of uncertainty in phase-based VIFs (24). A method to correct for this problem has been proposed previously (24) in which a circular region around the region-of-interest was used for the correction of CM accumulation. This method is applicable in brain perfusion studies; however, in our study this approach was not feasible due to the anatomy of the neck, in which many blood vessels and glands are present. Therefore, we used a different, also commonly used, method ( $3 \mathrm{I}$ ) by using the phase signal from a muscle. Previous research using this method demonstrated that the phase-based vascular input function corresponded very good with a vascular input function measured using DCE-CT, while for the magnitude-based method an anomalous shape and incorrect height were observed. In addition, contrast uptake in the muscle is low compared to the blood plasma concentration especially during first passage of the bolus of the contrast medium (42). For the magnitude-based method, the Ernst equation is used for the conversion from relative signal enhancement to CM concentration, as was done in previous research comparing magnitude- and phase-based VIFs (29,3I). However, due to the blood flow within the vessel, the number of signal excitations is not always sufficient to reach the assumed steady state. The resulting deviation will lead to an additional uncertainty in determination of the CM concentration. For the simulations performed in the current study, we did take into account the number of excitations for the different blood velocities. However, in vivo, this would require additional blood velocity measurements using, for example, a quantitative phase contrast MRI acquisition, which would be impractical in a daily clinical setting. An additional advantage of the phase-based method is the fact that no saturation slab is required in the pulse sequence, while this saturation slab is necessary for the magnitude-based method. This was also the reason that $\mathrm{m}$-VIF could not be determined in the carotid artery for the present study. An additional saturation slab at the caudal position, which would enable measurement of the m-VIF in the common carotid artery, would lead to a decreased temporal resolution, which is unfavorable for accurate determination of the first pass peak from the CM. Eliminating the saturation slab from the MRI pulse sequence can lead to an improvement of the temporal resolution. In the current study, the saturation pulse might also have lowered the signal-to-noise ratio for the phase-based measurement in the jugular vein. Since the goal of the present study was to compare both techniques, the MR protocol was chosen such that it allowed analysis of both m-VIF and ph-VIF. For example, an increased echo time would increase the accuracy for the ph-VIF determination; however, this would lead to increased $\mathrm{T}_{2}{ }^{*}$ effects on the magnitude MR images. Placement of the saturation slab more closely to the imaging slice will decrease the dependency of the signal enhancement on local blood velocity but might lead to a decrease in signal-to-noise ratio of the phase and magnitude MR images. 


\section{CONCLUSIONS}

Concluding, our study illustrates the importance of using a phase-based VIF for quantitative modeling of DCE-MRI, since the magnitude-based VIF is strongly affected by the local blood flow-velocity in the vessel, resulting in a large underestimation of the CM concentration. We have demonstrated that signal saturation of the magnitude MRI signal from within a blood vessel is also found within a single imaging slice. Simulations and phantom experiments linked this to the local blood velocity within the vessel. Phantom experiments showed no influence of flow on the determination of the CM concentration when making use of the phase MR signal. The use of a ph-VIF therefore results in more realistic value for the quantitative parameters $\left(K^{\text {trans }}\right.$ and $\left.v_{p}\right)$. This will further increase the potential value of quantitative DCE-MRI for use in a daily clinical practice, both for carotid plaque imaging and in other areas where DCE-MRI is commonly applied. 


\section{REFERENCES}

I. Muller JE, Abela GS, Nesto RW, Tofler GH. Triggers, Acute Risk Factors and Vulnerable Plaques: The Lexicon of a New Frontier. J Am Coll Cardiol 1994;23:809-8I3.

2. Virmani R, Kolodgie FD, Burke AP, Farb A, Schwartz SM. Lessons from Sudden Coronary Death: A Comprehensive Morphological Classification Scheme for Atherosclerotic Lesions. Arterioscler Thromb Vasc Biol 2000;20: 1262-1275.

3. Davies MJ, Richardson PD, Woolf N, Katz DR, Mann J. Risk of Thrombosis in Human Atherosclerotic Plaques: Role of Extracellular Lipid, Macrophage, and Smooth Muscle Cell Content. British heart journal 1993;69:377381.

4. Saba L, Anzidei M, Marincola BC, Piga M, Raz E, Bassareo PP et al. Imaging of the Carotid Artery Vulnerable Plaque Cardiovascular and interventional radiology 20।4;37:572-585.

5. Gupta A, Baradaran H, Schweitzer AD, Kamel H, Pandya A, Delgado D et al. Carotid Plaque Mri and Stroke Risk: A Systematic Review and Meta-Analysis. Stroke 20I3;44:307I-3077.

6. Sluimer JC, Gasc JM, van Wanroij JL, Kisters N, Groeneweg M, Sollewijn Gelpke MD et al. Hypoxia, HypoxiaInducible Transcription Factor, and Macrophages in Human Atherosclerotic Plaques Are Correlated with Intraplaque Angiogenesis. J Am Coll Cardiol 2008;5 I:I 258-I 265.

7. Virmani R, Kolodgie FD, Burke AP, Finn AV, Gold HK, Tulenko TN et al. Atherosclerotic Plaque Progression and Vulnerability to Rupture: Angiogenesis as a Source of Intraplaque Hemorrhage. Arterioscler Thromb Vasc Biol 2005;25:2054-206I.

8. Kolodgie FD, Gold HK, Burke AP, Fowler DR, Kruth HS, Weber DK et al. Intraplaque Hemorrhage and Progression of Coronary Atheroma. N Engl J Med 2003;349:2316-2325.

9. Chen H, Cai J, Zhao X, Underhill H, Ota H, Oikawa M et al. Localized Measurement of Atherosclerotic Plaque Inflammatory Burden with Dynamic Contrast-Enhanced Mri. Magn Reson Med 2010;64:567-573.

10. Dong L, Kerwin WS, Chen H, Chu B, Underhill HR, Neradilek MB et al. Carotid Artery Atherosclerosis: Effect of Intensive Lipid Therapy on the Vasa Vasorum-Evaluation by Using Dynamic Contrast-Enhanced Mr Imaging. Radiology 201 I;260:224-23I.

II. Kerwin W. Quantitative Magnetic Resonance Imaging Analysis of Neovasculature Volume in Carotid Atherosclerotic Plaque. Circulation 2003;107:85I-856.

12. Kerwin WS, O'Brien KD, Ferguson MS, Polissar N, Hatsukami TS, Yuan C. Inflammation in Carotid Atherosclerotic Plaque: A Dynamic Contrast-Enhanced Mr Imaging StudyI. Radiology 2006;24I:459-468.

13. Kerwin WS, Oikawa M, Yuan C, Jarvik GP, Hatsukami TS. Mr Imaging of Adventitial Vasa Vasorum in Carotid Atherosclerosis. Magn Reson Med 2008;59:507-5I4.

14. Sun J, Song Y, Chen H, Kerwin WS, Hippe DS, Dong L et al. Adventitial Perfusion and Intraplaque Hemorrhage: A Dynamic Contrast-Enhanced Mri Study in the Carotid Artery. Stroke 2013;44: I03 I- 1036.

15. Gaens ME, Backes WH, Rozel S, Lipperts M, Sanders SN, Jaspers K et al. Dynamic Contrast-Enhanced Mr Imaging of Carotid Atherosclerotic Plaque: Model Selection, Reproducibility, and Validation. Radiology 2013;266:27I-279.

16. Khalifa F, Soliman A, El-Baz A, Abou El-Ghar M, El-Diasty T, Gimel'farb G et al. Models and Methods for Analyzing Dce-Mri: A Review. Med Phys 2014;41:12430I.

17. Bergamino M, Bonzano L, Levrero F, Mancardi GL, Roccatagliata L. A Review of Technical Aspects of TIWeighted Dynamic Contrast-Enhanced Magnetic Resonance Imaging (Dce-Mri) in Human Brain Tumors. Physica medica : PM : an international journal devoted to the applications of physics to medicine and biology : official journal of the Italian Association of Biomedical Physics 20 14;30:635-643.

18. Ingrisch M, Sourbron S. Tracer-Kinetic Modeling of Dynamic Contrast-Enhanced Mri and Ct: A Primer. J Pharmacokinet Pharmacodyn 20 I3;40:28I-300. 
19. Garpebring A, Wirestam R, Ostlund N, Karlsson M. Effects of Inflow and Radiofrequency Spoiling on the Arterial Input Function in Dynamic Contrast-Enhanced Mri: A Combined Phantom and Simulation Study. Magn Reson Med 2011;65:1670-1679.

20. Kotys MS, Akbudak E, Markham J, Conturo TE. Precision, Signal-to-Noise Ratio, and Dose Optimization of Magnitude and Phase Arterial Input Functions in Dynamic Susceptibility Contrast Mri. J Magn Reson Imaging 2007;25:598-6II.

21. Akbudak E, Conturo TE. Arterial Input Functions from Mr Phase Imaging. Magn Reson Med 1996;36:809-8I5.

22. Akbudak E, Norberg RE, Conturo TE. Contrast-Agent Phase Effects: An Experimental System for Analysis of Susceptibility, Concentration, and Bolus Input Function Kinetics. Magn Reson Med 1997;38:990- 1002.

23. Bleeker EJ, van Buchem MA, Webb AG, van Osch MJ. Phase-Based Arterial Input Function Measurements for Dynamic Susceptibility Contrast Mri. Magn Reson Med 2010;64:358-368.

24. Garpebring A, Wirestam R, Yu J, Asklund T, Karlsson M. Phase-Based Arterial Input Functions in Humans Applied to Dynamic Contrast-Enhanced Mri: Potential Usefulness and Limitations. Magma 20I I;24:233-245.

25. van Osch MJ, Vonken EJ, Viergever MA, van der Grond J, Bakker CJ. Measuring the Arterial Input Function with Gradient Echo Sequences. Magn Reson Med 2003;49: I067- 1076.

26. van Osch MJ, van der Grond J, Bakker CJ. Partial Volume Effects on Arterial Input Functions: Shape and Amplitude Distortions and Their Correction. J Magn Reson Imaging 2005;22:704-709.

27. van Osch MJ, Vonken EJ, Bakker CJ, Viergever MA. Correcting Partial Volume Artifacts of the Arterial Input Function in Quantitative Cerebral Perfusion Mri. Magn Reson Med 200I;45:477-485.

28. Conturo TE, Akbudak E, Kotys MS, Chen ML, Chun SJ, Hsu RM et al. Arterial Input Functions for Dynamic Susceptibility Contrast Mri: Requirements and Signal Options. J Magn Reson Imaging 2005;22:697-703.

29. Cron GO, Foottit C, Yankeelov TE, Avruch LI, Schweitzer ME, Cameron I. Arterial Input Functions Determined from Mr Signal Magnitude and Phase for Quantitative Dynamic Contrast-Enhanced Mri in the Human Pelvis. Magn Reson Med 201 I;66:498-504.

30. Cron GO, Wallace JC, Stevens WD, Fortin T, Pappas BA, Wilkins RC et al. A Comparison of T2*-Weighted Magnitude and Phase Imaging for Measuring the Arterial Input Function in the Rat Aorta Following Intravenous Injection of Gadolinium Contrast Agent. Magn Reson Imaging 2005;23:619-627.

31. Korporaal JG, van den Berg CA, van Osch MJ, Groenendaal G, van Vulpen M, van der Heide UA. PhaseBased Arterial Input Function Measurements in the Femoral Arteries for Quantification of Dynamic ContrastEnhanced (Dce) Mri and Comparison with Dce-Ct. Magn Reson Med 201 I;66: I 267-I 274.

32. Truijman MT, Kooi ME, van Dijk AC, de Rotte AA, van der Kolk AG, Liem MI et al. Plaque at Risk (Parisk): Prospective Multicenter Study to Improve Diagnosis of High-Risk Carotid Plaques. International journal of stroke : official journal of the International Stroke Society 20।4;9:747-754.

33. North American Symptomatic Carotid Endarterectomy Trial. Methods, Patient Characteristics, and Progress. Stroke 1991;22:711-720.

34. Randomised Trial of Endarterectomy for Recently Symptomatic Carotid Stenosis: Final Results of the Mrc European Carotid Surgery Trial (Ecst). Lancet 1998;35 I: I379- I387.

35. Schabel MC, Parker DL. Uncertainty and Bias in Contrast Concentration Measurements Using Spoiled Gradient Echo Pulse Sequences. Phys Med Biol 2008;53:2345-2373.

36. Stanisz GJ, Odrobina EE, Pun J, Escaravage M, Graham SJ, Bronskill MJ et al. TI, T2 Relaxation and Magnetization Transfer in Tissue at 3t. Magn Reson Med 2005;54:507-5 I2.

37. Parker GJ, Roberts C, Macdonald A, Buonaccorsi GA, Cheung S, Buckley DL et al. Experimentally-Derived Functional Form for a Population-Averaged High-Temporal-Resolution Arterial Input Function for Dynamic Contrast-Enhanced Mri. Magn Reson Med 2006;56:993-1000.

38. Brands PJ, Hoeks AP, Hofstra L, Reneman RS. A Noninvasive Method to Estimate Wall Shear Rate Using Ultrasound. Ultrasound Med Biol 1995;21:I71-185. 
39. Peeters F, Annet L, Hermoye L, Van Beers BE. Inflow Correction of Hepatic Perfusion Measurements Using TIWeighted, Fast Gradient-Echo, Contrast-Enhanced Mri. Magn Reson Med 2004;5 I:7 I0-7I 7.

40. Ashton E, Raunig D, Ng C, Kelcz F, McShane T, Evelhoch J. Scan-Rescan Variability in Perfusion Assessment of Tumors in Mri Using Both Model and Data-Derived Arterial Input Functions. J Magn Reson Imaging 2008;28:79 I 796.

4I. Aerts HJ, van Riel NA, Backes WH. System Identification Theory in Pharmacokinetic Modeling of Dynamic Contrast Enhanced Mri: Influence of Contrast Injection. Magn Reson Med 2008;59: I I I - I I I 9.

42. Jaspers K, Leiner T, Dijkstra P, Oostendorp M, van Golde JM, Post MJ et al. Optimized Pharmacokinetic Modeling for the Detection of Perfusion Differences in Skeletal Muscle with Dce-Mri: Effect of Contrast Agent Size. Med Phys 2010;37:5746-5755. 


\section{Abstract}

\section{Objectives}

The dynamic contrast-enhanced (DCE-)MRI parameter $\mathrm{K}^{\text {trans }}$ is frequently used to study atherosclerotic plaque microvasculature. $K^{\text {trans }}$ has been reported using different descriptive statistics (mean, median, $75^{\text {th }}$ percentile) either for the whole vessel wall or the adventitia in previous studies. Present objectives were to assess parameter agreement of $\mathrm{K}^{\text {trans }}$ between the two vascular regions and to study the correlation with microvessel density on histology.

\section{Material and methods}

DCE-MRI parameter agreement was analyzed in I I 0 symptomatic patients with $\geq 2 \mathrm{~mm}$ carotid plaque that underwent a 3T carotid (DCE-)MRI examination. $\mathrm{K}^{\text {trans }}$ was estimated in the entire vessel wall and adventitia. Twenty-three patients underwent carotid endarterectomy and were used for comparison with histological quantification of microvessel density of the plaque using CD3I immunohistochemistry. DCE-MRI parameters in the vessel wall regions were compared using Pearson's correlation coefficient, Bland-Altman analysis, and a two-sided paired samples T-test. Correlation of the DCE-MRI parameters with histology was studied using the Pearson's correlation coefficient.

\section{Results}

Median adventitial $K^{\text {trans }}$ was $5 \%$ higher $(p=0.003)$ than entire vessel wall $K^{\text {trans }}$, with no differences for other descriptive statistics. Vessel wall and adventitial $\mathrm{K}^{\text {trans }}$ showed similar moderately strong correlations with plaque microvessel density on histology (Pearson's $\rho: 0.59-0.65$ $(p<0.003)$ and 0.52-0.64 $(p<0.01 \mathrm{I})$, respectively).

\section{Conclusions}

The similar moderately strong correlations for vessel wall and adventitial $\mathrm{K}^{\text {trans }}$ with microvessel density on histology suggested that both regions reflected plaque microvessel density. Care should to be taken when comparing absolute values between studies. Future studies incorporating thresholds for risk stratification need to agree upon standardization of DCE-MRI parameters. 


\section{INTRODUCTION}

Rupture of a vulnerable carotid atherosclerotic plaque may lead to an ischemic stroke, resulting in morbidity or even death of the patient (I). Several plaque features have been identified as important hallmarks of plaque vulnerability, including increased plaque microvasculature (2). These microvessels originate from the adventitia (the exterior part of the vessel wall) and grow into the plaque tissue (3), supplying the plaque with nutrients and oxygen. The structural integrity of plaque microvasculature endothelium is impaired (4). Therefore, plaque microvessels may provide an entry point for inflammatory cells and erythrocytes and may cause destabilization of the plaque (5), leading to an increased risk of plaque rupture.

Dynamic contrast-enhanced (DCE)-MRI has emerged as a non-invasive technique to assess plaque microvasculature. With this technique, the microvasculature can be studied (semi-) quantitatively by studying the leakage of the contrast medium (CM) from the microvasculature into the plaque tissue. Previous research has been performed in specimens from patients undergoing a carotid endarterectomy (CEA). During CEA, the carotid plaque (i.e. the intima and (part of) the media is removed, leaving the remaining vessel wall including the adventitia in situ. These studies have already shown a correlation between the volume transfer coefficient, $\mathrm{K}^{\text {trans }}$, a quantitative DCE-MRI parameter reflecting microvessel flow, density, and permeability, and histological measurements of the plaque microvasculature as a reference (6-9).

A number of studies have investigated the relationship between plaque microvasculature and other features of plaque vulnerability, such as inflammation (10-12) and the presence of intraplaque hemorrhage (13).The focus of these studies was either on the entire vessel wall $(6-8,10-12,14)$ or only on the outer layer of the vessel wall, the adventitia $(9,13)$. Currently, no direct comparison between quantitative DCE-MRI parameters from these two vascular regions has been performed. In addition, different descriptive statistics (e.g. mean, median, or $75^{\text {th }}$ percentile) have been reported in these various studies. While mean and median values may be more suited to describe the overall state of the plaque microvasculature, the $75^{\text {th }}$ percentile may be more suited to reflect specific hotspots of increased plaque microvascularity.

The aim of the present study was to systematically investigate the agreement between quantitative DCE-MRI pharmacokinetic parameters derived from the entire vessel wall and the adventitial region in the same patient and correlate these parameters to the intraplaque microvessel endothelium density in histological specimens as a reference. 


\section{Material ANd Methods}

\section{Study Population}

In this present prospective, cross-sectional study consecutive patients with an acute cerebrovascular event (stroke, transient ischemic attack (TIA), or amaurosis fugax) due to an ipsilateral carotid artery stenosis and a carotid plaque $\geq 2 \mathrm{~mm}$ on duplex ultrasonography were included. Patients with a severe comorbidity (dementia, severe heart failure, i.e. New York Heart Association class III-IV, severe pulmonary dysfunction dependent on oxygen supply, hemiparalysis, or complete aphasia) and standard contra-indications for MRI, such as ferromagnetic/ other electronic implants were excluded. Patients with severe renal disease (renal clearance $<30 \mathrm{ml} /$ minute) were not eligible for contrast-enhanced MRI and were therefore excluded from the current analysis. Medical history and medication use were ascertained at the time of subject enrolment. All study participants underwent carotid DCE-MRI of the symptomatic carotid plaque. Histological and MRI analysis was performed blinded to the other test results by randomization of the histological samples before analysis. Approval of the local Institutional Ethical Review Board was obtained. Written informed consent was obtained from all patients before study inclusion.

\section{MR imaging}

MR imaging was performed on a 3T whole body MRI system (Achieva, Philips Healthcare, Best, The Netherlands) using a dedicated 8-channel carotid RF coil (Shanghai Chenguan Medical Technologies Co., Shanghai, China). Five MRI pulse sequences were used to facilitate delineation of plaque boundaries as described previously (I5). For DCE-MRI, an end-diastolic ECG gated 3D TI-TFE MRI pulse sequence was acquired centered at the position of the highest plaque burden with the following parameters: repetition/echo time II.6/5.7 ms, flip angle $35^{\circ}$, Field of View $130 \times 130$ mm, acquisition/reconstruction matrix $208 \times 206 / 5$ I $2 \times 5$ I2, five adjoining transversal slices, slice thickness $2 \mathrm{~mm}$ (16). The temporal resolution was approximately 20 seconds per time frame (dependent on heart rate). At the beginning of the third time frame, a contrast medium (CM) $(0.1 \mathrm{mmol} / \mathrm{kg}$ Gadobutrol (Gadovist, Bayer HealtCare, Berlin, Germany)) was injected with a power injector (Spectris Solaris, Medrad, Warrendale, USA) at $0.5 \mathrm{ml} / \mathrm{sec}$ followed by a $20 \mathrm{ml}$ saline chaser at the same rate. DCE-MR imaging was continued for six minutes after contrast injection.

\section{CEA and histological preparation}

Indication for CEA was based on clinician's decision. Surgeons were instructed to remove the carotid plaque in one piece. Carotid endarterectomy specimens were collected after surgery and histological processing was performed as described previously (8). In short, after CEA, the carotid plaques were immediately fixed in $10 \%$ buffered formalin, transversely cut in $3 \mathrm{~mm}$ slices, decalcified, embedded in paraffin, and cut in $4 \mu \mathrm{m}$ (transverse) slices. Co-localization of histopathological sections with DCE-MR imaging slices was performed using the longitudinal position relative to the carotid bifurcation and/or the narrowest carotid artery lumen as a reference (8). Plaque microvasculature was detected on co-registered slices with immunohistochemistry using primary antibodies against CD3I (clone JC70A, Dako North America, Carpinteria, California), for identification of endothelial cells. 
Plaque microvasculature was quantified on high spatial resolution digital images by using morphometric analysis software (QWin V3, Leica, Cambridge, England). Plaque microvasculature was measured as the CD3 I-positive area surrounding a lumen. Relative density of microvessel endothelium was calculated by dividing the total CD3I-positive area by the total plaque area.

\section{DCE-MRI evaluation and pharmacokinetic modeling}

Both luminal and outer plaque contours were drawn as described previously (16). Luminal contours were drawn on the 3D TOF, pre-contrast TIw QIR TSE, post-contrast TIw QIR TSE, or T2W TSE MR images, in subsequent order. Outer vessel wall contours were drawn by using the pre-contrast TIw QIR TSE, post-contrast TIw QIR TSE, TIw TFE or T2w TSE $M R$ images, in subsequent order. Contours from anatomic MRI acquisitions were transferred to the DCE-MR images. If necessary, contours were manually adjusted and individual time frames shifted to correct for small patient displacements. Luminal contours were corrected to avoid partial volume effects, by keeping sufficient distance from the vessel lumen. Outer plaque contours were corrected to include the adventitial vasa vasorum, which shows increased enhancement after contrast material administration. The entire vessel wall region is defined as the region between the luminal and outer wall contours. Adventitial region of the vessel wall was determined according to previously introduced criteria (13). According to these criteria, all pixels within $0.625 \mathrm{~mm}$ of the outer wall contour in a region of the vessel wall with plaque, which was defined as a wall thickness greater than $1.5 \mathrm{~mm}$. An example of the contours in an image is given in Figure ID.

Pharmacokinetic parameters were estimated using the Patlak model (I7) on a voxelwise basis as previously described (8) with a phase-based population averaged vascular input function that was previously determined in the carotid artery (16). Shortly, CM concentrations in the plaque were determined from the signal intensity time course by using the Ernst equation and literature values for the longitudinal and transversal relaxation times of tissue (18) and the $r$, and $r_{2}$ relaxation rates of the CM (19). Resulting $\mathrm{K}^{\text {trans }}$ distribution was analyzed by calculating the mean, median and $75^{\text {th }}$ percentile. The median $95 \%$ confidence interval for $K^{\text {trans }}$ was determined to estimate uncertainty in parameter estimation.

\section{Statistical Analysis}

Differences in clinical characteristics between CEA and non-CEA patients were investigated using a Chi-square test (binary variables), independent samples T-test (continuous variables), or independent samples Kruskal-Wallis test (categorical variables), as appropriate. Mean, median, and $75^{\text {th }}$ percentile $K^{\text {trans }}$ values in two vessel wall regions (entire vessel wall versus adventitia) were compared using Pearson's correlation coefficient, a two-sided paired samples T-test, and Bland-Altman analysis. Mean, median, and $75^{\text {th }}$ percentile $K^{\text {trans }}$ from the entire vessel wall and adventitia were compared between CEA patients and non-CEA patients using an independent samples T-test. Association of mean, median, and $75^{\text {th }}$ percentile $\mathrm{K}^{\text {trans }}$ determined from the entire vessel wall and adventitial region with the relative microvessel density was 
assessed by calculation of Pearson's correlation coefficient. Correlation coefficients between 0.5 and 0.8 were considered moderately strong, while coefficients $>0.8$ were considered strong. All p-values below 0.05 were considered significant.

\section{RESULTS}

\section{Patient inclusion and characteristics}

In total, I I 8 patients were included. Eight patients could not be analyzed due to artifacts and/ or insufficient quality of the DCE-MR images. A total of 110 patients were used for analysis of DCE-MRI parameter agreement. Of these II 0 patients, 23 patients underwent CEA and obtained specimens were used for histological validation of DCE-MRI. Patient characteristics are displayed in Table I. The mean time interval between the last symptoms and DCE-MRI examination differed significantly between CEA and non-CEA patients $(20.9 \pm 5.2$ days versus

\section{Table I: Baseline characteristics of the included patients.}

Data are presented as mean \pm standard deviation or $n(\%)$. ${ }^{a}$ Data known for 108 out of 110 patients. Difference between CEA and non-CEA patients was investigated using a Chi-square test, independent samples T-test, or independent samples Kruskal-Wallis test, as appropriate.

\begin{tabular}{|c|c|c|c|c|c|}
\hline & & All patients & $\begin{array}{l}\text { CEA } \\
\text { patients }\end{array}$ & $\begin{array}{l}\text { Non-CEA } \\
\text { patients }\end{array}$ & P-value \\
\hline \multicolumn{2}{|l|}{ Number of patients [n] } & 110 & 23 & 87 & \\
\hline \multicolumn{2}{|l|}{ Age $[y]$} & $69 \pm 8.4$ & $65 \pm 8.2$ & $70 \pm 8.4$ & $p=0.013$ \\
\hline \multicolumn{2}{|l|}{ Male sex (\%) } & $75(68 \%)$ & $19(83 \%)$ & $56(64 \%)$ & $p=0.095$ \\
\hline \multicolumn{2}{|l|}{ Amaurosis fugax / TIA (\%) } & $68(62 \%)$ & $16(70 \%)$ & $52(60 \%)$ & $p=0.390$ \\
\hline \multicolumn{2}{|l|}{ Body mass index $\left[\mathrm{kg} / \mathrm{m}^{2}\right]$} & $26.8 \pm 4.2$ & $26.9 \pm 3.4$ & $26.8 \pm 3.7$ & $p=0.942$ \\
\hline \multicolumn{2}{|l|}{ Current smoking status (\%) } & $22(20 \%)$ & $6(26 \%)$ & $16(18 \%)$ & $p=0.412$ \\
\hline \multicolumn{2}{|l|}{ Diabetes mellitus (\%) } & $19(17 \%)$ & $5(22 \%)$ & $14(16 \%)$ & $p=0.524$ \\
\hline \multicolumn{2}{|l|}{ Hypertension (\%) } & $70(64 \%)$ & $20(87 \%)$ & $50(58 \%)$ & $p=0.009$ \\
\hline \multicolumn{2}{|l|}{ Hypercholesterolemia (\%) } & $56(52 \%)$ & $18(82 \%)$ & $38(44 \%)$ & $p=0.002$ \\
\hline \multicolumn{2}{|l|}{ Prior statin use (\%) } & $62(56 \%)$ & $21(96 \%)$ & $4 \mathrm{I}(48 \%)$ & $p<0.001$ \\
\hline \multicolumn{2}{|c|}{ Time between event and MRI [d] } & $37.3 \pm 22.0$ & $20.9 \pm 24.9$ & $41.6 \pm 19.6$ & $p<0.001$ \\
\hline \multirow{3}{*}{$\begin{array}{l}\text { Carotid Stenosis (duplex } \\
\text { ultrasonography) }\end{array}$} & $<50 \%$ & 53 & 0 & 53 & $p<0.001$ \\
\hline & $50-69 \%$ & 34 & 2 & 32 & \\
\hline & $70-99 \%$ & 21 & 21 & 0 & \\
\hline
\end{tabular}

Table 2: Pharmacokinetic DCE-MRI parameters' agreement.

Parameter agreement of $\mathrm{K}^{\text {trans }}$ (mean \pm standard error) was investigated using Pearson's Rank Correlation coefficient and two-sided paired samples T-test in I 10 patients. *P-value of two-sided paired samples T-test.

\begin{tabular}{|c|c|c|c|c|}
\hline Parameter & Vessel wall & Adventitia & Pearson's $\rho$ & P-value* \\
\hline Mean $\mathrm{K}^{\text {trans }}\left[\mathrm{min}^{-1}\right]$ & $0.055 \pm 0.001$ & $0.057 \pm 0.002$ & $0.70(p<0.001)$ & 0.17 \\
\hline Median $\mathrm{K}^{\text {trans }}\left[\mathrm{min}^{-1}\right]$ & $0.037 \pm 0.001$ & $0.039 \pm 0.001$ & $0.66(p<0.00 I)$ & $<0.01$ \\
\hline $75^{\text {th }}$ Percentile $\mathrm{K}^{\text {trans }}\left[\mathrm{min}^{-1}\right]$ & $0.066 \pm 0.002$ & $0.068 \pm 0.002$ & $0.68(p<0.00 I)$ & 0.20 \\
\hline
\end{tabular}


$41.6 \pm 2.1 ; p<0.00 I)$. A significantly higher degree of stenosis on duplex ultrasonography, prevalence of hypertension, hypercholesterolemia, and statin use prior to the ischemic event was observed in CEA patients, while a significant higher age for patients not scheduled for CEA was observed. The interval between DCE-MRI and CEA was $<24$ hours.

\section{DCE-MRI}

Examples of DCE-MR images are given in Figure I. A moderately strong positive correlation (Table 2; $\mathrm{n}=110$ ) was found between $\mathrm{K}^{\text {trans }}$ parameters from the entire vessel wall and adventitia for all descriptive statistics (Pearson's $\rho$ correlation coefficient range between 0.66 and $0.70, p<0.00 I)$. Median adventitial $K^{\text {trans }}$ was $5 \%$ larger than median vessel wall $K^{\text {trans }}(p=0.003)$, while no significant differences were found for mean and $75^{\text {th }}$ percentile $K^{\text {trans }}($ Table $2 ; n=\mid 10)$. Bland-Altman plots showed no variation of agreement across the range of values between the two vascular regions (Figure 2). No significant difference in uncertainty of parameter estimation for vessel wall $K^{\text {trans }}$ and adventitial $K^{\text {trans }}$ was found $(p=0.48)$.

No significant differences were found between CEA and non-CEA patients for mean, median, and $75^{\text {th }}$ percentile $K^{\text {trans }}$ from the vessel wall and adventitia ( $p \geq 0.09$, Table 3$)$.

\section{Correlation with histology}

An example of a histological image of the microvasculature is shown in Figure 3. The blood perfusion-vessel permeability product $\mathrm{K}^{\text {trans }}$ determined from both the entire vessel wall (Pearson's $\rho$ between 0.59 and 0.65, $p<0.003$ ) and the adventitial (Pearson's $\rho 0.52$ to 0.64 , $\mathrm{P}<0.0 \mathrm{II}$ ) region showed similar moderately strong positive correlation coefficients with the plaque microvasculature in the histological specimens (Table 4).
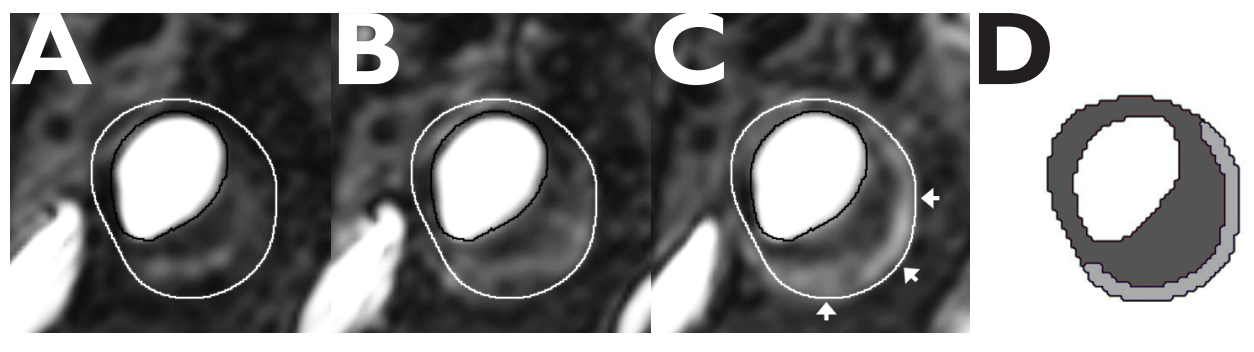

Figure I: Signal enhancement of the carotid artery on DCE-MR images.

DCE-MR images (A-C) from a patient with a severe stenosis of the internal carotid artery. The lumen boundary is shown in black, while the outer vessel wall is shown in white. Image A shows the carotid artery before contrast injection, while image $B$ and $C$ show the carotid artery $I$ and 6 minutes after contrast injection, respectively. The adventitial region (all pixels within $0.625 \mathrm{~mm}$ of the outer wall contour in a region of the vessel wall with a wall thickness > $1.5 \mathrm{~mm}$ ) clearly shows stronger signal enhancement compared to the remaining vessel wall tissue. This ring of enhancement is attributed to the vasa vasorum, which originate from the adventitia. The result of the automatic vessel segmentation is shown in image D. The adventitial region is depicted in light gray and the remaining vessel wall in dark gray. 
Table 3: Comparison of DCE-MRI parameters between CEA and non-CEA patients.

Values are presented as mean \pm standard error. ${ }^{~} P$-value of independent samples T-test.

\begin{tabular}{|c|c|c|c|c|}
\hline & & $\begin{array}{l}\text { CEA patients } \\
{[n=23]}\end{array}$ & $\begin{array}{l}\text { non-CEA } \\
\text { patients }[n=87]\end{array}$ & P-value \\
\hline \multirow{3}{*}{ Vessel wall $\mathrm{K}^{\text {trans }}$} & Mean $\left[\mathrm{min}^{-1}\right]$ & $0.05 I \pm 0.002$ & $0.057 \pm 0.002$ & 0.09 \\
\hline & Median $\left[\mathrm{min}^{-1}\right]$ & $0.035 \pm 0.002$ & $0.037 \pm 0.001$ & 0.21 \\
\hline & $75^{\text {th }}$ Percentile $\left[\mathrm{min}^{-1}\right]$ & $0.061 \pm 0.002$ & $0.067 \pm 0.002$ & 0.17 \\
\hline \multirow{3}{*}{ Adventitial wall $K^{\text {trans }}$} & Mean $\left[\mathrm{min}^{-1}\right]$ & $0.057 \pm 0.002$ & $0.057 \pm 0.002$ & 0.95 \\
\hline & Median $\left[\min ^{-1}\right]$ & $0.040 \pm 0.002$ & $0.039 \pm 0.001$ & 0.52 \\
\hline & $75^{\text {th }}$ Percentile $\left[\mathrm{min}^{-1}\right]$ & $0.069 \pm 0.003$ & $0.067 \pm 0.002$ & 0.68 \\
\hline
\end{tabular}

A

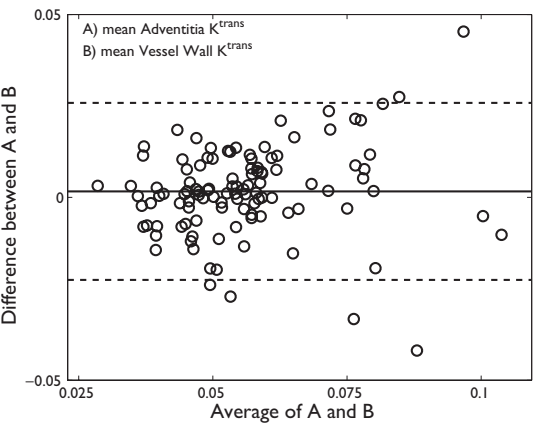

B

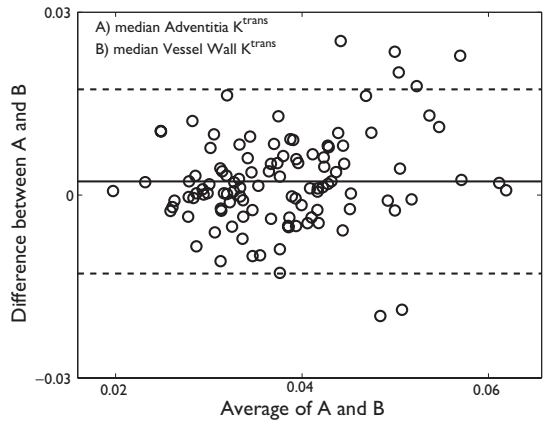

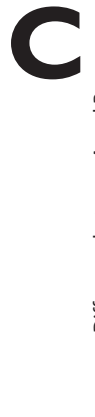

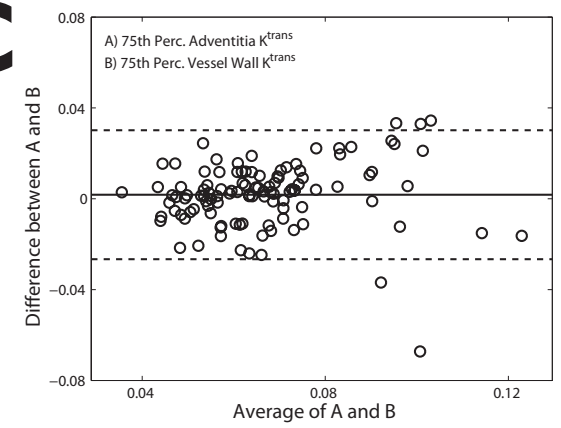

\section{Figure 2: Bland-Altman plots of different vascular regions.}

Bland Altman plots of mean (A), median (B), and $75^{\text {th }}$ percentile (C) $\mathrm{K}^{\text {trans }}$ values determined from the adventitia and entire vessel wall for patients with $\geq 2 \mathrm{~mm}$ carotid plaque. The mean difference is indicated by the black solid line and the $95 \%$ confidence interval of the mean difference by the dashed lines. Plots showed no variation of agreement across the range of values. 
Table 4: Correlation of DCE-MRI parameters with histology in 23 patients that underwent carotid endarterectomy.

Pearson's rank correlation coefficient (and corresponding P-values) of pharmacokinetic DCE-MRI parameters with the relative microvessel density (total CD3I-positive area divided by the total plaque area) as determined from carotid endarterectomy specimens.

\begin{tabular}{lll}
\hline & Vessel Wall Ktrans & Adventitial Ktrans \\
\hline Mean & $0.62(p<0.01)$ & $0.62(p<0.01)$ \\
Median & $0.59(p=0.01)$ & $0.52(p=0.01)$ \\
$75^{\text {th }}$ Percentile & $0.65(p=0.01)$ & $0.64(p<0.01)$ \\
\hline
\end{tabular}

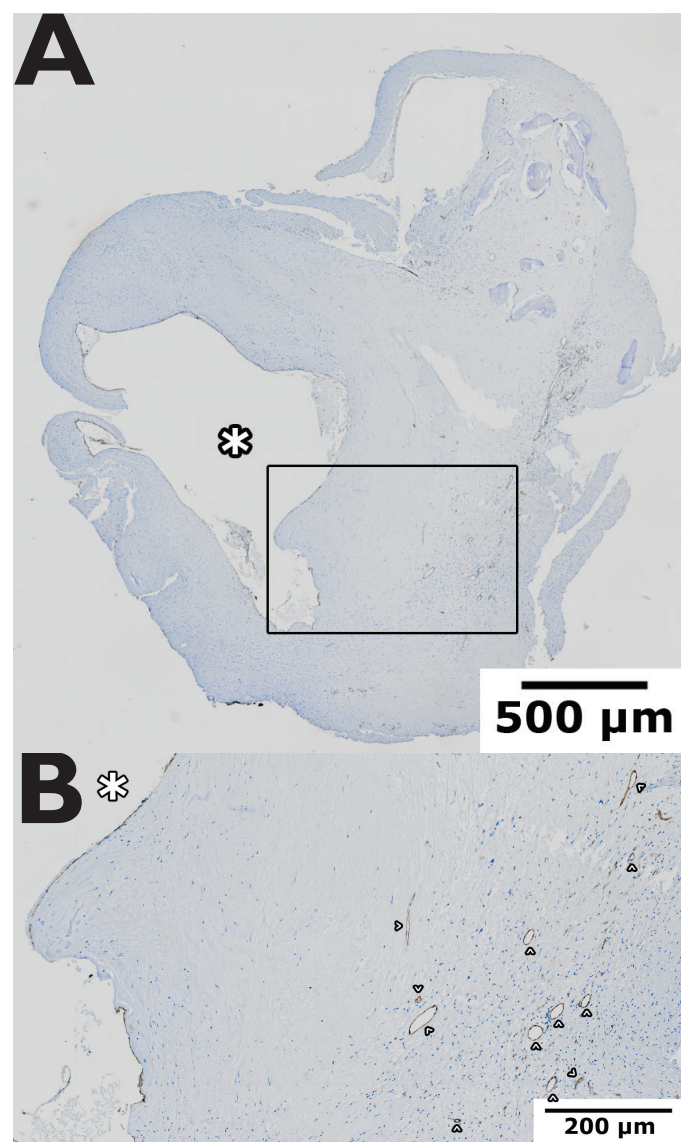

Figure 3: CD3 I immunohistochemistry of the atherosclerotic plaque microvasculature.

The microvessels can be observed in the CD3 I immunohistochemistry slice. An overview of the entire slice is given in panel $A$. The vessel lumen is indicated by the white asterisk. Close-up of microvessels of the region indicated by the box is given in panel $B$ and shows CD3I positive microvessels in brown that are indicated by arrow heads. 


\section{Discussion}

The present study showed that $\mathrm{K}^{\text {trans }}$ determined from two regions of the vascular wall (i.e. the entire vessel wall and the adventitia only) have similar moderately strong correlations with histological microvessel density in the plaque as a reference. A moderately strong positive correlation between vessel wall and adventitial $K^{\text {trans }}$ was found with a significantly higher median adventitial $\mathrm{K}^{\text {trans }}$ compared to median vessel wall $\mathrm{K}^{\text {trans }}$. Bland-Altman plots did not show variation of agreement across the range of values. Therefore, we showed that DCE-MRI parameters of both the entire vessel wall, as well as the adventitial region can be representative for the microvessel density of the symptomatic atherosclerotic plaque.

We observed a slightly higher value of median $\mathrm{K}^{\text {trans }}$ in the adventitial region compared to the entire vessel wall. A higher median value in the adventitial region is in line with the concept that plaque microvessels originate from this region and expand with disease progression $(5,20)$. In contrast, no significant differences between these two regions were found for mean and $75^{\text {th }}$ percentile $\mathrm{K}^{\text {trans }}$. The $75^{\text {th }}$ percentile $\mathrm{K}^{\text {trans }}$ may be more representative for microvascular hotspots, which will most likely be predominantly present in the adventitial part of the entire vessel wall. The mean $\mathrm{K}^{\text {trans }}$ was closer to the $75^{\text {th }}$ percentile value than the median value and may thus also have been significantly influenced by hotspots. Therefore, the mean and $75^{\text {th }}$ percentile $K^{\text {trans }}$ from the entire vessel wall might be more representative for the adventitial part of the entire vessel wall.

Based on the moderately strong positive correlations between different $\mathrm{K}^{\text {trans }}$ parameters, we can conclude that similar results regarding the association of DCE-MRI with either histology, other imaging modalities, or clinical parameters can be expected, regardless of the $\mathrm{K}^{\text {trans }}$ descriptive that is being reported. However, care should be taken when directly comparing absolute $\mathrm{K}^{\text {trans }}$ values between different studies, since the median adventitial $\mathrm{K}^{\text {trans }}$ is significantly higher than that of the vessel wall. For the determination of appropriate thresholds that can be used in clinical studies, investigators need to agree upon standardization of parameters.

Previously, it was demonstrated that symptomatic patients with a higher degree of carotid stenosis have a more vulnerable plaque phenotype (i.e. higher prevalence of intraplaque haemorrhage and a thin and/or ruptured fibrous cap and a larger lipid-rich necrotic core percentage and smaller fibrous tissue percentage) (2I). In contrast, in the present study, we did not find significant differences in $\mathrm{K}^{\text {trans }}$ values between patients that were scheduled for CEA (i.e. with a significant higher degree of carotid stenosis) and patients only receiving best medical treatment. Therefore, plaque microvasculature may already be increased in an earlier stage of the disease, which is in line with previous results from atherosclerotic plaques of the coronary arteries (22).

The correlation between histological measurements of plaque microvasculature and DCE$\mathrm{MRI}$ of carotid atherosclerosis of various regions of the vascular wall has been investigated in other studies. Kerwin et al. showed a strong positive correlation between vessel wall $\mathrm{K}^{\text {trans }}$ with the microvessel endothelium density on histology $(6,7)$. In addition, Gaens et al. showed a strong positive correlation between mean vessel wall $\mathrm{K}^{\text {trans }}$ and the mean endothelial microves- 
sel density on histology in 13 patients (8). A moderate correlation with relative microvessel cross-sectional area between plaque microvasculature and adventitial $K^{\text {trans }}$ was reported in a study of 25 patients (9). Correlation coefficients with histology of all descriptive statistics in the present study were thus comparable and within the range of previously reported studies (6-9).

A limitation of our study is the absence of the adventitia in the carotid plaque specimens obtained during CEA. Since only the intimal region containing the plaque and part of the media are surgically removed during CEA, the correlation of the microvessel density of the adventitia with $\mathrm{K}^{\text {trans }}$ cannot be investigated. The similar correlations of entire vessel wall and adventitial $\mathrm{K}^{\text {trans }}$ with measurements of the plaque microvasculature on histology and the moderately strong correlation between $\mathrm{K}^{\text {trans }}$ parameters, suggest that the microvasculature in the plaque is closely related to that in the surrounding adventitia, in line with previous results from autopsy studies of coronary plaques (22). A true validation of adventitial $\mathrm{K}^{\text {trans }}$ can only be performed in autopsy studies. Another limitation of our study is that only patients with a recent ischemic cerebrovascular event were included. Therefore, in our study we were unable to relate DCE-MRI findings to clinical symptoms. Last, because of the cross-sectional study design, we could not investigate the value of DCE-MRI to predict stroke. To investigate the value of DCE-MRI for risk stratification of patients, a longitudinal study is warranted.

In conclusion, our current study showed a moderately strong positive correlation for mean, median, and $75^{\text {th }}$ percentile $K^{\text {trans }}$ determined from the entire vessel wall and adventitial region with the relative microvessel density in histological specimens as a reference standard. This showed that both vascular regions can be representative for plaque microvessel density in symptomatic patients with severe carotid stenosis. Moderately strong correlation coefficients of DCE-MRI parameters from the vessel wall and adventitia were observed, with larger median $\mathrm{K}^{\text {trans }}$ values for the adventitial region. Future studies incorporating thresholds for risk stratification using DCE-MRI need to agree upon standardization of parameters used for analysis of DCE-MRI data. 


\section{References}

I. Mozaffarian D, Benjamin EJ, Go AS, Arnett DK, Blaha MJ, Cushman M et al. Heart Disease and Stroke Statistics - 2015 Update: A Report from the American Heart Association. Circulation 2015; I3 :e29-e322.

2. Moreno PR, Purushothaman K-R, Sirol M, Levy AP, Fuster V. Neovascularization in Human Atherosclerosis. Circulation 2006; I I3:2245-2252.

3. Kumamoto M, Nakashima Y, Sueishi K. Intimal Neovascularization in Human Coronary Atherosclerosis: Its Origin and Pathophysiological Significance. Hum Pathol 1995;26:450-456.

4. Virmani R, Kolodgie FD, Burke AP, Finn AV, Gold HK, Tulenko TN et al. Atherosclerotic Plaque Progression and Vulnerability to Rupture: Angiogenesis as a Source of Intraplaque Hemorrhage. Arterioscler Thromb Vasc Biol 2005;25:2054-206I.

5. Sluimer JC, Daemen MJ. Novel Concepts in Atherogenesis: Angiogenesis and Hypoxia in Atherosclerosis. J Pathol 2009;218:7-29.

6. Kerwin W. Quantitative Magnetic Resonance Imaging Analysis of Neovasculature Volume in Carotid Atherosclerotic Plaque. Circulation 2003; 107:85I-856.

7. Kerwin WS, O’Brien KD, Ferguson MS, Polissar N, Hatsukami TS, Yuan C. Inflammation in Carotid Atherosclerotic Plaque: A Dynamic Contrast-Enhanced Mr Imaging Study I. Radiology 2006;24I:459-468.

8. Gaens ME, Backes WH, Rozel S, Lipperts M, Sanders SN, Jaspers K et al. Dynamic Contrast-Enhanced Mr Imaging of Carotid Atherosclerotic Plaque: Model Selection, Reproducibility, and Validation. Radiology 20I3;266:27I-279.

9. Kerwin WS, Oikawa M, Yuan C, Jarvik GP, Hatsukami TS. Mr Imaging of Adventitial Vasa Vasorum in Carotid Atherosclerosis. Magn Reson Med 2008;59:507-5।4.

10. Truijman MT, Kwee RM, van Hoof RH, Hermeling E, van Oostenbrugge RJ, Mess WH et al. Combined I8f-Fdg Pet-Ct and Dce-Mri to Assess Inflammation and Microvascularization in Atherosclerotic Plaques. Stroke 2013;44:3568-3570.

II. Calcagno C, Ramachandran S, Izquierdo-Garcia D, Mani V, Millon A, Rosenbaum D et al. The Complementary Roles of Dynamic Contrast-Enhanced Mri and I8f-Fluorodeoxyglucose Pet/Ct for Imaging of Carotid Atherosclerosis. European journal of nuclear medicine and molecular imaging 2013;40: I884- I893.

12. Wang J, Liu H, Sun J, Xue H, Xie L, Yu S et al. Varying Correlation between I8f-Fluorodeoxyglucose Positron Emission Tomography and Dynamic Contrast-Enhanced Mri in Carotid Atherosclerosis: Implications for Plaque Inflammation. Stroke 20।4;45: I842-I845.

13. Sun J, Song Y, Chen H, Kerwin WS, Hippe DS, Dong L et al. Adventitial Perfusion and Intraplaque Hemorrhage: A Dynamic Contrast-Enhanced Mri Study in the Carotid Artery. Stroke 2013;44:1031-1036.

14. O'Brien KD, Hippe DS, Chen H, Neradilek MB, Probstfield JL, Peck S et al. Longer Duration of Statin Therapy Is Associated with Decreased Carotid Plaque Vascularity by Magnetic Resonance Imaging. Atherosclerosis 2015;245:74-8I.

15. Truijman MT, Kooi ME, van Dijk AC, de Rotte AA, van der Kolk AG, Liem MI et al. Plaque at Risk (Parisk): Prospective Multicenter Study to Improve Diagnosis of High-Risk Carotid Plaques. International journal of stroke : official journal of the International Stroke Society 20I4;9:747-754.

16. van Hoof RH, Hermeling E, Truijman MT, van Oostenbrugge RJ, Daemen JW, van der Geest RJ et al. Phase-Based Vascular Input Function: Improved Quantitative Dce-Mri of Atherosclerotic Plaques. Med Phys 2015;42:46I 9.

17. Patlak CS, Blasberg RG, Fenstermacher JD. Graphical Evaluation of Blood-to-Brain Transfer Constants from Multiple-Time Uptake Data. J Cereb Blood Flow Metab 1983;3:I-7.

18. Stanisz GJ, Odrobina EE, Pun J, Escaravage M, Graham SJ, Bronskill MJ et al. TI, T2 Relaxation and Magnetization Transfer in Tissue at 3t. Magn Reson Med 2005;54:507-5 I2.

19. Pintaske J, Martirosian P, Graf H, Erb G, Lodemann KP, Claussen CD et al. Relaxivity of Gadopentetate Dimeglumine (Magnevist), Gadobutrol (Gadovist), and Gadobenate Dimeglumine (Multihance) in Human Blood Plasma at 0.2, I.5, and 3 Tesla. Invest Radiol 2006;41:213-221. 
20. Sluimer JC, Gasc JM, van Wanroij JL, Kisters N, Groeneweg M, Sollewijn Gelpke MD et al. Hypoxia, HypoxiaInducible Transcription Factor, and Macrophages in Human Atherosclerotic Plaques Are Correlated with Intraplaque Angiogenesis. J Am Coll Cardiol 2008;5।:1258-1265. 


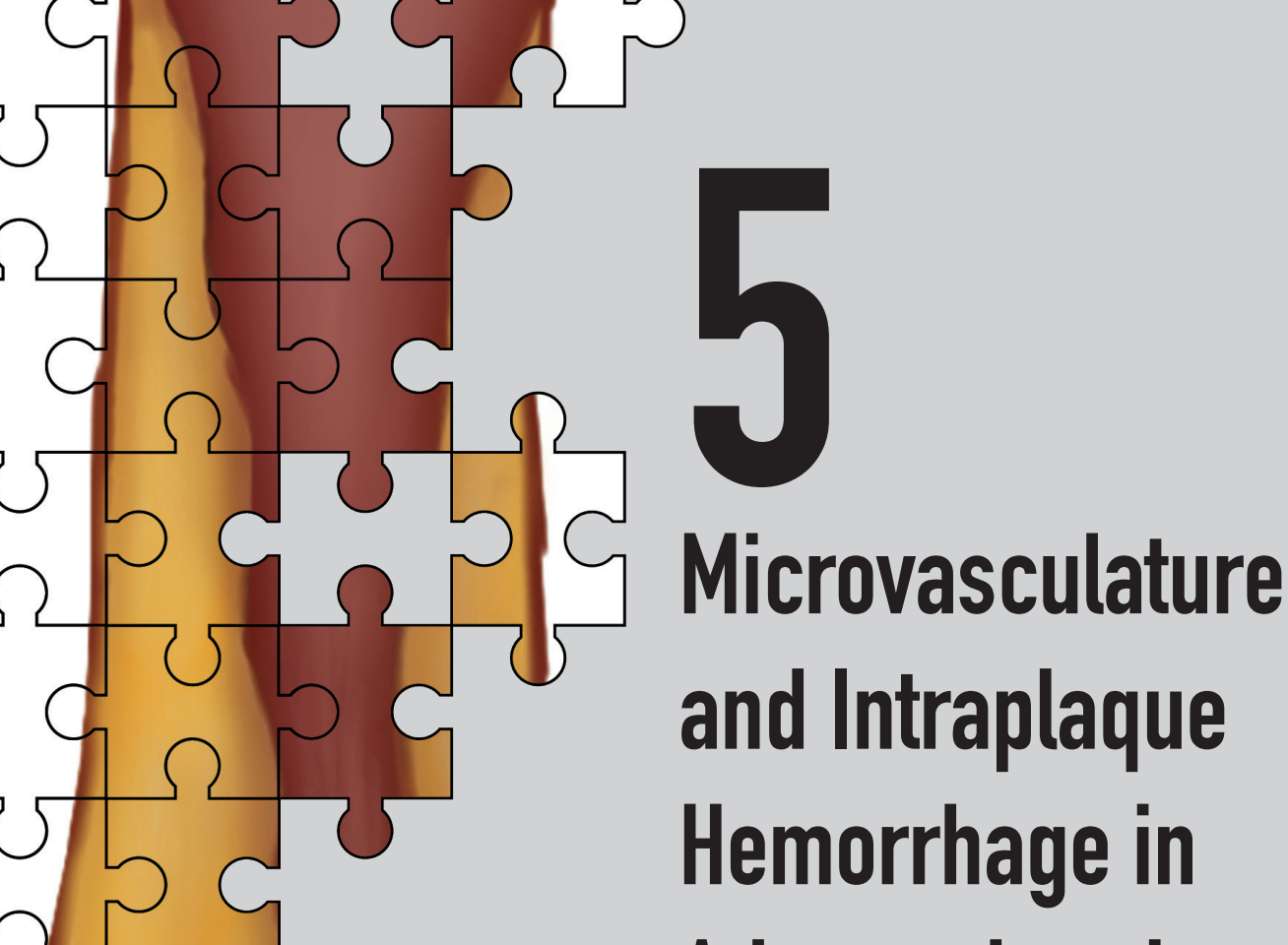

and Intraplaque

Hemorrhage in

Atherosclerotic

Carotid Lesions: an

MR Imaging Study

Raf H.M. van Hoof, Floris H.B.M. Schreuder, Martine

T.B Truijman, Nicky J.A. Wijnen, Sylvia Heeneman, Paul J.

Nederkoorn, Werner. H. Mess, Robert J. van Oostenbrugge,

Jan-Willem H. Daemen, Mat. J.A.P. Daemen, Joachim E.

Wildberger, M. Eline Kooi 


\section{ABSTRACT}

\section{Objectives}

The aim of the present study was to investigate whether there is a positive association between atherosclerotic plaque microvasculature and presence of intraplaque haemorrhage (IPH) in a relatively large prospective cohort study of patients with symptomatic carotid plaque.

\section{Background}

Presence of IPH on MRI strongly predicts cerebrovascular events. It is frequently suggested that IPH is caused by leakage of erythrocytes from immature microvessels, but the mechanisms leading to IPH are not fully understood.

\section{Methods}

IOI symptomatic patients with $\geq 2 \mathrm{~mm}$ carotid plaque underwent MRI of the symptomatic carotid plaque for detection of IPH and dynamic contrast-enhanced (DCE)-MRI for assessment of plaque microvasculature. $\mathrm{K}^{\text {trans }}$, an indicator of microvascular flow, density and leakiness, was estimated using pharmacokinetic modeling in the vessel wall and adventitia. Statistical analysis was performed using an independent samples T-test and logistic regression, correcting for clinical risk factors.

\section{Results}

A decreased vessel wall $\mathrm{K}^{\text {trans }}$ was found for IPH positive patients $\left(0.033 \pm 0.001 \mathrm{~min}^{-1}\right.$ versus $0.040 \pm 0.001, p=0.001$ ), which remained significant after correction for clinical risk factors associated with IPH or $\mathrm{K}^{\text {trans }}$. No difference in adventitial $\mathrm{K}^{\text {trans }}$ was found in patients with and without IPH $\left(0.040 \pm 0.002 \mathrm{~min}^{-1}\right.$ versus $\left.0.039 \pm 0.00 \mathrm{I}\right)$.

\section{Conclusions}

A reduced vessel wall $\mathrm{K}^{\text {trans }}$ is associated with the presence of $\mathrm{IPH}$, independent of clinical risk factors. Thus, we could not confirm a positive association between plaque microvasculature and IPH. Not only leaky plaque microvessels, but additional factors may contribute to IPH development. 


\section{INTRODUCTION}

Rupture of a vulnerable atherosclerotic plaque is very likely to be an important underlying cause of clinical ischemic events, such as stroke or myocardial infarction. Recent studies (I-7) have shown that the presence of intraplaque haemorrhage (IPH) is associated with plaque progression and predicts cerebrovascular events. The predictive value of carotid intraplaque hemorrhage for cerebrovascular events was confirmed in several meta-analyses (8-10), showing a hazard ratio of 5.7 (95\% confidence interval of 3.0 to 10.9) (8). Little is known about the pathophysiological origin of $\mathrm{IPH}$, although plaque microvasculature is generally considered to play an important role (I I). In general, these microvessels, originating from the adventitia, have poorly formed endothelial cell junctions (12). Therefore, it has been frequently suggested that IPH originates from leakage of erythrocytes from the plaque microvasculature into the plaque tissue (12).

MRI has been established as the preferred imaging method for the in vivo non-invasive detection of IPH within the atherosclerotic plaque (I3). In addition, dynamic contrast-enhanced (DCE)-MRI, using a low molecular weight non-specific Gadolinium-based contrast medium, has emerged as a noninvasive method to assess the plaque microvasculature of the carotid artery. Quantitative pharmacokinetic DCE-MRI parameters, in particular K ${ }^{\text {trans }}$ (a reflection of the microvessel flow, density, and permeability), have shown to correlate with the extent of plaque microvasculature determined using immunohistochemistry by us and others (14-17). In addition, it was shown that $\mathrm{K}^{\text {trans }}$ can be determined with a good inter-scan reproducibility (ICC of 0.79 , $p<0.05$ and coefficient of variation of $16 \%$ for the Patlak model) (I7). Since MRI allows non-invasive assessment of IPH and plaque microvasculature, it enables to explore the hypothesis of erythrocyte leakage from microvessels. Compared to the previously performed histopathological studies, $\mathrm{K}^{\text {trans }}$ is determined not only by microvessel density, but also by plaque microvasculature flow and leakiness. Moreover, MRI allows to study patients with a mild to moderate stenosis, who rarely undergo carotid endarterectomy and are therefore not available for histopathological studies. A recent MRI study ( 8 ) revealed a positive association between adventitial $\mathrm{K}^{\text {trans }}$ and the presence of $\mathrm{IPH}$ within a group of 27 symptomatic patients with carotid plaque and varying degree of stenosis. These findings are in line with the prevailing hypothesis that IPH originates from leaky plaque microvasculature. However, confirmation of these results in a larger patient group is needed for more definitive establishment of this association.

The aim of the present study was to further investigate the potential positive association between the atherosclerotic plaque microvasculature and presence of $\mathrm{PH}$ in a relatively large prospective cohort study in symptomatic patients with $\geq 2 \mathrm{~mm}$ carotid plaque. 


\section{Material and Methods}

\section{Study Population}

In the present study, consecutive symptomatic patients with a recent ischemic stroke/transient ischemic attack ( $<3$ months) and a carotid plaque $\geq 2 \mathrm{~mm}$ were eligible for inclusion in a prospective, observational imaging study. Patient demographics and clinical risk factors were collected during the study. Patients with a probable cardiac source of embolism, clotting disorder, severe comorbidity, and standard contra-indications for MRI, such as ferromagnetic/ other electronic implants were excluded. Patients with severe renal disease (creatinine clearance $<30 \mathrm{ml} / \mathrm{min}$ ) were not eligible for contrast-enhanced $M R I$ and therefore excluded from the current analysis. Approval of the local Institutional Ethical Review Board was obtained and written informed consent was obtained for all patients. The study was registered at ClinicalTrials.gov under NCTO 208025 and NCTO 1709045.

\section{MR imaging}

MR imaging was performed on a 3T whole body MRI system (Achieva, Philips Healthcare, Best, The Netherlands) using a dedicated 8-channel carotid RF coil (Shanghai Chenguan Medical Technologies Co., Shanghai, China). A previously described multi-contrast MR protocol (19) was used, which included the following sequences: 3D time-of-flight (TOF), 2D TI weighted (TIw) inversion recovery turbo field echo (IR-TFE), $T_{2}$ weighted (T2w) turbo spin echo (TSE) and pre-and post-contrast TI w double/quadruple inversion recovery (DIR/QIR) TSE. The acquired and reconstructed resolution was $0.62 \mathrm{~mm} \times 0.62-0.67 \mathrm{~mm}$ and $0.30 \mathrm{~mm} \times$ 0.24-0.30 mm, respectively. For DCE-MRI, an end diastolic ECG-gated 3D TI-TFE MRI pulse sequence was acquired centered at the position of the highest plaque burden with the following parameters: repetition/echo time $1 \mathrm{I} .6 / 5.7 \mathrm{~ms}$, flip angle $35^{\circ}$, Field of View $130 \times 130$

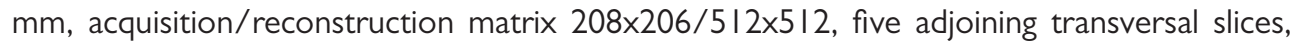
slice thickness $2 \mathrm{~mm}$ (20). The temporal resolution was approximately 20 seconds per time frame (dependent on heart rate). At the beginning of the third time frame, $0.1 \mathrm{mmol} / \mathrm{kg}$ of the small molecular contrast medium, Gadobutrol (Gadovist, Bayer HealthCare, Berlin, Germany), was injected as contrast medium (CM) with a power injector (Spectris Solaris, Medrad, Warrendale, PA, USA) at $0.5 \mathrm{ml} / \mathrm{sec}$ followed by a $20 \mathrm{ml}$ saline chaser at the same rate. DCEMRI acquisition was continued for six minutes after contrast injection. DCE-MR imaging was continued for six minutes after contrast injection.

\section{MR Image Review}

Plaque contours were drawn by a single experienced observer as described previously using dedicated vessel wall analysis software (VesselMASS, Leiden, the Netherlands) delineating the ipsilateral symptomatic carotid plaque (20). In case of doubt, a second highly experienced observer was consulted. Both readers were blinded for clinical characteristics and DCE-MRI test results. Per slice, luminal contours were drawn on the 3D TOF MR images, pre-contrast TIw QIR TSE, post-contrast TIw QIR TSE, or T2w TSE MR images, in subsequent order. Outer vessel wall contours were drawn by using the pre-contrast TI w QIR TSE, post-contrast TIw QIR TSE, TIw IR-TFE or T2w TSE MR images, in subsequent order. Normalized wall index (NWI), an indicator of plaque burden, was calculated as total vessel wall area divided by the sum of the luminal and vessel wall area. IPH was scored present by trained readers if a 
hyperintense signal (compared with the adjacent sternocleidomastoid muscle) was observed on the TIw TFE or TOF images in the bulk of the plaque.

\section{DCE-MR Image Review}

Luminal and outer vessel wall contours were transferred to the DCE-MR images and if necessary, contours were manually adjusted. The images acquired at each individual time frame were inspected and these images were shifted to correct for small patient displacements during the dynamic acquisition, if necessary. To avoid partial volume effects, luminal contours were corrected by keeping sufficient distance from the vessel lumen. When the adventitial vasa vasorum showed hyperenhancement after contrast material administration, outer plaque contours were corrected to include the adventitial vasa vasorum. The entire vessel wall region is defined as the region between the luminal and outer wall contours. The adventitial region of the vessel wall was delineated according to previously described criteria (18), i.e. all pixels within $0.625 \mathrm{~mm}$ of the outer wall contour in a region of the vessel wall with plaque (defined as having a wall thickness larger than $1.5 \mathrm{~mm}$ ).

\section{Data Analysis: Pharmacokinetic Modeling}

Pharmacokinetic parameters were estimated using the Patlak model (2I) on a voxel-wise basis as previously described (17) with a phase-based population averaged vascular input function determined in the carotid artery (20). Shortly, CM concentrations in the plaque were calculated from the signal intensity time course by using the Ernst equation based on literature values for the longitudinal and transversal relaxation times of tissue (22) and the $r_{1}$ and $r_{2}$ relaxation rates of CM (23) similar as in previous studies $(17,18,24,25)$.

\section{Statistical Analysis}

The associations between the presence of IPH, plaque burden (measured as NWI), and clinical risk factors was investigated using logistic regression with generalized linear mixed models. A similar procedure was followed for the association between NWI and clinical risk factors on the one hand and median vessel wall and median adventitial $\mathrm{K}^{\text {trans }}$ on the other hand using linear mixed models. To investigate the association between the presence of IPH and plaque perfusion (determined as vessel wall and adventitial $\mathrm{K}^{\text {trans }}$ ), binary logistic regression was performed. Clinical risk factors that were significantly associated with either DCE-MRI parameters for plaque perfusion or IPH were included in the binary logistic regression analysis. All P-values below 0.05 were considered statistically significant. Data are presented as mean \pm standard error or percentage. 


\section{RESULTS}

\section{Patient Characteristics}

Within the present study, a total of 124 patients were enrolled. Two patients did not receive injection of the contrast medium, and in fourteen patients, DCE-MRI was aborted due to ECG arrhythmias. Prior to analysis, seven additional patients were excluded due to insufficient MR image quality. Hence, we could analyze the association between the plaque microvasculature and IPH in I0I patients (67 male, age 70.3 \pm 8.5 years). Clinical characteristics of these patients are presented in Table I.

\section{Association between clinical characteristics and plaque burden with presence of IPH}

IPH was detected in 34 (34\%) patients. Of the clinical characteristics and plaque burden (Table 2), male sex $(O R=3.35,95 \% \mathrm{Cl}$ : I.23-9. I7; $p=0.02)$, hypertension $(O R=2.70,95 \% \mathrm{Cl}$ : I. I0 6.63; $p=0.03)$, and $\mathrm{NWI}(\mathrm{OR}=3.96,95 \% \mathrm{Cl}$ : 2.07-7.58; $\mathrm{p}<0.0 \mathrm{I})$ showed a significant positive association with the presence of IPH. After adjustment for these clinical risk factors in a binary logistic model, NWI remained significantly associated with the presence of IPH $(O R=3.52$, 95\% Cl: I.83-6.8I; p<0.01).

\section{Association between DCE-MRI model parameters and presence of IPH}

Representative DCE-MR images are presented in Figure I. No correlation was found between vessel wall $\mathrm{K}^{\text {trans }}$ and adventitial $\mathrm{K}^{\text {trans }}$ and the time from the most recent cerebrovascular ischemic event (Pearson $\rho=0.13, p=0.2 \mathrm{I}$ for vessel wall $K^{\text {trans }}$ and Pearson $\rho=0.04, p=0.70$ for adventitial $\left.\mathrm{K}^{\text {trans }}\right)$. Decreased median $\mathrm{K}^{\text {trans }}$ of the entire vessel wall was found for patients with $\mathrm{IPH}\left(0.033 \pm 0.00 \mathrm{I} \mathrm{min}^{-1}\right)$ compared to patients without IPH $\left(0.040 \pm 0.00 \mathrm{I} \mathrm{min}^{-1}\right.$, independent samples T-test $\mathrm{p}<0.0 \mathrm{I}$ ) (Figure 2). No difference was found for median adventitial $\mathrm{K}^{\text {trans }}$ in plaques with and without IPH $\left(0.040 \pm 0.002 \mathrm{~min}^{-1}\right.$ and $0.039 \pm 0.001 \mathrm{~min}-\mathrm{I}$, respectively). NWI was negatively associated with median $\mathrm{K}^{\text {trans }}$ from the entire vessel wall $(\Delta=-0.25, \mathrm{p}=0.0 \mathrm{I})$, but not with adventitial $K^{\text {trans }}(\Delta=0.00, p=0.99)$. Vessel wall $K^{\text {trans }}$ did show a significant negative association with the presence of IPH $(\mathrm{OR}=0.39,95 \% \mathrm{Cl}$ : $0.22-0.7 \mathrm{I} ; \mathrm{p}<0.0 \mathrm{I})$, while this was not the case for adventitial $\mathrm{K}^{\text {trans }}(\mathrm{OR}=\mathrm{I} . \mathrm{I} \mathrm{I}, 95 \% \mathrm{Cl}$ : 0.72- I.72; $\mathrm{p}=0.64)$.

After adjustment for clinical risk factors associated with the presence of IPH or $\mathrm{K}^{\text {trans }}$, vessel wall $K^{\text {trans }}(O R=0.46,95 \% \mathrm{Cl}: 0.24-0.89 ; p=0.02)$ remained negatively associated and $\mathrm{NWI}$ $(\mathrm{OR}=3.5 \mathrm{I}, 95 \% \mathrm{Cl}: \mathrm{I} .8 \mathrm{I}-6.79 ; \mathrm{P}<0.0 \mathrm{I})$ remained positively associated with the presence of $\mathrm{IPH}$. 


\section{Table I: Patient characteristics}

Data are presented as mean \pm standard deviation or $\mathrm{n}(\%)$. ${ }^{\mathrm{a}}$ Data known for 100 out of $10 \mathrm{I}$ patients.

\begin{tabular}{ll}
\hline Subjects [n] (\%) & I0I ( I 00\%) \\
\hline Age [y] & $70.3 \pm 8.5$ \\
\hline Male sex [n] (\%) & $67(66 \%)$ \\
\hline Body mass index [kg/m²] & $26.7 \pm 3.9$ \\
\hline Currently smokinga [n] (\%) & $19(19 \%)$ \\
\hline Diabetes mellitus [n] (\%) & $18(18 \%)$ \\
\hline Hypertension [n] (\%) & $58(57 \%)$ \\
\hline Hypercholesterolemia ${ }^{\mathrm{a}}[\mathrm{n}](\%)$ & $45(45 \%)$ \\
\hline Statin use before most recent CVEa $[\mathrm{n}](\%)$ & $49(49 \%)$ \\
\hline Time between event and MRI [days] & $39 \pm 22$ \\
\hline
\end{tabular}

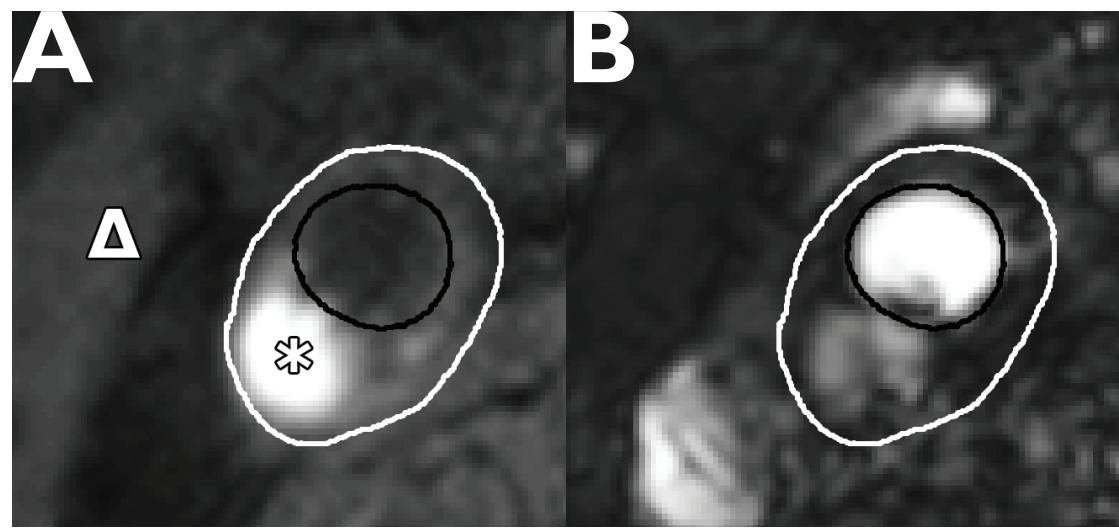

Figure I: MR images from a patient with a severe stenosis of the internal carotid artery.

The black line delineates the lumen boundary, while the white line delineates the outer vessel wall. Image A shows a T I w IR-TFE image with a hyperintense signal within the bulk the plaque compared with the adjacent sternocleidomastoid muscle $(\Delta)$, indicating the presence of intraplaque hemorrhage (asterisk). Image B shows a DCE-MR images before contrast injection. 

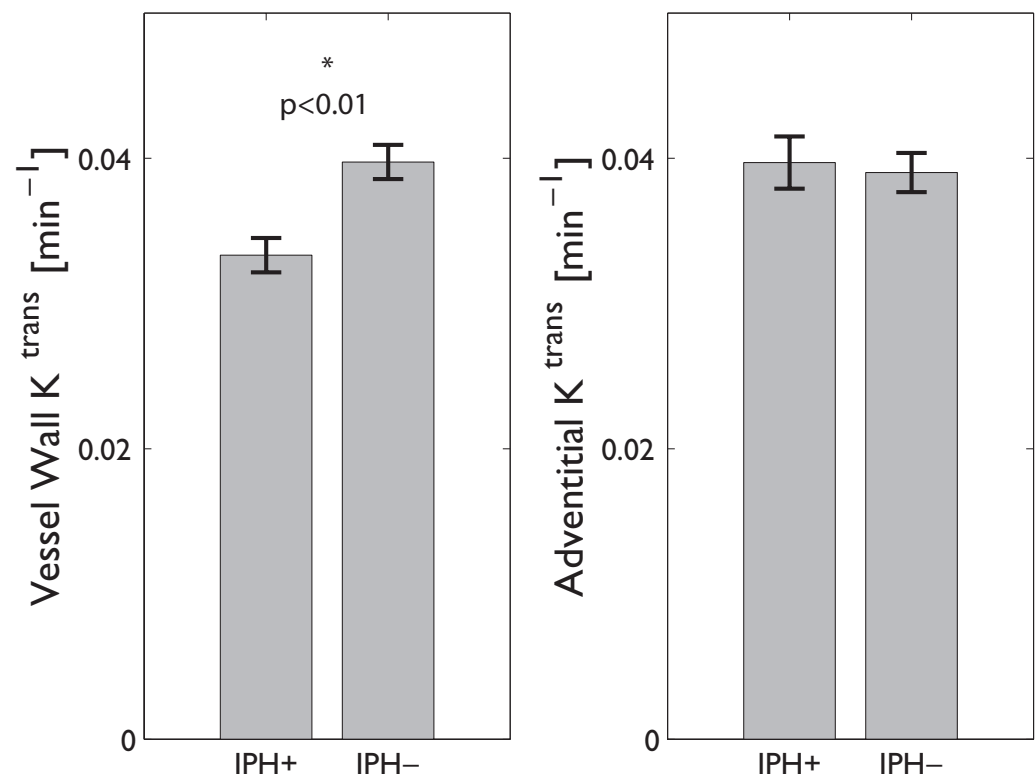

Figure 2: Vessel wall and adventitial $K^{\text {trans }}$ versus IPH status

Vessel wall and adventitial $\mathrm{K}^{\text {trans }}$ (mean \pm standard error) for patients with $(\mathrm{IPH}+$ ) and without intraplaque haemorrhage (IPH-).

\section{Table 2: Association between clinical characteristics and plaque burden versus presence of IPH}

$\mathrm{P}$-value of univariate analysis between clinical risk factors and intraplaque haemorrhage (IPH) and median $\mathrm{K}^{\text {trans }}$ determined from the entire vessel wall (VW) or the adventitia. For IPH, association was tested using binary logistic regression and reported using odds ratio with 95\% confidence interval. Linear mixed models were used to assess association of the continuous parameter $\mathrm{K}^{\text {trans }}$ with clinical risk factors.

\begin{tabular}{|c|c|c|c|}
\hline Clinical risk factor & IPH & median VW $\mathbf{K}^{\text {trans }}$ & $\begin{array}{l}\text { median } \\
\text { adventitial } \mathbf{K}^{\text {trans }}\end{array}$ \\
\hline Age, per 5 years & $1.17(0.90-1.52 ; p=0.25)$ & $\Delta=0.07(p=0.20)$ & $\Delta=0.11(p=0.06)$ \\
\hline Male sex & $3.35(1.23-9.17 ; p=0.02)$ & $\Delta=-0.08(p=0.69)$ & $\Delta=0.07(p=0.72)$ \\
\hline Body mass index & $0.68(0.42-1.11 ; p=0.13)$ & $\Delta=0.07(p=0.5 \mathrm{I})$ & $\Delta=0.00(p=0.98)$ \\
\hline Currently smoking & $0.45(0.14-1.49 ; p=0.20)$ & $\Delta=0.09(p=0.72)$ & $\Delta=-0.02(p=0.93)$ \\
\hline Diabetes mellitus & $1.32(0.46-3.78 ; p=0.61)$ & $\Delta=-0.34(p=0.17)$ & $\Delta=-0.25(p=0.30)$ \\
\hline Hypertension & $2.70(1.10-6.63 ; p=0.03)$ & $\Delta=0.09(p=0.66)$ & $\Delta=0.12(p=0.5 \mathrm{I})$ \\
\hline Hypercholesterolemia & $1.36(0.59-3.12 ; p=0.47)$ & $\Delta=-0.08(p=0.66)$ & $\Delta=-0.11(p=0.55)$ \\
\hline Statin use before most recent CVE & $1.16(0.51-2.68 ; p=0.72)$ & $\Delta=-0.02(p=0.93)$ & $\Delta=-0.09(p=0.65)$ \\
\hline Plaque burden (NWI), per I SD & 3.96 (2.07-7.58; $p<0.01)$ & $\Delta=-0.25(p=0.01)$ & $\Delta=0.00(p=0.99)$ \\
\hline
\end{tabular}




\section{Discussion}

In the present prospective imaging study in symptomatic patients, no difference in adventitial $\mathrm{K}^{\text {trans }}$, indicative for microvascular density, flow and/or permeability, was found in carotid plaques with and without IPH. We could thus not confirm a positive association between (leaky) microvasculature $\left(\mathrm{K}^{\text {trans }}\right)$ and $\mathrm{IPH}$.

Several previous histopathological $(26,27)$ studies did report a link between IPH and microvessel density, although this link was not present in all vascular beds and it was also demonstrated that plaques with IPH with a low microvascular density are quite common. Derksen et al. (26) reported significantly higher percentage of plaques with increased microvessel density in the presence of $\mathrm{PH}$ (50\% versus 42\%) in 752 carotid endarterectomy specimens, however no difference was found in 209 femoral endarterectomy plaque specimens. Derksen et al. also demonstrated that $50 \%$ of the carotid plaques with IPH had a low microvascular density, showing that IPH also occurs frequently in plaques with low microvascular density. McCarthy et al. (27) reported significantly more microvessels in plaques with IPH in a study of 28 carotid endarterectomy specimens, but did not report quantitative data on the increase in plaque microvasculature. Gössl et al. (28) showed in hearts of 15 patients, obtained at autopsy, a positive Kendall-Tau beta rank correlation between microvessel density in the coronary vessel wall and glycophorin A score (indicative for hemorrhage) of $0.65(\mathrm{p}<0.0 \mathrm{I})$. Note that also normal vessel wall segments with typically a low glycophorin A score and a low microvasculature density were included in their data analysis, which may have affected the correlation. $A$ difference between histopathological and DCE-MRI studies, is that in histological studies the microvascular density is measured, while the DCE-MRI parameter $\mathrm{K}^{\text {trans }}$ does not only reflect the microvascular density, but also microvascular flow and leakiness.

In a previous DCE-MRI study, an increased adventitial $\mathrm{K}^{\text {trans }}$ was found in the presence of IPH in 27 patients with symptomatic carotid plaque (18). Twelve of these patients demonstrated $\mathrm{IPH}$ at the symptomatic side, while 4 patients demonstrated IPH at the contralateral, asymptomatic side. We could not confirm this positive association between adventitial $\mathrm{K}^{\text {trans }}$ and IPH, despite our relatively large number of patients with $\geq 2 \mathrm{~mm}$ carotid plaque that were included in the present prospective study. A difference between our study and that by Sun et al. (18) is that Sun et al. studied the maximum value of mean $\mathrm{K}^{\text {trans }}$ across all slices, which represents microvascular hotspots in the adventitia. A sub-analysis of the $75^{\text {th }}$ percentile value of the $K^{\text {trans }}$ distribution in the adventitial region in the present study, which is also indicative for hotspots of leaky microvessels, revealed no significant differences in plaques with and without IPH. In addition, we found lower median $\mathrm{K}^{\text {trans }}$ values in the entire vessel wall in patients with IPH. The lower values of $\mathrm{K}^{\text {trans }}$ in the entire vessel wall may be due to an increased amount of necrotic tissue in plaques with IPH. Indeed a significant, negative Pearson's correlation coefficient ( $\rho=-$ $0.26, \mathrm{p}<0.0 \mathrm{I}$ ) was found for median vessel wall $\mathrm{K}^{\text {trans }}$ with the fraction of the lipid-rich necrotic core (LRNC) volume with respect to the entire vessel wall volume. These results indicate a decrease of the atherosclerotic plaque microvasculature in plaques with a larger LRNC. 
The prevailing hypothesis is that IPH originates from leakage of erythrocytes out of immature microvessels into the plaque tissue (12). In the present study, we could not confirm a positive association between leaky plaque microvessels and IPH. The present findings suggest that leakage of erythrocytes is not increased in plaques with IPH several weeks after a cerebrovascular event. Additional processes may thus also contribute to IPH. It has already been suggested that disruptions of the fibrous cap (e.g. plaque fissures or plaque rupture) can lead to IPH (29). A histopathological study (30) showed that fibrous cap fissures were frequently accompanied by $\mathrm{IPH}$. In line with the concept that IPH can also originate from fibrous cap disruption, it was recently shown that the presence of intraplaque haemorrhage is associated with a disruption of the atherosclerotic plaque surface (plaque ulceration and/or a fissured fibrous cap) in patients with a mild to moderate carotid stenosis (3I).

Plaque biomechanics may be an important factor in the formation of IPH, which is supported by several findings in literature. First, one recent study in 80 asymptomatic subjects with a 16 $79 \%$ carotid stenosis showed that a low diastolic blood pressure was associated with IPH (32). Additionally, pulse pressure, which may be considered as driving force for plaque deformation during the cardiac cycle, has been identified as a strong determinant of IPH (33). Second, it has been shown histologically that IPH occurs more frequently in the upstream region of the plaque (34), where the blood pressure is much higher, due to pressure wave reflection. Third, computational models showed that IPH is associated with higher structural wall stress (35).

Next to the decrease in vessel wall $\mathrm{K}^{\text {trans }}$, a positive association between $\mathrm{NWI}$ (indicative for plaque burden) and presence of IPH was found in the current study. This is in line with previous work, since it was already demonstrated that $\mathrm{IPH}$ is associated with wall thickness and plaque length (32) and other studies indicated that IPH promotes plaque progression (I-4).

Recent research (36) in 98 patients from the AIM-HIGH Trial (37) has shown an inverse relationship between the duration that patients were on statin therapy and plaque microvasculature fraction $\left(v_{p}\right)$, suggesting that plaque microvasculature may be affected by statin use, possibly due to their anti-inflammatory properties (38). No association between $\mathrm{K}^{\text {trans }}$ and the use of statins before the most recent cerebrovascular event was found in the present study. However, our study may have been underpowered to find such a relation, since only 49 patients used statins before their most recent cerebrovascular event. Immediately after the event, statins were prescribed in the majority (90\%) of the patients. The MRI examinations was performed $39 \pm 22$ days after the ischemic event.

Due to the relatively small size of carotid plaques, a high spatial resolution of DCE-MRI is crucial, limiting the temporal resolution. Accurate determination of the first pass peak of the VIF is not possible with the high spatial resolution MRI pulse sequence. Therefore, within the current study a previously determined population averaged vascular input function was used, similar as in previous studies $(17,20)$. 
Development of the plaque microvasculature is a dynamic process, which is thought to be related to inflammation in the plaque tissue $(24,39,40)$. Increased activity of macrophages within the plaque tissue is associated with hypoxia and subsequent angiogenic stimuli $(41,42)$ can result in the formation of new microvessels. Previous research has shown that the presence of IPH does not change significantly over a period of up to I.5 years $(3,4,43)$. This suggest that $\mathrm{IPH}$ is either a continuous process or haemoglobin, which produces the hyperintense signal on IR-TFE MRI, is entrapped within the atherosclerotic plaque for a long time period. The findings of the present study indicate that there is no continuous leakage of erythrocytes from plaque microvasculature several weeks after an ischemic event, since we found no increase in $\mathrm{K}^{\text {trans }}$ in plaques with IPH at this time point. The development of plaque microvasculature over time still needs to be determined in future studies. It cannot be excluded that IPH and the resulting healing process can lead to a reduction in plaque microvasculature.

\section{Conclusions}

We have found a negative association between IPH and vessel wall $\mathrm{K}^{\text {trans }}$, indicative for microvascular flow, density, and leakiness. These results indicate that no ongoing leakage of erythrocytes from plaque microvasculature occurs several weeks after a cerebrovascular event in plaques with IPH. Thus, not only leaky microvessels, but also additional factors, such as disrupted plaque surface, may contribute to the development of IPH. Further longitudinal studies are warranted to unravel underlying mechanisms contributing to IPH. 


\section{REFERENCES}

I. Kolodgie FD, Gold HK, Burke AP, Fowler DR, Kruth HS, Weber DK et al. Intraplaque Hemorrhage and Progression of Coronary Atheroma. N Engl J Med 2003;349:23 I6-2325.

2. Sun J, Underhill HR, Hippe DS, Xue Y, Yuan C, Hatsukami TS. Sustained Acceleration in Carotid Atherosclerotic Plaque Progression with Intraplaque Hemorrhage: A Long-Term Time Course Study. JACC Cardiovasc Imaging 2012;5:798-804.

3. Takaya N, Yuan C, Chu B, Saam T, Polissar NL, Jarvik GP et al. Presence of Intraplaque Hemorrhage Stimulates Progression of Carotid Atherosclerotic Plaques: A High-Resolution Magnetic Resonance Imaging Study. Circulation 2005; I I I:2768-2775.

4. Underhill HR, Yuan C, Yarnykh VL, Chu B, Oikawa M, Polissar NL et al. Arterial Remodeling in [Corrected] Subclinical Carotid Artery Disease. JACC Cardiovasc Imaging 2009;2: I38I - 1389.

5. Kwee RM, van Oostenbrugge RJ, Mess WH, Prins MH, van der Geest RJ, ter Berg JWM et al. Mri of Carotid Atherosclerosis to Identify Tia and Stroke Patients Who Are at Risk of a Recurrence. Journal of Magnetic Resonance Imaging 2012; 10.1002/jmri.23918:n/a-n/a.

6. Turc G, Oppenheim C, Naggara O, Eker OF, Calvet D, Lacour JC et al. Relationships between Recent Intraplaque Hemorrhage and Stroke Risk Factors in Patients with Carotid Stenosis: The Hirisc Study. Arterioscler Thromb Vasc Biol 2012;32:492-499.

7. Hellings WE, Peeters W, Moll FL, Piers SR, van Setten J, Van der Spek PJ et al. Composition of Carotid Atherosclerotic Plaque Is Associated with Cardiovascular Outcome: A Prognostic Study. Circulation 2010;121:1941-1950.

8. Saam T, Hetterich H, Hoffmann V, Yuan C, Dichgans M, Poppert $\mathrm{H}$ et al. Meta-Analysis and Systematic Review of the Predictive Value of Carotid Plaque Hemorrhage on Cerebrovascular Events by Magnetic Resonance Imaging. J Am Coll Cardiol 2013;62:1081-1091.

9. Hosseini AA, Kandiyil N, Macsweeney ST, Altaf N, Auer DP. Carotid Plaque Hemorrhage on Magnetic Resonance Imaging Strongly Predicts Recurrent Ischemia and Stroke. Ann Neurol 2013;73:774-784.

10. Gupta A, Baradaran H, Schweitzer AD, Kamel H, Pandya A, Delgado D et al. Carotid Plaque Mri and Stroke Risk: A Systematic Review and Meta-Analysis. Stroke 2013;44:307I-3077.

II. Moreno PR, Purushothaman K-R, Sirol M, Levy AP, Fuster V. Neovascularization in Human Atherosclerosis. Circulation 2006; I 13:2245-2252.

12. Virmani R, Kolodgie FD, Burke AP, Finn AV, Gold HK, Tulenko TN et al. Atherosclerotic Plaque Progression and Vulnerability to Rupture: Angiogenesis as a Source of Intraplaque Hemorrhage. Arterioscler Thromb Vasc Biol 2005;25:2054-2061.

13. Cappendijk VC, Cleutjens KB, Heeneman S, Schurink GW, Welten RJ, Kessels AG et al. In Vivo Detection of Hemorrhage in Human Atherosclerotic Plaques with Magnetic Resonance Imaging. J Magn Reson Imaging 2004:20: 105-110.

14. Kerwin WS, Oikawa M, Yuan C, Jarvik GP, Hatsukami TS. Mr Imaging of Adventitial Vasa Vasorum in Carotid Atherosclerosis. Magn Reson Med 2008;59:507-5।4.

15. Kerwin WS, O'Brien KD, Ferguson MS, Polissar N, Hatsukami TS, Yuan C. Inflammation in Carotid Atherosclerotic Plaque: A Dynamic Contrast-Enhanced Mr Imaging Studyl. Radiology 2006;24I:459-468.

16. Kerwin W. Quantitative Magnetic Resonance Imaging Analysis of Neovasculature Volume in Carotid Atherosclerotic Plaque. Circulation 2003; 107:85 I-856.

17. Gaens ME, Backes WH, Rozel S, Lipperts M, Sanders SN, Jaspers K et al. Dynamic Contrast-Enhanced Mr Imaging of Carotid Atherosclerotic Plaque: Model Selection, Reproducibility, and Validation. Radiology 2013;266:27I-279.

18. Sun J, Song Y, Chen H, Kerwin WS, Hippe DS, Dong L et al. Adventitial Perfusion and Intraplaque Hemorrhage: A Dynamic Contrast-Enhanced Mri Study in the Carotid Artery. Stroke 2013;44:I03 I- 1036. 
19. Truijman MT, Kooi ME, van Dijk AC, de Rotte AA, van der Kolk AG, Liem MI et al. Plaque at Risk (Parisk): Prospective Multicenter Study to Improve Diagnosis of High-Risk Carotid Plaques. International journal of stroke : official journal of the International Stroke Society 20।4;9:747-754.

20. van Hoof RH, Hermeling E, Truijman MT, van Oostenbrugge RJ, Daemen JW, van der Geest RJ et al. Phase-Based Vascular Input Function: Improved Quantitative Dce-Mri of Atherosclerotic Plaques. Med Phys 20I 5;42:4619.

2I. Patlak CS, Blasberg RG, Fenstermacher JD. Graphical Evaluation of Blood-to-Brain Transfer Constants from Multiple-Time Uptake Data. J Cereb Blood Flow Metab 1983;3:I-7.

22. Stanisz GJ, Odrobina EE, Pun J, Escaravage M, Graham SJ, Bronskill MJ et al. TI, T2 Relaxation and Magnetization Transfer in Tissue at 3t. Magn Reson Med 2005;54:507-5 I 2.

23. Pintaske J, Martirosian P, Graf H, Erb G, Lodemann KP, Claussen CD et al. Relaxivity of Gadopentetate Dimeglumine (Magnevist), Gadobutrol (Gadovist), and Gadobenate Dimeglumine (Multihance) in Human Blood Plasma at 0.2, I.5, and 3 Tesla. Invest Radiol 2006;41:2I3-22I.

24. Truijman MT, Kwee RM, van Hoof RH, Hermeling E, van Oostenbrugge RJ, Mess WH et al. Combined I8f-Fdg Pet-Ct and Dce-Mri to Assess Inflammation and Microvascularization in Atherosclerotic Plaques. Stroke 2013;44:3568-3570.

25. Kerwin WS, Liu F, Yarnykh V, Underhill H, Oikawa M, Yu W et al. Signal Features of the Atherosclerotic Plaque at 3.0 Tesla Versus I.5 Tesla: Impact on Automatic Classification. J Magn Reson Imaging 2008;28:987-995.

26. Derksen WJ, Peeters W, van Lammeren GW, Tersteeg C, de Vries JP, de Kleijn DP et al. Different Stages of Intraplaque Hemorrhage Are Associated with Different Plaque Phenotypes: A Large Histopathological Study in 794 Carotid and 276 Femoral Endarterectomy Specimens. Atherosclerosis 201 I;21 8:369-377.

27. McCarthy MJ, Loftus IM, Thompson MM, Jones L, London NJ, Bell PR et al. Angiogenesis and the Atherosclerotic Carotid Plaque: An Association between Symptomatology and Plaque Morphology. J Vasc Surg 1999;30:26I268.

28. Gossl M, Versari D, Hildebrandt HA, Bajanowski T, Sangiorgi G, Erbel R et al. Segmental Heterogeneity of Vasa Vasorum Neovascularization in Human Coronary Atherosclerosis. JACC Cardiovasc Imaging 20 10;3:32-40.

29. Falk E. Plaque Rupture with Severe Pre-Existing Stenosis Precipitating Coronary Thrombosis. Characteristics of Coronary Atherosclerotic Plaques Underlying Fatal Occlusive Thrombi. British heart journal 1983;50: I27-I34.

30. Constantinides P. Plaque Fissures in Human Coronary Thrombosis. Journal of Atherosclerosis Research 1966;6:1-17.

31. van Dijk AC, Truijman MT, Hussain B, Zadi T, Saiedie G, de Rotte AA et al. Intraplaque Hemorrhage and the Plaque Surface in Carotid Atherosclerosis: The Plaque at Risk Study (Parisk). AJNR Am J Neuroradiol 2015;36:2127-2133.

32. Sun J, Canton G, Balu N, Hippe DS, Xu D, Liu J et al. Blood Pressure Is a Major Modifiable Risk Factor Implicated in Pathogenesis of Intraplaque Hemorrhage: An in Vivo Magnetic Resonance Imaging Study. Arterioscler Thromb Vasc Biol 2016; 10.1 161/atvbaha. I 15.307043.

33. Selwaness M, van den Bouwhuijsen QJ, Verwoert GC, Dehghan A, Mattace-Raso FU, Vernooij M et al. Blood Pressure Parameters and Carotid Intraplaque Hemorrhage as Measured by Magnetic Resonance Imaging: The Rotterdam Study. Hypertension 2013;61:76-81.

34. Cicha I, Worner A, Urschel K, Beronov K, Goppelt-Struebe M, Verhoeven E et al. Carotid Plaque Vulnerability: A Positive Feedback between Hemodynamic and Biochemical Mechanisms. Stroke 201 I;42:3502-35 I0.

35. Huang X, Teng Z, Canton G, Ferguson M, Yuan C, Tang D. Intraplaque Hemorrhage Is Associated with Higher Structural Stresses in Human Atherosclerotic Plaques: An in Vivo Mri-Based 3d Fluid-Structure Interaction Study. Biomedical engineering online 2010;9:86.

36. O'Brien KD, Hippe DS, Chen H, Neradilek MB, Probstfield JL, Peck S et al. Longer Duration of Statin Therapy Is Associated with Decreased Carotid Plaque Vascularity by Magnetic Resonance Imaging. Atherosclerosis 20। 5;245:74-8I. 
37. Investigators A-H, Boden WE, Probstfield JL, Anderson T, Chaitman BR, Desvignes-Nickens P et al. Niacin in Patients with Low Hdl Cholesterol Levels Receiving Intensive Statin Therapy. N Engl J Med 20 I I;365:2255-2267.

38. Jain MK, Ridker PM. Anti-Inflammatory Effects of Statins: Clinical Evidence and Basic Mechanisms. Nat Rev Drug Discov 2005;4:977-987.

39. Calcagno C, Ramachandran S, Izquierdo-Garcia D, Mani V, Millon A, Rosenbaum D et al. The Complementary Roles of Dynamic Contrast-Enhanced Mri and I8f-Fluorodeoxyglucose Pet/Ct for Imaging of Carotid Atherosclerosis. European journal of nuclear medicine and molecular imaging 20। 3;40: I 884- I893.

40. Wang J, Liu H, Sun J, Xue H, Xie L, Yu S et al. Varying Correlation between I8f-Fluorodeoxyglucose Positron Emission Tomography and Dynamic Contrast-Enhanced Mri in Carotid Atherosclerosis: Implications for Plaque Inflammation. Stroke 2014;45: I842- I845.

4I. Sluimer JC, Gasc JM, van Wanroij JL, Kisters N, Groeneweg M, Sollewijn Gelpke MD et al. Hypoxia, HypoxiaInducible Transcription Factor, and Macrophages in Human Atherosclerotic Plaques Are Correlated with Intraplaque Angiogenesis. J Am Coll Cardiol 2008;5 I:I258-I265.

42. Sluimer JC, Daemen MJ. Novel Concepts in Atherogenesis: Angiogenesis and Hypoxia in Atherosclerosis. J Pathol 2009;2 18:7-29.

43. Kwee RM, Truijman MTB, van Oostenbrugge RJ, Mess WH, Prins MH, Franke CL et al. Longitudinal Mri Study on the Natural History of Carotid Artery Plaques in Symptomatic Patients. PLoS ONE 2012;7:e42472.Patlak CS, Blasberg RG, Fenstermacher JD. Graphical Evaluation of Blood-to-Brain Transfer Constants from Multiple-Time Uptake Data. J Cereb Blood Flow Metab 1983;3:I-7. 


\section{Abstract}

\section{Background and Purpose}

Hallmarks of vulnerable atherosclerotic plaques are inflammation that can be assessed with I8fluorine-fluorodeoxyglucose positron emission tomography/computed tomography, and increased neovascularization that can be evaluated by dynamic contrast-enhanced-MRI. It remains unclear whether these parameters are correlated or represent independent imaging parameters. This study determines whether there is a correlation between inflammation and neovascularization in atherosclerotic carotid plaques.

\section{Methods}

A total of 58 patients with transient ischemic attack or minor stroke in the carotid territory and ipsilateral carotid artery stenosis of $30 \%$ to $69 \%$ were included. All patients underwent positron emission tomography/computed tomography and dynamic contrast-enhanced-MRI of the carotid plaque. ${ }^{18}$ Fluorine-fluorodeoxyglucose standard uptake values with target/ background ratio were determined. Neovascularization was quantified by the mean (leakage) volume transfer constant $\mathrm{K}^{\text {trans }}$. Spearman rank correlation coefficients between target/background ratio and $\mathrm{K}^{\text {trans }}$ were calculated.

\section{Results}

A total of 58 patients with transient ischemic attack or minor stroke in the carotid territory and ipsilateral carotid artery stenosis of $30 \%$ to $69 \%$ were included. All patients underwent positron emission tomography/computed tomography and dynamic contrast-enhanced-MRI of the carotid plaque. ${ }^{18}$ Fluorine-fluorodeoxyglucose standard uptake values with target/ background ratio were determined. Neovascularization was quantified by the mean (leakage) volume transfer constant $\mathrm{K}^{\text {trans }}$. Spearman rank correlation coefficients between target/background ratio and $\mathrm{K}^{\text {trans }}$ were calculated.

\section{Conclusions}

There is a weak but significant positive correlation between inflammation on positron emission tomography/computed tomography and neovascularization as assessed with dynamic contrast-enhanced-MRI. Future studies should investigate which imaging modality has the highest predictive value for recurrent stroke, as these are not interchangeable. 


\section{INTRODUCTION}

Stroke is the fourth leading cause of death in the United States and a leading cause of longterm disability. Atherosclerosis is underlying in the majority of clinical cardiovascular events, such as stroke (I). Inflammation is an important feature of plaque progression and vulnerability $(2,3)$. It can be quantified noninvasively with ${ }^{18}$ fluorine-fluorodeoxyglucose $\left({ }^{18} \mathrm{~F}-\mathrm{FDG}\right)$ positron emission tomography/computed tomography (PET-CT) with excellent reproducibility (4-6). Neovascularization is another important feature of vulnerable atherosclerotic plaques. Dynamic contrast-enhanced MRI (DCE-MRI) enables quantitative assessment of neovascularization in carotid atherosclerotic plaques (7-9). $K^{\text {trans }}$ is a parameter that reflects microvascular flow, permeability, and surface area (9). The aim of the present study was to investigate the relation between inflammation and neovascularization as assessed with PET-CT and DCEMRI, respectively. In a secondary analysis, we investigated the relation between these parameters and plaque morphology itself as determined with MRI. In addition, the relation between clinical characteristics, $\mathrm{K}^{\text {trans }}$ and ${ }^{18} \mathrm{~F}-\mathrm{FDG}$ uptake were evaluated. 


\section{Material and Methods}

\section{Patients}

Patients with recent ( $<3$ months) amaurosis fugax, transient ischemic attack or minor stroke in the carotid territory and ipsilateral carotid plaque causing a mild to moderate $(30 \%-69 \%)$ stenosis at Doppler ultrasonography (10), were eligible for inclusion. Cardiovascular risk factors and the use of medication were assessed by a questionnaire (I I). The study was approved by the institutional Medical Ethical Committee. All patients gave written informed consent.

\section{Imaging}

PET-CT examination and the multisequence MRI have in detail been described previously (I2). Parameters of the DCE-MRI protocol have been published as well (9).

\section{Image Analysis}

The analysis of the PET-CT images was performed using a dedicated workstation (I2). To determine the target/background ratio (TBR), the standard uptake values were normalized to blood ${ }^{18} \mathrm{~F}$-FDG activity by dividing them by the mean standard uptake value of blood as measured in the internal jugular vein (Figure). TBR is considered to be a reflection of arterial FDG uptake and reflective of underlying macrophage activity (13). Plaque morphology was manually assessed using dedicated vessel wall analysis software (VesselMASS; LUMC, The Netherlands) (12). The operator was blinded for the PET-CT results. Quantification of neovascularization was done using a custom-made Matlab program (Matlab version 7.5; The Mathworks, Natick, MA), using the Patlak model and a generalized vascular input function $(9, \mid 4)$. The mean $\mathrm{K}^{\text {trans }}$ per patient was determined. In addition, histogram analysis was performed to determine the $75^{\text {th }}$ percentile $\mathrm{K}^{\text {trans }}$, which is more reflective for hot spots with high $\mathrm{K}^{\text {trans }}$ values (Figure).

\section{Statistical Analysis}

To correlate TBR with $\mathrm{K}^{\text {trans }}$, Spearman rank correlation coefficient was calculated using SPSS version 20 (SPSS Inc, Chicago, IL). Relation between clinical characteristics, TBR and $\mathrm{K}^{\text {trans }}$, was explored by univariate linear regression analysis. A p-value $<0.05$ was considered statistically significant. 


\section{REsULTS}

In total 58 patients were included. Nine of them had to be excluded, because of poor DCE-

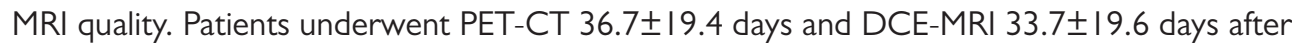
their last neurological symptoms. Mean time interval between both examinations was $2.9 \pm 5.5$ days. For the 49 patients analyzed, clinical characteristics and their relation between $\mathrm{K}^{\text {trans }}$ and ${ }^{18} \mathrm{~F}-\mathrm{FDG}$ are displayed in Table I. The mean TBR was calculated as I.45 \pm 0.26 . The morphological parameters of the plaque as determined with MRI are given in Table 2. The mean and $75^{\text {th }}$ percentile $K^{\text {trans }}$ were determined as $0.11 \pm 0.03$ and $0.15 \pm 0.04 \mathrm{~min}^{-1}$, respectively. A positive weak correlation between TBR and $\mathrm{K}^{\text {trans }}$ was found (mean $\mathrm{K}^{\text {trans }}$, Spearman $\rho$ of 0.30 $(p=0.035) ; 75^{\text {th }}$ percentile $K^{\text {trans }}$, Spearman $\rho$ of $\left.0.29(p=0.04 I)\right)$. No significant correlations between TBR and $\mathrm{K}^{\text {trans }}$ in relation to the morphological parameters were observed.

\section{Table I}

Cardiovascular risk factors of the 49 analyzed patients and the results of the univariate linear regression analysis with TBR and mean $\mathrm{K}^{\text {trans }}$.

\begin{tabular}{|c|c|c|c|c|c|c|c|}
\hline & & TBR & & & Mean $\mathbf{K}^{\text {trans }}$ & & \\
\hline Characteristics & No (\%) & $\begin{array}{l}\text { Standardized } \\
\text { Coefficient } \beta\end{array}$ & $95 \% \mathrm{Cl}$ & $\begin{array}{l}P \\
\text { Value }\end{array}$ & $\begin{array}{l}\text { Standardized } \\
\text { Coefficient } \beta\end{array}$ & $95 \% \mathrm{Cl}$ & $\begin{array}{l}\mathrm{P} \\
\text { Value }\end{array}$ \\
\hline Male & $29(59)$ & 0.13 & $-0.08-0.22$ & 0.87 & 0.12 & $-0.01-0.02$ & 0.39 \\
\hline Age, $y$, mean $\pm S D$ & $65.3 \pm 8.8$ & -0.02 & $-0.01-0.01$ & 0.91 & -0.19 & $-0.00-0.00$ & 0.20 \\
\hline \multicolumn{8}{|l|}{ Smoking } \\
\hline Current & $12(25)$ & -0.11 & $-0.24-0.11$ & 0.44 & 0.05 & $-0.02-0.02$ & 0.75 \\
\hline Former & $20(4 I)$ & & & & & & \\
\hline Never & $17(34)$ & -0.05 & $-0.18-0.13$ & 0.72 & -0.16 & $-0.03-0.01$ & 0.29 \\
\hline Hypertension & $42(86)$ & -0.30 & $-0.42--0.01$ & 0.04 & -0.13 & $-0.03-0.01$ & 0.37 \\
\hline Diabetes mellitus & II (22) & -0.16 & $-0.27-0.08$ & 0.26 & -0.10 & $-0.03-0.01$ & 0.50 \\
\hline $\begin{array}{l}\text { History of ischemic } \\
\text { heart disease }\end{array}$ & $8(16)$ & -0.23 & $-0.35-0.04$ & 0.12 & -0.09 & $-0.03-0.02$ & 0.55 \\
\hline $\begin{array}{l}\text { Use of statins } \\
\text { before event }\end{array}$ & $18(37)$ & -0.20 & $-0.26-0.05$ & 0.17 & 0.02 & $-0.02-0.02$ & 0.87 \\
\hline $\begin{array}{l}\text { Use of statins after } \\
\text { event }\end{array}$ & $46(94)$ & -0.22 & $-0.54-0.07$ & 0.12 & 0.10 & $-0.02-0.04$ & 0.49 \\
\hline
\end{tabular}



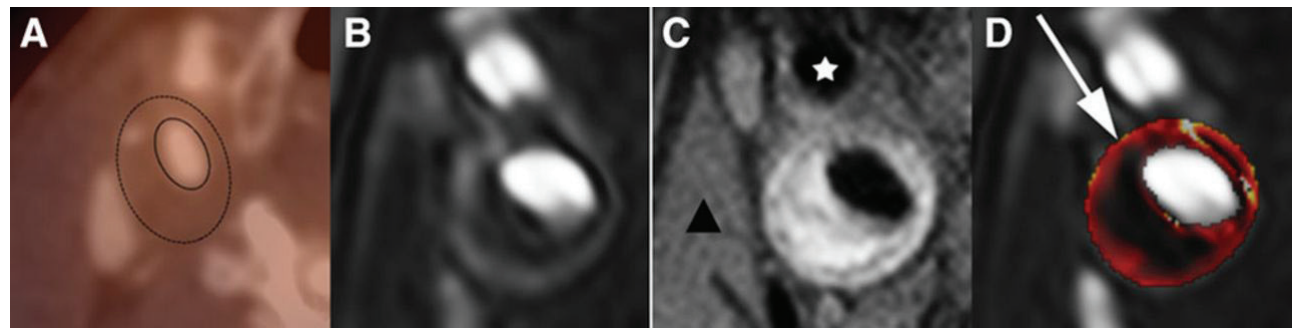

\section{Figure}

Positron emission tomography/computed tomography (PET-CT; A) and dynamic contrast-enhanced (DCE) MRI (B-D) of a transverse section of the carotid plaque from a 64-year-old man. A, Fused PET-CT image. The lumen is delineated by the black line. The plaque is delineated by the dashed black line. B, Six minutes after contrast injection. C, TI-weighted Turbo Spin Echo MRI. D, Parametric Krans map overlaid on DCE-MRI. Voxel Krans values are color encoded from 0 to $0.2 \mathrm{~min}^{-1}$. The lipid-rich necrotic core exhibits low $\mathrm{K}^{\text {trans }}$ values at the center of the plaque. The highly vascularized adventitia (high $\mathrm{K}^{\text {trans }}$ values) at the outer rim (arrow) is clearly visualized. $\star$, External carotid artery; and $\boldsymbol{\Delta}$, sternocleidoid muscle.

Table 2: Association between clinical characteristics and plaque burden versus presence of IPH

Correlations between target-to-background ratio (TBR), mean $\mathrm{K}^{\text {trans }}$, and plaque morphology as assessed on MRI.

\begin{tabular}{llllll}
\hline & & TBR & \multicolumn{3}{c}{ Mean K $\mathbf{K}^{\text {trans }}$} \\
\hline Parameter & Mean \pm SD & Spearman $\rho$ & P Value & Spearman $\rho$ & P Value \\
\hline Total lumen volume $\left[\mathrm{mm}^{3}\right]$ & $889.21 \pm 290.56$ & 0.04 & 0.70 & -0.03 & 0.82 \\
\hline Total wall volume $\left[\mathrm{mm}^{3}\right]$ & $979.84 \pm 333.31$ & 0.05 & 0.73 & -0.20 & 0.18 \\
\hline Total vessel volume $\left[\mathrm{mm}^{3}\right]$ & $1869.05 \pm 508.16$ & 0.04 & 0.78 & -0.09 & 0.55 \\
\hline Mean wall thickness $[\mathrm{mm}]$ & $1.46 \pm 0.42$ & 0.04 & 0.79 & -0.17 & 0.25 \\
\hline Max wall thickness $[\mathrm{mm}]$ & $3.99 \pm 1.430 .26$ & 0.07 & 0.05 & 0.73 & \\
\hline Normalized wall thickness & $0.52 \pm 0.09$ & 0.13 & 0.37 & -0.19 & 0.20 \\
\hline
\end{tabular}




\section{Discussion}

The purpose of this study was to investigate the relation between inflammation (PET-CT) and neovascularization (DCE-MRI) in carotid atherosclerotic plaques. We demonstrated that higher FDG uptake is weakly associated with increased microvasculature within the plaque. Cyran et al. (15) also showed positive correlation between the mean TBR and the extraction fraction of DCE-MRI. Both imaging modalities provide information about hallmarks of plaque vulnerability, but it is unknown whether these imaging features are interchangeable or provide additive information. This study proves that, in symptomatic patients with mild to moderate $(30 \%-69 \%)$ stenosis, PET-CT and DCE-MRI features of the plaque are related with each other, although the information provided is not interchangeable. This is expressed in the relative low correlation coefficient $(\rho=0.30 ; p<0.05)$. However, we have not studied the use of these techniques for treatment decision making. Further studies should determine the clinical usage of inflammation and neovascularization for the individual patient's management.

\section{Conclusions}

There is a weak but significant positive correlation between inflammation on PET-CT and neovascularization as assessed with DCE-MRI. Future studies should investigate that which imaging modality has the highest predictive value for recurrent stroke, as these are not interchangeable. 


\section{ReferenCes}

I. Go AS, Mozaffarian D, Roger VL, Benjamin EJ, Berry JD, Blaha MJ et al. Heart Disease and Stroke Statistics-2014 Update: A Report from the American Heart Association. Circulation 2013;10.1161/01. cir.000044II39.02102.80.

2. Libby P. Inflammation in Atherosclerosis. Nature 2002;420:868-874.

3. Ross R. Atherosclerosis--an Inflammatory Disease. N Engl J Med 1999;340: I I5-I26.

4. Rudd JH, Warburton EA, Fryer TD, Jones HA, Clark JC, Antoun N et al. Imaging Atherosclerotic Plaque Inflammation with [I8f]-Fluorodeoxyglucose Positron Emission Tomography. Circulation 2002; 105:2708-27II.

5. Tawakol A, Migrino RQ, Bashian GG, Bedri S, Vermylen D, Cury RC et al. In Vivo I8f-Fluorodeoxyglucose Positron Emission Tomography Imaging Provides a Noninvasive Measure of Carotid Plaque Inflammation in Patients. J Am Coll Cardiol 2006;48: I8I8-1824.

6. Rudd JH, Myers KS, Bansilal S, Machac J, Rafique A, Farkouh M et al. (I8)Fluorodeoxyglucose Positron Emission Tomography Imaging of Atherosclerotic Plaque Inflammation Is Highly Reproducible: Implications for Atherosclerosis Therapy Trials. J Am Coll Cardiol 2007;50:892-896.

7. Kerwin W. Quantitative Magnetic Resonance Imaging Analysis of Neovasculature Volume in Carotid Atherosclerotic Plaque. Circulation 2003; 107:85I-856.

8. Kerwin WS, O'Brien KD, Ferguson MS, Polissar N, Hatsukami TS, Yuan C. Inflammation in Carotid Atherosclerotic Plaque: A Dynamic Contrast-Enhanced Mr Imaging Study I. Radiology 2006;24I:459-468.

9. Gaens ME, Backes WH, Rozel S, Lipperts M, Sanders SN, Jaspers K et al. Dynamic Contrast-Enhanced Mr Imaging of Carotid Atherosclerotic Plaque: Model Selection, Reproducibility, and Validation. Radiology 20I3;266:27I-279.

10. Grant EG, Benson CB, Moneta GL, Alexandrov AV, Baker JD, Bluth El et al. Carotid Artery Stenosis: Grayscale and Doppler Ultrasound Diagnosis--Society of Radiologists in Ultrasound Consensus Conference. Ultrasound quarterly 2003;19:190-198.

II. Kwee RM, van Oostenbrugge RJ, Prins MH, Ter Berg JW, Franke CL, Korten AG et al. Symptomatic Patients with Mild and Moderate Carotid Stenosis: Plaque Features at Mri and Association with Cardiovascular Risk Factors and Statin Use. Stroke 2010;41:1389-1393.

12. Kwee RM, Teule GJ, van Oostenbrugge RJ, Mess WH, Prins MH, van der Geest RJ et al. Multimodality Imaging of Carotid Artery Plaques: I8f-Fluoro-2-Deoxyglucose Positron Emission Tomography, Computed Tomography, and Magnetic Resonance Imaging. Stroke 2009;40:37I8-3724.

13. Tawakol A, Migrino RQ, Hoffmann U, Abbara S, Houser S, Gewirtz H et al. Noninvasive in Vivo Measurement of Vascular Inflammation with F-18 Fluorodeoxyglucose Positron Emission Tomography. J Nucl Cardiol 2005; 12:294-301.

14. Patlak CS, Blasberg RG, Fenstermacher JD. Graphical Evaluation of Blood-to-Brain Transfer Constants from Multiple-Time Uptake Data. J Cereb Blood Flow Metab 1983;3:I-7.

15. Cyran CC, Sourbron S, Bochmann K, Habs M, Pfefferkorn T, Rominger A et al. Quantification of SupraAortic Arterial Wall Inflammation in Patients with Arteritis Using High Resolution Dynamic Contrast-Enhanced Magnetic Resonance Imaging: Initial Results in Correlation to [ I8f]-Fdg Pet/Ct. Invest Radiol 20 I I;46:594-599. 


\section{ABSTRACT}

\section{Background}

Patients with a recent ischemic stroke have a higher risk of recurrent stroke compared to (ocular) transient ischemic attack (TIA) patients. Plaque microvasculature is considered as a feature of plaque vulnerability and can be quantified with carotid dynamic contrast-enhanced magnetic resonance imaging (DCE-MRI). Purpose of this cross-sectional study was to explore the association between plaque microvasculature with type of recent cerebrovascular events in symptomatic patients with mild-to-moderate carotid stenosis.

\section{Methods}

87 symptomatic patients with a recent stroke $(n=35)$ or (ocular) TIA $(n=52)$ underwent carotid DCE-MRI examination. Plaque microvasculature was studied in the vessel wall and adventitia of symptomatic carotid plaques using dynamic contrast-enhanced (DCE)-MRI and the pharmacokinetic modeling parameter $\mathrm{K}^{\text {trans }}$. Statistical analysis was performed with logistic regression, correcting for associated clinical risk factors.

\section{Results}

$75^{\text {th }}$ percentile adventitial (odds ratio: $1.97, \mathrm{Cl}=\mathrm{I}$. I8-3.29) $\mathrm{K}^{\text {trans }}$ was significantly associated with a recent ischemic stroke compared to (ocular) TIA in multivariate analysis, while clinical risk factors were not significantly associated with the type of event.

\section{Conclusions}

This study indicates a positive association of leaky plaque microvasculature with a recent ischemic stroke compared to (ocular) TIA. Prospective longitudinal studies are needed to investigate whether $\mathrm{K}^{\text {trans }}$ may serve as an imaging marker to predict (the type of) future cerebrovascular events. 


\section{INTRODUCTION}

Annually 15 million people suffer from a stroke worldwide, from which 5 million people die and 5 million people are left permanently disabled (I). Ischemic strokes account for approximately $80 \%$ of all strokes. Rupture of a vulnerable atherosclerotic plaque of the carotid artery is an important underlying cause for ischemic stroke. Epidemiological studies have identified various clinical risk factors associated with an increased risk of ischemic stroke (2-7). Previous studies have also shown an higher risk for recurrent stroke in patients with a ischemic stroke compared to patients with a transient ischemic attack (TIA). The risk for a recurrent stroke within seven days was $5.2 \%$ after a TIA versus 1 I.5\% after a stroke (8). Additional research showed that patients with a minor stroke have an increased risk for a recurrent stroke compared to patients with a recent TIA or ocular TIA (also known as amaurosis fugax) (9). Data from the European Carotid Surgery Trial (ECST) trial also showed that the hazard ratio for a 5-year risk of ipsilateral ischemic stroke depends on the type of the presenting cerebrovascular event (10). The underlying mechanisms responsible for the differences in risk for a recurrent event between the stroke subtypes are unclear.

Increased microvasculature within the atherosclerotic plaque has been proposed as a marker of plaque vulnerability $(1 \mathrm{I}, 12)$. These microvessels grow from the adventitia into the plaque and generally have an impaired endothelial integrity, potentially providing an entry point for inflammatory cells and erythrocytes to the atherosclerotic lesion. It is believed that the growth of these microvessels into the plaque region is triggered by an increased activity of inflammatory cells, and subsequent hypoxia, within the plaque tissue (13).

Dynamic contrast-enhanced (DCE-)MRI has emerged as a non-invasive imaging technique to assess plaque microvasculature (14-16). With DCE-MRI, signal enhancement time curve after contrast medium (CM) injection is analyzed to quantify plaque microvasculature using pharmacokinetic modeling. We and others have shown that the volume transfer constant $\mathrm{K}^{\text {trans }}$ (a reflection of the microvascular density, flow, and permeability) correlates with the amount of microvessels within the plaque determined from histology (14-17) as a reference standard. We have also demonstrated a good interscan reproducibility for $\mathrm{K}^{\text {trans }}(16)$.

It is unknown whether $\mathrm{K}^{\text {trans }}$ is associated with the stroke subtype. Given the increased risk for recurrent stroke in stroke patients compared to (ocular) TIA patients, we hypothesized that plaque microvasculature might differ between these patients groups.

\section{Study aim}

The aim of the present cross-sectional observational study was to explore the association between leaky plaque microvasculature with the subtype of recent cerebrovascular symptoms (ischemic stroke versus (ocular) TIA) in patients with a symptomatic mild to moderate carotid artery stenosis. 


\section{Material and Methods}

\section{Study population}

The present study was performed as a substudy of the Plaque At RISK (PARISK) study( I8) (ClinicalTrials.gov unique identifier NCT0 208025). Consecutive symptomatic patients with a recent ( $<3$ months) minor ischemic stroke, or (ocular) TIA with a mild to moderate ipsilateral stenosis of the carotid artery were eligible for inclusion. A mild to moderate stenosis was defined as a plaque of at least $2 \mathrm{~mm}$ to carotid stenosis $<70 \%$ (based on NASCET criteria( (9)). For the present study, patients from a single center (Maastricht University Medical Center) underwent additional DCE-MRI and were included for current analysis. Exclusion criteria were standard contra-indications for MRI, such as ferromagnetic or other electronic implants. Patients with severe renal disease (creatinine clearance $<30 \mathrm{ml} /$ minute) were not eligible for contrast-enhanced MRI and were therefore excluded from the current analysis. Clinical history and medication use were ascertained at the time of subject enrolment. Approval of the local Institutional Ethical Review Board was obtained and all patients provided written informed before study inclusion.

\section{MR imaging}

MR imaging was performed on a 3T whole body MRI system (Achieva, Philips Healthcare, Best, The Netherlands) using a dedicated 8-channel carotid RF coil (Shanghai Chenguan Medical Technologies Co., Shanghai, China). A previously described multi-contrast MR protocol(I8) was used, which included the following sequences: 3D time-of-flight (TOF), 2D TI weighted (TIw) inversion recovery turbo field echo (IR-TFE), $T_{2}$ weighted (T2w) turbo spin echo (TSE) and pre- and post-contrast TI w double/quadruple inversion recovery (DIR/ QIR) TSE. Fifteen adjoining transversal slices with $2 \mathrm{~mm}$ slice thickness and an acquired and reconstructed resolution of $0.62 \times 0.62-0.67$ and $0.30 \times 0.24-0.30$, respectively, were acquired. For DCE-MRI, an end-diastolic ECG-gated 3D TI-TFE MRI pulse sequence, centered at the position of highest plaque burden, was acquired with the following parameters: repetition/ echo time II.6/5.7ms, flip angle $35^{\circ}$, Field of View $130 \times 130 \mathrm{~mm}$, acquisition and reconstruction matrix $208 \times 206$ and $512 \times 512$, respectively, five consecutive transversal slices, slice thickness $2 \mathrm{~mm}$.(20) The temporal resolution was approximately 20 seconds per time frame (dependent on heart rate). At the beginning of the third time frame, $0.1 \mathrm{mmol} / \mathrm{kg}$ of Gadobutrol (Gadovist, Bayer HealtCare, Berlin, Germany) was injected as contrast medium (CM) with a power injector (Spectris Solaris, Medrad, Warrendale, USA) at $0.5 \mathrm{ml} / \mathrm{sec}$ followed by a $20 \mathrm{ml}$ saline chaser at the same rate. DCE-MR imaging was continued for six minutes after contrast injection.

\section{MR Image review}

Plaque contours were drawn delineating the ipsilateral, symptomatic, carotid plaque by experienced observers as described previously using dedicated vessel wall analysis software (VesselMASS, Leiden, the Netherlands) (2I). In case of doubt, a second highly experienced observer was consulted. There was a minimum period of two months between patient inclusion and MR contour drawing to assure that reviewers were blinded for clinical characteristics and other test results. Per slice, luminal contours were drawn on the 3D TOF MR images. Outer vessel wall contours were drawn using the pre-contrast TIw QIR TSE, post-contrast TIw 
QIR TSE, TIw TFE or T2w TSE MR images, in subsequent order. Luminal and outer vessel wall contours were transferred to the DCE-MRI images and, if necessary, contours were manually adjusted. The images acquired at each individual time frame were inspected and these images were shifted to correct for small patient displacements during the dynamic acquisition, if necessary. Outer plaque contours were corrected to include the adventitial vasa vasorum, which shows hyperenhancement after contrast material administration, while inner vessel wall contours were adjusted to avoid luminal partial volume effects. The adventitial region of the entire vessel wall region was automatically selected according to previously introduced criteria (22), i.e. all pixels within $0.625 \mathrm{~mm}$ of the outer wall contour in a region of the vessel wall with plaque (defined as having a wall thickness in excess of $1.5 \mathrm{~mm}$ ). Contour drawing and adjustment was performed blinded for clinical characteristics.

\section{Data analysis: DCE-MRI pharmacokinetic modeling}

Tissue CM concentrations were determined from the signal intensity time course as previously described (16). In brief, the CM concentrations were calculated using the Ernst equation, literature values for the longitudinal and transversal relaxation times for tissue (23) and the $r_{1}$ and $r_{2}$ relaxivity rates of the CM (24). Subsequently, $K^{\text {trans }}$ was determined with the Patlak model (25) using a population averaged phase-based arterial input function that was previously determined in the carotid artery (20). Resulting $\mathrm{K}^{\text {trans }}$ distribution in the vessel wall (i.e. region-of-interest between the inner and outer vessel wall) and adventitial region only was analyzed by calculating the $75^{\text {th }}$ percentile of the vessel wall and adventitial $K^{\text {trans }}$ distribution for the entire plaque region, respectively.

\section{Statistical analysis}

Univariate binary logistic regression was performed to select clinical risk factors with a relevant association (odds ratio $\geq 1.2$ ) with the clinically diagnosed stroke subtype. Multivariable logistic regression analysis was performed to study the independent association of plaque microvasculature characteristic with the clinically diagnosed type of event when adjusted for the selected clinical risk factors. The dependent variable in these models was the clinically diagnosed type of event ((ocular) TIA versus stroke), coded as 0 ((ocular) TIA) or I (stroke), respectively. All dichotomous risk factors were entered with the presence or absence of the parameter coded as I and 0, respectively. For logistic regression, the $95 \%$ confidence interval $(\mathrm{Cl})$ was determined. An odds ratio $\geq \mathrm{I}$ indicates a positive association of the clinical risk factors $/ \mathrm{K}^{\text {trans }}$ with a recent ischemic stroke compared to a recent (ocular) TIA. Statistical significance was set at $p \leq 0.05$. Since it was an explorative study, all statistical tests were two-tailed. Data are presented as mean \pm standard deviation or percentage, as appropriate. 


\section{RESULTS}

\section{Patient inclusion}

A total of 104 symptomatic patients with a recent (ocular) TIA or minor stroke were included. In 13 patients DCE-MRI was aborted due to ECG abnormalities. An extra 4 patients were excluded from final analysis due to low image quality leaving 87 patients $(70.1 \pm 8.4$ year, 56 male) for final analysis. Patient characteristics are shown in Table I. No significant difference in clinical risk factors between patient with (ocular) TIA or minor stroke was found.

\section{Association between event type versus clinical risk factors and $\underline{\mathbf{K}} \underline{\text { trans }}$}

Fifty-two patients (60\%) were diagnosed with an ocular TIA (7 patients) or TIA (45 patients), while 35 (40\%) were diagnosed with a stroke. Univariate analysis (Table 2) showed a non-significant positive association of diabetes mellitus, hypertension, and prior antiplatelet use and stroke subtype with $\mathrm{OR} \geq 1$ I.2. Multivariate analysis (Figure I) of the association between $\mathrm{K}^{\text {trans }}$ and the type of clinically diagnosed event adjusted for associated clinical risk factors $(O R \geq$ I.2) showed a significant association of adventitial $\mathrm{K}^{\text {trans }}(\mathrm{OR}=1.68, \mathrm{Cl}=1.06-2.68 ; \mathrm{p}=0.03)$ with the clinically diagnosed type of event (Table 3 ). No significant association was found between stroke subtype and vessel wall $\mathrm{K}^{\text {trans }}$ (Table 3).

\section{Table I: Patient characteristics}

Clinical characteristics for all patients and (ocular) / TIA versus stroke patients. a Data known for 86 out of 87 patients. bSeven patients experienced an ocular TIA.

\begin{tabular}{|c|c|c|c|}
\hline & (Ocular) TIA & Stroke & T-test \\
\hline Number of patients [n] & $52(60 \%)^{b}$ & $35(40 \%)$ & \\
\hline Age $[y]$ & $70.0 \pm 9.1$ & $70.1 \pm 7.3$ & $p=0.97$ \\
\hline Male sex (\%) & $33(55 \%)$ & $23(66 \%)$ & $p=0.83$ \\
\hline Body mass index $\left[\mathrm{kg} / \mathrm{m}^{2}\right]$ & $27.0 \pm 4.3$ & $26.4 \pm 3.6$ & $p=0.42$ \\
\hline Current smoking status (\%) & $10(17 \%)$ & $6(17 \%)$ & $p=0.85$ \\
\hline Diabetes mellitus (\%) & $7(12 \%)$ & $7(20 \%)$ & $p=0.42$ \\
\hline Hypertension (\%) & $27(45 \%)$ & $23(66 \%)$ & $p=0.20$ \\
\hline Hypercholesterolemia ${ }^{\mathrm{a}}$ (\%) & $23(38 \%)$ & $15(43 \%)$ & $p=0.99$ \\
\hline Prior statin use $(\%)$ & $24(40 \%)$ & 17 (49\%) & $p=0.89$ \\
\hline Prior platelet use $(\%)$ & $18(35 \%)$ & I 4 (40\%) & $p=0.66$ \\
\hline Time between event and MRI [d] & $41 \pm 19$ & $42 \pm 20$ & $p=0.86$ \\
\hline
\end{tabular}



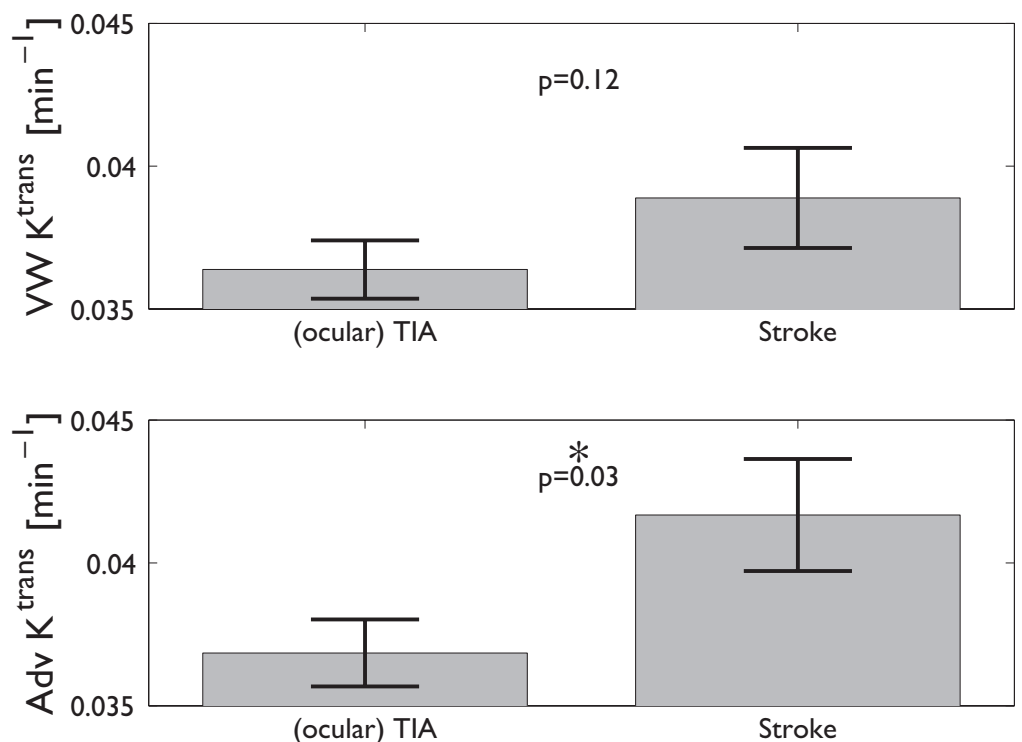

\section{Figure I}

Distribution of vessel wall (VW) and adventitial (Adv) $\mathrm{K}^{\text {trans }}$ based on the clinically diagnosed event (amaurosis fugax/ TIA versus stroke). $\mathrm{P}$-values from multivariate analysis between $\mathrm{K}^{\text {trans }}$ and type of clinically diagnosed event adjusted for associated clinical risk factors.

\section{Table 2: Univariate analysis}

Odds ratio $(95 \% \mathrm{Cl})$ and $\mathrm{p}$-value of univariate analysis of clinical risk factors with the type of recent clinically diagnosed event ((ocular) TIA versus stroke).

\begin{tabular}{ll}
\hline Clinical risk factor / Plaque characteristic & Odds Ratio $\mathbf{( 9 5 \% ~ C l ; ~} \mathbf{p}$-value $)$ \\
\hline Age, per 5 years & $0.99(0.76-1.29 ; p=0.92)$ \\
\hline Male sex & $1.10(0.45-2.7 I ; p=0.83)$ \\
\hline Body mass index, per I SD & $0.86(0.56-1.33 ; p=0.50)$ \\
\hline Current smoking status & $0.87(0.29-2.66 ; p=0.8 I)$ \\
\hline Diabetes mellitus & $1.61(0.5 I-5.07 ; p=0.42)$ \\
\hline Hypertension & $1.78(0.73-4.3 ; p=0.20)$ \\
\hline Hypercholesterolemia & $1.00(0.42-2.38 ; p=0.99)$ \\
\hline Prior statin use & $1.06(0.45-2.51 ; p=0.89)$ \\
\hline Prior antiplatelet use & $1.22(0.50-2.97 ; p=0.66)$ \\
\hline
\end{tabular}




\section{Table 3: Multivariate analysis correcting for associated clinical risk factors}

Odds ratio $(95 \% \mathrm{Cl})$ and corresponding $\mathrm{P}$-values from multivariate analysis of adventitial and vessel wall $\mathrm{K}^{\text {trans }}$ including associated clinical risk factors (odds ratio $\geq 1.2$ ) (shown in italics) with clinically diagnosed type of event ((ocular) TIA versus stroke, coded as 0 and I, respectively). ${ }^{*} \mathrm{p}<0.05$

\begin{tabular}{|c|c|}
\hline Plaque Characteristic & Odds ratio ( $95 \% \mathrm{Cl}$; p-value) \\
\hline $\begin{array}{l}\text { Vessel wall Ktrans, per I SD } \\
\text { Diabetes mellitus } \\
\text { Hypertension } \\
\text { Prior antiplatelet use }\end{array}$ & $\begin{array}{l}1.45(0.91-2.29 ; p=0.12) \\
I .52(0.46-5.04 ; p=0.49) \\
I .74(0.69-4.42 ; p=0.24) \\
0.82(0.48-1.4 I ; p=0.48)\end{array}$ \\
\hline $\begin{array}{l}\text { Adventitial Krans, per I SD } \\
\text { Diabetes mellitus } \\
\text { Hypertension } \\
\text { Prior antiplatelet use }\end{array}$ & $\begin{array}{l}\text { I. } .68(\text { I. } .06-2.68 ; p=0.03) * \\
\text { I.78 (0.53-6.0I; } p=0.35) \\
\text { I. } .65(0.64-4.23 ; p=0.30) \\
0.82(0.47-1.44 ; p=0.50)\end{array}$ \\
\hline
\end{tabular}




\section{Discussion}

In the present observational study in symptomatic patients with carotid plaque, a positive association of higher adventitial $\mathrm{K}^{\text {trans }}$ was found for patients who recently experienced a minor ischemic stroke compared to patients who were diagnosed with a recent transient ischemic attack (TIA) or ocular TIA, independent of associated clinical risk factors.

Previous studies have shown that stroke patients are at increased risk for a recurrent stroke compared to patients with an (ocular) TIA (8-10). The results from the present study suggest that differences between adventitial microvasculature within the symptomatic plaques of patients with (ocular) TIA and minor stroke exist between these two groups. Stroke patients demonstrated increased leaky plaque microvasculature compared to (ocular) TIA patients. The cross-sectional design of our study does not allow studying causality. Whether there is a causal relationship between differences in plaque type and the type of cerebrovascular event and whether $\mathrm{K}^{\text {trans }}$ can be used as an additional imaging marker to identify patients at risk needs to be studied in prospective serial studies. Additionally, the present study is an explorative study. Therefore, the results need to be confirmed by an independent study. Further, we cannot exclude that the results are influenced by the difficulty of diagnosing a TIA. Agreement on diagnosis among physicians is considerably lower for (ocular) TIA compared to stroke $(26,27)$. Therefore, the results of the current study may partly be explained by the heterogeneity among patients diagnosed with an (ocular) TIA.

\section{ConClusions}

The present observational study has shown a positive association of in leaky adventitial microvasculature of symptomatic carotid plaques in patients with a recent ischemic stroke compared to (ocular) TIA. Results suggest that differences of atherosclerotic plaques of patients with different risk profiles may exist, though confirmation of these results in future prospective studies is needed for more definitive conclusions. Further longitudinal research in larger cohorts is needed to investigate whether dynamic contrast-enhanced MRI model parameters of the plaque microvasculature may serve as additional imaging markers to predict (the type of) future cerebrovascular events. 


\section{REFERENCES}

I. Mackay J, Mensah G. The Atlas of Heart Disease and Stroke - Global Burden of Stroke. 2004.

2. Appelros P, Stegmayr B, Terent A. Sex Differences in Stroke Epidemiology: A Systematic Review. Stroke 2009;40:1082-1090.

3. Arboix A. Cardiovascular Risk Factors for Acute Stroke: Risk Profiles in the Different Subtypes of Ischemic Stroke. World journal of clinical cases 2015;3:4I8-429.

4. MacMahon S, Peto R, Cutler J, Collins R, Sorlie P, Neaton J et al. Blood Pressure, Stroke, and Coronary Heart Disease. Part I, Prolonged Differences in Blood Pressure: Prospective Observational Studies Corrected for the Regression Dilution Bias. Lancet 1990;335:765-774.

5. Jarrett RJ. Epidemiology and Public Health Aspects of Non-Insulin-Dependent Diabetes Mellitus. Epidemiol Rev 1989; |1:151-171.

6. Higa M, Davanipour Z. Smoking and Stroke. Neuroepidemiology |99|;|0:21 I-222.

7. Suk SH, Sacco RL, Boden-Albala B, Cheun JF, Pittman JG, Elkind MS et al. Abdominal Obesity and Risk of Ischemic Stroke: The Northern Manhattan Stroke Study. Stroke 2003;34:I586-I592.

8. Pendlebury ST, Rothwell PM. Risk of Recurrent Stroke, Other Vascular Events and Dementia after Transient Ischaemic Attack and Stroke. Cerebrovasc Dis 2009;27 Suppl 3: I- I I.

9. Stromberg S, Nordanstig A, Bentzel T, Osterberg K, Bergstrom GM. Risk of Early Recurrent Stroke in Symptomatic Carotid Stenosis. Eur J Vasc Endovasc Surg 2015;49:137-144.

10. Rothwell PM, Mehta Z, Howard SC, Gutnikov SA, Warlow CP. Treating Individuals 3: From Subgroups to Individuals: General Principles and the Example of Carotid Endarterectomy. Lancet 2005;365:256-265.

II. Fleiner M, Kummer M, Mirlacher M, Sauter G, Cathomas G, Krapf R et al. Arterial Neovascularization and Inflammation in Vulnerable Patients: Early and Late Signs of Symptomatic Atherosclerosis. Circulation 2004; I 10:2843-2850.

12. Koole D, Heyligers J, Moll FL, Pasterkamp G. Intraplaque Neovascularization and Hemorrhage: Markers for Cardiovascular Risk Stratification and Therapeutic Monitoring. Journal of Cardiovascular Medicine 20 I 2; 13:635$639610.2459 / J C M .2450 b 2013$ e3283590cd3283592.

13. Sluimer JC, Daemen MJ. Novel Concepts in Atherogenesis: Angiogenesis and Hypoxia in Atherosclerosis. J Pathol 2009;218:7-29.

14. Kerwin W. Quantitative Magnetic Resonance Imaging Analysis of Neovasculature Volume in Carotid Atherosclerotic Plaque. Circulation 2003; 107:85 I-856.

15. Kerwin WS, O'Brien KD, Ferguson MS, Polissar N, Hatsukami TS, Yuan C. Inflammation in Carotid Atherosclerotic Plaque: A Dynamic Contrast-Enhanced Mr Imaging StudyI. Radiology 2006;24I:459-468.

16. Gaens ME, Backes WH, Rozel S, Lipperts M, Sanders SN, Jaspers K et al. Dynamic Contrast-Enhanced Mr Imaging of Carotid Atherosclerotic Plaque: Model Selection, Reproducibility, and Validation. Radiology 2013;266:27I-279.

17. Kerwin WS, Oikawa M, Yuan C, Jarvik GP, Hatsukami TS. Mr Imaging of Adventitial Vasa Vasorum in Carotid Atherosclerosis. Magn Reson Med 2008;59:507-5I4.

18. Truijman MT, Kooi ME, van Dijk AC, de Rotte AA, van der Kolk AG, Liem Ml et al. Plaque at Risk (Parisk): Prospective Multicenter Study to Improve Diagnosis of High-Risk Carotid Plaques. International journal of stroke : official journal of the International Stroke Society 20I4;9:747-754.

19. Clinical Alert: Benefit of Carotid Endarterectomy for Patients with High-Grade Stenosis of the Internal Carotid Artery National Institute of Neurological Disorders and Stroke Stroke and Trauma Division. North American Symptomatic Carotid Endarterectomy Trial (Nascet) Investigators. Stroke 1991;22:816-817.

20. van Hoof RH, Hermeling E, Truijman MT, van Oostenbrugge RJ, Daemen JW, van der Geest RJ et al. Phase-Based Vascular Input Function: Improved Quantitative Dce-Mri of Atherosclerotic Plaques. Med Phys 20I5;42:46I9. 
21. Yuan C, Mitsumori LM, Ferguson MS, Polissar NL, Echelard D, Ortiz G et al. In Vivo Accuracy of Multispectral Magnetic Resonance Imaging for Identifying Lipid-Rich Necrotic Cores and Intraplaque Hemorrhage in Advanced Human Carotid Plaques. Circulation 200I; I04:205I-2056.

22. Sun J, Song Y, Chen H, Kerwin WS, Hippe DS, Dong L et al. Adventitial Perfusion and Intraplaque Hemorrhage: A Dynamic Contrast-Enhanced Mri Study in the Carotid Artery. Stroke 2013; 10.1 I 6 I / strokeaha. I I 000435.

23. Stanisz GJ, Odrobina EE, Pun J, Escaravage M, Graham SJ, Bronskill MJ et al. TI, T2 Relaxation and Magnetization Transfer in Tissue at 3t. Magn Reson Med 2005;54:507-5 12.

24. Pintaske J, Martirosian P, Graf H, Erb G, Lodemann KP, Claussen CD et al. Relaxivity of Gadopentetate Dimeglumine (Magnevist), Gadobutrol (Gadovist), and Gadobenate Dimeglumine (Multihance) in Human Blood Plasma at 0.2, I.5, and 3 Tesla. Invest Radiol 2006;41:213-22I.

25. Patlak CS, Blasberg RG, Fenstermacher JD. Graphical Evaluation of Blood-to-Brain Transfer Constants from Multiple-Time Uptake Data. J Cereb Blood Flow Metab 1983;3:1-7.

26. Castle J, Mlynash M, Lee K, Caulfield AF, Wolford C, Kemp S et al. Agreement Regarding Diagnosis of Transient Ischemic Attack Fairly Low among Stroke-Trained Neurologists. Stroke 20 I 0;4 I: I367- I370.

27. Leppala JM, Virtamo J, Heinonen OP. Validation of Stroke Diagnosis in the National Hospital Discharge Register and the Register of Causes of Death in Finland. Eur J Epidemiol 1999; I5:155-160. 



\section{Abstract}

\section{Objective}

To investigate the effect of a heart rate (HR) lowering agent (Ivabradine) on features of atherosclerotic plaque vulnerability with magnetic resonance imaging (MRI), ultrasound imaging, and histology.

\section{Approach and Results}

Atherosclerosis was induced in the abdominal aorta of 19 rabbits. Nine rabbits were treated with Ivabradine ( $17 \mathrm{mg} / \mathrm{kg} /$ day) during the entire study period. At week I4, imaging was performed. Plaque size was quantified on contrast-enhanced TI-weighted MR images. Microvascular flow, density, and permeability was studied with dynamic contrast-enhanced MRI. Plaque biomechanics was studied by measuring the aortic distension with ultrasound. After, animals were sacrificed and histology was performed.

HR was reduced by $16 \%(p=0.026)$ in Ivabradine-treated animals. No differences in absolute and relative vessel wall beat-to-beat distension were found, but due to the reduction in HR, the frequency of the biomechanical load on the plaque was reduced. Plaque size ( $M R$ and histology) was similar between groups. Although microvessel density (histology) was similar between groups, AUC and $\mathrm{K}^{\text {trans }}$, indicative for plaque microvasculature flow, density, and permeability, were decreased by $24 \%(p=0.029)$ and $32 \%(p=0.037)$, respectively. Macrophage content (relative RAMII positive area) was reduced by $44 \%(p<0.00 \mathrm{I})$ on histology in Ivabradine-treated animals.

\section{Conclusions}

$\mathrm{HR}$ lowering treatment with Ivabradine in an atherosclerotic rabbit model is associated with a reduction in vulnerable plaque features. The current study suggests that HR reduction may be beneficial for inducing or maintaining a more stable plaque phenotype. 


\section{INTRODUCTION}

\section{Increased heart rate and cardiovascular disease}

Cardiovascular diseases continue to be the major cause of morbidity and mortality in the worId. Large epidemiologic studies have shown that an elevated resting heart rate (HR) is an independent predictor of life expectancy, in subjects with and without diagnosed cardiovascular disease (I-3). Rupture of a vulnerable atherosclerotic plaque is an important underlying cause of cardiovascular events such as myocardial infarction and stroke. Experimental animal studies have demonstrated a link between HR and the burden of both coronary and carotid atherosclerosis (4-10). An increased HR may play a role in the progression of atherosclerosis due to the increased frequency of the biomechanical load imposed on the vessel wall (i.e. repetitive wall strain). Every heart beat, an atherosclerotic plaque is exposed to the arterial pressure wave, inducing wall stress, leading to repetitive plaque deformation (i.e. strain). Plaque strain may result in minor tissue damage, which can accumulate in time (crack propagation). This is also referred to as the fatigue hypothesis $(I|| 2$,$) . Hence, intensity of the biomechanical load$ on the plaque depends on heart rate as well as magnitude of repetitive wall stress. A high biomechanical load could lead to rupture of plaque microvessels, cap fissures, and ultimately, cap rupture. Ruptured microvessels or fissures may then provide a point of entry for inflammatory cells and erythrocytes (I3) into the plaque, leading to further plaque destabilization (I4).

\section{In vivo imaging of atherosclerosis and plaque strain}

Atherosclerosis can be visualized non-invasively using several imaging techniques. Ultrasound is frequently been used to assess the degree of stenosis of a blood vessel (15). In addition, ultrasound can be used to assess biomechanical properties of the vessel wall. The local wall strain coefficient is inversely related to the distensibility coefficient (16), i.e. the relative change in lumen diameter for a given pressure change. The distensibility coefficient in the carotid artery of humans is positively associated with the incidence of cardiovascular events (17). The (change in) lumen diameter can be measured over the cardiac cycle by phase tracking of raw radiofrequency ultrasound signals, providing high accuracy and precision (18). Magnetic Resonance Imaging (MRI) has emerged as a promising technique to visualize both plaque size and features of plaque vulnerability in vivo (19). Dynamic contrast-enhanced MRI (DCE-MRI) has developed into a tool for assessment of the atherosclerotic plaque microvasculature (20-30), which is thought to be a hallmark of plaque vulnerability (3I). It has been shown that both semi-quantitative (20) and quantitative (25-27) DCE-MRI parameters correlate with the amount of microvessels in the plaque. Previous research (22-24) has shown that anti-inflammatory treatment is associated with changes in DCE-MRI derived parameters.

Selective heart rate reduction Ivabradine (Servier, Suresnes, France) reduces the HR by specific and selective inhibition of the sinus node (32) with a high specificity (33), but does not affect cardiac contractility or blood pressure (BP) $(33,34)$. Thus, Ivabradine treatment provides a model to study the effects of $\mathrm{HR}$ reduction on plaque size and vulnerability. A pre-clinical histopathological study has shown that Ivabradine treatment reduced atherosclerosis in ApoE1- mice (35). However, no in vivo imaging was performed. In addition dynamic processes, like (leaky) plaque microvasculature and strain were not investigated. 


\section{Aim of the study}

In the present study, the effect of a reduced HR by Ivabradine treatment on atherosclerotic rabbit plaques was studied. We aimed to examine plaque burden and (leaky) plaque microvasculature with MRI. Local absolute and relative distension of the vessel wall was assessed using high-frame rate ultrasound. Features of plaque vulnerability (i.e. relative lipidrich necrotic core size, microvessel density, and macrophage content) were quantified using histology. 


\section{Material ANd Methods}

\section{Experimental methods}

Animal Model

All experiments were approved by the Maastricht University Animal Experiments Committee. Atherosclerosis was induced in nineteen 12 weeks old male New Zealand white rabbits (Charles River, Romans, France) using a modified version of a previous protocol (36). The experimental design is presented in figure I. In short, animals were given a I.0\% cholesterolenriched diet for 10 weeks, followed by a $0.3 \%$ cholesterol-enriched diet for 4 weeks. During the study, animals were given normal drinking water $(n=10)$ or drinking water with Ivabradine $(n=9)(17 \mathrm{mg} / \mathrm{kg} /$ day $)$. The dosage was adjusted according to animal weight and water consumption on a weekly basis.

After two weeks, animals underwent a balloon denudation of the abdominal aorta. During this procedure, rabbits were anesthetized using ketamine $(15 \mathrm{mg} / \mathrm{kg})$ and medetomidine $(0.25 \mathrm{mg} / \mathrm{kg})$ and intubated with an endotracheal tube. Active oxygen ventilation with I- $2 \%$ isoflurane inhalation gas was performed during the rest of the experiment. The left carotid artery was exposed, and a 4-F catheter introducer sheath was inserted (Avanti Plus; Cordis Europe, Brussels, Belgium) until the aortic arch was reached. Next, a guidewire (Radiofocus Guidewire M; Terumo Europe, Brussels, Belgium) was inserted through the sheath until in the descending aorta was reached. A 3-F Fogarty embolectomy catheter (Edwards Lifesciences, Irvine, California) was then inserted, and was positioned $1 \mathrm{~cm}$ proximally to the aortic bifurcation. From this point, the catheter was inflated and withdrawn over $5 \mathrm{~cm}$ for three times. After removal of the devices, the carotid artery was ligated and the wound was sutured. Animals were hourly monitored during post-surgery recovery by monitoring heart rate, oxygen saturation, and breathing frequency.

After 14 weeks, animals underwent US and MR imaging of the abdominal aorta under general anesthesia as described before. Before anesthesia, conscious heart rate was measured using pulse oximetry. During ultrasound imaging, systolic and diastolic BP were measured in the auricular artery. After the imaging experiments, animals were sacrificed by an overdose sodium pentobarbital.

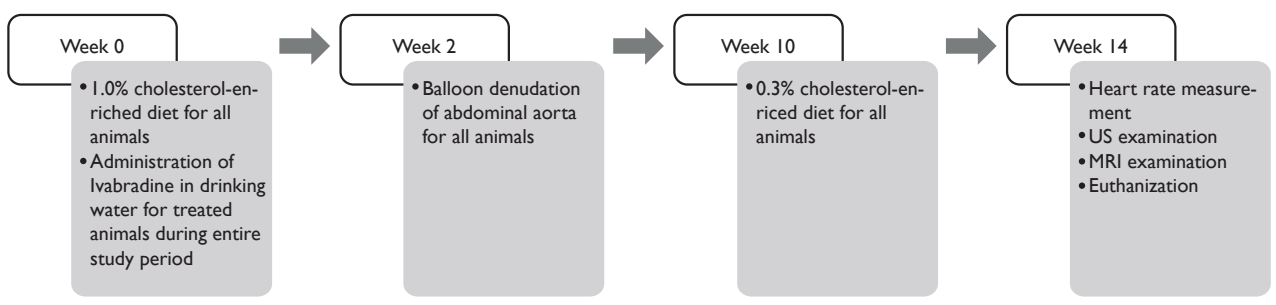

\section{Figure I}

The experimental design. Two weeks after initiation of $1.0 \%$ cholesterol-enriched diet, the animals receive a balloon injury. At week 10, they are switched from I.0\% to $0.3 \%$ cholesterol-enriched diet. Fourteen weeks after diet initiation the animals undergo measurement of the heart rate and US and MR imaging before euthanisation. 


\section{US Protocol}

The distension, i.e. the maximal change in aortic diameter over the cardiac cycle, was assessed using a PICUS ultrasound scanner (Esaote Europe, Maastricht, Netherlands) with a $7.5 \mathrm{MHz}$ linear array probe. The system was operating in multiple $\mathrm{M}$-mode, producing $28 \mathrm{M}$-mode lines at a frame rate of $380 \mathrm{~Hz}$ (18), covering a segment of the abdominal aorta of $26.5 \mathrm{~mm}$. The radiofrequency-data were sampled at $33.3 \mathrm{MHz}$.

\section{MRI Protocol}

MRI experiments were performed on a 7T Small Animal Scanner (Biospec 70/30, Bruker, Ettlingen, Germany). The animal was positioned in the magnetic field and, after initial scout acquisitions, the aortic bifurcation was identified. A TIw two-dimensional double inversion-recovery (DIR) black blood (BB) RARE sequence (37) (TR/TE/TI: 1000/10/350 ms, RARE factor 2 , field of view (FOV) $12 \times 12 \mathrm{~cm}$, acquired/reconstructed matrix size $384 \times 384 / 5|2 \times 5| 2$, 20 transversal adjoining slices with a thickness of $3 \mathrm{~mm}$ ) was acquired with the bottom slice positioned at the aortic bifurcation. After visual inspection of these images, a single slice with the highest plaque burden was selected. DCE-MRI was performed at this location using a two-dimensional inversion-recovery black blood turbo spin echo sequence (20) (TR/TE/TI=300/9.5/120 ms, field of view $12 \times 12 \mathrm{~cm}$, acquired/reconstructed matrix size $192 \times 192 / 384 \times 384$ with a thickness of $3 \mathrm{~mm}$ ) with an acquisition time of 7.2 seconds per time frame. After one minute, $0.2 \mathrm{mmol} / \mathrm{kg}$ body weight Gadobutrol (Gadovist, Bayer HealtCare, Berlin, Germany) was injected intravenously through a marginal ear vein manually at a rate of $0.5 \mathrm{ml} / \mathrm{sec}$, followed by a $5 \mathrm{ml}$ saline chaser at the same rate. Imaging was continued for 120 time frames. After, contrast-enhanced TIw DIR BB imaging was performed again using the same pulse sequence parameters as listed above.

\section{Ultrasound image review and data analysis}

The anterior and posterior lumen-wall interface were identified manually for each M-line. A wall track system $(38,39)$ was used to obtain the diameter waveforms over the cardiac cycle. A temporal correlation window (26 ms, 10 sample points) was used for assessment of diameter waveforms with high temporal resolution. Absolute distension of the lumen was calculated as the difference between systolic maximal diameter and preceding diastolic minimal diameter, while relative distension was determined as the absolute distension divided by the diastolic minimal diameter.

\section{MR image review and data analysis}

Lumen and outer vessel wall contours were drawn using dedicated vessel wall analysis software (VesselMASS, Leiden University Medical Center, Leiden, The Netherlands). Mean plaque burden was quantified on contrast-enhanced TIw BB MR images. From these, contours were transferred to the DCE MR images. Inner contours were adjusted to prevent partial volume effects at the plaque-lumen border. The DCE-MRI signal intensity time curves (relative to baseline values before contrast injection) of the atherosclerotic vessel wall were quantified semi-quantitatively (area-under-the-curve, AUC) and quantitatively ( $\mathrm{K}^{\text {trans }}$, indicative for plaque microvasculature flow, density, and permeability) with pharmacokinetic modeling using 
a reference region model (40). With this method, the arterial input function is not required, allowing quantitative analysis of BB DCE-MRI acquisitions. The AUC was calculated by numeric integration. In line with previous research (20), integration is performed from 0- 7 minutes after contrast injection. For the quantitative analysis, literature values for the blood perfusion-vessel permeability product $\left(K^{\text {trans }}\right)$ and the extravascular-extracellular volume fraction $\left(v_{e}\right)$ (4I) for the reference region (skeletal muscle) were used.

\section{Histological and immunohistochemistry analysis}

After euthanasia, the abdominal aorta was dissected from the aortic bifurcation to $\sim 7$ centimeters proximally. Specimens were fixated using I\% paraformaldehyde for 24 hours, cut into 5 -mm-thick tissue slices, processed and embedded in paraffin and sectioned (4- $\mu \mathrm{m}$ sections). An Elastica von Gieson (EvG) staining was used to visualize the inner and outer elastic laminae and the lipid core. Presence of cap fissures was examined using a haematoxylin eosin stain. Immunohistological staining for rabbit macrophages and microvessels was performed using a primary antibody against monocytes/macrophages (clone RAMI I; Dako North America, Carpinteria, California) and a primary antibody against CD3I (clone JC70A, Dako), respectively. RAMII and CD3 I slides were counterstained with hematoxylin. Matching of histologic sections with MRI slices was performed based on their longitudinal position relative to the aortic bifurcation, taking into account an ex vivo shrinking of approximately I5\%. Digital images from slides matched to the DCE-MRI slice were analyzed with morphometric analysis software. EvG and RAMII images were analyzed using QWin V3 (Leica, Cambridge, Great Britain) and CD3I images using Ventana Image Viewer (Ventana, Tucson, Arizona). Median intima size and relative lipid rich necrotic core size (relative to the intima size) were determined on the EvG stained sections. Lipid rich necrotic core was identified by the area of necrotic material in which residual cholesterol crystals were seen. The relative macrophage content was determined as the amount of RAMII positive area related to the total plaque area. Presence of macrophages in the proximity (approximately $50 \mu \mathrm{m}$ ) of the lumen was scored semi-quantitatively on a three point scale ranging from no luminal macrophages (0) to many luminal macrophages (2). Microvessel density was determined as the number of microvessels per $\mathrm{mm}^{2}$ for the entire vessel wall. All histological analyses were performed blinded to the other study results and animal characteristics.

\section{Statistical Analysis}

The differences between study parameters of the two groups were studied using an independent Mann-Whitney $\cup$ Test. Study parameters were divided into cardiovascular parameters (conscious and anesthetized heart rate, and systolic and diastolic BP), plaque burden parameters (mean vessel wall area on MR images and mean histological intimal area), plaque biomechanical parameters (absolute and relative vessel wall distension), and plaque vulnerability markers (plaque enhancement from DCE-MRI determined using AUC and $\mathrm{K}^{\text {trans }}$, and relative lipid-rich necrotic core size, microvessel density, and macrophage content from histology). Relationships between macrophage content and DCE-MRI parameters (AUC and $\mathrm{K}^{\text {trans }}$ ), and between microvessel density, AUC, and $\mathrm{K}^{\text {trans }}$ were assessed using a Spearman's correlation coefficient. All results were considered significant for a $p$-value $<0.05$. 


\section{RESULTS}

The number of animals in which successful experiments were performed is listed in Table I. During the study, two rabbits (one non-treated and one Ivabradine-treated) died due to complications during the balloon denudation surgery. One Ivabradine-treated animal died during the study period due to icterus, caused by the cholesterol-enriched diet (diagnosed by a veterinary doctor at autopsy). For one non-treated animal, contrast injection failed. Therefore, this animal was excluded from MRI and histological analysis. For one Ivabradine-treated animal, BP measurements failed.

\section{Table I}

Number of successfully performed procedures in the present study.

\begin{tabular}{lll}
\hline Experiment & Non-treated & Ivabradine-treated \\
\hline Included animals & 10 & 9 \\
Conscious HR & 9 & 7 \\
Blood Pressure & 9 & 6 \\
Ultrasound Imaging & 9 & 7 \\
Magnetic Resonance Imaging & 8 & 7 \\
Histology & 8 & 7 \\
\hline
\end{tabular}

\section{Heart rate and blood pressure}

As shown in Table 2, HR for Ivabradine-treated animals was significantly lower than for non-treated animals, both under conscious (Figure 2A) conditions (mean reduction 16\%, $p=0.026$ ), and under anesthesia (mean reduction $15 \%, p=0.031$ ). No significant differences were found for the systolic and diastolic BP measured under anesthesia between groups.

\section{Plaque biomechanics}

No differences were found in beat-to-beat plaque biomechanical parameters between the two groups (Table 2). However, due to the HR reduction, the frequency that the aorta is exposed to the pulsatile BP is decreased for Ivabradine-treated animals.

\section{Plaque features}

Examples of MR images and histological sections (HE and RAMI I antibody against macrophages) for non-treated and Ivabradine-treated animals are shown in Figure 3 and 4, respectively. No significant differences were found for plaque burden related parameters (Table 2), as determined using MRI (mean entire vessel wall area) and histology (mean intima area). Also, no differences were found for the relative lipid core size between the two groups. The relative RAMI I positive area (macrophages) was decreased from $30.6 \pm 4.3 \%$ for non-treated animals to $17.2 \pm 7.6 \%$ for Ivabradine-treated animals $(p<0.00 \mathrm{I})$. Plaques of the Ivabradine-treated group showed less macrophages in proximity of the vessel lumen as compared to non-treated animals (Figure 5). Presence of cap fissures was not observed in the specimens. Analysis of the DCE-MRI data (Figure 6) revealed a reduction in vessel wall enhancement, both semi-quantitatively $(24 \%$ reduction in the $A \cup C$ after seven minutes, $p=0.029)$ and quantitatively $(32 \%$ re- 
duction for $K^{\text {trans }}, p=0.037$ ) for rabbits treated with Ivabradine, indicative for a decrease in plaque microvasculature flow, density, and/or leakiness. A strong positive correlation between AUC and Krans (Spearman's $\rho=0.70, p=0.004$ ) was observed. No difference in microvessel

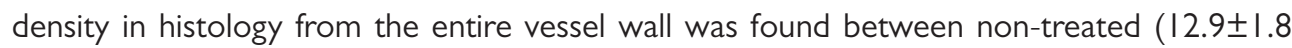
$\mathrm{mm}-2)$ and lvabradine-treated $(9.8 \pm 1.8 \mathrm{~mm}-2)$ animals $(p=0.397)$. Microvessel density in histology correlated with AUC (Spearman's $\rho=0.53, p=0.04 \mathrm{I}$ ), but not with K ${ }^{\text {trans }}$ (Spearman's $\rho=0.17, p=0.550)$. A modest, non-significant, correlation between the relative RAMI I area and the signal enhancement on DCE-MRI with a Spearman's $\rho$ of $0.46(p=0.08 \mathrm{I})$ for the AUC after 7 minutes and a Spearman's $\rho$ of $0.49(p=0.062)$ for $K^{\text {trans }}$ was found.

\section{Table 2}

Study parameters (mean \pm standard error) for the present study. Parameters are reported as mean values \pm standard error. Number of successful experiments that were performed is indicated $\left({ }^{1} n=9,{ }^{2} n=8,{ }^{3} n=7,{ }^{4} n=6\right)$. AUC 7 min and $\mathrm{K}^{\text {trans }}$ represent measurements of the plaque microvasculature, RAMII is a measurement of the plaque macrophage content. Used abbreviations: heart rate (HR); blood pressure (BP); Elastica von Gieson (EvG); area-under-the-curve (AUC).

\begin{tabular}{|c|c|c|c|c|}
\hline Category & Parameter & Non-treated & Ivabradine-treated & P-value \\
\hline \multirow[t]{4}{*}{ Cardiovascular } & Conscious HR [bpm] & $186 \pm 7.0^{1}$ & $157 \pm 9.3^{3}$ & 0.03 \\
\hline & Anesthesia HR [bpm] & $164 \pm 5.9^{1}$ & $140 \pm 6.4^{3}$ & 0.03 \\
\hline & Systolic BP [mmHg] & $63 \pm 3.4^{1}$ & $71 \pm 7.9^{4}$ & 0.27 \\
\hline & Diastolic BP [mmHg] & $45 \pm 3.4^{\prime}$ & $53 \pm 6.8^{4}$ & 0.33 \\
\hline \multirow[t]{2}{*}{ Plaque burden } & MR plaque size $\left[\mathrm{mm}^{2}\right]$ & $11.0 \pm 0.9^{2}$ & $10.8 \pm 1.1^{3}$ & 0.54 \\
\hline & EvG intima size $\left[\mathrm{mm}^{2}\right]$ & $6.80 \pm 1.03^{2}$ & $6.61 \pm 1.19^{3}$ & 0.91 \\
\hline \multirow[t]{2}{*}{ Biomechanics } & Absolute distension [mm] & $0.066 \pm 0.017$ & $0.064 \pm 0.018^{3}$ & 0.84 \\
\hline & Relative distension [\%] & $1.9 \pm 0.4^{2}$ & $1.9 \pm 0.3^{3}$ & 0.84 \\
\hline \multirow[t]{5}{*}{ Plaque vulnerability } & AUC 7min [a.u.] & $791 \pm 60^{2}$ & $604 \pm 44^{3}$ & 0.03 \\
\hline & $\mathrm{K}^{\text {trans }}\left[\mathrm{min}^{-1}\right]$ & $0.25 \pm 0.02^{2}$ & $0.17 \pm 0.02^{3}$ & 0.04 \\
\hline & Microvessel density $\left[\mathrm{mm}^{-2}\right]$ & $12.9 \pm 1.8^{2}$ & $9.8 \pm 1.8^{3}$ & 0.40 \\
\hline & Relative lipid core size [\%] & $1.7 \pm 0.4^{2}$ & $1.5 \pm 0.3^{3}$ & 0.66 \\
\hline & Relative RAMII [\%] & $30.6 \pm 1.5^{2}$ & $17.2 \pm 2.9^{3}$ & $<0.01$ \\
\hline
\end{tabular}




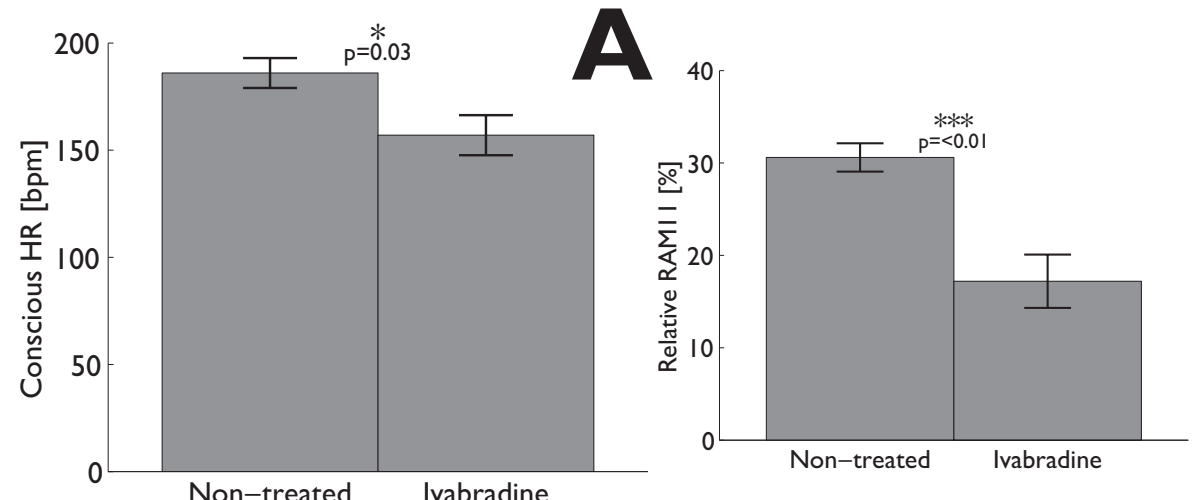

B
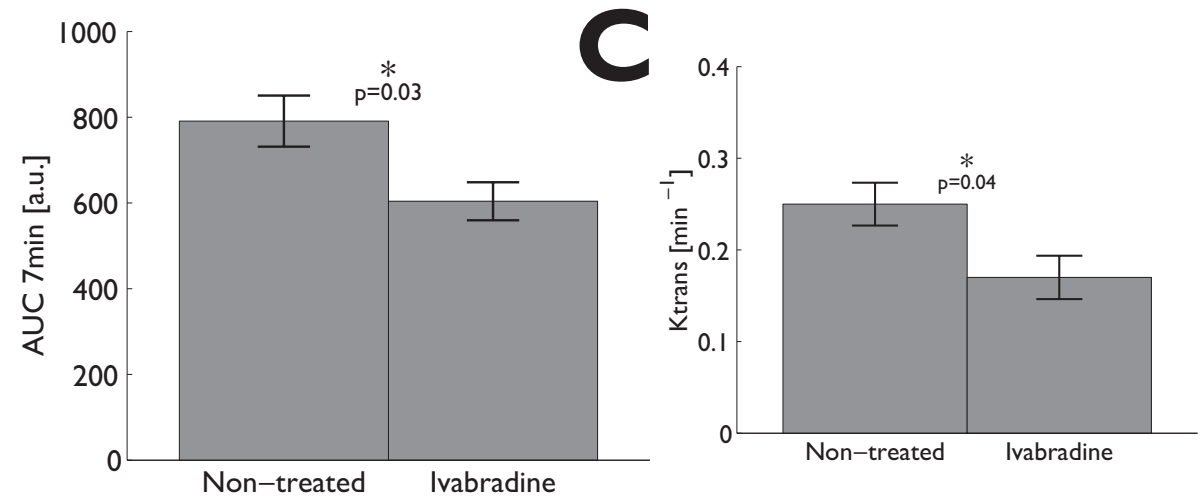

\section{Figure 2}

Bar graphs showing the differences between non-treated and Ivabradine-treated animals (mean \pm standard error). Panel A shows the conscious heart rate (HR); panel B relative RAMII positive area in histological sections; panel C and $\mathrm{D}$ show the area-under-the-curve (AUC) and $\mathrm{K}^{\text {trans }}$ determined from analysis of DCE-MR images.
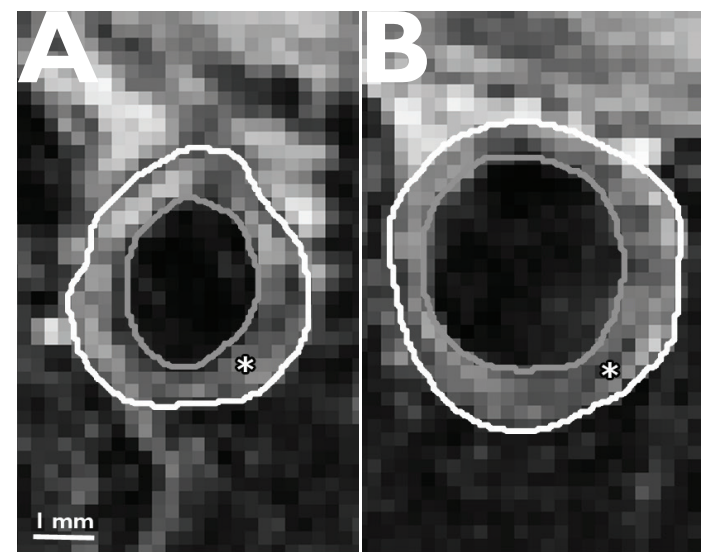

\section{Figure 3}

Contrast-enhanced TIw BB MR image of atherosclerotic plaque for a non-treated rabbit (panel A) and lvabradine treated rabbit (panel B). In the panels, the outer vessel wall is indicated by the white line and the lumen by the gray line. The atherosclerotic plaque is indicated by the asterisk $(*)$. 

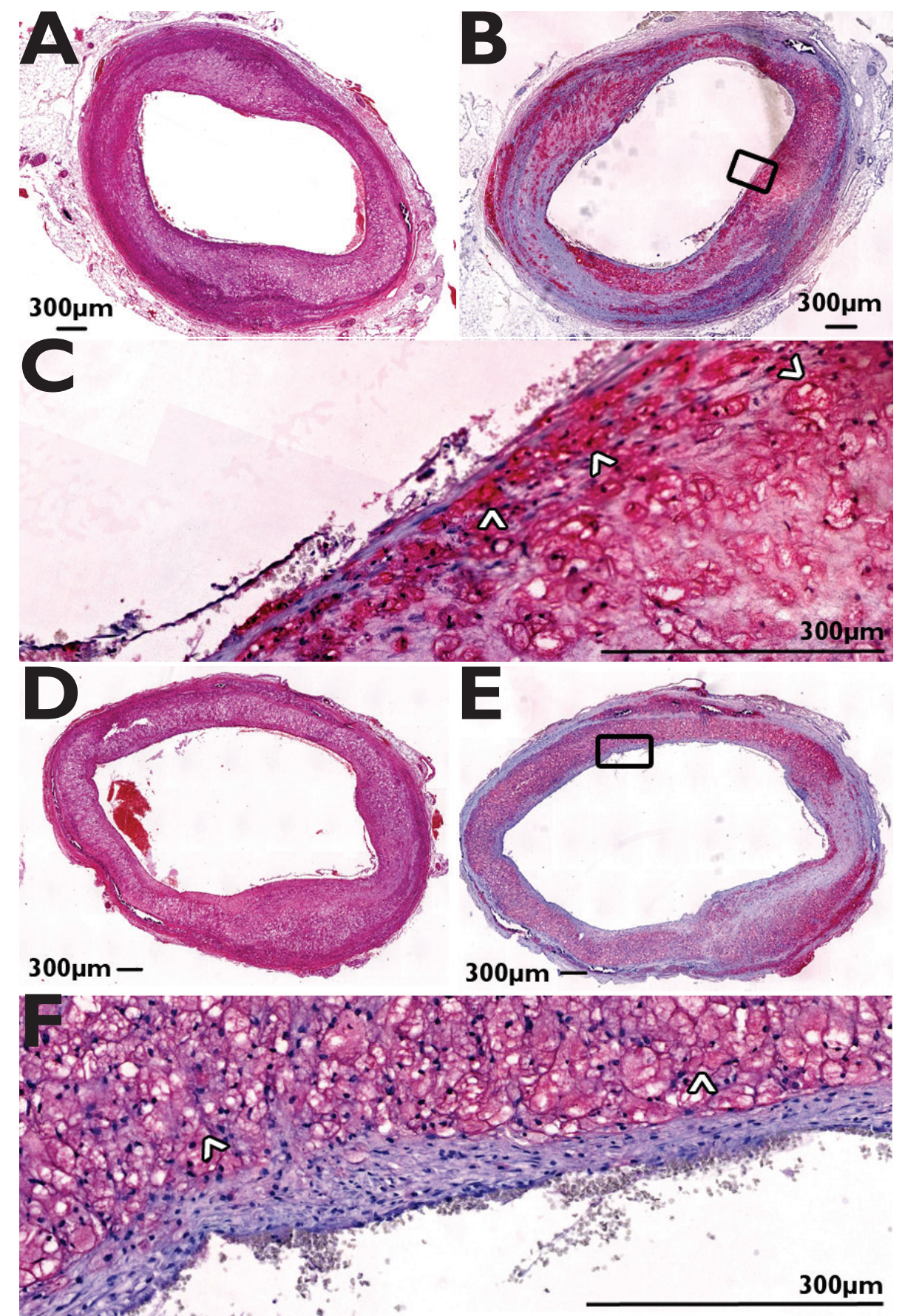

\section{Figure 4}

Histological sections of atherosclerotic plaque for a non-treated (panels $A-C$ ) and Ivabradine treated (panels D-F) rabbit. Panel $A$ and $D$ show HE sections and panel $B$ and $E$ a slide stained for macrophages using RAMII antibody (red), with magnifications in panel $\mathrm{C}$ and $\mathrm{F}$. 

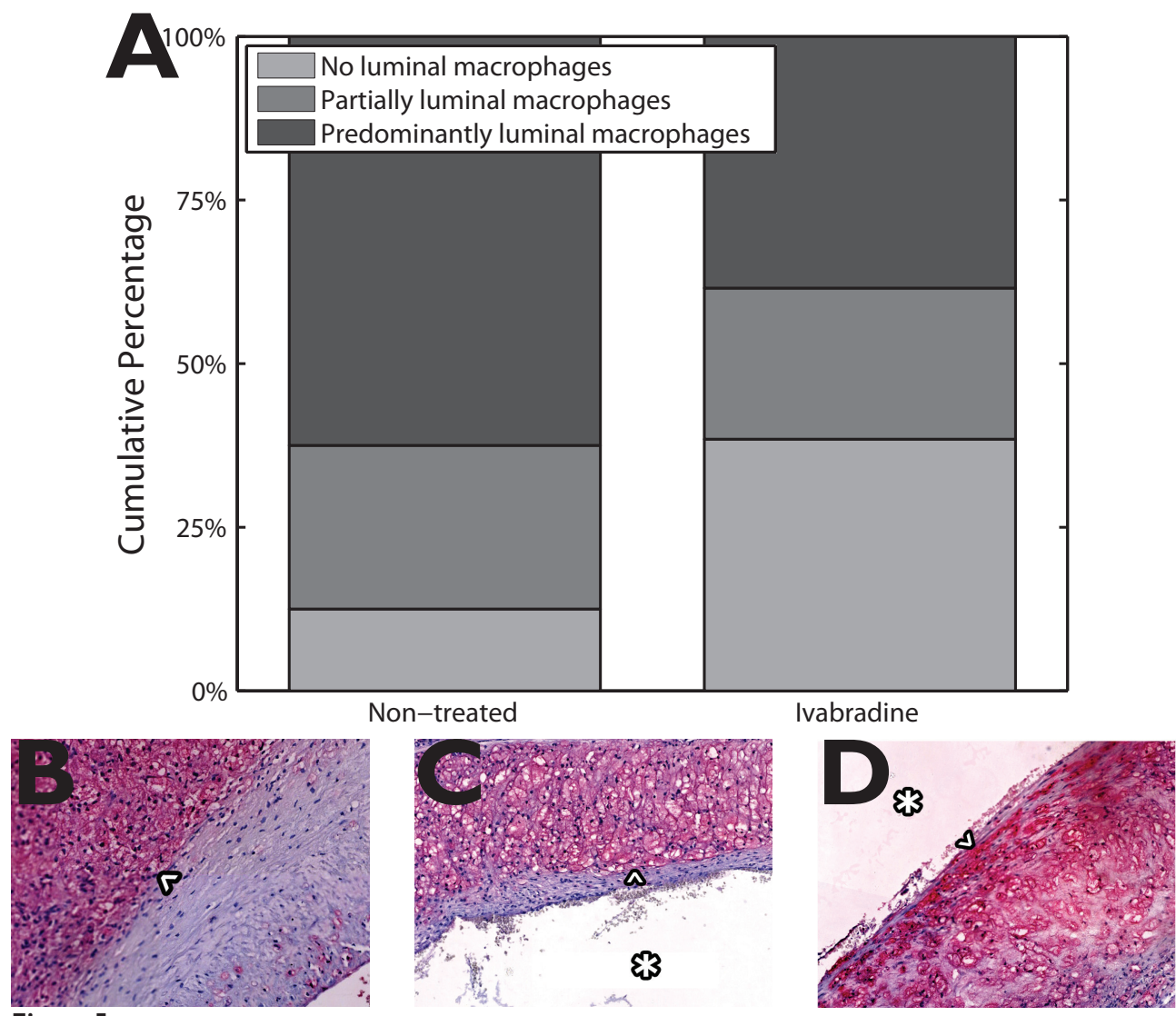

\section{Figure 5}

Presence of macrophages in the proximity of the vascular lumen using a semi-quantitative three point scale for nontreated (eight animals, two sections per animal) and Ivabradine-treated animals (seven animals, 2 sections per animal). Histological sections are divided into three categories ranging from 0 (no luminal macrophages; example image shown in panel B) via I (partially luminal macrophages; example in panel C) to 2 (predominantly luminal macrophages; example in panel D). The displayed scale bar is applicable for all histological images and the luminal side is indicated by an asterisk $\left(^{*}\right)$ in all histological images. 


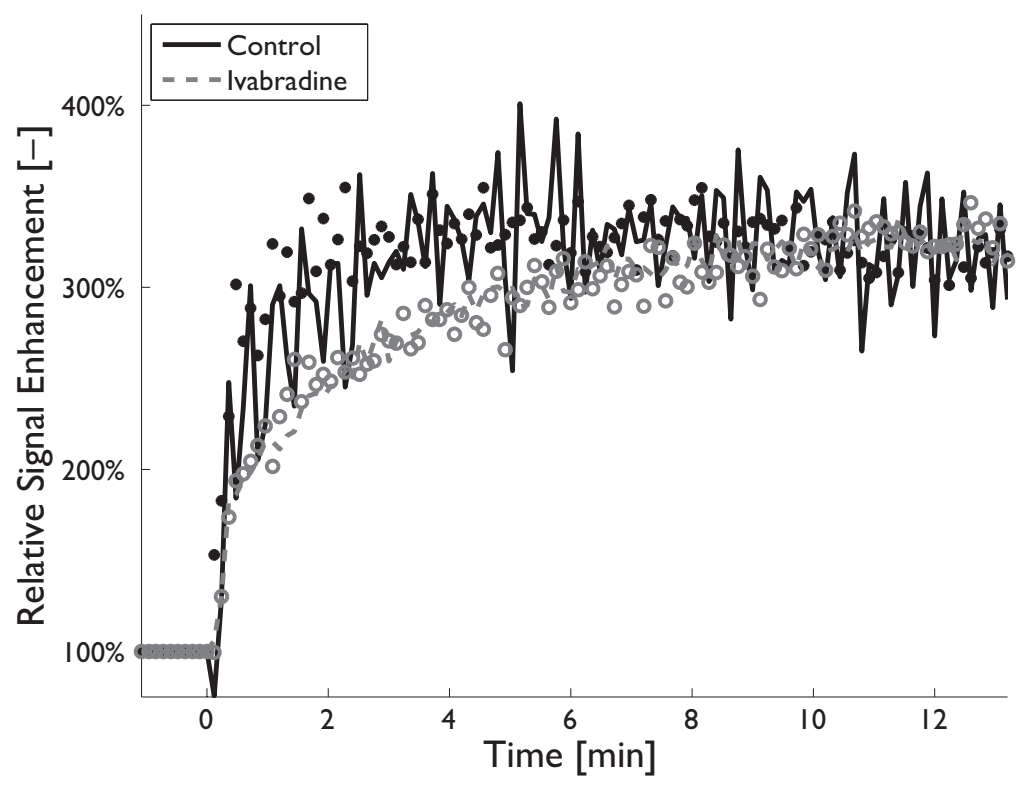

\section{Figure 6}

Dynamic contrast-enhanced MRI signal-intensity-time curves for a non-treated (black filled circles) and Ivabradinetreated animal (red open circles) showing the relative signal enhancement in the atherosclerotic vessel wall after contrast injection. Pharmacokinetic modelling curves are shown as black solid lines (non-treated animals) and gray dotted lines (Ivabradine-treated animals). Enhancement curves for Ivabradine-treated animals show a delayed enhancement compared to non-treated animals, which is indicative for a decrease in plaque microvasculature. 


\section{Discussion}

The major novelty of the present study is the use of sophisticated noninvasive imaging techniques in combination with histology to study the effect of the HR lowering agent Ivabradine on atherosclerotic plaques. This allowed us to demonstrate that HR lowering treatment was associated with a reduction in macrophage content and changes in contrast enhancement of rabbit plaques, indicative for less inflammation and a decrease in plaque microvasculature flow or leakiness, respectively. In contrast, no differences in plaque burden, microvessel density, and vessel wall distension were observed between treated and non-treated rabbits.

DCE-MRI enables to study not only plaque microvascular density, but also microvascular leakiness. Our qualitative as well as quantitative DCE-MRI parameters, indicative for microvascular density, flow, and leakiness, showed a reduction after Ivabradine treatment, while the microvessel density on histology showed no difference between non-treated and Ivabradine-treated animals. Together, these results suggest a reduction of the plaque microvasculature leakiness. With a decreased $\mathrm{HR}$, the vascular wall has a lower exposure to oscillatory tensile and wall shear stresses $(42,43)$, decreasing the frequency of the biomechanical load. A reduction in shear and tensile stress is thought to reduce endothelial damage, which leads to a decreased endothelial permeability to inflammatory mediators $(42,43)$, in line with a decrease in microvascular leakiness and a reduction in macrophage content in the treatment group. Alternatively, the treatment may have reduced microvessel rupture, since it was previously suggested that pressure gradients within the plaque that occur during every heart beat lead to microvessel rupture (44). In line with a decrease in endothelial permeability, Ivabradine-treated animals showed less luminal macrophages (Figure 3), while in both animal groups no cap fissures were found.

In the present study, HR reduction was induced during the entire study period. Future studies with a longitudinal design in which $\mathrm{HR}$ reduction is performed after atherosclerosis induction in animal models are warranted to investigate whether HR reduction reduces plaque vulnerability or mitigates the formation of plaque vulnerability. Future studies should also investigate the effect of Ivabradine and more commonly applied HR reducing agents, i. e. beta blockers, on plaque vulnerability in patient studies.

For the current study, a non-significant, albeit modest, correlation between RAMI I positive area (relative macrophage content) and DCE-MRI parameters was found. These results are in line with previous studies $(20,23,28-30)$, where a weak to modest correlation between plaque inflammation and microvasculature was reported. The present study supports the concept that there is a complex interplay between macrophages and plaque microvasculature (28). Macrophages have a high metabolic rate, which can lead to plaque hypoxia, which is known to stimulate angiogenesis (45). The newly formed, potentially immature angiogenic microvessels may then allow additional inflammatory cells to enter the plaque tissue. The extent of

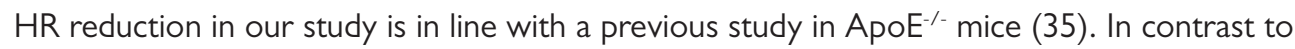
this previous study, in our rabbit model administration of Ivabradine did not lead to smaller plaques on MRI and histology in the treatment group. The differences in plaque burden between our study and previous findings might be related to differences between the two animal 
models (46-48). In the previous study (35), administration of Ivabradine did lead to reduced endothelial dysfunction and exerted potent anti-oxidative effects in $\mathrm{ApoE}^{-/-}$mice. Following these results, further in vitro investigations were performed to investigate if Ivabradine exerted direct effects on the endothelium. These investigations revealed no differences of the vasodilatation of vessel segments or protein expression of cultured bovine aortic endothelial cells, suggesting that direct anti-oxidative effects of Ivabradine are not involved in the reduction of endothelial dysfunction. Microvessel density in the non-treated group was higher than our findings in a previous study (36). The higher microvessel density may be related to the extended cholesterol-enriched diet period of the present study.

A number of mechanisms of Ivabradine next to a reduction of the HR were observed that improve myocardial structure and function, including anti-inflammatory effects, myocardial infarct size reduction, a reduction of the formation of reactive oxygen species, increased coronary flow reserve, and an increased perfusion of the coronaries (49). While previous research (50) did show improvements in clinical outcome (cardiovascular death or hospital admission for worsening of heart failure) in patients with heart failure after HR reduction with Ivabradine, a recent study in patients with stable coronary disease without clinical heart failure (5I), showed that additional treatment with Ivabradine did not lead to a reduction in cardiovascular death or nonfatal myocardial infarctions. Therefore, it is of great interest to further investigate which patient groups may and may not benefit from HR lowering treatment with Ivabradine $(52,53)$. In the present study, the animals' HR was measured once before the MR and US examinations performed after 14 weeks. Continuous monitoring of the HR during the study period using telemetric devices was not performed due to logistical reasons. Compared to previous research (20-24) within the present study, analysis of the DCE-MRI data was extended with quantitative analysis using a reference region method. The reference region method does not require a vascular input function and thus allows quantitative analysis of black blood (BB) DCE-MRI data. It is able to account for inter-subject variations due to contrast bolus differences. Use of BB images allows for more reliable determination of the plaque microvasculature near the lumen, which is important in the small-sized aortic rabbit plaques. Our study shows a strong, significant correlation between the semi-quantitative AUC and quantitative $\mathrm{K}^{\text {trans }}$ parameter.

Our study has a number of limitations. First, we have chosen Ivabradine as a heart-rate reducing agent to investigate the specific effect of heart-rate reduction, while not affecting cardiac contractility or blood pressure (BP) $(33,34)$. However, a recent study on mouse ventricular myocytes has shown that Ivabradine blocks the IhERGI potassium current, which appears to contribute to a prolonged cardiac repolarization (54). Therefore, we cannot exclude that the present findings have been influenced by other actions of Ivabradine. Second, DCE-MR imaging was performed on a single axial slice. Previous research (20-24) has shown that this technique is able to quantify the plaque microvasculature and measure the effect of therapeutic interventions. Recently, a 3D acquisition techniques to quantify plaque microvasculature in rabbits have been introduced (55). This 3D technique provides increased spatial coverage as compared to the 2D technique, although at the expense of temporal resolution.Finally, from 
previous studies it is known that the cardiovascular hemodynamics are affected by the administration of general anesthesia, with the effect differing between species and strains and the type of anesthetics and administered dose (56-58). Isoflurane is known to have vasodilative properties, which may lead to hypotension (58). General anesthesia by administration of a combination of ketamine and xylazine should potentially not lead to hypotension and may be more suited to perform measurements under physiological conditions that resemble normal conditions more closely. However, the increased wake-up and recovery period may increase complications (58).

\section{CONCLUSION}

Our study suggests that a lower HR may lead to reduced plaque microvessel leakiness and reduced plaque inflammation in a model of atherosclerotic rabbits. Increased plaque inflammation and plaque microvasculature leakiness have been suggested as key features of plaque vulnerability. The present study suggests that exposure of the atherosclerotic vessel wall to biomechanical load at a higher repetition frequency contributes to plaque vulnerability. HR reduction may be a potential target for plaque stabilization. Whether these preclinical findings can be translated into clinical practice remains to be determined. 


\section{REFERENCES}

I. Fox K, Borer JS, Camm AJ, Danchin N, Ferrari R, Lopez Sendon JL et al. Resting Heart Rate in Cardiovascular Disease. J Am Coll Cardiol 2007;50:823-830.

2. Lévy S, Guize L. [Heart Rate, a Major Prognostic Factor of Cardiovascular Risk]. Therapie 2006;6I:I I 5-II 9.

3. Palatini P. Heart Rate as an Independent Risk Factor for Cardiovascular Disease: Current Evidence and Basic Mechanisms. Drugs 2007;67 Suppl 2:3-13.

4. Bassiouny HS, Zarins CK, Lee DC, Skelly CL, Fortunato JE, Glagov S. Diurnal Heart Rate Reactivity: A Predictor of Severity of Experimental Coronary and Carotid Atherosclerosis. Journal of cardiovascular risk 2002;9:331338.

5. Kaplan JR, Manuck SB, Adams MR, Weingand KW, Clarkson TB. Inhibition of Coronary Atherosclerosis by Propranolol in Behaviorally Predisposed Monkeys Fed an Atherogenic Diet. Circulation 1987;76: I364- I 372.

6. Manuck SB, Adams MR, McCaffery JM, Kaplan JR. Behaviorally Elicited Heart Rate Reactivity and Atherosclerosis in Ovariectomized Cynomolgus Monkeys (Macaca Fascicularis). Arterioscler Thromb Vasc Biol 1997; 17: I7741779.

7. Manuck SB, Kaplan JR, Adams MR, Clarkson TB. Effects of Stress and the Sympathetic Nervous System on Coronary Artery Atherosclerosis in the Cynomolgus Macaque. American heart journal 1988; I 16:328-333.

8. Beere PA, Glagov S, Zarins CK. Retarding Effect of Lowered Heart Rate on Coronary Atherosclerosis. Science 1984;226:180-182.

9. Beere PA, Glagov S, Zarins CK. Experimental Atherosclerosis at the Carotid Bifurcation of the Cynomolgus Monkey. Localization, Compensatory Enlargement, and the Sparing Effect of Lowered Heart Rate. Arteriosclerosis and thrombosis : a journal of vascular biology / American Heart Association 1992; I2: I2451253.

10. Albaladejo P, Carusi A, Apartian A, Lacolley P, Safar ME, Benetos A. Effect of Chronic Heart Rate Reduction with Ivabradine on Carotid and Aortic Structure and Function in Normotensive and Hypertensive Rats. Journal of vascular research 2003;40:320-328.

II. Pei X, Wu B, Tang TY, Gillard JH, Li ZY. Fatigue Crack Growth under Pulsatile Pressure and Plaque Rupture. JACC Cardiovasc Imaging 20।4;7:738-740.

12. Versluis A, Bank AJ, Douglas WH. Fatigue and Plaque Rupture in Myocardial Infarction. J Biomech 2006;39:339347.

13. Virmani R, Kolodgie FD, Burke AP, Finn AV, Gold HK, Tulenko TN et al. Atherosclerotic Plaque Progression and Vulnerability to Rupture: Angiogenesis as a Source of Intraplaque Hemorrhage. Arterioscler Thromb Vasc Biol 2005;25:2054-206I.

14. Michel JB, Virmani R, Arbustini E, Pasterkamp G. Intraplaque Haemorrhages as the Trigger of Plaque Vulnerability. Eur Heart J 201 I;32:1977-1985, 1985a, 1985b, 1985c.

15. Grant EG, Benson CB, Moneta GL, Alexandrov AV, Baker JD, Bluth El et al. Carotid Artery Stenosis: Grayscale and Doppler Ultrasound Diagnosis--Society of Radiologists in Ultrasound Consensus Conference. Ultrasound quarterly 2003; 19:190-198.

16. Chirinos J. Arterial Stiffness: Basic Concepts and Measurement Techniques. J of Cardiovasc Trans Res 20I 2;5:243-255.

17. van Popele NM, Grobbee DE, Bots ML, Asmar R, Topouchian J, Reneman RS et al. Association between Arterial Stiffness and Atherosclerosis: The Rotterdam Study. Stroke 200 I;32:454-460.

18. Meinders JM, Kornet L, Brands PJ, Hoeks AP. Assessment of Local Pulse Wave Velocity in Arteries Using 2d Distension Waveforms. Ultrason Imaging 2001;23:199-2I5.

19. Kwee RM, van Oostenbrugge RJ, Hofstra L, Teule GJ, van Engelshoven JM, Mess WH et al. Identifying Vulnerable Carotid Plaques by Noninvasive Imaging. Neurology 2008;70:240 I-2409.

20. Calcagno C, Cornily JC, Hyafil F, Rudd JHF, Briley-Saebo KC, Mani V et al. Detection of Neovessels in Atherosclerotic Plaques of Rabbits Using Dynamic Contrast Enhanced Mri and I8f-Fdg Pet. Arterioscler Thromb Vasc Biol 2008;28: I3 I I-1317. 
2I. Calcagno C, Vucic E, Mani V, Goldschlager G, Fayad ZA. Reproducibility of Black Blood Dynamic ContrastEnhanced Magnetic Resonance Imaging in Aortic Plaques of Atherosclerotic Rabbits. J Magn Reson Imaging 2010;32:191-198.

22. Vucic E, Calcagno C, Dickson SD, Rudd JH, Hayashi K, Bucerius J et al. Regression of Inflammation in Atherosclerosis by the Lxr Agonist R21 1945: A Noninvasive Assessment and Comparison with Atorvastatin. JACC Cardiovasc Imaging 2012;5:819-828.

23. Vucic E, Dickson SD, Calcagno C, Rudd JH, Moshier E, Hayashi K et al. Pioglitazone Modulates Vascular Inflammation in Atherosclerotic Rabbits Noninvasive Assessment with Fdg-Pet-Ct and Dynamic ContrastEnhanced Mr Imaging. JACC Cardiovasc Imaging 20I I;4: I I00- I 109.

24. Lobatto ME, Fayad ZA, Silvera S, Vucic E, Calcagno C, Mani V et al. Multimodal Clinical Imaging to Longitudinally Assess a Nanomedical Anti-Inflammatory Treatment in Experimental Atherosclerosis. Mol Pharm 20 I 0;7:20202029.

25. Gaens ME, Backes WH, Rozel S, Lipperts M, Sanders SN, Jaspers K et al. Dynamic Contrast-Enhanced Mr Imaging of Carotid Atherosclerotic Plaque: Model Selection, Reproducibility, and Validation. Radiology 2013;266:27I279.

26. Kerwin W, Hooker A, Spilker M, Vicini P, Ferguson M, Hatsukami T et al. Quantitative Magnetic Resonance Imaging Analysis of Neovasculature Volume in Carotid Atherosclerotic Plaque. Circulation 2003; I07:85 I-856.

27. Kerwin WS, O'Brien KD, Ferguson MS, Polissar N, Hatsukami TS, Yuan C. Inflammation in Carotid Atherosclerotic Plaque: A Dynamic Contrast-Enhanced Mr Imaging StudyI. Radiology 2006;24I:459-468.

28. Calcagno C, Ramachandran S, Izquierdo-Garcia D, Mani V, Millon A, Rosenbaum D et al. The Complementary Roles of Dynamic Contrast-Enhanced Mri and I8f-Fluorodeoxyglucose Pet/Ct for Imaging of Carotid Atherosclerosis. European journal of nuclear medicine and molecular imaging 2013;40: I884- I893.

29. Truijman MT, Kwee RM, van Hoof RH, Hermeling E, van Oostenbrugge RJ, Mess WH et al. Combined I8f-Fdg Pet-Ct and Dce-Mri to Assess Inflammation and Microvascularization in Atherosclerotic Plaques. Stroke 2013;44:3568-3570.

30. Wang J, Liu H, Sun J, Xue H, Xie L, Yu S et al. Varying Correlation between I8f-Fluorodeoxyglucose Positron Emission Tomography and Dynamic Contrast-Enhanced Mri in Carotid Atherosclerosis: Implications for Plaque Inflammation. Stroke 20।44;5: I842-I845.

31. Moreno PR, Purushothaman K-R, Sirol M, Levy AP, Fuster V. Neovascularization in Human Atherosclerosis. Circulation 2006; I I3:2245-2252.

32. DiFrancesco D, Camm JA. Heart Rate Lowering by Specific and Selective I(F) Current Inhibition with Ivabradine: A New Therapeutic Perspective in Cardiovascular Disease. Drugs 2004;64: I 757- 1765.

33. Bois P, Bescond J, Renaudon B, Lenfant J. Mode of Action of Bradycardic Agent, S I6257, on lonic Currents of Rabbit Sinoatrial Node Cells. British journal of pharmacology 1996; I 18:105 I-1057.

34. Gardiner SM, Kemp PA, March JE, Bennett T. Acute and Chronic Cardiac and Regional Haemodynamic Effects of the Novel Bradycardic Agent, SI6257, in Conscious Rats. British journal of pharmacology I 995; I I 5:579-586.

35. Custodis F, Baumhakel M, Schlimmer N, List F, Gensch C, Bohm M et al. Heart Rate Reduction by Ivabradine Reduces Oxidative Stress, Improves Endothelial Function, and Prevents Atherosclerosis in Apolipoprotein E-Deficient Mice. Circulation 2008; I 17:2377-2387.

36. Lobbes MB, Miserus RJ, Heeneman S, Passos VL, Mutsaers PH, Debernardi N et al. Atherosclerosis: ContrastEnhanced Mr Imaging of Vessel Wall in Rabbit Model--Comparison of Gadofosveset and Gadopentetate Dimeglumine. Radiology 2009;250:682-69।.

37. Phinikaridou A, Ruberg FL, Hallock KJ, Qiao Y, Hua N, Viereck J et al. In Vivo Detection of Vulnerable Atherosclerotic Plaque by Mri in a Rabbit Model. Circ Cardiovasc Imaging 20 I 0;3:323-332.

38. Hoeks AP, Brands PJ, Smeets FA, Reneman RS. Assessment of the Distensibility of Superficial Arteries. Ultrasound Med Biol 1990; 16:121-128. 
39. Brands PJ, Hoeks AP, Ledoux LA, Reneman RS. A Radio Frequency Domain Complex Cross-Correlation Model to Estimate Blood Flow Velocity and Tissue Motion by Means of Ultrasound. Ultrasound Med Biol 1997;23:9 I I920.

40. Yankeelov TE, Luci JJ, Lepage M, Li R, Debusk L, Lin PC et al. Quantitative Pharmacokinetic Analysis of Dce-Mri Data without an Arterial Input Function: A Reference Region Model. Magn Reson Imaging 2005;23:5 I 9-529.

4I. Jaspers K, Leiner T, Dijkstra P, Oostendorp M, van Golde JM, Post MJ et al. Optimized Pharmacokinetic Modeling for the Detection of Perfusion Differences in Skeletal Muscle with Dce-Mri: Effect of Contrast Agent Size. Med Phys 2010;37:5746-5755.

42. Dominguez-Rodriguez A, Blanco-Palacios G, Abreu-Gonzalez P. Increased Heart Rate and Atherosclerosis: Potential Implications of Ivabradine Therapy. World journal of cardiology 20 I I;3: I 0 I- 104.

43. Tardif J-C. Heart Rate as a Treatable Cardiovascular Risk Factor. British Medical Bulletin 2009;90:7I-84.

44. Sun J, Canton G, Balu N, Hippe DS, Xu D, Liu J et al. Blood Pressure Is a Major Modifiable Risk Factor Implicated in Pathogenesis of Intraplaque Hemorrhage: An in Vivo Magnetic Resonance Imaging Study. Arterioscler Thromb Vasc Biol 2016; 10.1 I6I /atvbaha. I 15.307043.

45. Sluimer JC, Gasc JM, van Wanroij JL, Kisters N, Groeneweg M, Sollewijn Gelpke MD et al. Hypoxia, HypoxiaInducible Transcription Factor, and Macrophages in Human Atherosclerotic Plaques Are Correlated with Intraplaque Angiogenesis. J Am Coll Cardiol 2008;5।:I258-1265.

46. Getz GS, Reardon CA. Animal Models of Atherosclerosis. Arterioscler Thromb Vasc Biol 201 2;32:I I 04-I I I 5.

47. Kapourchali FR, Surendiran G, Chen L, Uitz E, Bahadori B, Moghadasian MH. Animal Models of Atherosclerosis. World journal of clinical cases 20|4;2: I26-I32.

48. Millon A, Canet-Soulas E, Boussel L, Fayad Z, Douek P. Animal Models of Atherosclerosis and Magnetic Resonance Imaging for Monitoring Plaque Progression. Vascular 2014;22:221-237.

49. Niccoli G, Borovac JA, Vetrugno V, Camici PG, Crea F. Ivabradine in Acute Coronary Syndromes: Protection Beyond Heart Rate Lowering. International journal of cardiology 2017;10.1016/j.ijcard.2017.02.046.

50. Swedberg K, Komajda M, Bohm M, Borer JS, Ford I, Dubost-Brama A et al. Ivabradine and Outcomes in Chronic Heart Failure (Shift): A Randomised Placebo-Controlled Study. Lancet 2010;376:875-885.

5I. Fox K, Ford I, Steg PG, Tardif JC, Tendera M, Ferrari R et al. Ivabradine in Stable Coronary Artery Disease without Clinical Heart Failure. N Engl J Med 2014;37I: I091- I099.

52. Nawarskas JJ, Bowman BN, Anderson JR. Ivabradine: A Unique and Intriguing Medication for Treating Cardiovascular Disease. Cardiol Rev 20I5;23:20I-2II.

53. Oliphant CS, Owens RE, Bolorunduro OB, Jha SK. Ivabradine: A Review of Labeled and Off-Label Uses. Am J Cardiovasc Drugs 2016;16:337-347.

54. Lees-Miller JP, Guo J, Wang Y, Perissinotti LL, Noskov SY, Duff HJ. Ivabradine Prolongs Phase 3 of Cardiac Repolarization and Blocks the Hergl (Kenh2) Current over a Concentration-Range Overlapping with That Required to Block Hcn4. J Mol Cell Cardiol 2015;85:7I-78.

55. Calcagno C, Lobatto ME, Dyvorne H, Robson PM, Millon A, Senders ML et al. Three-Dimensional Dynamic Contrast-Enhanced Mri for the Accurate, Extensive Quantification of Microvascular Permeability in Atherosclerotic Plaques. NMR Biomed 2015;28:I304-1314.

56. Epstein FH. Mr in Mouse Models of Cardiac Disease. NMR Biomed 2007;20:238-255.

57. Zuurbier CJ, Emons VM, Ince C. Hemodynamics of Anesthetized Ventilated Mouse Models: Aspects of Anesthetics, Fluid Support, and Strain. Am J Physiol Heart Circ Physiol 2002;282:H2099-2 105.

58. Albrecht M, Henke J, Tacke S, Markert M, Guth B. Effects of Isoflurane, Ketamine-Xylazine and a Combination of Medetomidine, Midazolam and Fentanyl on Physiological Variables Continuously Measured by Telemetry in Wistar Rats. BMC Vet Res 2014;10:198. 



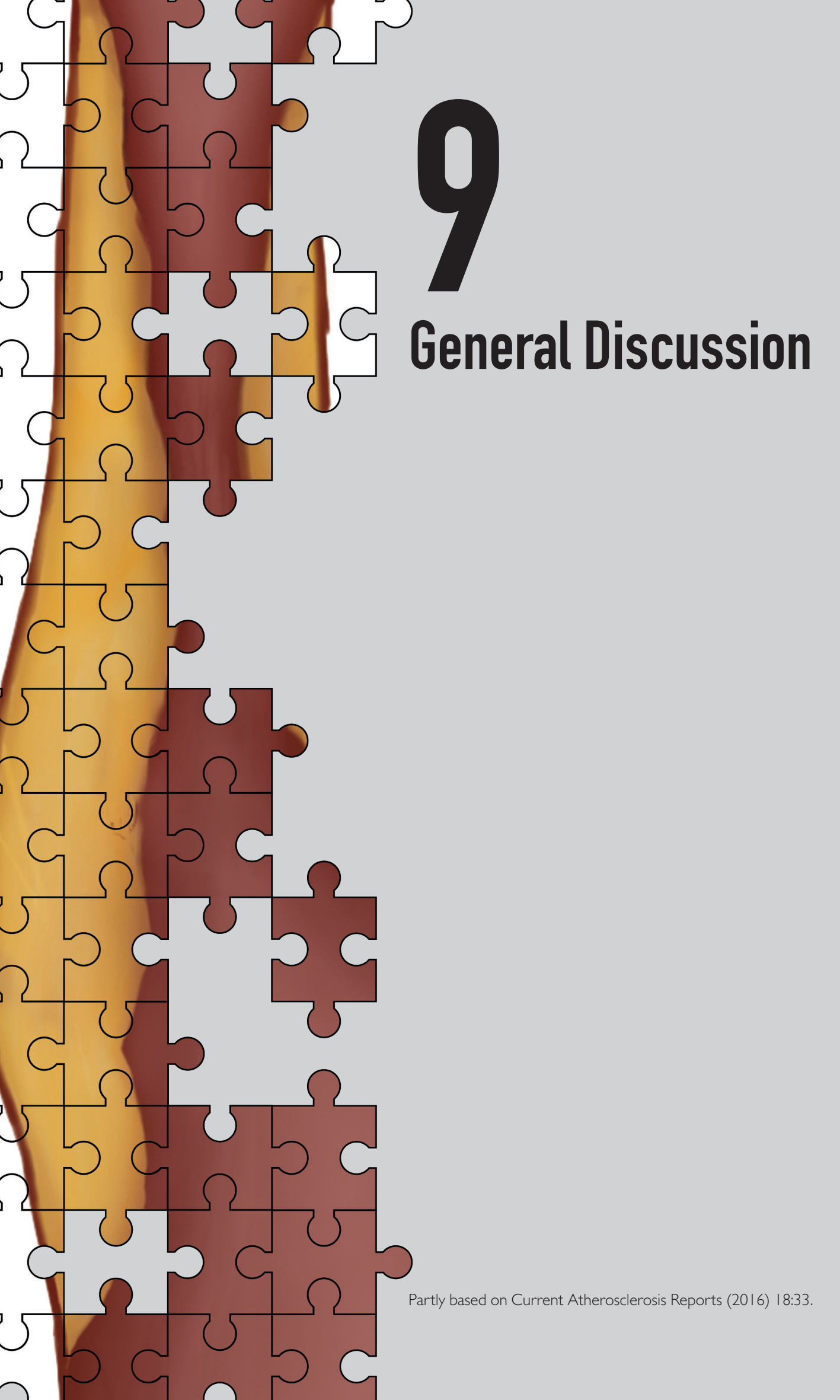


Currently the guidelines for treatment of patients with a stenosis of the carotid artery are solely based on the degree of luminal stenosis and symptomatology (I). However, research over the past decades has shown that the atherosclerotic plaque composition is associated with (future) cerebrovascular events (2-5). Large trials have shown that symptomatic patients with a $70-99 \%$ carotid stenosis benefit from surgical removal of the atherosclerotic plaque (carotid endarterectomy, CEA) with a number needed to treat (NNT) of 6. However, for patients with a 50-69\% stenosis the NNT increases to 22 (6). Previous histological studies have shown a higher incidence of specific characteristics of atherosclerotic plaques in CEA specimens from symptomatic patients compared to asymptomatic patients (7-II). Due to technical improvements of non-invasive imaging methods, interest in identification of these "vulnerable plaques" with non-invasive imaging has increased greatly over the past decade. Identification of patients with a "vulnerable plaque" that may improve selection of patients that will benefit from CEA.

Features of a vulnerable atherosclerotic plaque are lipid-rich necrotic core (LRNC) with a thin or ruptured fibrous cap (TRFC), presence of inflammatory cells, ulcerations, and intraplaque hemorrhage (IPH) (I2). Next to these features, an increased plaque microvasculature has been suggested as an important marker of plaque vulnerability (13). The plaque microvasculature can be studied non-invasively using dynamic contrast-enhanced magnetic resonance imaging (DCE-MRI) (I4).

Recent DCE-MRI studies have suggested an association between plaque microvasculature and plaque inflammation $(15,16)$ and the presence of IPH $(17)$. Calcagno et al. (I5) have shown a weak link between plaque inflammation and plaque microvasculature. However, results from Wang et al. (16) showed that the correlation between the plaque inflammation and microvasculature varied depending on clinical condition (16). A positive association between the plaque microvasculature and the presence of intraplaque hemorrhage has been reported by Sun et al. (17). These studies have shown that the plaque microvasculature may provide additional information compared to other vulnerable plaque features. Investigation of the plaque microvasculature may also provide further insight into pathological mechanisms involved in plaque development and destabilization.

The hypothesis of the present thesis was that non-invasive imaging of the plaque microvasculature with DCE-MRI may provide further insight in the atherosclerotic process. For this hypothesis, several experimental studies were performed. In these studies, symptomatic patients with carotid atherosclerosis underwent MRI examination. Additionally a preclinical study in atherosclerotic rabbits was performed. With these studies, the following objectives were addressed:

- To further improve and validate DCE-MRI methodology

- To investigate the association between plaque microvasculature and important features of plaque vulnerability (plaque inflammation and presence of intraplaque hemorrhage)

- To explore whether plaque microvasculature is related to the type of cerebrovascular symptoms in patients with mild to moderate carotid stenosis 
- To evaluate if DCE-MRI can be used as evaluation tool to investigate the effect of a heartrate reducing therapy on features of plaque vulnerability

\section{STANDARDIZATION OF ACQUISITION AND ANALYSIS METHODS}

Analysis of DCE-MRI data can be performed using semi-quantitative and quantitative methods. Of these two, semi-quantitative analysis using the area-under-the-curve (AUC) is easy to implement. However, AUC reflects the amount of contrast medium delivered to and retained by the tissue for a given period, but does not reflect a specific physiological parameter (18). Quantitative analysis using two-compartment models allows the determination of pharmacokinetic parameters reflecting the amount, flow, and leakiness of the microvasculature.

To further advance DCE-MRI so that it may be used in daily clinical practice, more uniform acquisition and analysis methods should be agreed upon. Previous studies have shown that DCE-MRI derived parameters are influenced by the contrast medium (19) and the pharmacokinetic model that is being used (20), making direct cross-study comparisons difficult. Results from the current thesis show that the vascular input function (VIF) (chapter 3) and analysed vascular region (chapter 4 ) also influence absolute values of the pharmacokinetic parameters. In chapter 3, it is shown that the magnitude-based VIF is influenced by the local blood flow velocity, leading to an underestimation of the contrast medium concentration. Results from chapter 4 show that descriptive statistics (mean, median, and $75^{\text {th }}$ percentile) from a single vascular region differ. Additionally, a significantly increased median adventitial $\mathrm{K}^{\text {trans }}$ compared to entire vessel wall $\mathrm{K}^{\text {trans }}$ was found, although the correlation coefficients with histological measurements of plaque microvasculature from these two regions are similar. Since during CEA only the intima and (part of) the media are surgically removed, these results suggest a link between the microvasculature of the atherosclerotic plaque and the originating adventitial microvessels. The results from the current thesis and previous studies show that use of a standard imaging and data analysis protocol is essential, in particular for longitudinal studies of plaque microvasculature and for determination of risk thresholds in the future. These findings are also important for direct comparisons of absolute values in different studies, since most studies only reported results from a single region of the vascular wall. Despite the strong correlation between pharmacokinetic parameters from the vessel wall and adventitia in symptomatic patients scheduled for CEA, it remains to be determined whether these results can be translated to other patient groups. It is an interesting research question whether the vessel wall and adventitial microvasculature equally contribute to the various processes of atherosclerosis or whether these contributions differ.

In the present thesis, DCE-MRI analysis was performed using custom in-house build software in which manual segmentation of the atherosclerotic plaque was required. For the implementation of DCE-MRI in large multicenter trials and ultimately clinical routine, development of automated analysis software would be beneficial. There has been initial research into automated analysis (2I), but current research on automated software for analysis of the carotid plaque focuses mainly on quantification of the plaque components on anatomic MR images (22-24). A future goal for carotid plaque analysis software would be the incorporation of DCE-MRI analysis in a quick, robust, and user friendly way. 


\section{ASSOCIATION OF PLAQUE MICROVASCULATURE WITH OTHER FEATURES OF PLAQUE VULNERABILITY}

The atherosclerotic plaque microvasculature is thought to be related to several other features of plaque vulnerability. During the development and progression of atherosclerosis inflammatory cells play an important role (25). Activated macrophages induce hypoxia within the atherosclerotic plaque due to their high metabolic rate (26). Hypoxia stimulates the development of new microvessels, originating from the adventitia, the outer layer of the vessel wall (27). In general, these angiogenic microvessels have a low endothelial integrity, potentially leading to extravasation of inflammatory cells and erythrocytes from the microvessel lumen into plaque tissue (27). Extravasation of erythrocytes is considered to be an important factor in the development of IPH (28). Since the erythrocyte membranes constitute for forty percent of lipids (28), extravasation of erythrocytes leads to increased cholesterol deposition in the plaque tissue, stimulating the further recruitment of inflammatory cells. Thus (leaky) plaque microvasculature is thought to be a key feature in plaque destabilization (29).

Varying associations between plaque microvasculature (measured using DCE-MRI) and plaque inflammation (measured by ${ }^{18} \mathrm{~F}-\mathrm{FDG}$ uptake or macrophage content) have been published $(15,16)$. Results have show a significant inverse relationship between plaque perfusion $\left(\mathrm{K}^{\text {trans }}\right)$ and plaque inflammation measured with ${ }^{18}$ fluorine-fluorodeoxyglucose $\left({ }^{18} \mathrm{~F}-\mathrm{FDG}\right)$ positron emission tomography/computed tomography (PET-CT) $(\rho=-0.24, p<0.05)$ in patients with coronary heart disease (CHD) or CHD risk equivalent and a carotid plaque with target-to-background-ratio (TBR) $\geq 1.6$ on ${ }^{18} \mathrm{~F}-\mathrm{FDG}$ PET-CT (I5). A second study of patients with carotid plaque (16) found that the correlation between the microvasculature and inflammation depend on the patients' clinical condition. Within this study, a weak, marginal non-significant correlation (Spearman $\rho=0.22, p=0.068$ ) was found for all carotid plaques (both symptomatic and asymptomatic). A significant correlation between $K^{\text {trans }}$ and TBR (Spearman $\rho=0.59, p=0.006$ ) was found for symptomatic carotid plaques, which was not found for asymptomatic plaques (Spearman $\rho=0.07, p=0.625$ ). In the present thesis (chapter 6), the association between plaque inflammation and microvasculature was investigated in symptomatic patients with a mild to moderate stenosis. Results show a weak positive correlation (Spearman's $\rho=0.3, p=0.035)$ between plaque inflammation ( ${ }^{18} \mathrm{~F}-\mathrm{FDG}$ uptake) and microvasculature $\left(K^{\text {trans }}\right)$ indicating that the imaging modalities may provide complimentary risk markers.

The association between the plaque microvasculature and the presence of IPH has been studied non-invasively using DCE-MRI by Sun et al. (17). Results from this study showed a positive association between $\mathrm{K}^{\text {trans }}$ from the adventitia and the presence of IPH in 27 patients with carotid plaque. The aim of the study in chapter 5 of the present thesis was to further investigate the potential positive association between the atherosclerotic plaque microvasculature and presence of $\mathrm{IPH}$ in a relatively large prospective cohort study of patients $(n=|0|)$ with $\geq 2$ $\mathrm{mm}$ carotid plaque. Results from this study showed no difference in median adventitial $\mathrm{K}^{\text {trans }}$ for patients with and without IPH, while a decreased median $\mathrm{K}^{\text {trans }}$ from the entire vessel wall was found in the presence of IPH. Therefore these results indicate that no ongoing leakage of erythrocytes from the plaque microvasculature occurs several weeks after a cerebrovascular event in plaques with IPH. This suggests that not only plaque microvasculature, but additional 
factors, such as a disrupted plaque surface, may contribute to the development of IPH. In order to unravel the underlying mechanisms contributing to IPH, further longitudinal studies are warranted.

Currently, studies have focused on the association between the plaque microvasculature and either plaque inflammation or the presence of IPH. For future studies, it would be interesting to study the interplay between plaque inflammation, presence of $\mathrm{PH}$, a thin/ruptured fibrous cap, and plaque microvasculature combined in a single study. The recent introduction of hybrid PET-MRI systems provides excellent opportunities for this purpose using a single imaging system. Recent research (30-34) has already shown the potential of hybrid PET-MR systems for the imaging of atherosclerosis. However, the additional value of DCE-MRI in PET-MR imaging is yet to be explored.

\section{POTENTIAL OF DCE-MRI TO PREDICT CEREBROVASCULAR ISCHEMIC EVENTS}

In chapter 7 of this thesis, a positive association between the adventitial microvasculature $\left(K^{\text {trans }}\right)$ and the type of recent cerebrovascular events was found. Patients with a recent history of stroke show an increased adventitial $\mathrm{K}^{\text {trans }}$ compared to patients with a recent transient ischemic attack (TIA) or ocular TIA (also known as amaurosis fugax), independent of clinical risk factors. The results of the observational study described in the present thesis need to be confirmed in a prospective longitudinal study investigating the plaque microvasculature of asymptomatic and symptomatic patients in relation to future clinical symptoms. This investigation may provide additional information on the prospective value of DCE-MRI in risk stratification of patients. Large epidemiological studies have already shown a number of clinical risk factors that are associated with ischemic stroke, though these studies did not report differences in risk factors for patients based on the type of clinically diagnosed event (e.g. (ocular) TIA versus stroke patients) (35-40). With regard to carotid atherosclerosis, currently studies have focused on differences of plaque characteristics between symptomatic and asymptomatic patients. These studies have shown a higher incidence of vulnerable plaque characteristics (e.g. a thin or ruptured FC, presence of IPH, and LRNC) (2-5,4I-44) in symptomatic patients. Within these studies, the plaque microvasculature was not investigated since no DCE-MRI was performed. Therefore, within these studies, the association between clinical symptoms and the plaque microvasculature was not investigated.

Up to now, research of the plaque microvasculature has focused on symptomatic patients with a recent history of cerebrovascular events and only a small number of studies have been performed in asymptomatic patients $(16,45)$. One study (16) showed a non-significant correlation between $K^{\text {trans }}$ and TBR in asymptomatic patients $(\rho=0.07, p=0.625)$. A second study (45), performed in "Ground Zero" workers showed an increased $K^{\text {trans }}$ in people that were exposed to high levels of particulate matter compared to workers with a low exposure. 
Non-invasive quantification of the plaque microvasculature in asymptomatic patients may provide further information on the potential added value of non-invasive imaging of the plaque microvasculature compared to other plaque features.

Whether an increased microvasculature of the carotid artery is related to the presence of infarcts on brain MR images remains to be determined. For this purpose, it is important to be distinguish recent from old infarcts. MRI is able to distinguish old and recent infarctions with the use of fluid-attenuated inversion recovery (FLAIR) and diffusion-weighted imaging (DWI) acquisitions. Recent infarctions remain positive on DWI until 2 I days after the ischemic event. Therefore, it is essential that imaging is performed within this time frame to provide accurate information for investigation of the relationship between plaque microvasculature and the presence of (recent) infarcts on MRI. The admission of patients to a stroke unit for a period of 24 hours to monitor their cardiac rhythm may provide opportunities to perform the proposed investigation.

The potential predictive value of DCE-MRI for plaque progression or development of vulnerable plaque features is of great interest and remains to be determined, and its predictive value for cerebrovascular ischemic events needs to be investigated in a prospective clinical trial.

\section{Potential Role of PLAQUe biomechanics IN THE PROCESS OF PLAQUE DESTABILIZATION}

The results of a preclinical rabbit study described in chapter 8 showed that a heart rate reducing therapy results in a decrease in macrophage content and a reduced dynamic contrast-enhancement on dynamic MR images (reduced AUC and $\mathrm{K}^{\text {trans }}$ ). The reduction of both parameters indicated a reduction in (leaky) plaque microvasculature. Histological analysis showed no differences in the microvessel density between treated and non-treated rabbits. In addition, a significant correlation was found between the semi-quantitative DCE-MRI parameter AUC with the microvessel density, but not with $\mathrm{K}^{\text {trans }}$, a reflection of the microvascular flow, density, and permeability. Together this suggests a reduction in the plaque microvascular leakiness. The reduction of the microvascular leakiness may be due to a reduction of the shear and/ or tensile stress on the atherosclerotic plaque caused by the reduced heart-rate. Both shear and tensile stress have been suggested to induce endothelial damage, leading to an increased endothelial permeability to inflammatory mediators of the endothelium $(46,47)$. Alternatively, the treatment may have reduced microvessel rupture, since it was previously suggested that pressure gradients within the plaque that occur during every heart beat lead to microvessel rupture (48).

The preclinical study discussed in the present thesis shows the ability of DCE-MRI as an evaluation tool for potential therapies in the treatment of atherosclerosis. This is in line with previous animal and patient studies showing that DCE-MRI can be employed to investigate the effect potential treatments of atherosclerosis and resulting changes in the plaque microvasculature (49-52). 
Results from this thesis suggest that plaque biomechanics and deformation may play an important role in the development of vulnerable plaque features. To further investigate the potential relationship between the plaque microvasculature and plaque biomechanics, longitudinal studies incorporating both measurements may be performed. These studies may provide additional information on the role of plaque biomechanics on the plaque microvasculature in the process of plaque formation and destabilization. Measurement of the plaque deformation may be performed using a duplex ultrasound examination, which is a reliable, low-cost technique commonly used in clinical routine. Alternatively, measurement of plaque deformation may be performed using MRI (53). Despite being a technically challenging technique due to spatial and temporal requirements, strain imaging of the carotid artery using MRI may provide estimates of plaque strain or mechanical properties of the atherosclerotic lesion.

\section{Alternative future Perspectives of DCE-MRI}

\section{Developments of DCE-MRI acquisition methods}

In the current thesis and the majority of studies reported in literature, either bright or black blood acquisition techniques have been employed for DCE-MRI of atherosclerosis. Both these acquisition techniques have their strengths and weaknesses. While bright blood techniques allow quantification of the contrast medium concentration in the vascular lumen (the vascular input function), it is challenging to delineate the lumen from the vessel wall in bright blood MRI. The recent introduction of interleaved acquisition methods (54), providing both bright and black blood images, may be an important step towards combining the strength and weaknesses of both acquisitions methods, enabling determination of an individualized vascular input function and accurate delineation of the vessel lumen from the vessel wall. Additionally, the recent introduction of 3D acquisition techniques (55) may provide increased spatial information as compared to the in this thesis and in literature currently employed 2D techniques, although at the expense of temporal resolution. Currently, these 3D acquisition techniques have only been explored in preclinical rabbit studies and therefore their potential in clinical studies remains to be determined.

All clinical DCE-MRI studies to date have been performed at I.5 and 3.0 Tesla. The potential of carotid MRI at 7.0 T has already been explored $(56,57)$, and results show a potential increase in signal-to-noise ratio (SNR) due to the increased field strength. However, the increase in SNR may be diminished by increased relaxivity of the contrast medium. The potentially increased SNR at 7.0 T would allow increased spatial and/or temporal resolution, but these studies also demonstrate that further technical developments are required to enable complete plaque characterization. 


\section{Clinical studies using DCE-MRI of the vessel wall in other vascular beds}

The potential of DCE-MRI for quantification of the plaque microvasculature can be extended to other (human) vascular territories beyond the carotid artery. Indeed, the potential use of DCE-MRI to study microvasculature in the aortic wall of abdominal aortic aneurysms has already been explored $(58,59)$. Results of these studies have shown that measurements are reproducible with a high technical success rate. The Patlak model provided significantly lower relative fit uncertainties with comparable scan-rescan reproducibilities compared to other pharmacokinetic models (Tofts and extended Tofts). Therefore, the Patlak model was the most suited pharmacokinetic model. In the future, more studies are warranted to investigate the predictive potential of DCE-MRI derived parameters for abdominal aortic aneurysm rupture risk. Development of DCE-MRI protocols to study the microvasculature of coronary arteries may provide further insight in the role of plaque microvasculature in myocardial infarction. However, the development of such DCE-MRI protocols may provide technical challenges due to cardiac motion.

\section{CONCLUSION}

The aim of the current thesis was to investigate how non-invasive imaging of the plaque microvasculature with DCE-MRI may be used to gain further insight in the atherosclerotic process. Over the past decade, DCE-MRI has developed from a novel imaging tool to a useful non-invasive research tool used in animal and patient studies of plaque microvasculature. Within the current thesis and other studies, DCE-MRI has been used to investigate the relationship between plaque microvasculature and other plaque features such as inflammation and intraplaque hemorrhage, for assessing effectiveness of therapeutic interventions, and in the potential of plaque microvasculature in relation to the identification of patients with increased cardiovascular risks. Future studies could apply DCE-MRI to elucidate mechanisms that contribute to plaque progression and destabilization, specifically the interplay between inflammation, increased microvasculature, and intraplaque hemorrhage. Also of great interest is the potential predictive value of plaque microvasculature DCE-MRI for plaque progression and (severity of) future cerebrovascular ischemic events (such as stroke). 


\section{REFERENCES}

I. Neurologie NVv. Richtlijn Diagnostiek, Behandeling En Zorg Voor Patiënten Met Een Beroerte. 2008.

2. Gupta A, Baradaran H, Schweitzer AD, Kamel H, Pandya A, Delgado D et al. Carotid Plaque Mri and Stroke Risk: A Systematic Review and Meta-Analysis. Stroke 2013;44:307I-3077.

3. Hosseini AA, Kandiyil N, Macsweeney ST, Altaf N, Auer DP. Carotid Plaque Hemorrhage on Magnetic Resonance Imaging Strongly Predicts Recurrent Ischemia and Stroke. Ann Neurol 2013;73:774-784.

4. Saam T, Hetterich H, Hoffmann V, Yuan C, Dichgans M, Poppert H et al. Meta-Analysis and Systematic Review of the Predictive Value of Carotid Plaque Hemorrhage on Cerebrovascular Events by Magnetic Resonance Imaging. J Am Coll Cardiol 2013;62:1081-109I.

5. Yuan C, Zhang SX, Polissar NL, Echelard D, Ortiz G, Davis JW et al. Identification of Fibrous Cap Rupture with Magnetic Resonance Imaging Is Highly Associated with Recent Transient Ischemic Attack or Stroke. Circulation 2002; 105: 181-185.

6. Rothwell PM, Eliasziw M, Gutnikov SA, Fox AJ, Taylor DW, Mayberg MR et al. Analysis of Pooled Data from the Randomised Controlled Trials of Endarterectomy for Symptomatic Carotid Stenosis. Lancet 2003;36 I: I07-I I 6.

7. Spagnoli LG, Mauriello A, Sangiorgi G, Fratoni S, Bonanno E, Schwartz RS et al. Extracranial Thrombotically Active Carotid Plaque as a Risk Factor for Ischemic Stroke. JAMA : the journal of the American Medical Association 2004;292: 1845-1852.

8. Redgrave JN, Lovett JK, Gallagher PJ, Rothwell PM. Histological Assessment of 526 Symptomatic Carotid Plaques in Relation to the Nature and Timing of Ischemic Symptoms: The Oxford Plaque Study. Circulation 2006; I 13:2320-2328.

9. Park AE, McCarthy WJ, Pearce WH, Matsumura JS, Yao JS. Carotid Plaque Morphology Correlates with Presenting Symptomatology. J Vasc Surg 1998;27:872-878; discussion 878-879.

10. Ballotta E, Da Giau G, Renon L. Carotid Plaque Gross Morphology and Clinical Presentation: A Prospective Study of 457 Carotid Artery Specimens. J Surg Res 2000;89:78-84.

II. Gao P, Chen ZQ, Bao YH, Jiao LQ, Ling F. Correlation between Carotid Intraplaque Hemorrhage and Clinical Symptoms: Systematic Review of Observational Studies. Stroke 2007;38:2382-2390.

12. Naghavi M, Libby P, Falk E, Casscells SW, Litovsky S, Rumberger J et al. From Vulnerable Plaque to Vulnerable Patient: A Call for New Definitions and Risk Assessment Strategies: Part I. Circulation 2003; I08: I664- I672.

13. Moreno PR, Purushothaman KR, Fuster V, Echeverri D, Truszczynska H, Sharma SK et al. Plaque Neovascularization Is Increased in Ruptured Atherosclerotic Lesions of Human Aorta: Implications for Plaque Vulnerability. Circulation 2004; I 10:2032-2038.

14. Calcagno C, Mani V, Ramachandran S, Fayad ZA. Dynamic Contrast Enhanced (Dce) Magnetic Resonance Imaging (Mri) of Atherosclerotic Plaque Angiogenesis. Angiogenesis 2010;13:87-99.

15. Calcagno C, Ramachandran S, Izquierdo-Garcia D, Mani V, Millon A, Rosenbaum D et al. The Complementary Roles of Dynamic Contrast-Enhanced Mri and I8f-Fluorodeoxyglucose Pet/Ct for Imaging of Carotid Atherosclerosis. European journal of nuclear medicine and molecular imaging 20। 3;40: I884- I893.

16. Wang J, Liu H, Sun J, Xue H, Xie L, Yu S et al. Varying Correlation between I8f-Fluorodeoxyglucose Positron Emission Tomography and Dynamic Contrast-Enhanced Mri in Carotid Atherosclerosis: Implications for Plaque Inflammation. Stroke 2014;45: I842- I845.

17. Sun J, Song Y, Chen H, Kerwin WS, Hippe DS, Dong L et al. Adventitial Perfusion and Intraplaque Hemorrhage: A Dynamic Contrast-Enhanced Mri Study in the Carotid Artery. Stroke 2013;44:I03 I- 036.

18. Walker-Samuel S, Leach MO, Collins DJ. Evaluation of Response to Treatment Using Dce-Mri: The Relationship between Initial Area under the Gadolinium Curve (laugc) and Quantitative Pharmacokinetic Analysis. Phys Med Biol 2006;51:3593-3602.

19. Kerwin WS, Zhao X, Yuan C, Hatsukami TS, Maravilla KR, Underhill HR et al. Contrast-Enhanced Mri of Carotid Atherosclerosis: Dependence on Contrast Agent. Journal of Magnetic Resonance Imaging 2009;30:35-40. 
20. Gaens ME, Backes WH, Rozel S, Lipperts M, Sanders SN, Jaspers K et al. Dynamic Contrast-Enhanced Mr Imaging of Carotid Atherosclerotic Plaque: Model Selection, Reproducibility, and Validation. Radiology 20I3;266:27I-279.

21. Kerwin WS, Oikawa M, Yuan C, Jarvik GP, Hatsukami TS. Mr Imaging of Adventitial Vasa Vasorum in Carotid Atherosclerosis. Magn Reson Med 2008;59:507-5।4.

22. Kerwin W, Xu D, Liu F, Saam T, Underhill H, Takaya N et al. Magnetic Resonance Imaging of Carotid Atherosclerosis: Plaque Analysis. Top Magn Reson Imaging 2007; 18:37I-378.

23. van Engelen A. Multimodal Image Analysis for Carotid Artery Plaque Characterization. Rotterdam: Erasmus University Rotterdam; 2014.

24. Yoneyama T, Sun J, Hippe DS, Balu N, Xu D, Kerwin WS et al. In Vivo Semi-Automatic Segmentation of Multicontrast Cardiovascular Magnetic Resonance for Prospective Cohort Studies on Plaque Tissue Composition: Initial Experience. Int J Cardiovasc Imaging 2016;32:73-8I.

25. Moore KJ, Tabas I. Macrophages in the Pathogenesis of Atherosclerosis. Cell 20 I ; | 45:34I-355.

26. Sluimer JC, Gasc JM, van Wanroij JL, Kisters N, Groeneweg M, Sollewijn Gelpke MD et al. Hypoxia, HypoxiaInducible Transcription Factor, and Macrophages in Human Atherosclerotic Plaques Are Correlated with Intraplaque Angiogenesis. J Am Coll Cardiol 2008;5।:I258-1265.

27. Sluimer JC, Daemen MJ. Novel Concepts in Atherogenesis: Angiogenesis and Hypoxia in Atherosclerosis. J Pathol 2009;21 8:7-29.

28. Kolodgie FD, Gold HK, Burke AP, Fowler DR, Kruth HS, Weber DK et al. Intraplaque Hemorrhage and Progression of Coronary Atheroma. N EngI J Med 2003;349:23 I6-2325.

29. Moreno PR, Purushothaman K-R, Sirol M, Levy AP, Fuster V. Neovascularization in Human Atherosclerosis. Circulation 2006; I I3:2245-2252.

30. Hyafil F, Schindler A, Sepp D, Obenhuber T, Bayer-Karpinska A, Boeckh-Behrens T et al. High-Risk Plaque Features Can Be Detected in Non-Stenotic Carotid Plaques of Patients with Ischaemic Stroke Classified as Cryptogenic Using Combined F-Fdg Pet/Mr Imaging. European journal of nuclear medicine and molecular imaging 20I5;10.1007/s00259-0I5-320I-8.

31. Rischpler C, Nekolla SG, Beer AJ. Pet/Mr Imaging of Atherosclerosis: Initial Experience and Outlook. Am J Nucl Med Mol Imaging 2013;3:393-396.

32. Ripa RS, Kjaer A. Imaging Atherosclerosis with Hybrid Positron Emission Tomography/Magnetic Resonance Imaging. Biomed Res Int 2015;2015:914516.

33. Ripa RS, Knudsen A, Hag AM, Lebech AM, Loft A, Keller SH et al. Feasibility of Simultaneous Pet/Mr of the Carotid Artery: First Clinical Experience and Comparison to Pet/Ct. Am J Nucl Med Mol Imaging 20I 3;3:36I371 .

34. Bini J, Eldib M, Robson PM, Calcagno C, Fayad ZA. Simultaneous Carotid Pet/Mr: Feasibility and Improvement of Magnetic Resonance-Based Attenuation Correction. Int J Cardiovasc Imaging 20I5; I0. I007/s I0554-0I5$066 \mathrm{I}-7$.

35. Appelros P, Stegmayr B, Terent A. Sex Differences in Stroke Epidemiology: A Systematic Review. Stroke 2009;40: 1082-1090.

36. Arboix A. Cardiovascular Risk Factors for Acute Stroke: Risk Profiles in the Different Subtypes of Ischemic Stroke World journal of clinical cases 2015;3:4I8-429.

37. MacMahon S, Peto R, Cutler J, Collins R, Sorlie P, Neaton J et al. Blood Pressure, Stroke, and Coronary Heart Disease. Part I, Prolonged Differences in Blood Pressure: Prospective Observational Studies Corrected for the Regression Dilution Bias. Lancet 1990;335:765-774.

38. Jarrett RJ. Epidemiology and Public Health Aspects of Non-Insulin-Dependent Diabetes Mellitus. Epidemiol Rev 1989; II:|5|-|7|.

39. Higa M, Davanipour Z. Smoking and Stroke. Neuroepidemiology I99।;10:21 I-222. 
40. Suk SH, Sacco RL, Boden-Albala B, Cheun JF, Pittman JG, Elkind MS et al. Abdominal Obesity and Risk of Ischemic Stroke: The Northern Manhattan Stroke Study. Stroke 2003;34: I586- I 592.

4I. Kwee RM, van Oostenbrugge RJ, Mess WH, Prins MH, van der Geest RJ, ter Berg JWM et al. Mri of Carotid Atherosclerosis to Identify Tia and Stroke Patients Who Are at Risk of a Recurrence. Journal of Magnetic Resonance Imaging 2012;10.1002/jmri.23918:n/a-n/a.

42. Takaya N, Yuan C, Chu B, Saam T, Underhill H, Cai J et al. Association between Carotid Plaque Characteristics and Subsequent Ischemic Cerebrovascular Events: A Prospective Assessment with Mri--Initial Results. Stroke 2006;37:818-823.

43. Altaf N, Daniels L, Morgan PS, Auer D, MacSweeney ST, Moody AR et al. Detection of Intraplaque Hemorrhage by Magnetic Resonance Imaging in Symptomatic Patients with Mild to Moderate Carotid Stenosis Predicts Recurrent Neurological Events. J Vasc Surg 2008;47:337-342.

44. Saam T, Cai J, Ma L, Cai YQ, Ferguson MS, Polissar NL et al. Comparison of Symptomatic and Asymptomatic Atherosclerotic Carotid Plaque Features with in Vivo Mr Imaging. Radiology 2006;240:464-472.

45. Mani V, Wong SK, Sawit ST, Calcagno C, Maceda C, Ramachandran S et al. Relationship between Particulate Matter Exposure and Atherogenic Profile in "Ground Zero" Workers as Shown by Dynamic Contrast Enhanced Mr Imaging Int J Cardiovasc Imaging 2013;29:827-833.

46. Tardif J-C. Heart Rate as a Treatable Cardiovascular Risk Factor. British Medical Bulletin 2009;90:7I-84.

47. Dominguez-Rodriguez A, Blanco-Palacios G, Abreu-Gonzalez P. Increased Heart Rate and Atherosclerosis: Potential Implications of Ivabradine Therapy. World journal of cardiology 20 I I;3: I0 I- I04.

48. Sun J, Canton G, Balu N, Hippe DS, Xu D, Liu J et al. Blood Pressure Is a Major Modifiable Risk Factor Implicated in Pathogenesis of Intraplaque Hemorrhage: An in Vivo Magnetic Resonance Imaging Study. Arterioscler Thromb Vasc Biol 2016; 10.1 161/atvbaha. I 15.307043.

49. Lobatto ME, Fayad ZA, Silvera S, Vucic E, Calcagno C, Mani V et al. Multimodal Clinical Imaging to Longitudinally Assess a Nanomedical Anti-Inflammatory Treatment in Experimental Atherosclerosis. Mol Pharm 20 10;7:20202029.

50. Vucic E, Dickson SD, Calcagno C, Rudd JH, Moshier E, Hayashi K et al. Pioglitazone Modulates Vascular Inflammation in Atherosclerotic Rabbits Noninvasive Assessment with Fdg-Pet-Ct and Dynamic ContrastEnhanced Mr Imaging. JACC Cardiovasc Imaging 20I I;4: I I00- I 109.

5I. Vucic E, Calcagno C, Dickson SD, Rudd JH, Hayashi K, Bucerius J et al. Regression of Inflammation in Atherosclerosis by the Lxr Agonist R21 1945: A Noninvasive Assessment and Comparison with Atorvastatin. JACC Cardiovasc Imaging 2012;5:819-828.

52. Dong L, Kerwin WS, Chen H, Chu B, Underhill HR, Neradilek MB et al. Carotid Artery Atherosclerosis: Effect of Intensive Lipid Therapy on the Vasa Vasorum—Evaluation by Using Dynamic Contrast-Enhanced Mr Imaging. Radiology 201 I;260:224-23I.

53. Nederveen AJ, Avril S, Speelman L. Mri Strain Imaging of the Carotid Artery: Present Limitations and Future Challenges. J Biomech 2014;47:824-833.

54. Wu T, Wang J, Song Y, Deng X, Li A, Wei J et al. Homologous Homologous Black-Bright-Blood and Flexible Interleaved Imaging Sequence (Hobbi) for Dynamic Contrast-Enhanced Mri of the Vessel Wall. Magn Reson Med 2015;73:1754-1763.

55. Calcagno C, Lobatto ME, Dyvorne H, Robson PM, Millon A, Senders ML et al. Three-Dimensional Dynamic Contrast-Enhanced Mri for the Accurate, Extensive Quantification of Microvascular Permeability in Atherosclerotic Plaques. NMR Biomed 2015;28:I304-I3|4.

56. de Rotte AA, Koning W, Truijman MT, den Hartog AG, Bovens SM, Vink A et al. Seven-Tesla Magnetic Resonance Imaging of Atherosclerotic Plaque in the Significantly Stenosed Carotid Artery: A Feasibility Study. Invest Radiol 2014;49:749-757. 
57. Koning W, de Rotte AA, Bluemink JJ, van der Velden TA, Luijten PR, Klomp DW et al. Mri of the Carotid Artery at 7 Tesla: Quantitative Comparison with 3 Tesla. J Magn Reson Imaging 2015;41:773-780.

58. Nguyen VL, Kooi ME, Backes WH, van Hoof RH, Saris AE, Wishaupt MC et al. Suitability of Pharmacokinetic Models for Dynamic Contrast-Enhanced Mri of Abdominal Aortic Aneurysm Vessel Wall: A Comparison. PLoS One 2013;8:e75173.

59. Nguyen VL, Backes WH, Kooi ME, Wishaupt MCJ, Hellenthal FAMVI, Bosboom EMH et al. Quantification of Abdominal Aortic Aneurysm Wall Enhancement with Dynamic Contrast-Enhanced Mri: Feasibility, Reproducibility, and Initial Experience. Journal of Magnetic Resonance Imaging 20 I4;39: I449-I 456. 


\section{SUMMARY}

Stroke is a large, health problem accounting for five million deaths and an additional five million disabled patients annually worldwide (I). In The Netherlands, stroke accounts for $21 \%$ of all cardiovascular deaths, with a total number of nearly 10.000 deaths in 2014 (2). Ischemic strokes, in which blood supply to (part of) the brain is (temporarily) impaired, are responsible for almost $90 \%$ of the strokes, with hemorrhagic strokes accounting for the remaining $10 \%$ (3). Of the ischemic strokes, 15-20\% are caused by atherosclerosis of the carotid artery (4).

In atherosclerosis, infiltration of macrophages and build up of fatty deposits and scar tissue leads to a local thickening of the vessel wall. Current guidelines for the treatment of patients with an atherosclerotic plaque in the carotid artery are based on the results of large randomized surgical trials performed in the early 90 s. These trials have shown that symptomatic patients with a stenosis of $70-99 \%$ that have experienced a recent cerebrovascular ischemic event benefit from carotid endartorectomy (CEA) with a number needed to treat (NNT) of 6. For symptomatic patients with a 50-69\% stenosis the NNT increases to 22, making CEA only marginally effective (5). During CEA, the vascular surgeon surgically removes the carotid artery plaque. Additionally, male patients with a carotid stenosis $\geq 50 \%$ who have experienced a recent ischemic event within two weeks are also eligible for CEA (6). Next to a CEA, symptomatic patients receive best medical treatment, consisting of anti-platelet therapy and cholesterol synthesis inhibitors (6).

Numerous histopathological studies performed on CEA specimen have shown that specific plaque characteristics are associated with an increased stroke incidence in symptomatic patients compared to asymptomatic patients (7-II). These "vulnerable plaques" are characterized by a large lipid-rich necrotic core (LRNC) with a thin fibrous cap, presence of inflammatory cells, ulcerations, and intraplaque hemorrhage (IPH) (I2), while the presence of fibrous tissue is considered a stabilizing feature (13). Next to these plaque features, increased microvasculature of the atherosclerotic plaque has been suggested to be a characteristic of plaque vulnerability (14). In physiological conditions, microvessels are already present in the outer layer of the vessel wall, the adventitia. However, in atherosclerosis, microvessels grow from the adventitia into the plaque tissue as a result of angiogenic stimuli. These newly formed microvessels generally have a low integrity of the endothelium (I5), providing a point of entry for inflammatory cells and erythrocytes. According to the fatigue hypothesis $(16,17)$, ruptured microvessels may also be the result of the biomechanical load on the atherosclerotic vessel wall due to exposure to the arterial pressure wave during each heart beat. These repetitive deformations of the atherosclerotic plaque may result in minor tissue damage, accumulating over time into larger tissue damage (crack propagation).

Non-invasive imaging techniques of the atherosclerotic vessel wall may provide opportunities for improved patient stratification. Firstly, vessel wall imaging enables more accurate determination of outward remodeling of the vessel wall compared to angiographic techniques, in which only the vessel lumen is displayed. Secondly, vessel wall imaging enables in vivo visualization of plaque characteristics. Interest in the potential benefit of patient stratification based on (a number of) these vulnerable plaque characteristics has greatly increased over the past de- 
cade, mainly owing to technical improvements of non-invasive imaging modalities. Moreover, since patients with a mild to moderate carotid stenosis rarely undergo CEA and the adventitial region of the vascular wall remains in situ after CEA, non-invasive imaging techniques enable an increased number of possibilities to study the role of vulnerable plaque characteristics over time. In particular patients with a mild to moderate stenosis may benefit from increased personalized treatment since for this patient population CEA is only marginally effective.

Non-invasive imaging and quantification of the plaque microvasculature can be performed using pharmacokinetic modeling of dynamic contrast-enhanced magnetic resonance imaging (DCE-MRI) (18-24). Recent studies showed a weak link between increased plaque microvasculature and plaque inflammation $(25,26)$ and the presence of intraplaque hemorrhage (27). However, it was shown that the correlation between plaque inflammation and microvasculature differs between symptomatic and asymptomatic plaques (26). Therefore, in vivo visualization of plaque microvasculature may not only provide further insight into pathological mechanisms involved in plaque development and destabilization, but may also provide additional information compared to other vulnerable plaque features.

The hypothesis of the present thesis is that non-invasive imaging of the plaque microvasculature with DCE-MRI can aid to gain further insight in the atherosclerotic process. To investigate this hypothesis, the following objectives are addressed in the present thesis:

- To further improve and validate DCE-MRI methodology

- To investigate the association between plaque microvasculature and important features of plaque vulnerability (plaque inflammation and presence of intraplaque hemorrhage)

- To explore whether plaque microvasculature is related to the type of cerebrovascular symptoms in patients with mild to moderate carotid stenosis

- To evaluate if DCE-MRI can be used as evaluation tool to investigate the effect of a heartrate reducing therapy on features of plaque vulnerability

In chapter 2, an overview of the current state of DCE-MRI in the evaluation of plaque microvasculature in clinical and preclinical settings is provided. First, principles and acquisition methods of DCE-MRI and methods for (semi-)quantitative analysis of DCE-MRI data are discussed. Secondly, an overview is given of publications in which DCE-MRI of plaque microvasculature is applied. The main findings are summarized on the following aspects I) the association of other plaque features with plaque microvasculature; 2) changes in plaque microvasculature over time; 3) comparison of different experimental animal groups and human subjects with a different cardiovascular risk profile; and 4) evaluation of the response to therapeutic interventions.

Accurate determination of the luminal contrast medium (CM) concentration (known as the vascular input function, VIF) is an essential requirement for quantitative analysis of DCE-MRI using pharmacokinetic modeling. In chapter 3, an alternative method for determination of the luminal CM concentration in DCE-MRI of the plaque microvasculature is introduced. This alternative method, based on the phase MRI signal, is compared to the currently commonly 
employed method based on the magnitude MRI signal. The results from this chapter show that the magnitude-based VIF is strongly influenced by local blood velocity, leading to an underestimation of the CM concentration in the vessel lumen. Analysis of the model parameters determined from both methods show a moderate to strong correlation between the two methods, although absolute values differ significantly. Based on the results of this chapter, it is advised to use a phase-based VIF for quantification of DCE-MRI data of carotid plaques.

The relationship between atherosclerotic plaque microvasculature and other features of plaque vulnerability has been studied in a number of DCE-MRI studies. However, these studies have focused on a single region of the vascular wall, i.e. either the entire vessel wall or the outer layer of the vessel wall only. Therefore, the interchangeability of these results is not clear. The aim of the study described in chapter 4 is I) to assess parameter agreement of $K^{\text {trans }}$ between the two vascular regions and 2) to study the correlation with microvessel density on histology. The results from this study showed similar moderately strong correlations with plaque microvessel density on histology, though a significantly higher median $\mathrm{K}^{\text {trans }}$ was found in the adventitia compared to the entire vessel wall. These results suggest that both vascular regions reflect plaque microvessel density, though care should be taken when comparing absolute values between studies that assessed different regions.

A previous DCE-MRI study in 27 patients has shown a positive association between adventitial $\mathrm{K}^{\text {trans }}$ and the presence of IPH within carotid plaque (27). In chapter 5 , the results of a study are presented with the aim to confirm the potential positive association between the plaque microvasculature and presence of IPH in a large cohort imaging study of $\mathrm{IOI}$ patients with carotid plaque. In this chapter, no difference in adventitial $\mathrm{K}^{\text {trans }}$ for patients with and without $\mathrm{IPH}$ is found, while a decreased vessel wall $\mathrm{K}^{\text {trans }}$ is found in patients with IPH. Therefore the positive association between plaque microvasculature and presence of IPH that was previously reported could not be confirmed in this chapter. The results of this chapter suggest that not only plaque microvasculature, but additional factors, such as a disrupted plaque surface, contribute to the development of IPH in carotid atherosclerosis. Several studies have already shown an important role for inflammation in the atherosclerotic process (28), which can be quantified using ${ }^{18} \mathrm{~F}-\mathrm{FDG}$ PET (29-3I). It is generally believed that activated macrophages lead to the formation of new microvessels. Therefore, plaque inflammation and plaque microvasculature may be linked to each other. In chapter 6 , the interchangeability of DCE-MRI and ${ }^{18} \mathrm{~F}-\mathrm{FDG}$ PET-CT is investigated in symptomatic patients with a mild carotid stenosis. In this chapter, a weak correlation coefficient $(\rho=0.30, p<0.05)$ between the two imaging modalities was found, showing that both imaging methods are related to each other. These results indicate that both modalities are not interchangeable and the relationship between plaque inflammation and (development of) plaque microvasculature may be time-dependent.

It is known from previous studies that patients with a recent ischemic stroke have a higher risk of recurrent stroke compared to (ocular) transient ischemic attack (TIA) patients. The purpose of the cross-sectional study described in chapter 7 is to explore the association between plaque microvasculature with type of recent cerebrovascular events in symptomatic 
patients with mild-to-moderate carotid stenosis. The results of this chapter show that the $75^{\text {th }}$ percentile adventitial $\mathrm{K}^{\text {trans }}$ is significantly associated with a recent ischemic stroke compared to (ocular) TIA in multivariate analysis, independent of clinical risk factors. These results indicate a positive association of leaky plaque microvasculature with a recent ischemic stroke compared to (ocular) TIA. Future prospective longitudinal studies are needed to further investigate whether $\mathrm{K}^{\text {trans }}$ may serve as an imaging marker to predict (the type of) future cerebrovascular events.

Epidemiologic studies have shown that patients with an elevated resting heart rate have a lower life expectancy (32-34), independent of other risk factors. An increased heart rate may result in minor repetitive tissue damages within the vessel wall. Accumulation over time may result in larger tissue damage (crack propagation), such as microvessel rupture, cap fissures, and ultimately, cap rupture, leading to further progression of atherosclerosis. Chapter 8 describes the results of a preclinical rabbit study in which the effect of a heart-rate reducing agent on features of atherosclerotic plaque vulnerability is investigated. To investigate the role of heart rate, atherosclerosis was induced in New Zealand White Rabbits by a combination of a cholesterol-enriched diet and balloon injury of the abdominal aorta and heart-rate reduction was achieved by administration of Ivabradine. Results of this study show that heart-rate reducing therapy is associated with a reduction in vulnerable plaque features. A decrease of macrophage content on histology was found. Analysis of DCE-MRI and the microvessel density on histological specimens together suggest a reduction of the plaque microvasculature leakiness, but not the microvessel density itself. Therefore, this chapter suggests that heart-rate reduction may be a potential target for plaque stabilization.

In chapter 9, the results of the present thesis are put into perspective of the current literature. Additionally, this chapter elaborates about possible future perspectives of DCE-MRI.

In conclusion, the results of this thesis show that non-invasive imaging of the plaque microvasculature with DCE-MRI is an important tool to gain further insight in the role of plaque microvasculature in the atherosclerotic process, contributing to the in depth understanding of the pathophysiology of atherosclerosis. 


\section{REFERENCES}

I. Mackay J, Mensah G. The Atlas of Heart Disease and Stroke - Global Burden of Stroke. 2004.

2. Van Dis I, Buddeke J, Vaartjes I, Visseren FLJ, Bots ML. Hart- En Vaatziekten in Nederland 2015, Cijfers over Heden, Verleden En Toekomst. Hartstichting 2015.

3. Mozaffarian D, Benjamin EJ, Go AS, Arnett DK, Blaha MJ, Cushman M et al. Heart Disease and Stroke Statistics-2016 Update: A Report from the American Heart Association. Circulation 2015;10.1161/ CIR.0000000000000350.

4. Chaturvedi S, Bruno A, Feasby T, Holloway R, Benavente O, Cohen SN et al. Carotid Endarterectomy-an Evidence-Based Review: Report of the Therapeutics and Technology Assessment Subcommittee of the American Academy of Neurology. Neurology 2005;65:794-80I.

5. Rothwell PM, Eliasziw M, Gutnikov SA, Fox AJ, Taylor DW, Mayberg MR et al. Analysis of Pooled Data from the Randomised Controlled Trials of Endarterectomy for Symptomatic Carotid Stenosis. Lancet 2003;36 I: I07-I I6.

6. Neurologie NVv. Richtlijn Diagnostiek, Behandeling En Zorg Voor Patiënten Met Een Beroerte. 2008.

7. Spagnoli LG, Mauriello A, Sangiorgi G, Fratoni S, Bonanno E, Schwartz RS et al. Extracranial Thrombotically Active Carotid Plaque as a Risk Factor for Ischemic Stroke. JAMA : the journal of the American Medical Association 2004;292: 1845-1852.

8. Redgrave JN, Lovett JK, Gallagher PJ, Rothwell PM. Histological Assessment of 526 Symptomatic Carotid Plaques in Relation to the Nature and Timing of Ischemic Symptoms: The Oxford Plaque Study. Circulation 2006; I 13:2320-2328.

9. Park AE, McCarthy WJ, Pearce WH, Matsumura JS, Yao JS. Carotid Plaque Morphology Correlates with Presenting Symptomatology. J Vasc Surg 1998;27:872-878; discussion 878-879.

10. Ballotta E, Da Giau G, Renon L. Carotid Plaque Gross Morphology and Clinical Presentation: A Prospective Study of 457 Carotid Artery Specimens. J Surg Res 2000;89:78-84.

II. Gao P, Chen ZQ, Bao YH, Jiao LQ, Ling F. Correlation between Carotid Intraplaque Hemorrhage and Clinical Symptoms: Systematic Review of Observational Studies. Stroke 2007;38:2382-2390.

12. Naghavi M, Libby P, Falk E, Casscells SW, Litovsky S, Rumberger J et al. From Vulnerable Plaque to Vulnerable Patient: A Call for New Definitions and Risk Assessment Strategies: Part I. Circulation 2003; 108: I 664-I 672.

13. Seeger JM, Barratt E, Lawson GA, Klingman N. The Relationship between Carotid Plaque Composition, Plaque Morphology, and Neurologic Symptoms. J Surg Res 1995;58:330-336.

14. Moreno PR, Purushothaman KR, Fuster V, Echeverri D, Truszczynska H, Sharma SK et al. Plaque Neovascularization Is Increased in Ruptured Atherosclerotic Lesions of Human Aorta: Implications for Plaque Vulnerability. Circulation 2004; I 10:2032-2038.

15. Sluimer JC, Kolodgie FD, Bijnens AP, Maxfield K, Pacheco E, Kutys B et al. Thin-Walled Microvessels in Human Coronary Atherosclerotic Plaques Show Incomplete Endothelial Junctions Relevance of Compromised Structural Integrity for Intraplaque Microvascular Leakage. J Am Coll Cardiol 2009;53:15 I7-I527.

16. Pei X, Wu B, Tang TY, Gillard JH, Li ZY. Fatigue Crack Growth under Pulsatile Pressure and Plaque Rupture. JACC Cardiovasc Imaging 2014;7:738-740.

17. Versluis A, Bank AJ, Douglas WH. Fatigue and Plaque Rupture in Myocardial Infarction. J Biomech 2006;39:339347.

18. Calcagno C, Mani V, Ramachandran S, Fayad ZA. Dynamic Contrast Enhanced (Dce) Magnetic Resonance Imaging (Mri) of Atherosclerotic Plaque Angiogenesis. Angiogenesis 2010;13:87-99.

19. Gaens ME, Backes WH, Rozel S, Lipperts M, Sanders SN, Jaspers K et al. Dynamic Contrast-Enhanced Mr Imaging of Carotid Atherosclerotic Plaque: Model Selection, Reproducibility, and Validation. Radiology 2013;266:27|-279.

20. Kerwin W. Quantitative Magnetic Resonance Imaging Analysis of Neovasculature Volume in Carotid Atherosclerotic Plaque. Circulation 2003; 107:85I-856.

21. Kerwin WS, O'Brien KD, Ferguson MS, Polissar N, Hatsukami TS, Yuan C. Inflammation in Carotid 
Atherosclerotic Plaque: A Dynamic Contrast-Enhanced Mr Imaging Study I. Radiology 2006;24I:459-468.

22. Kerwin WS, Oikawa M, Yuan C, Jarvik GP, Hatsukami TS. Mr Imaging of Adventitial Vasa Vasorum in Carotid Atherosclerosis. Magn Reson Med 2008;59:507-5I4.

23. Calcagno C, Cornily JC, Hyafil F, Rudd JHF, Briley-Saebo KC, Mani V et al. Detection of Neovessels in Atherosclerotic Plaques of Rabbits Using Dynamic Contrast Enhanced Mri and I8f-Fdg Pet. Arterioscler Thromb Vasc Biol 2008;28: I3 I I-1317.

24. Calcagno C, Vucic E, Mani V, Goldschlager G, Fayad ZA. Reproducibility of Black Blood Dynamic ContrastEnhanced Magnetic Resonance Imaging in Aortic Plaques of Atherosclerotic Rabbits. J Magn Reson Imaging 2010;32:191-198.

25. Calcagno C, Ramachandran S, Izquierdo-Garcia D, Mani V, Millon A, Rosenbaum D et al. The Complementary Roles of Dynamic Contrast-Enhanced Mri and I8f-Fluorodeoxyglucose Pet/Ct for Imaging of Carotid Atherosclerosis. European journal of nuclear medicine and molecular imaging 2013;40: I884- I893.

26. Wang J, Liu H, Sun J, Xue H, Xie L, Yu S et al. Varying Correlation between I8f-Fluorodeoxyglucose Positron Emission Tomography and Dynamic Contrast-Enhanced Mri in Carotid Atherosclerosis: Implications for Plaque Inflammation. Stroke 2014;45: I842- I845.

27. Sun J, Song Y, Chen H, Kerwin WS, Hippe DS, Dong L et al. Adventitial Perfusion and Intraplaque Hemorrhage: A Dynamic Contrast-Enhanced Mri Study in the Carotid Artery. Stroke 2013;44: I03 I- 1036.

28. Libby P. Inflammation in Atherosclerosis. Nature 2002;420:868-874.

29. Rudd JH, Warburton EA, Fryer TD, Jones HA, Clark JC, Antoun $\mathrm{N}$ et al. Imaging Atherosclerotic Plaque Inflammation with [18f]-Fluorodeoxyglucose Positron Emission Tomography. Circulation 2002; 105:2708-27II.

30. Rudd JH, Myers KS, Bansilal S, Machac J, Rafique A, Farkouh M et al. (I8)Fluorodeoxyglucose Positron Emission Tomography Imaging of Atherosclerotic Plaque Inflammation Is Highly Reproducible: Implications for Atherosclerosis Therapy Trials. J Am Coll Cardiol 2007;50:892-896.

31. Tawakol A, Migrino RQ, Hoffmann U, Abbara S, Houser S, Gewirtz H et al. Noninvasive in Vivo Measurement of Vascular Inflammation with F-18 Fluorodeoxyglucose Positron Emission Tomography. J Nucl Cardiol 2005; I2:294-30I.

32. Fox K, Borer JS, Camm AJ, Danchin N, Ferrari R, Lopez Sendon JL et al. Resting Heart Rate in Cardiovascular Disease. J Am Coll Cardiol 2007;50:823-830.

33. Lévy S, Guize L. [Heart Rate, a Major Prognostic Factor of Cardiovascular Risk]. Therapie 2006;61: I I 5-I I 9.

34. Palatini P. Heart Rate as an Independent Risk Factor for Cardiovascular Disease: Current Evidence and Basic Mechanisms. Drugs 2007;67 Suppl 2:3-I3. 



\section{RELEVANCE}

An estimated number of 240,000 people in the Netherlands are living with the consequences as a result of a stroke (I). In 2014, stroke accounted for $21 \%$ of all cardiovascular deaths, with a total number of nearly 10.000 deaths (2). Yearly, approximately 44,000 people are admitted to a hospital due to a stroke (I). Additionally, the medical costs involved in the diagnosis and treatment of stroke are substantial, accounting for $27.4 \%$ of the costs involved in cardiovascular care and $2.5 \%$ of the total medical costs in the Netherlands (3). It is expected that these numbers will increase over the coming decades, mainly because of the ageing population.

Hemorrhagic strokes account for approximately $10 \%$ of all strokes, while the remaining $90 \%$ are ischemic strokes (4). A considerable amount (15-20\%) of ischemic strokes is caused by rupture of an atherosclerotic plaque in the (internal) carotid artery (5). Currently, risk stratification of patients with a stenosis of the carotid artery is based on the degree of luminal stenosis and symptomatology of the patient. Based on this, it is determined whether a carotid endarterectomy (CEA) is advised. Results from randomized trials have shown that CEA is beneficial for symptomatic patients with a carotid stenosis of $70-99 \%$ with a number needed to treat (NNT) of 6 to prevent one stroke in five years. However, for symptomatic patients with a stenosis of $50-69 \%$ CEA is only marginally effective with a NNT of 22 (6).

Histological studies on CEA specimens have shown that specific characteristics of atherosclerotic plaques have an increased incidence in symptomatic patients compared to asymptomatic patients (7-II). Features already identified as important hallmarks of plaque vulnerability are a lipid-rich necrotic core (LRNC) with a thin/ruptured fibrous cap (TRFC), presence of inflammatory cells, ulcerations, and intraplaque hemorrhage (IPH) (I2). Additionally, it has been suggested that an increased microvasculature of the atherosclerotic plaque is an important marker of plaque vulnerability (I3).

Identification of these "vulnerable plaques" with non-invasive imaging of the atherosclerotic vessel wall may provide opportunities for improved patient stratification. In particular patients with a mild to moderate stenosis may benefit from increased personalized treatment since for this patient population CEA is only marginally effective. Over the past years, technical improvements of non-invasive imaging methods have enabled in vivo visualization of these vulnerable plaque characteristics.. Dynamic contrast-enhanced magnetic resonance imaging (DCE$\mathrm{MRI}$ ) can be used to study and quantify the plaque microvasculature non-invasively (I4).

The hypothesis of the present thesis was that non-invasive imaging of the plaque microvasculature with DCE-MRI may provide further insight in the atherosclerotic process. An increased insight in the role of the plaque microvasculature in the atherosclerotic process, may not only aid in identification of patients at increased risk, but may also provide novel targets for therapeutic interventions in the prevention and stabilization of atherosclerotic lesions. 


\section{TARGET GROUPS}

The results of this thesis are of interest for a broad range of professionals. First, it is of interest for scientists investigating the development of atherosclerotic plaques. Results from the present thesis and previous studies show that plaque microvasculature is an important determinant of atherosclerosis, since it is related to a number of processes related to plaque destabilization. Pharmacokinetic modelling of DCE-MRI data allows assessment of plaque microvasculature in a non-invasive manner. Therefore, the plaque microvasculature can be investigated in animal models and patients at multiple time points during the various stages of plaque development, even before the onset of clinical symptoms. This is of great advantage compared to histopathological studies, in which investigation of the plaque microvasculature can be performed only after euthanasia in animal studies and after CEA or at autopsy in patients. Non-invasive imaging of the microvasculature using DCE-MRI can contribute to the reduction of the amount of animals used in research.

Next to this, the results of this thesis are of interest for clinicians with a (research) interest in cerebrovascular disease. Currently, patients suffering from a recent ischemic cerebrovascular event undergo imaging of the carotid arteries for determination of the degree of carotid stenosis. Based on the results, patients may be referred to vascular surgery for CEA. However, there is increasing evidence, from the present thesis and other research, that patient stratification may be improved by assessment of the plaque components, including the plaque microvasculature. For further advancement of carotid plaque imaging, longitudinal studies are needed to investigate the predictive value of various plaque components, including plaque microvasculature for recurrent cerebrovascular events. For this purpose, the PARISk consortium in the Netherlands has performed longitudinal follow-up of patients that have suffered from a recent cerebrovascular event with patient follow-up of this trial finalized in December 2016, with results following in due time. The development of plaque microvasculature in relation to recurrent cerebrovascular events will be studied in a subgroup of these patients. After this prospective longitudinal study, it is important to investigate the potential value of dedicated imaging of carotid plaque microvasculature in a randomized surgery trial. Currently, imaging of various other carotid plaque components is already included in the recently started large randomized European Carotid Surgery Trial-2.

In addition to clinicians with a research interest in cerebrovascular diseases, the present thesis is also of general interest for clinicians. The results described in the present thesis may have impact on future guidelines for treatment of patients with carotid atherosclerotic plaque. Currently, patients with a mild to moderate stenosis are generally not referred to a vascular surgeon for surgical removal of the plaque, since randomized surgery trials performed in the early 90 s of the last century have shown that CEA is only marginally effective for them. These patients in particular may benefit from improved personalized treatment.

The present thesis is also of interest for companies within the medical imaging industry. The results described in this thesis show the current state of DCE-MRI of the atherosclerotic plaque microvasculature. The development of advanced MR imaging protocols and analysis methods for quantification of the plaque microvasculature is expected to further advance 
the clinical application of DCE-MR imaging. Additionally, companies developing (pharmacological) treatments or interventions targeted to the atherosclerotic plaque microvasculature may express interest in the results described in this thesis. DCE-MRI enables the investigation of the atherosclerotic plaque microvasculature non-invasively in animal models and patients. Compared to histopathological analysis, this enables evaluation of the treatment effect at multiple time points in the same subject, even before the onset of clinical symptoms. Thus, non-invasive imaging of the microvasculature using DCE-MRI can contribute to the reduction of the number of subjects, either animals or volunteers, that are required to study potential new treatments and their effect on the plaque microvasculature.

\section{ACTIVITIES / PRODUCTS}

In the present thesis, analysis of the DCE-MRI data is performed using custom built software in Matlab (Natick, Mathworks, Massachusetts, United States). In addition, drawing of the lumen and vessel wall contours is performed manually by an experienced observer using the Vesselmass software package (Leiden University Medical Center, Leiden, The Netherlands). This process may be suitable in a research environment, in which analysis is performed by experienced observers. However, this may be challenging in multicenter studies with analysis being performed by an increased number of observers. Therefore, development of a software package in which the lumen and vessel wall contour drawing and data analysis can be performed (semi-)automatically is of great interest. This software package is likely to be of added value for the potential success of DCE-MRI in a research or future clinical setting with less experienced observers, which is needed for implementation of the technique in a wider setting. On this topic, substantial progress has already been made based upon data from the PARISK consortium (15) and a spin-off company from the University of Washington (16). However, main focus of previous studies was the identification of the various plaque components on MR images while work on semi-automatic analysis tools for DCE-MR data has been limited. Extension of this with methods to perform (non-rigid) transformations of DCE-MRI data might be an important step in the development of DCE-MRI software packages. These transformations may be useful to correct for (small) patient movements during the MR acquisition, enabling improved (local) quantification of the plaque microvasculature. Additionally, integration of the developed methods into current software packages of the major MR vendors will be an important improvement in the availability of these methods.

\section{INNOVATION AND INSIGHTS}

Direct in vivo visualization of the plaque microvasculature is complicated due to the small size of the atherosclerotic plaque microvasculature (up to approximately $100 \mu \mathrm{m}$ in diameter). However, pharmacokinetic modelling of DCE-MRI allows voxelwise estimation of microvascular properties. In the present thesis several innovations are presented and applied to increase insights in the role of the plaque microvasculature in atherosclerotic plaques. 
Firstly, a novel, method for determination of the vascular input function (VIF) from phase MR images is described and applied for carotid DCE-MRI. The results from this thesis showed that VIFs derived from magnitude MR images were influenced by local blood flow velocity, leading to an underestimation of the contrast medium concentration in the vessel lumen. In turn, absolute values of determined pharmacokinetic parameters were influenced by the VIF, making direct cross-study comparisons of different studies difficult. Additionally, the results of the present thesis showed that the absolute values of the pharmacokinetic parameters determined may differ between different regions of the vascular wall. Therefore, the results of the present thesis clearly showed that the use of a standard imaging and data analysis protocol is essential, in particular for longitudinal studies of plaque microvasculature and for the future determination of risk thresholds.

One study described in the present thesis aimed to confirm the potential positive association between the plaque microvasculature and the presence of $\mathrm{PH}$, which has been suggested in previous research. However, the results of this chapter showed a decreased microvascular flow, density, or leakiness of the vessel wall in patients with IPH. Therefore, not only plaque microvasculature contributes to the development of $\mathrm{IPH}$ in carotid atherosclerosis. Based on our results, it is likely that additional factors, such as a disrupted plaque surface, also play an important role and should be investigated in future studies to fully understand the pathophysiology. Another important insight of the present thesis is that patients with a recent ischemic stroke showed an increased microvascular flow, density, or leakiness of the adventitia compared to patients with a recent transient ischemic attack (TIA) or ocular TIA (also known as amaurosis fugax). This association was independent of clinical risk factors. The potential association between plaque microvasculature and the type of recent cerebrovascular event has to be further confirmed in a prospective longitudinal study, however this finding may provide additional information on the value of DCE-MRI in the risk stratification of patients.

Finally, the present thesis demonstrated the ability of DCE-MRI as an evaluation tool for potential therapies in the treatment of atherosclerosis. The results showed that heart-rate reducing therapy was associated with a reduction in vulnerable plaque features. Therefore, the results of the present thesis added to current insights that plaque biomechanics and deformation may play an important role in the development of vulnerable plaque features. Therefore, heart rate reduction may be a potential target for plaque stabilization. To investigate the potential relationship between the plaque microvasculature and plaque biomechanics, including the underlying mechanisms, longitudinal studies incorporating both measurements should be performed in the future. 


\section{REFERENCES}

I. Koopman C, Van Dis I, Vaartjes I, Visseren FLJ, Bots ML. Hart- En Vaatziekten in Nederland 20 I4, Cijfers over Kwaliteit Van Leven, Ziekte En Sterfte. Hartstichting 2014.

2. Van Dis I, Buddeke J, Vaartjes I, Visseren FLJ, Bots ML. Hart- En Vaatziekten in Nederland 20I5, Cijfers over Heden, Verleden En Toekomst. Hartstichting 2015.

3. RIVM. Kosten Van Ziekten. $201 \mathrm{I}$.

4. Mozaffarian D, Benjamin EJ, Go AS, Arnett DK, Blaha MJ, Cushman M et al. Heart Disease and Stroke Statistics-2016 Update: A Report from the American Heart Association. Circulation 2015;10.116I/ CIR.0000000000000350.

5. Chaturvedi S, Bruno A, Feasby T, Holloway R, Benavente O, Cohen SN et al. Carotid Endarterectomy-an Evidence-Based Review: Report of the Therapeutics and Technology Assessment Subcommittee of the American Academy of Neurology. Neurology 2005;65:794-80I.

6. Rothwell PM, Eliasziw M, Gutnikov SA, Fox AJ, Taylor DW, Mayberg MR et al. Analysis of Pooled Data from the Randomised Controlled Trials of Endarterectomy for Symptomatic Carotid Stenosis. Lancet 2003;36I: $107-$ I 16.

7. Spagnoli LG, Mauriello A, Sangiorgi G, Fratoni S, Bonanno E, Schwartz RS et al. Extracranial Thrombotically Active Carotid Plaque as a Risk Factor for Ischemic Stroke. JAMA : the journal of the American Medical Association 2004;292: 1845-1852.

8. Redgrave JN, Lovett JK, Gallagher PJ, Rothwell PM. Histological Assessment of 526 Symptomatic Carotid Plaques in Relation to the Nature and Timing of Ischemic Symptoms: The Oxford Plaque Study. Circulation 2006; I 13:2320-2328.

9. Park AE, McCarthy WJ, Pearce WH, Matsumura JS, Yao JS. Carotid Plaque Morphology Correlates with Presenting Symptomatology. J Vasc Surg 1998;27:872-878; discussion 878-879.

10. Ballotta E, Da Giau G, Renon L. Carotid Plaque Gross Morphology and Clinical Presentation: A Prospective Study of 457 Carotid Artery Specimens. J Surg Res 2000;89:78-84.

II. Gao P, Chen ZQ, Bao YH, Jiao LQ, Ling F. Correlation between Carotid Intraplaque Hemorrhage and Clinical Symptoms: Systematic Review of Observational Studies. Stroke 2007;38:2382-2390.

12. Naghavi M, Libby P, Falk E, Casscells SW, Litovsky S, Rumberger J et al. From Vulnerable Plaque to Vulnerable Patient: A Call for New Definitions and Risk Assessment Strategies: Part I. Circulation 2003; 108:1664-I672.

13. Moreno PR, Purushothaman KR, Fuster V, Echeverri D, Truszczynska H, Sharma SK et al. Plaque Neovascularization Is Increased in Ruptured Atherosclerotic Lesions of Human Aorta: Implications for Plaque Vulnerability. Circulation 2004; I I0:2032-2038.

14. Calcagno C, Mani V, Ramachandran S, Fayad ZA. Dynamic Contrast Enhanced (Dce) Magnetic Resonance Imaging (Mri) of Atherosclerotic Plaque Angiogenesis. Angiogenesis 2010;13:87-99.

I5. van Engelen A. Multimodal Image Analysis for Carotid Artery Plaque Characterization. Rotterdam: Erasmus University Rotterdam; 2014.

16. Kerwin W, Xu D, Liu F, Saam T, Underhill H, Takaya N et al. Magnetic Resonance Imaging of Carotid Atherosclerosis: Plaque

17. Kerwin W, Xu D, Liu F, Saam T, Underhill H, Takaya N et al. Magnetic Resonance Imaging of Carotid Atherosclerosis: Plaque Analysis. Top Magn Reson Imaging 2007; 18:37I-378. 
Een promotie wordt vaak gezien als een individuele prestatie, net zoals wielrennen door veel mensen als een individuele sport gezien wordt. Beiden zijn echter niet correct! Een promotie, en mijn promotie is daar geen uitzondering op, is te vergelijken met het rijden van een grote meerdaagse (wieler)koers als kopman. Het behalen van de overwinning in deze wielerkoers, de promotieplechtigheid, zou niet mogelijk zijn geweest zonder de (directe of indirecte) medewerking en/of steun van vele betrokkenen.

Als eerste is daar ploegleiding in de vorm van de sportief directeur en ploegleiders. Zij hebben een belangrijke bijdrage in het opstellen van het tactische plan om de eindzege te behalen. Als eerste wordt gekeken wat de beste voorbereiding is. Welke wedstrijden worden gereden? Wel of geen trainingsstage op hoogte? Alles om ervoor te zorgen dat de kopman uiteindelijk zo optimaal mogelijk aan de start kan verschijnen. Dan is daarnaast de ploeg van collega renners die zullen deelnemen aan de wielerkoers. Welke andere renners zijn er beschikbaar om de kopman te ondersteunen tijdens de koers? Wat zijn hun kwaliteiten en hoe kunnen zij hiermee de kopman van dienst zijn? In de voorbereiding kan ook al uitgebreid aandacht besteed worden aan verkenning van het parcours. Wat is het parcours? Welke cols moeten er beklommen worden? Wat zijn de scherprechters in het parcours? Dit zijn allemaal belangrijke aspecten in de voorbereiding waar de ploegleiding een aandeel in heeft.Maar ook als de koers eenmaal begonnen is, is er een belangrijke rol weggelegd voor de ploegleiding. Als eerste kunnen zij tijdens de etappe, vanuit de ploegleiderswagen, achter het peloton, het overzicht op de gehele wedstrijd bewaren en via de communicatie de renners alle benodigde informatie geven over de kopgroep en hun voorsprong op het peloton. Tegelijktertijd kunnen de renners via de oortjes communiceren met de ploegleiding en deze op de hoogte brengen van onder meer mechanische problemen zodat de ploegleiding hier snel op kan reageren. Ten tweede heeft de ploegleiding tijdens een meerdaagse etappekoers een belangrijke rol bij de evaluatie na iedere etappe. Wat is er gebeurd in de afgelopen etappe? Welke zaken gingen er goed of kunnen de volgende keer juist anders? Wat is op het moment de tussenstand in het algemeen klassement? Is deze tussenstand nog volgens de verwachting? Welke etappes staan er voor de volgende dag(en) gepland? Zijn er veranderingen waardoor het opgestelde plan aangepast moet worden? Welke mogelijkheden zijn er nog om de eindoverwinning te behalen? Allemaal vragen die tijdens de (tussentijdse) evaluaties tussen de kopman en de ploegleiding aan de orde (kunnen) komen. Tijdens dit proces kan het weleens voorkomen dat er onderling strubbelingen zijn. De kopman wil tijdens de voorbereiding van een grote ronde deelnemen aan een wielerklassieker of (kleinere) wedstrijd die door zijn "achtertuin" gaat, terwijl de ploegleiding een hoogtestage wil inplannen in deze periode. Of er zijn, tot ongenoegen van de ploegleiding, geen renners mee zijn in de ontsnapping. Maar daarnaast zijn er ook veel positieve momenten zoals wanneer de kopman boven zichzelf uitstijgt in de afsluitende tijdrit en zo de overwinning in het algemeen klassement binnenhaalt.

Om succesvol te zijn in de koers te zijn is niet alleen de ploegleiding van belang, maar ook de ploeggenoten zijn onmisbaar tijdens de koers. leder van de ploeggenoten heeft zijn specifieke kwaliteiten welke in verschillende situaties optimaal tot hun recht komen. Omdat er een maximum aantal ploeggenoten aan een wedstrijd kunnen deelnemen moet er voor voor 
iedere wedstrijd een selectie van ploeggenotem gemaakt worden. Zoals gezegd is deze selectie toegespitst op de wedstrijd en de individuele kwaliteiten van de renners. Dit betekent ook dat een aantal renners maar aan een beperkt aantal wedstrijden zullen deelnemen terwijl er ook een select gezelschap van ploeggenoten is dat tijdens bijna alle koersen aan de zijde van de kopman staan. Deze ploeggenoten (ook wel meesterknechten genoemd) hebben dan ook een belangrijk aandeel in de eindoverwinning. Zonder hun uitzonderlijke inspanningen en steun op cruciale momenten tijdens de verschillende wedstrijd zou een succesvol resultaat vrijwel ondenkbaar zijn.

Achter de schermen is een wielerploeg nog veel meer dan alleen de ploegleiding en wielrenners. Zonder ondersteunend personeel kan zelfs de beste ploeg niet optimaal presteren. Als eerste zijn daar de soigneurs en mechaniciens. Na afloop van de etappe of wedstrijd worden de coureurs door de soigneurs onder handen genomen zodat zij optimaal kunnen uitrusten en hersteld aan de start van de volgende etappe staan. Tegelijkertijd zorgen de mechaniciens ervoor dat de fiets voor de volgende etappe weer tip top in orde is. Terwijl de werkzaamheden van de mechaniciens en soigneurs direct aan het wielrennen gerelateerd is, is er nog meer ondersteunend personeel dat belangrijk is voor het reilen en zeilen van een wielerploeg. Ondersteunend personeel zoals administratieve medewerkers die ervoor zorgen dat alles goed georganiseerd is. Door hen worden allerlei hand- en spandiensten rondom de ploeg geregeld, zoals het regelen van een taxi om de renner bij het vliegveld op te halen tot het verzorgen van een persconferentie.

Het is natuurlijk wel belangrijk dat een overwinning op een eerlijke manier behaald wordt. Gelukkig zijn er verschillende overkoepelende organisaties die, als externe partij, op handhaving van de regels toezien. Dit gebeurt zowel voor, tijdens, als na de wedstrijd. Voor de wedstrijd, tijdens de voorbereiding, voeren dopingcontroleurs (onverwachte) out-of-competition testen uit. Tijdens de koers is er de wedstrijdjury die op een eerlijke wedstrijd toezien. Zo zullen zij bijvoorbeeld ingrijpen wanneer een wielrenner na een lekke band door (te veel) hulp van de ploegwagen terug kan keren. En na afloop van de wedstrijd is er natuurlijk de anti-doping controle als laatste controle dat alles volgens de regelementen is verlopen.

Naast een goede professionele begeleiding is een stabiele, privé omgeving van groot belang. Het begrip en de steun van familie en vrienden is even belangrijk en moet niet worden onderschat. Regelmatig moeten er immers concessies worden gedaan. Dit kan varieren van kleine, relatief onschuldige dingen (bijvoorbeeld het afwijzen van een alcoholische versnapering op een feestje) tot grote opofferingen zoals het, zonder familie en vrienden, verhuizen naar Spanje of Italië voor een betere trainingsklimaat. Tegelijkertijd biedt het wielrennen ook veel gezellige momenten en unieke mogelijkheden. Het bezoeken van de mooiste plaatsen van de wereld om je werk uit te voeren, van de Tour Down Under tot de Tour of California of de Grand Prix Cycliste de Quebec et Montreal, iets waar veel mensen alleen maar van kunnen dromen. 
Via deze weg wil ik graag al mijn sportief directeurs, ploegleiders, ploeggenoten, soigneuers, mechaniciens, ondersteuners, trainingspartners, vrienden en familie bedanken voor hun vertrouwen, geduld en steun tijdens de wielerkoers die mijn promotie is geweest. Zonder dit vertrouwen, geduld en steun had ik deze etappekoers met meerdere koninginnenritten niet kunnen volbrengen. Jullie hebben ervoor gezorgd dat ik deze Cinglé heb kunnen volbrengen. Jeire, ik ben er helemaal trots op dat we er samen een 'bicinglette' van hebben gemaakt.

Als laatste wil ik graag afsluiten met een uitspraak van de grootste wielrenner aller tijden, 'De Kannibaal' Eddy Merckx: "Ride as much or as little, or as long or as short as you feel. But ride." 


\section{Credits}

Management / Ploegleiding

Sportief directeurs Prof.dr. J.E. Wildberger, Prof.dr. S. Heeneman

Ploegleiding Dr. M.E. Kooi

Wedstrijdjury

Voorzitter. Prof. dr. F.M. Mottaghy Juryleden Prof.dr. E. Biessen, Dr. J.W.H.C. Daemen Prof.dr. P. Hofman, Prof.dr. T. Leiner

\section{Coureurs}

Dr. M.E.P. Philippens, Prof.dr. M.J. Post

Meesterknechten Evelien, Floris, Martine, Stefan Ploeggenoten .Arnold, Jack, Jan, Jan-Willem Jérôme, Judith, Julie, Mat Narander, Nicky, Patty, Paul Rob, Robert, Robert, Tobien Walter, Werner

\section{Ondersteunend personeel}

Mechanciens / soigneurs Anique, Clairy, Jos, Joyce Petra, Sanne

Administratie Christianne, Elfie, Monique, Monique

Privé

Vrienden. Anne, Anneloes, Bart, Elien Ellen K, Ellen N, Esther \& Jelle Germaine, Joel, Joke \& Marcel Mark, Melanie, Rob, Stefan Stijn \& Leola, Yvonne

Trainingsgroep Carmen, Esther, Fred, Frits Inge, Jan, Jan, Lex Luc, Marc, Mechiel, Michel Pierre, Ralph, Roger, Ron

Familie Toine, Karin \& Krista Maarten \& Inge Partner ..Jeire 

Raf van Hoof was born on the 26th of October 1988 in Grubbenvorst, The Netherlands. From 2000 until 2006 he attended secondary education at the Blariacumcollege in Blerick where he obtained his high school diploma (gymnasium).

In September 2006 he started his academic education at Eindhoven University of Technology where he obtained his Master of Science degree in Biomedical Engineering in December 20II. From December 20II onwards, he worked as a PhD candidate at the department of Radiology (CARIM School for Cardiovascular Diseases, Maastricht University) under the supervision of Prof.dr. Joachim E. Wildberger, Prof.dr. Sylvia Heeneman, and Dr. M. Eline Kooi. During his $\mathrm{PhD}$ project he aimed to investigate the role of the microvasculature within atherosclerotic lesions using Dynamic Contrast-Enhanced MRI. This research was performed within the framework of the Center for Translational Medicine (www.ctmm.nl), project PARISK, and was supported by the Dutch Heart Foundation. 


\section{Scientific Publications}

RHM van Hoof, S Heeneman, JE Wildberger, ME Kooi. Dynamic Contrast-Enhanced MRI to Study Atherosclerotic Plaque Microvasculature. Current Athersoclerosis Reports. 20 I6; I8(33)

RHM van Hoof, E Hermeling, MTB Truijman, RJ van Oostenbrugge, JWH Daemen, RJ van der Geest, NP van Orshoven, AH Schreuder, WH Backes, MJAP Daemen, JE Wildberger, ME Kooi. Phase-Based Vascular Input Function: Improved Quantitative DCE-MRI of Atherosclerotic Plaques. Medical Physics. 2015;42(8) 4619-4628

RHM van Hoof, SA Vöö, JC Sluimer, NJA Wijnen, E Hermeling, FHBM Schreuder, MTB Truijman, JPM Cleutjens, MJAP Daemen, JWH Daemen, RJ van Oostenbrugge, WH Mess, JE Wildberger, S Heeneman, ME Kooi. Vessel Wall and Adventitial DCE-MRI Parameters Demonstrate Similar Correlations with Carotid Plaque Microvasculature on Histology. Journal of Magnetic Resonance Imaging. 2017;doi 10.1002/jmri.25648

RHM van Hoof, FHBM Schreuder, MTB Truijman, NJA Wijnen, S Heeneman, PJ Nederkoorn, WH Mess, RJ van Oostenbrugge, JWH Daemen, MJAP Daemen, JE Wildberger, ME Kooi. Microvasculature and Intraplaque Hemorrhage in Atherosclerotic Carotid Lesions: an MR Imaging Study. Submitted

MTB Truijman, RM Kwee, RHM van Hoof, E Hermeling, RJ van Oostenbrugge, WH Mess, WH Backes, MJAP Daemen, J Bucerius, JE Wildberger, ME Kooi. Combined I 8F-FDG PET-CT and DCE-MRI to Assess Inflammation and Microvascularization in Atherosclerotic Plaques. Stroke. 2013 Dec;44(I2):3568-3570

RHM van Hoof, FHBM Schreuder, P Nelemans, MTB Truijman, NP van Orshoven, AH Schreuder, WH Mess, S Heeneman, RJ van Oostenbrugge, JE Wildberger, ME Kooi. Increased Plaque Microvasculature is Associated with Stroke Subtype in Symptomatic Patients with Carotid Plaque: an MRI Study. Submitted

RHM van Hoof, E Hermeling, JC Sluimer, J Salzmann, APG Hoeks, J Roussel, MJAP Daemen, H Struijker-Boudier, JE Wildberger, S Heeneman, ME Kooi. Heart Rate Lowering Treatment Leads to a Reduction in Vulnerable Plaque Features in Atherosclerotic Rabbits. Submitted

VL Nguyen, ME Kooi, WH Backes, RHM van Hoof, AECM Saris, MC Wishaput, FA Hellenthal, RJ van der Geest, AG Kessels, GW Schurink, T Leiner. Suitability of Pharmacokinetic Models for Dynamic Contrast-Enhanced MRI of Abdominal Aortic Aneurysm Vessel Wall. PLoS One. 2013 Oct 2;8(10):e75713

M Wolters, RHM van Hoof, A Wagenaar, K Douma, MAMJ van Zandvoort, TH Hackeng, MJ Post, WH Backes, ME Kooi. MRI Artifacts in the Ferric Chloride Thrombus Animal Model: an Alternative Solution: Preventing MRI Artifacts after Thrombus Induction with a non-ferromagnetic Lewis Acid. J Thromb Haemost. 2013 Sep; I I (9): I 766- 1769 


\section{Oral Conference Presentations}

RHM van Hoof', E Hermeling', J Salzmann, JC Sluimer, S Heeneman, APG Hoeks, J Roussel, H Struijker-Boudier, JE Wildberger, ME Kooi. The Effect of Ivabradine on Plaque Size, Biomechanics, and Microvasculature in Atherosclerotic Rabbits Measured using MR, Ultrasound Imaging, and Histology. ESMRMB 2015 - 32nd Annual Scientific Meeting, Edinburgh, United Kingdom (October I-3, 20I5)

E Hermeling', RHM van Hoof', J Salzmann, JC Sluimer, S Heeneman, APG Hoeks, J Roussel, H Struijker-Boudier, JE Wildberger, ME Kooi. The Effect of Ivabradine on Plaque Size, Biomechanics, and Microvasculature in Atherosclerotic Rabbits. ARTERY I4, Maastricht, The Netherlands (October 9-1।, 2014)

RHM van Hoof, E Hermeling, NJA Wijnen, FHBM Schreuder, MTB Truijman, SA Voo, JPM Cleutjens, JC Sluimer, S Heeneman, RJ van Oostenbrugge, JWH Daemen, MJAP Daemen, JE Wildberger, ME Kooi. Comparison of Various DCE-MRI Model Parameters in Carotid Plaques: Influence of Analysis Method and Correlation with Histology. 26th International Conference MRA Working Group, Rome, Italy (September 16-19, 2014)

RHM van Hoof', E Hermeling', J Salzmann, JC Sluimer, S Heeneman, APG Hoeks, J Roussel, $\mathrm{H}$ Struijker-Boudier, JE Wildberger, ME Kooi. MR and Ultrasound Imaging of the Effect of Ivabradine on Plaque Size, Biomechanics, and Microvasculature in Atherosclerotic Rabbits. 26th International Conference MRA Working Group, Rome, Italy (September I6-19, 2014) I

RHM van Hoof, MTB Truijman, FHBM Schreuder, E Hermeling, SA Voo, RJ van der Geest, WH Backes, RJ van Oostenbrugge, JWH Daemen, MJAP Daemen, JE Wildberger, ME Kooi. Comparison of Various Dynamic Contrast-Enhanced MRI Model Parameters in Carotid Plaques. 6th ISMRM Benelux Chapter Meeting 20I4, Maastricht, The Netherlands (January 20, 2014)

RHM van Hoof, MTB Truijman, E Hermeling, RJ van Oostenbrugge, RJ van der Geest, MJAP Daemen, JE Wildberger, WH Backes, ME Kooi. Influence of magnitude- versus phase-based vascular input function on Dynamic Contrast-Enhanced MRI model parameters of carotid plaques. ESMRMB 2013 - 30rd Annual Scientific Meeting, Toulouse, France (October 3-5, 20I3)

RHM van Hoof, MTB Truijman, E Hermeling, RJ van Oostenbrugge, RJ van der Geest, AH Schreuder, AGGC Korten, NP van Orshoven, B Meens, MJAP. Daemen, JE Wildberger, WH Backes, ME Kooi. Determination of the Vascular Input Function using Magnitude or Phase-Based MRI: Influence on Dynamic Contrast-Enhanced MRI Model Parameters in Carotid Plaques. ISMRM 2 I st Annual Meeting and Exhibition, Salt Lake City, Utah, USA (April 20-26, 20I3)

\footnotetext{
I Shared first author
} 
RHM van Hoof, MTB Truijman, E Hermeling, RJ van Oostenbrugge, RJ van der Geest, MJAP Daemen, JE Wildberger, WH Backes, ME Kooi. Determination of the Vascular Input Function using Magnitude or Phase-Based MRI: Influence on Dynamic Contrast-Enhanced MRI Model Parameters in Carotid Plaques. European Congress of Radiology, Vienna, Austria (March 7-।I, 2013)

RHM van Hoof, MTB Truijman, E Hermeling, RJ van Oostenbrugge, RJ van der Geest, AH Schreuder, AGGC Korten, NP van Orshoven, B Meens, MJAP Daemen, JE Wildberger, WH Backes, M.E. Kooi. Determination of the Vascular Input Function using Magnitude or Phase-Based MRI: Influence on Dynamic Contrast-Enhanced MRI Model Parameters in Carotid Plaques. 5th ISMRM Benelux Chapter Meeting 2013, Rotterdam, The Netherlands (January $14,2013)$

\section{Conference Poster Presentations}

RHM van Hoof, E Hermeling, NJA Wijnen, FHBM Schreuder, MTB Truijman, SA Voo, JPM Cleutjens, JC Sluimer, S Heeneman, RJ van Oostenbrugge, JWH Daemen, MJAP Daemen, JE Wildberger, ME Kooi. Dynamic Contrast-Enhanced MRI Model Parameters from Different Regions Within the Vascular Wall of Carotid Plaques: Comparison with Histology. ISMRM 23rd Annual Meeting and Exhibition, Toronto, Ontario. Canada (May 30-June 5, 20I5)

RHM van Hoof, E Hermeling, J Salzmann, JC Sluimer, S Heeneman, APG Hoeks, HAJ Struijker-Boudier, J Roussel, JE Wildberger, ME Kooi. The Effect of Ivabradine on Plaque Size, Biomechanics, and Microvasculature in Atherosclerotic Rabbits measured using MR and Ultrasound Imaging. ISMRM 23rd Annual Meeting and Exhibition, Toronto, Ontario. Canada (May 30-June 5, 2015)

RHM van Hoof, E Hermeling, J Salzmann, JC Sluimer, S Heeneman, APG Hoeks, HAJ Struijker-Boudier, J Roussel, JE Wildberger, ME Kooi. Effect of Ivabradine on Plaque Size, Biomechanics, and Microvasculature in Atherosclerotic Rabbits Measured using MR and Ultrasound. 7th ISMRM Benelux Chapter Meeting 20I5, Ghent, Belgium (January I6, 20I5)

RHM van Hoof, E Hermeling, NJA Wijnen, FHBM Schreuder, MTB Truijman, SA Voo, JPM Cleutjens, JC Sluimer, S Heeneman, RJ van Oostenbrugge, JWH Daemen I, MJAP Daemen, JE Wildberger, ME Kooi. Carotid Plaque Microvasculature Assessed using DCE-MRI: Comparing Different Regions of the Vascular Wall. ARTERY I4, Maastricht, The Netherlands (October 9- II, 2014) 


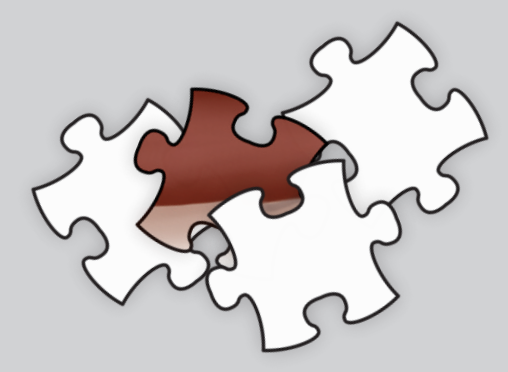

20

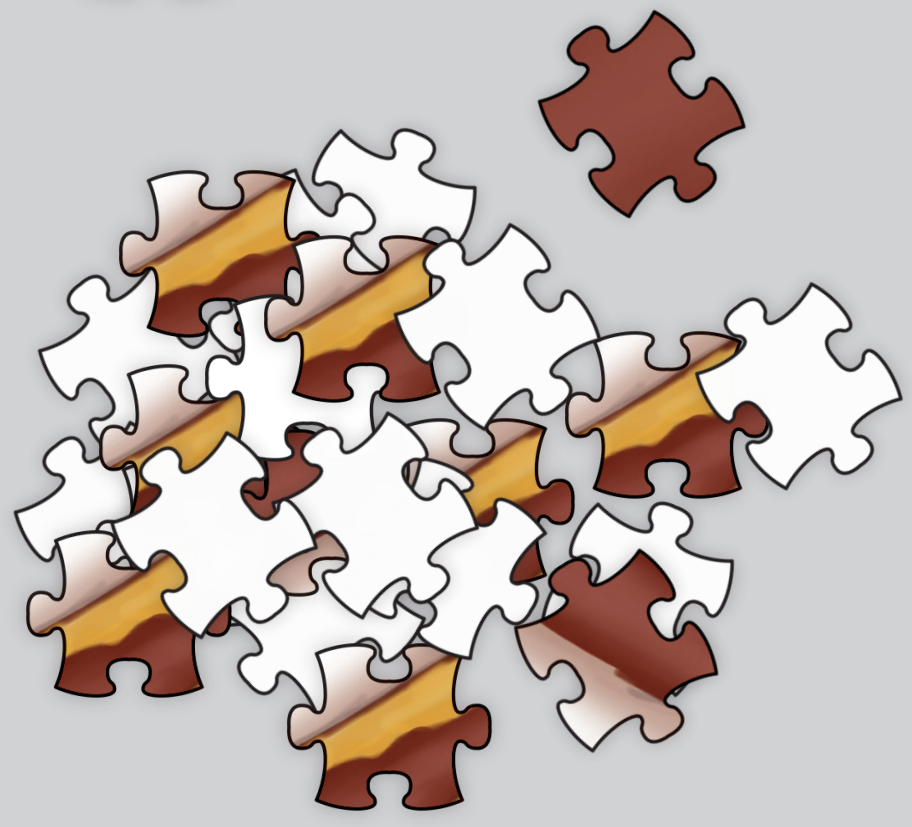

